\title{
Building Congested Ketones: Substituted Hantzsch Ester and Nitrile as Alkylation Reagents in Photoredox Catalysis
}

\author{
Wenxin Chen ${ }^{\mathrm{a}}$, Zheng Liu ${ }^{\mathrm{a}}$, Jiaqi Tian ${ }^{\mathrm{b}}$, Jin $\mathrm{Li}^{\mathrm{a}}$, Jing $\mathrm{Ma}^{\mathrm{b}}$, Xu Cheng ${ }^{\mathrm{a}^{*}}$, Guigen $\mathrm{Li}^{\mathrm{a}, \mathrm{c}}$ \\ a Institute of Chemistry and Biomedical Sciences, School of Chemistry and Chemical Engineering, Nanjing \\ University, Nanjing, China \\ b Key Laboratory of Mesoscopic Chemistry of MOE, Collaborative Innovation Center of Chemistry for Life \\ Sciences, School of Chemistry and Chemical Engineering, Nanjing University, Nanjing, China \\ c Department of Chemistry and Biochemistry, Texas Tech University, Lubbock, Texas, United States
}


Content

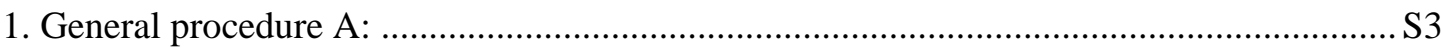

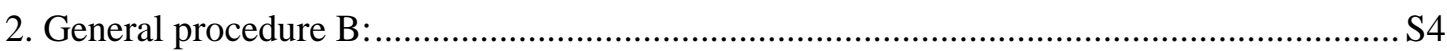

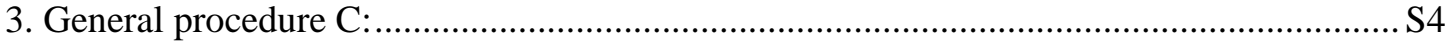

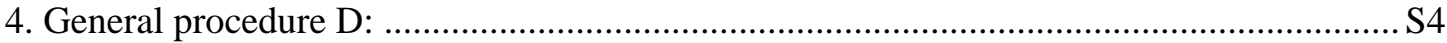

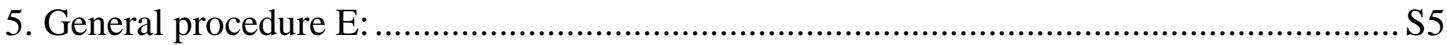

6. General procedure F for visible-light induced photoredox alkylation reaction with Hantzsch ester S6

7. General procedure $\mathrm{G}$ for visible-light induced photoredox alkylation reaction with Hantzsch nitrile S6

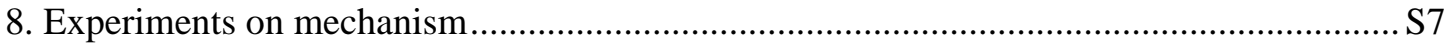

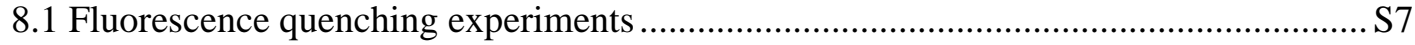

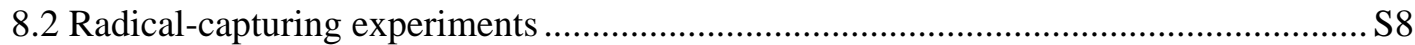

8.3 Spectroscopic study of Ir species and experiments with transition metal additives .... S12

8.4 Isolation of dimerized benzyl radical from standard reaction ................................... S15

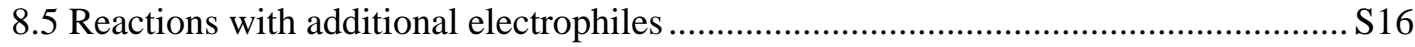

8.6 Reaction to investigate if a radical chain pathway was involved ................................S16

8.7 Reactions employing analougues of 1a with less hinderance ......................................S17

8.8 Radical clock experiment and reactions with hydride donors ..................................S18

8.9 Reaction of Hantzsch ester and bromobenzene in the presence of transition metal catalysts S20

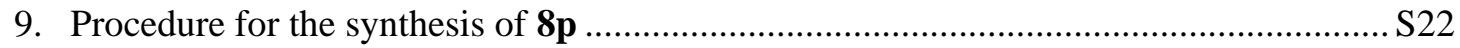

10. Procedure for synthesis of $\mathbf{9}$ and cultivation of single crystals................................... S22

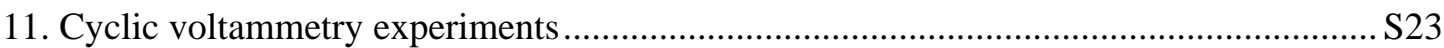

12. Procedure for syntheses of 2,6-dimethylpyridine-3,5-dicarboxylates and their electric potential S26

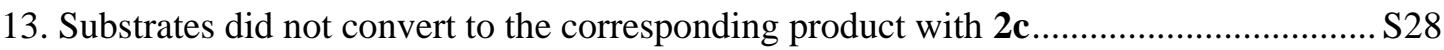

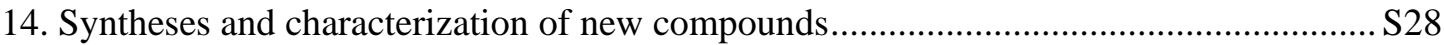

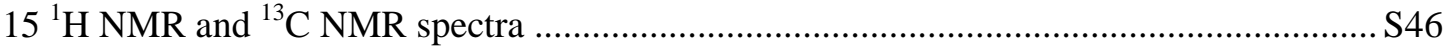

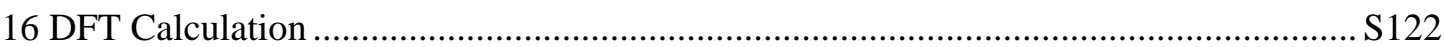




\section{General information}

All reactions were carried with standard procedures using Schlenk techniques unless specified otherwise. Commercially available reagents from J\&K Chemical, HEOWNS, Energy Chemical and Aldrich were used as received. The solvents were distilled from the appropriate drying reagents.

Thin layer chromatography (TLC) was performed using glass-backed silica gel plates Flash chromatography utilized 300-400 mesh silica gel from Qingdao Haiyang Chemical Co., Ltd. Products were visualized with UV light at $254 \mathrm{~nm}$.

${ }^{1} \mathrm{H}$ NMR spectra were recorded on Bruker Avance III 400 (400 MHz). Chemical shifts were reported in ppm downfield from tetramethylsilane with the solvent resonance as the internal standard $\left(\mathrm{CDCl}_{3}, \delta=7.26\right)$. Spectra were reported as follow: chemical shift $(\delta \mathrm{ppm})$, multiplicity (s = singlet, $\mathrm{d}$ $=$ doublet, $\mathrm{t}=$ triplet, $\mathrm{q}=$ quartet, $\mathrm{m}=$ multiplet $)$, coupling constants $(\mathrm{Hz})$ and integration. ${ }^{13} \mathrm{C} \mathrm{NMR}$ spectra were collected on Bruker Avance III 400 (100 MHz) with complete proton decoupling. Chemical shifts are reported in ppm downfield from the tetramethylsilane with the solvent resonance as internal standard $\left(\mathrm{CDCl}_{3}, \delta=77.0\right)$. IR spectra were recorded on ThermoFisher Nicolet iS5. Fluorescence quenching experiments were performed on HITACHI F-7000FL 5J1-0004. Mass spectra were recorded on Agilent system equipped with an ESI or APCI source, or Waters Micromass GCT Premier EI source.

\section{General procedure A:}

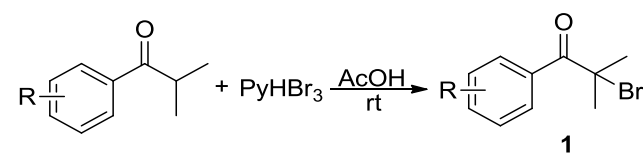

A mixture of substituted isobutyrophenones (1.0 mmol), $\mathrm{PyHBr}_{3}(320 \mathrm{mg}$ or $960 \mathrm{mg}, 1.0 \mathrm{mmol}$ or 3.0 $\mathrm{mmol})$ and $\mathrm{AcOH}(3.0 \mathrm{~mL})$ was stirred at room temperature for 10 hours. After the reaction was complete, the mixture was diluted with $\mathrm{H}_{2} \mathrm{O}$. The resulting mixture was extracted with dichloromethane for 3 times. The combined organic layers were washed with saturated aqueous $\mathrm{NaHCO}_{3}$ solution and brine in turn, dried $\left(\mathrm{MgSO}_{4}\right)$, and filtered. The filtrate was concentrated under reduced pressure. The residue was used directly or purified by chromatography on silica gel if necessary. 1a, ${ }^{[1]} 1 \mathbf{b},{ }^{[2]} 1 \mathbf{c},{ }^{[2]} 1 \mathrm{~d},{ }^{[2]} 1 \mathbf{g},{ }^{[3]} 1 \mathbf{k},{ }^{[1]} 1 \mathbf{1},{ }^{[4]} 1 \mathbf{c o},{ }^{[5]} 1 \mathbf{s},{ }^{[6]} 1 \mathbf{t}^{[7]}$ and $1 \mathrm{x}^{[8]}$ are known compounds.

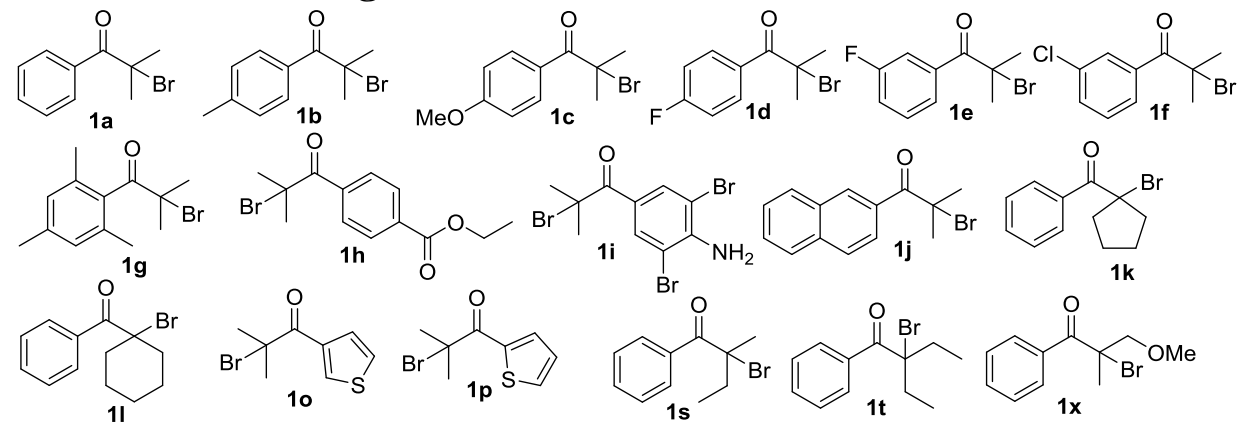

Figure S1 Synthesized $\alpha$-bromo ketones with procedure A. 


\section{General procedure B:}

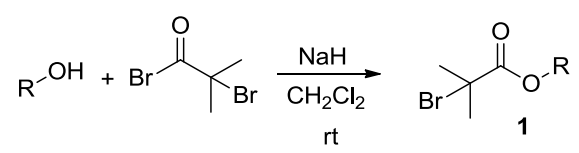

A flask was charged with phenol or alcohol $(1.0 \mathrm{mmol}), \mathrm{NaH}(40 \mathrm{mg}, 1.0 \mathrm{mmol}$, 60\% dispersion on mineral oil $)$ and $\mathrm{CH}_{2} \mathrm{Cl}_{2}(2.0 \mathrm{~mL})$. The mixture was stirred for 5 minutes at room temperature and then 2-bromo-2-methylpropionyl bromide $(230 \mathrm{mg}, 1.0 \mathrm{mmol})$ was added slowly. The reaction was monitored by TLC until complete conversion of phenol or alcohol was achieved. Then the reaction was quenched with water and the resulting mixture was extracted with $\mathrm{CH}_{2} \mathrm{Cl}_{2}$ for 3 times. The combined organic layers were washed brine, dried $\left(\mathrm{MgSO}_{4}\right)$, and filtered. The filtrate was concentrated under reduced pressure The residue was used directly without further purification. 1q is known compound. [9]
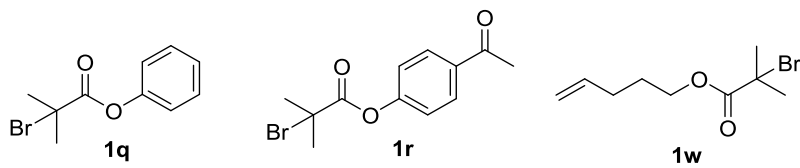

Figure S2 Synthesized $\alpha$-bromo esters with procedure B

\section{General procedure C:}

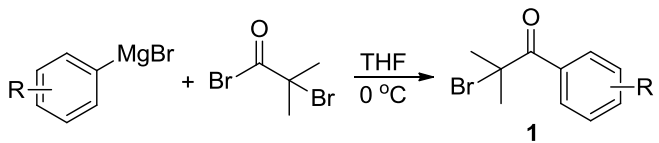

A flask charged with 2-bromo-2-methylpropionyl bromide (230 mg, $1.0 \mathrm{mmol})$ and THF (2.0 mL) was cooled to $0{ }^{\circ} \mathrm{C}$. A solution of phenylmagnesium bromide $\left(1.0 \mathrm{M}\right.$ in $\left.\mathrm{Et}_{2} \mathrm{O}, 1.0 \mathrm{~mL}, 1.0 \mathrm{mmol}\right)$ was added dropwise. Then the reaction was stirred at room temperature for 10 hours. After the reaction was complete, the mixture was quenched with $\mathrm{H}_{2} \mathrm{O}$ and the resulting mixture was extracted with $\mathrm{CH}_{2} \mathrm{Cl}_{2}$ for 3 times. The combined organic layers were washed dried $\left(\mathrm{MgSO}_{4}\right)$, and filtered. The filtrate was concentrated under reduced pressure. The residue was purified by chromatography on silica gel.

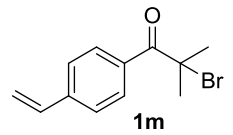

Figure S3 Synthesized $\alpha$-bromo ketone with procedure C

\section{General procedure D:}

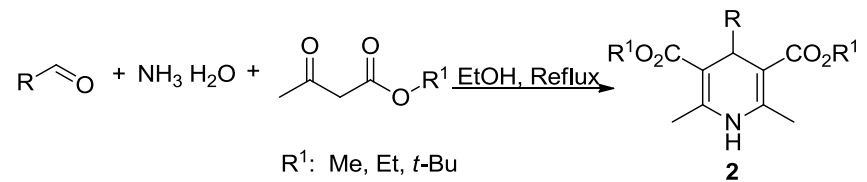

To a flask charged with $t$-butyl acetoacetate $(3.16 \mathrm{~g}, 20 \mathrm{mmol})$ (or methyl acetoacetateand ethyl acetoacetate), the aldehyde $(10 \mathrm{mmol})$ and ethanol $(20 \mathrm{~mL})$ was added ammonia aqueous solution (4.0 
$\mathrm{mL}, 28 \%, \quad 60 \mathrm{mmol})$. The mixture was heated at $70^{\circ} \mathrm{C}$ for 8 hours. The reaction was allowed to cool to room temperature. The solution was concentrated under reduced pressure. A mixture of water and $\mathrm{CH}_{2} \mathrm{Cl}_{2}$ were added to the concentrated residue and the layers were separated. The aqueous layer was extracted with $\mathrm{CH}_{2} \mathrm{Cl}_{2}$ for 3 times. The combined organic layers were washed with brine, dried $\left(\mathrm{MgSO}_{4}\right)$, and filtered. The filtrate was concentrated under reduced pressure. The residue was purified by chromatography on silica gel. Obtained solid product could be washed with PE/EA (95:5, v/v) as further purification. $\mathbf{2 a}, \mathbf{2 b}$ and $\mathbf{2 c}$ are known compounds. [ $\left.{ }^{10}\right]$

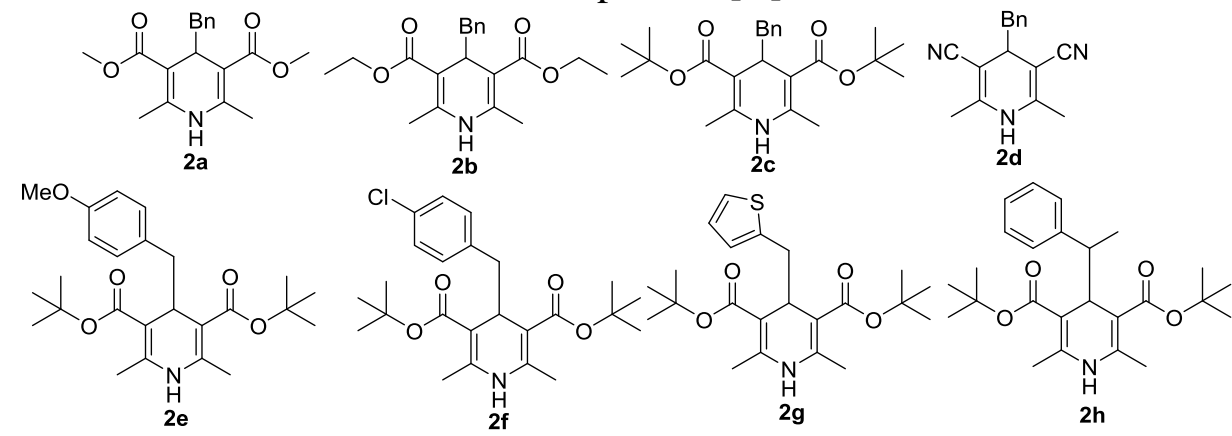

Figure S4 The synthesized substituted Hantzsch ester

\section{General procedure E:}

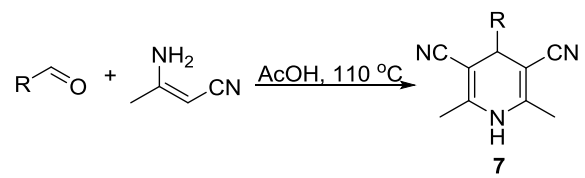

A reaction flask was charged with 3-Aminocrotononitrile $(1.6 \mathrm{~g}, 20 \mathrm{mmol})$, the aldehyde $(10 \mathrm{mmol})$, $\mathrm{AcOH}(10 \mathrm{~mL})$. The reaction was heated at $110^{\circ} \mathrm{C}$ with stirring for 3 hours. The crude reaction mixture was allowed to cool to room temperature. The reaction was diluted with water and extracted with EtOAc for three times. The combined organic layers were neutralized with saturated solution of $\mathrm{NaHCO}_{3}$ until the removal of acetic acid was achieved, washed with brine, dried over $\mathrm{MgSO}_{4}$, and filtered. The filtrate was concentrated under reduced pressure. The residue was purified by chromatography on silica gel. The obtained solid product could be washed with PE/EA (95:5, v/v) as further purification.
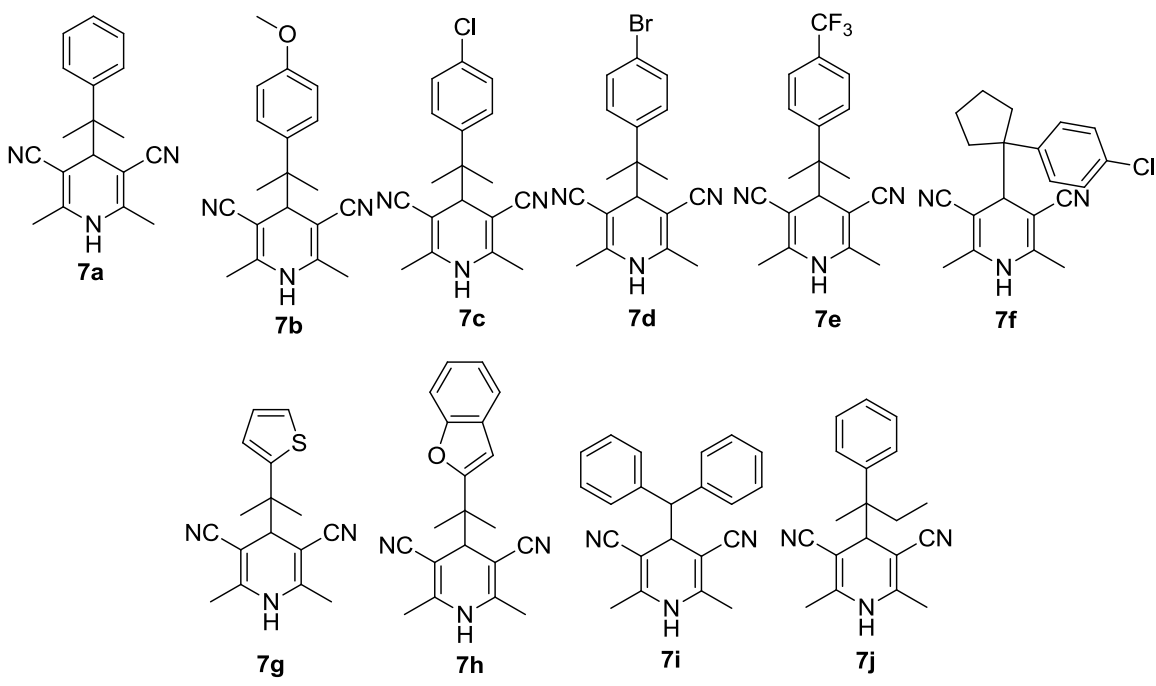
Figure S5 Synthesized Hantzsch nitriles (for $\mathbf{7} \mathbf{j}$ see modified procedure)

\section{General procedure $F$ for visible-light induced photoredox alkylation reaction with Hantzsch}

ester

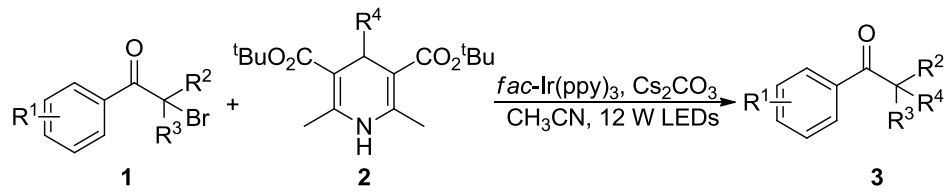

A reaction tube was charged with the $f a c-\operatorname{Ir}(\mathrm{ppy})_{3}(0.06 \mathrm{mg}, 0.1 \mathrm{~mol} \%), \mathrm{Cs}_{2} \mathrm{CO}_{3}(32.5 \mathrm{mg}, 0.1 \mathrm{mmol}$, 1.0 equiv), Hantzsch ester 2 ( $0.3 \mathrm{mmol}, 3.0$ equiv), $\alpha$-bromo-ketones 1 ( $0.1 \mathrm{mmol}, 1.0$ equiv) and $\mathrm{CH}_{3} \mathrm{CN}(2 \mathrm{~mL})$. The mixture was stirred under irradiation of $12 \mathrm{~W}$ white LED strip under nitrogen atmosphere at room temperature for the given time. Then the mixture was diluted with $\mathrm{H}_{2} \mathrm{O}$ and the resulting mixture was extracted with dichloromethane for 3 times. The combined organic layers were washed with brine, dried $\left(\mathrm{MgSO}_{4}\right)$, and filtered. The filtrate was concentrated under reduced pressure. The residue was purified by chromatography on silica gel.

\section{General procedure $G$ for visible-light induced photoredox alkylation reaction with Hantzsch}

\section{nitrile}

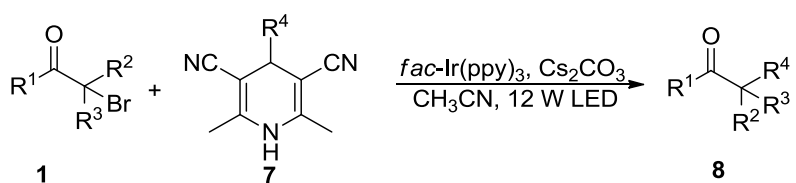

A reaction tube charged with the $f a c-\operatorname{Ir}(\mathrm{ppy})_{3}(0.256 \mathrm{mg}, 0.2 \mathrm{~mol} \%), \mathrm{Cs}_{2} \mathrm{CO}_{3}(65.0 \mathrm{mg}, 0.2 \mathrm{mmol}, 1.0$ equiv), Hantzsch nitrile 7 ( $0.6 \mathrm{mmol}, 3.0$ equiv), $\alpha$-bromo-ketone 1 ( $0.2 \mathrm{mmol}, 1.0$ equiv) and $\mathrm{CH}_{3} \mathrm{CN}$ $(4 \mathrm{~mL})$ was stirred under irradiation of $12 \mathrm{~W}$ white LED strip under nitrogen atmosphere at room temperature for the given time. Then the mixture was diluted with $\mathrm{H}_{2} \mathrm{O}$ and the resulting mixture was extracted with dichloromethane for 3 times. The combined organic layers were dried $\left(\mathrm{MgSO}_{4}\right)$, and filtered. The filtrate was concentrated under reduced pressure. The residue was purified by chromatography on silica gel. 


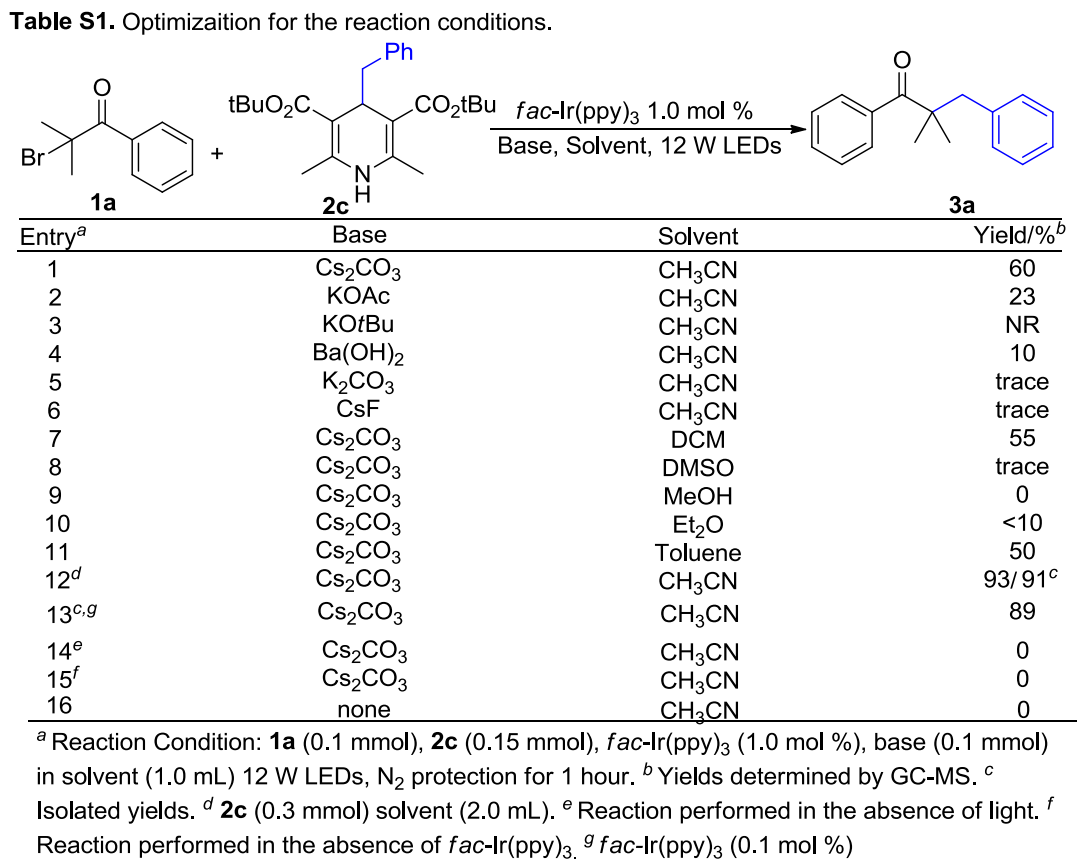

\section{Experiments on mechanism}

\subsection{Fluorescence quenching experiments}

In order to investigate the wavelength of fluorescent quenching, the UV-Vis absorption of fac- $\mathrm{Ir}(\mathrm{ppy})_{3}$ in $\mathrm{CH}_{3} \mathrm{CN}$ was measured. $374 \mathrm{~nm}$ was chosen as the irradiation wavelength . The fluorescence was recorded from the direction $90^{\circ}$ to the inlet of light at $374 \mathrm{~nm}$. (Figure S6)
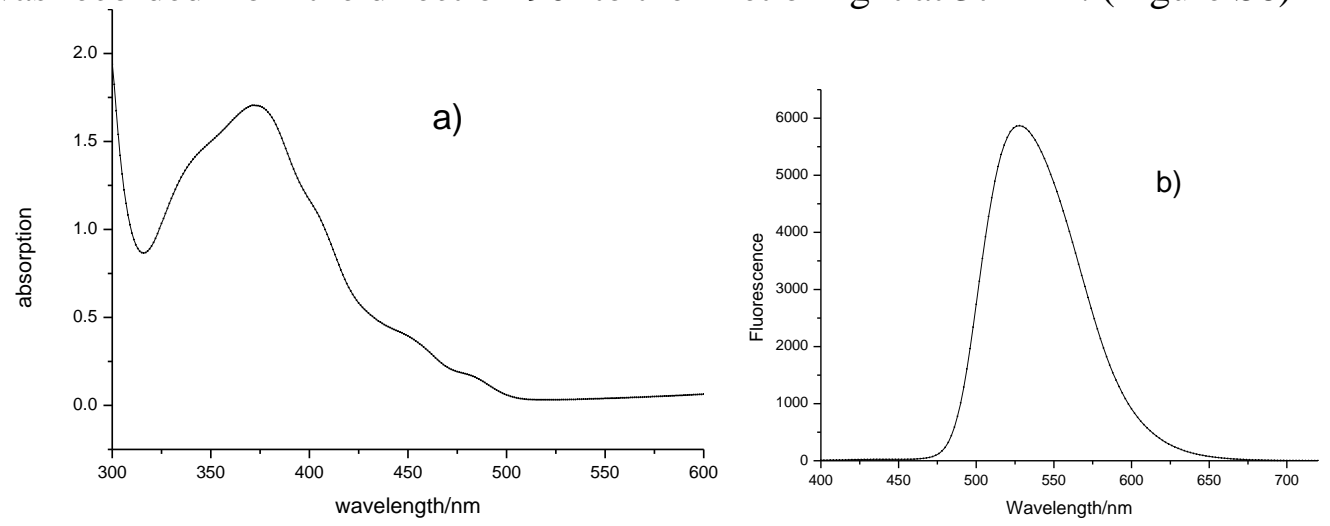

Figure S6 a) UV-Vis absorption of $f a c-\operatorname{Ir}(\mathrm{ppy})_{3}$. b) Fluorescent spectrum of $f a c-\operatorname{Ir}(\mathrm{ppy})_{3}$ with irradiation at $374 \mathrm{~nm}$.

All $\mathrm{fac}$ - $\operatorname{Ir}(\mathrm{ppy})_{3}$ solutions were irradiated at $374 \mathrm{~nm}$ and the emission intensity from 400 to $700 \mathrm{~nm}$ was recorded by F-7000 FL Spectrophotometer with EX Slit:10, EM Slit:10, PMT Voltage: 750V, Scan speed: $1200 \mathrm{~nm} / \mathrm{min}$. In a typical experiment, to a $2 \mathrm{~mL}$ solution of $f a c-\operatorname{Ir}(\mathrm{ppy})_{3}$ in $\mathrm{MeCN}$ $(0.005 \mathrm{mmol} / \mathrm{mL})$ was added $1 \mathrm{a}(0.0125 \mathrm{mmol}, 0.025 \mathrm{mmol}, 0.0375 \mathrm{mmol}, 0.05 \mathrm{mmol}$ and 0.0625 $\mathrm{mmol}$ in turn) or $2 \mathbf{c}(0.0125 \mathrm{mmol}, 0.025 \mathrm{mmol}, 0.0375 \mathrm{mmol}, 0.05 \mathrm{mmol}$ and $0.0625 \mathrm{mmol}$ in turn). 
Emission spectra of the sample were collected instantly after each addition. (Figure S7)

a) Fluorescence Quenching of $f a c-\operatorname{Ir}(\mathrm{ppy})_{3}$ by $\mathbf{1 a}$

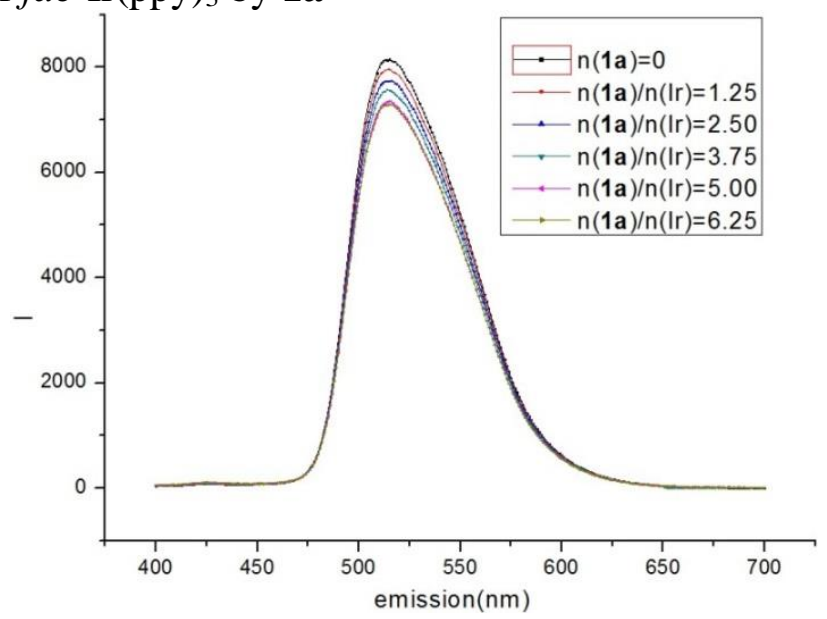

b) Fluorescence quenching of $f a c-\operatorname{Ir}(\mathrm{ppy})_{3}$ emission by $\mathbf{2 c}$

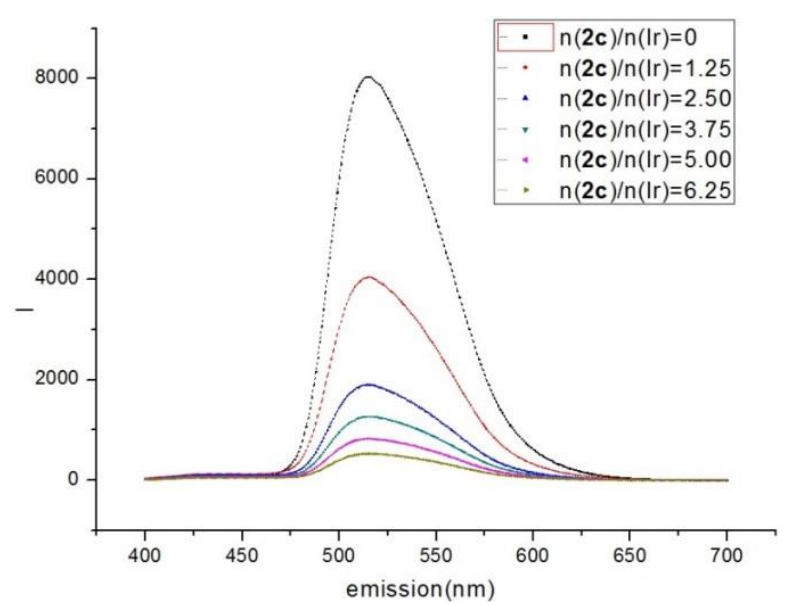

Figure S7 Fluorescence quenching of $f a c-\operatorname{Ir}(\mathrm{ppy})_{3}$ with irradiation at $376 \mathrm{~nm}$.

\subsection{Radical-capturing experiments}

(a) Radical capture was carried out with condition in Table 1, entry 3 with 4.0 equiv TEMPO.

A reaction tube was charged with the $f a c-\operatorname{Ir}(\mathrm{ppy})_{3}(0.065 \mathrm{mg}, 0.1 \mathrm{~mol} \%), \mathbf{1 a}(23.0 \mathrm{mg}, 0.1 \mathrm{mmol}, 1.0$ equiv), $2 \mathrm{c}$ (120 mg, $0.3 \mathrm{mmol}, 3.0$ equiv) and $\mathrm{Cs}_{2} \mathrm{CO}_{3}$ (32.5 mg, $0.1 \mathrm{mmol}, 1.0$ equiv) in $\mathrm{CH}_{3} \mathrm{CN}$ (2 $\mathrm{mL}$ ). The system was stirred with irradiation of $12 \mathrm{~W}$ white LED strip under nitrogen atmosphere at room temperature for 1 hour. After the reaction was completed, the solvent was removed under reduced pressure. The residue was subject to chromatography on silica gel with PE/EA $=98: 2(\mathrm{v} / \mathrm{v})$ to give the mixture of $5^{[11]}$ and $6^{[12]} 45.4 \mathrm{mg}$. The mixture of $\mathbf{5}$ and $\mathbf{6}$ was separated on semi-preparative HPLC (100\% MeOH, $1 \mathrm{~mL} / \mathrm{min}$ on YMC-Park ODS-A) to give $6(20.6 \mathrm{mg}, 83 \%)$ and $5(15 \mathrm{mg}$, ca. $50 \%$, estimated to be $25.8 \mathrm{mg}, 85 \%$ by the difference between the mixture of $\mathbf{5}+\mathbf{6}$ obtained from flash chromatography of reaction mixture and isolated $\mathbf{6}$ ). The compound $\mathbf{5}$ decomposed quickly at room temperature. The NMR spectra of 5, 6 and mixture of $\mathbf{5}$ and $\mathbf{6}$ were attached below. (Figure S8) Compound $\mathbf{6}$ is stable on GC-MS, but $\mathbf{5}$ decomposed instantly. 


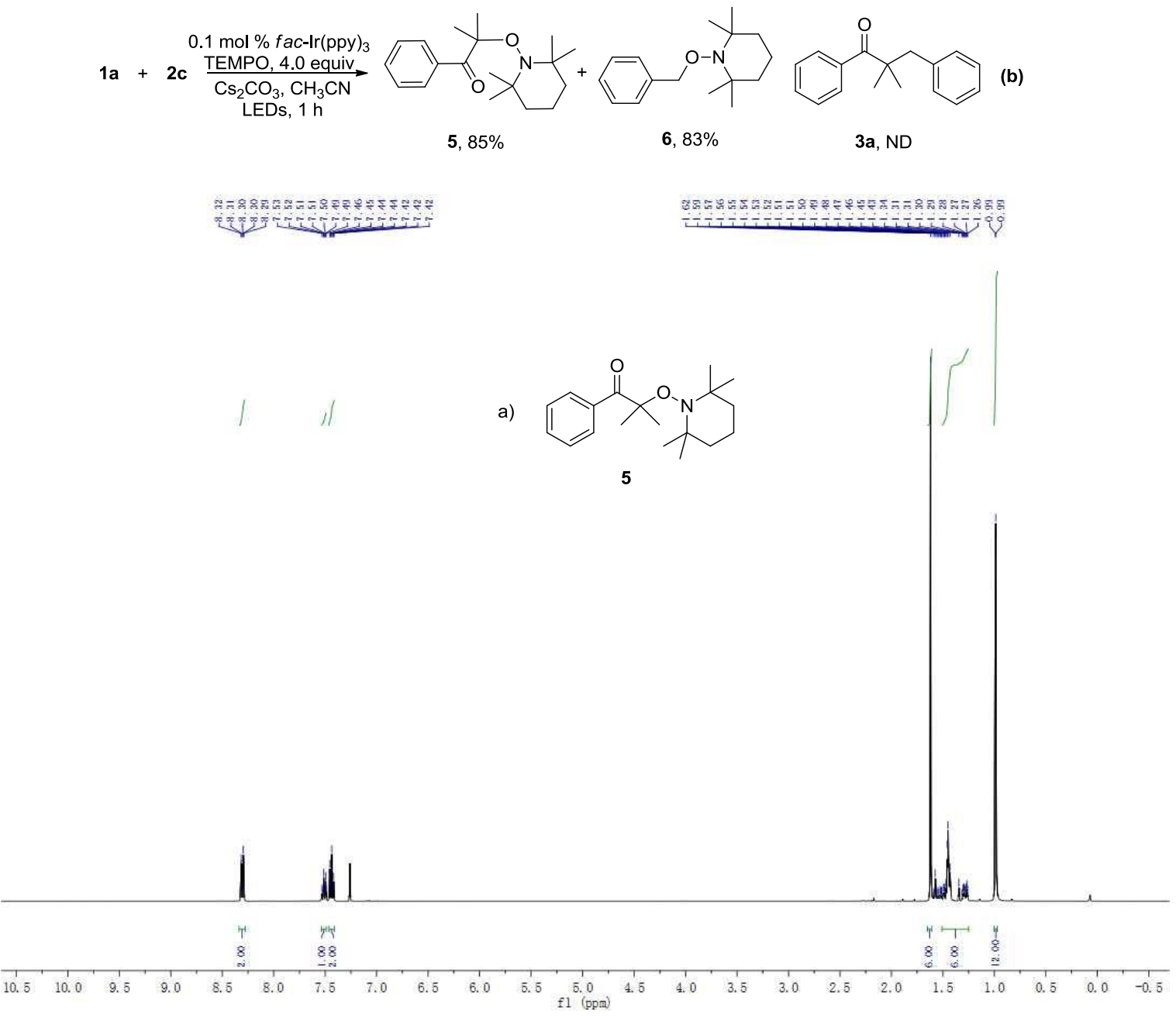

S9 


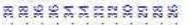

$\stackrel{\bar{x}}{\bar{i}}$

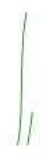

b)

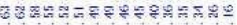

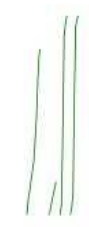

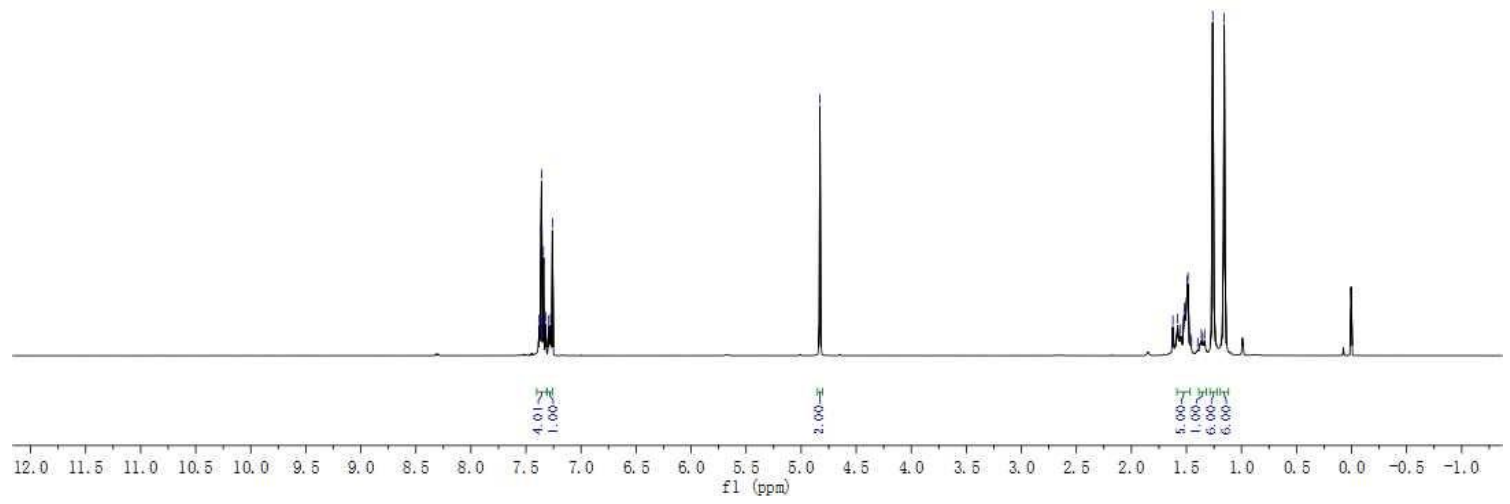

i

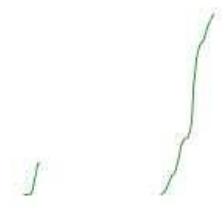

c)
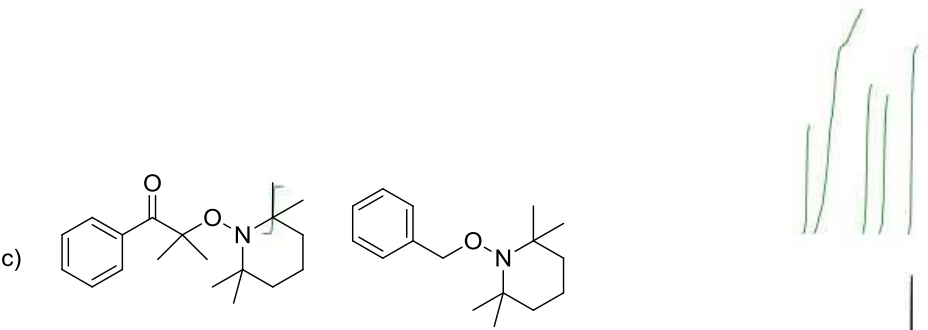

mixture of $\mathbf{5}$ and $\mathbf{6}$ after chromatography on silica gel

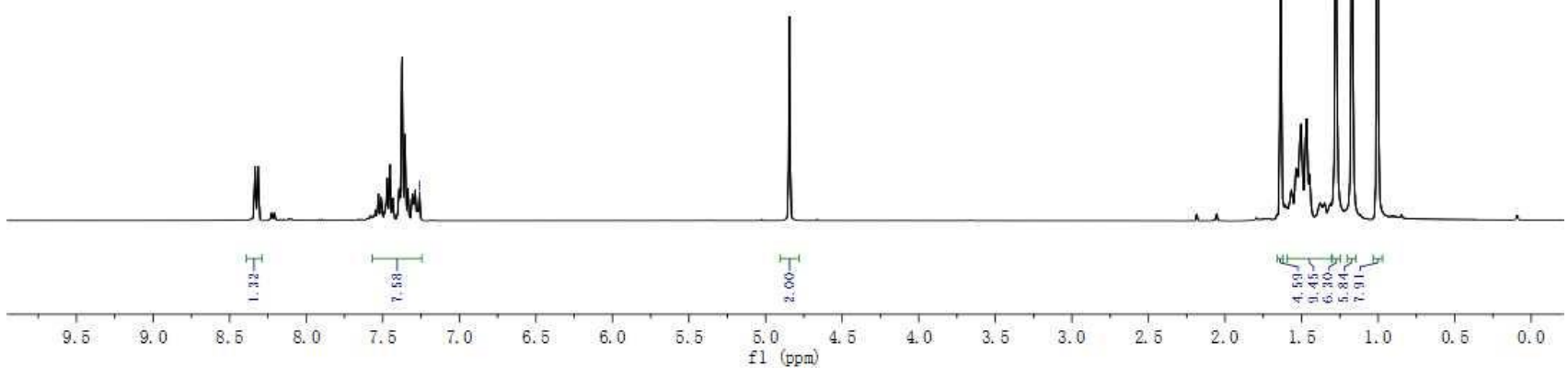

S10 
Figure S8 ${ }^{1}$ H NMR of $\mathbf{5 , 6}$ and crude mixture of 5 and $\mathbf{6}$ after flash chromotagraphy on silica gel.

The same reaction was carried out and stopped after 5, 15 and 25 minutes. The ratio of both TEMPO adduct was measured with ${ }^{1} \mathrm{H}$ NMR after flash chromatography on silica gel.

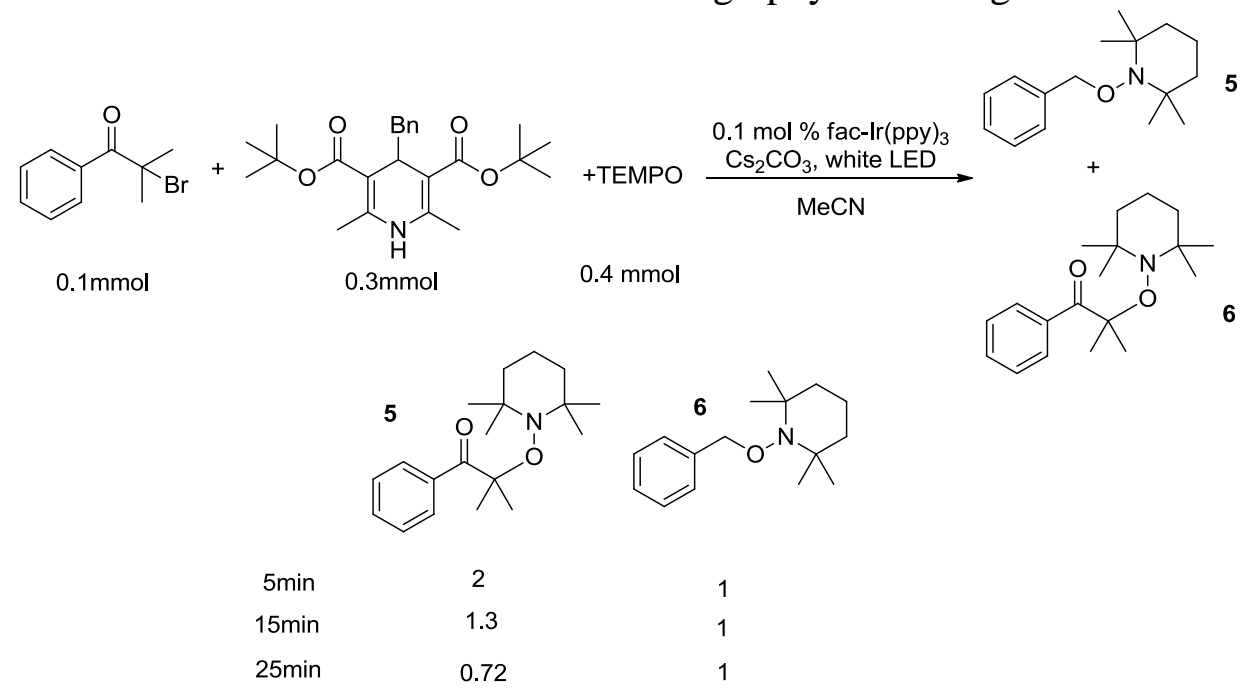

Scheme S1 Radical-capturing experiments with varied reaction time

(b) Radical-capturing experiment at $20^{\text {th }}$ minute during the reaction.

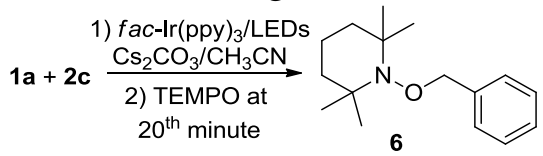

The authentic sample of $\mathbf{6}$ was synthesized with the reported procedure ${ }^{[13]}$ Benzophenone was used as internal standard to calibrate the amount of $\mathbf{6}$ in reaction system. A reaction tube was charged with the $f a c-\operatorname{Ir}(\mathrm{ppy})_{3}(0.064 \mathrm{mg}, 0.1 \mathrm{~mol} \%), \mathrm{Cs}_{2} \mathrm{CO}_{3}(32.5 \mathrm{mg}, 0.1 \mathrm{mmol}, 1.0$ equiv), $2 \mathrm{c}$ (120 mg, $0.3 \mathrm{mmol}$, 3.0 equiv), 1a (23.5, $0.1 \mathrm{mmol}, 1.0$ equiv) and $\mathrm{CH}_{3} \mathrm{CN}(2 \mathrm{~mL})$. The mixture was stirred under irradiation with $12 \mathrm{~W}$ white LED strip for 20 mins. The light was turned off and at the same time TEMPO (31.2 $\mathrm{mg}, 0.2 \mathrm{mmol}$ ) was added in one portion. The mixture was stirred for 5 minutes in the darkness. Then to the mixture was added benzophenone $(1.82 \mathrm{mg}, 0.01 \mathrm{mmol})$ and $\mathrm{CH}_{3} \mathrm{CN}$. The mixture was filtered through celite. The filtrate was analyzed with GC-MS. By comparing the ratio of peak area of benzophenone to that of $\mathbf{6}$ from plot S1, 0.013 mmol of $\mathbf{6}$ was observed (Figure S9).

GC condition to detect 6: Rxi ${ }^{\circledR}-5 \mathrm{Sil}$ column, inlet: $250{ }^{\circ} \mathrm{C}$, oven: start at $50{ }^{\circ} \mathrm{C}, 10{ }^{\circ} \mathrm{C} / \mathrm{min}$, to $250{ }^{\circ} \mathrm{C}$, hold $2 \mathrm{~min}, 20^{\circ} \mathrm{C} / \mathrm{min}$ to $280{ }^{\circ} \mathrm{C}$, hold $3 \mathrm{~min}$.

a) Prepared sample of 6 on GC-MS

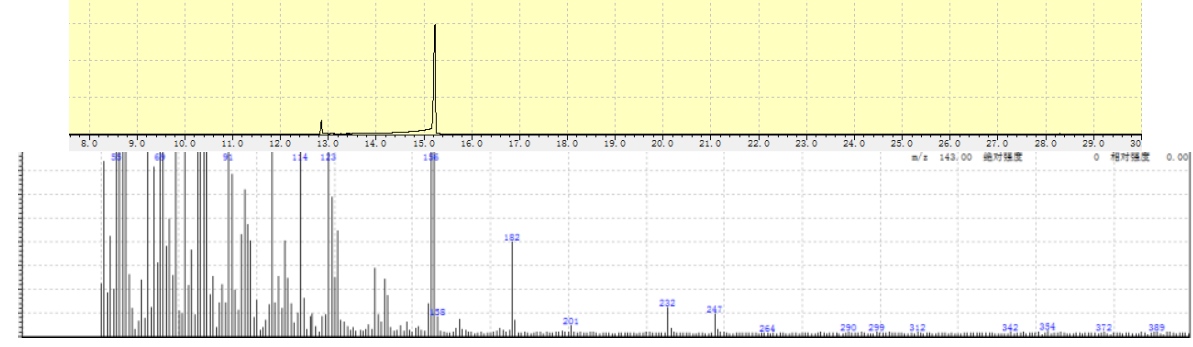


b) Commerical available benzophenone on GC-MS

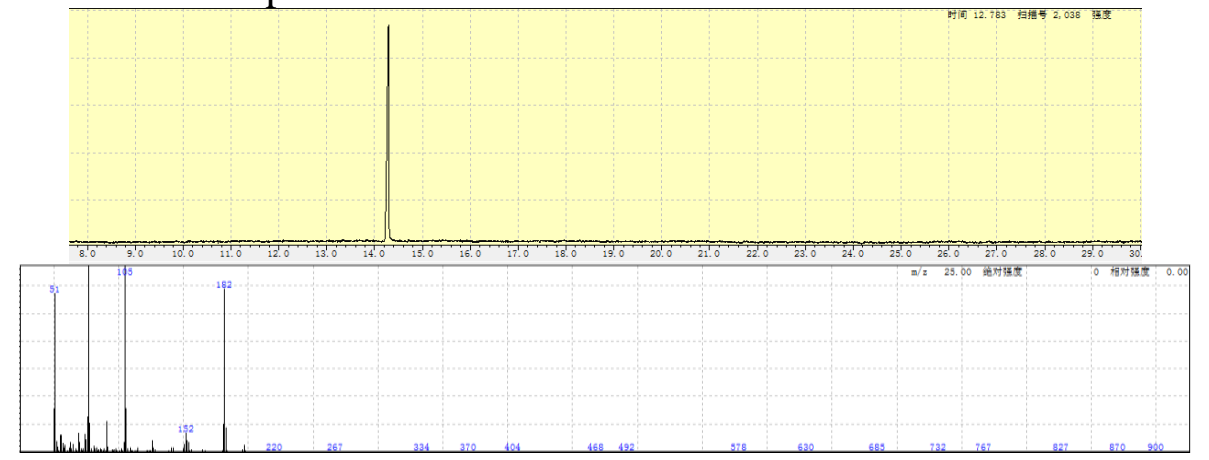

c) Calibration curve of product 6 and Benzophenone by GC-MS.

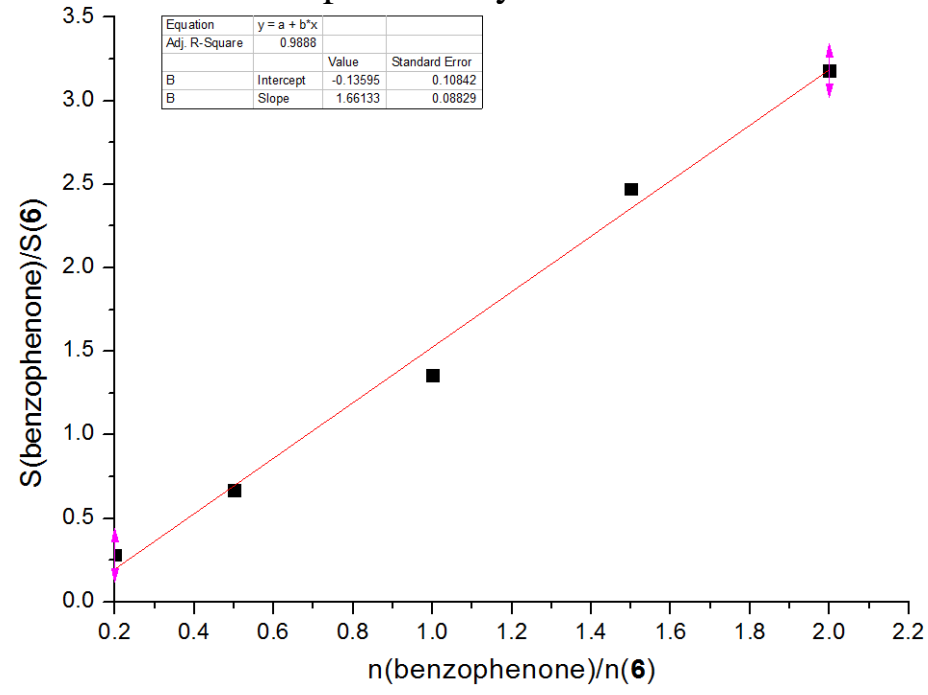

d) GC-MS chromatography of reaction quenched with TEMPO at $20 \mathrm{~min}$

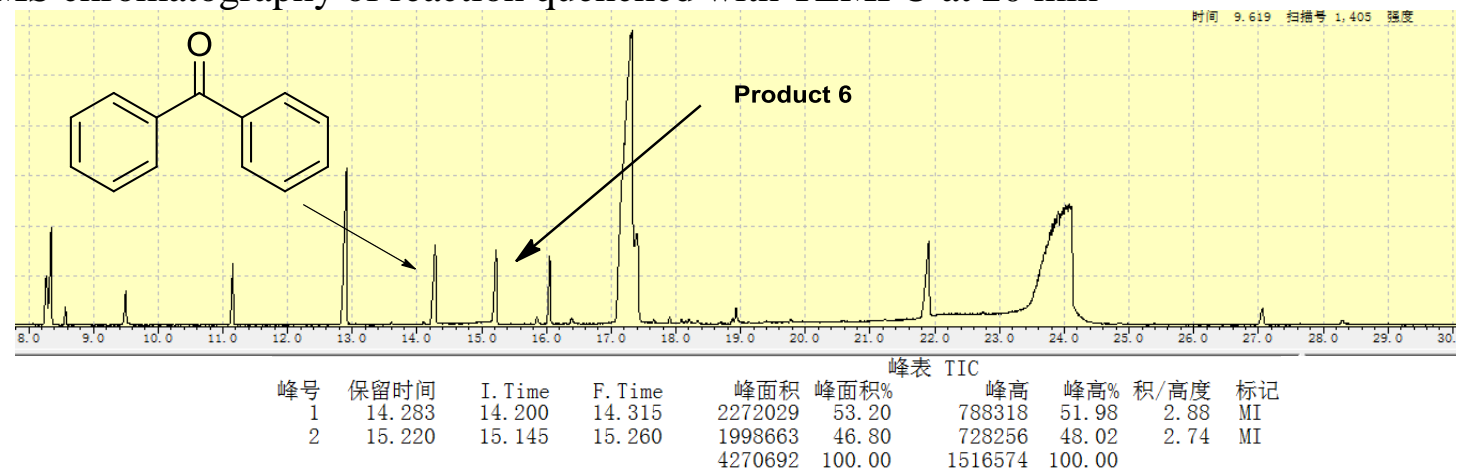

Figure S9 The GC-MS method to measure the radical species at the 20th minute.

\subsection{Spectroscopic study of Ir species and experiments with transition metal additives}

Because the loading of $f a c-\operatorname{Ir}(\mathrm{ppy})_{3}$ in standard reaction is $0.1 \mathrm{~mol} \%$ that is difficult to detect and the Hantzsch ester is 3.0 equiv that would interfere with the fluorescence of Ir species, a modified reaction with $f a c-\operatorname{Ir}(\mathrm{ppy})_{3}(6.5 \mathrm{mg}, 10 \mathrm{~mol} \%), \mathrm{Cs}_{2} \mathrm{CO}_{3}(32.5 \mathrm{mg}, 0.1 \mathrm{mmol}), \mathbf{2 c}(60 \mathrm{mg}, 1.5 \mathrm{mmol}), \mathbf{1 a}$ $(23.5 \mathrm{mg}, 0.1 \mathrm{mmol})$ and $\mathrm{CH}_{3} \mathrm{CN}(2 \mathrm{~mL})$ was carried out. The reaction gave similar conversion and 
product distribution as that with less catalyst. The UV-Vis absorption and fluorescent emission of the reaction mixture before and after the reaction was recorded. The UV-Vis absorption showed a maximum at $374 \mathrm{~nm}$. With irradiation at $374 \mathrm{~nm}$, the fluorescence spectrum of the mixture before the reaction showed no emission, suggesting the full quenching happened to Ir species and the Hantzsch ester. The fluorescence spectrum of reaction mixture after the reaction showed a major peak at $528 \mathrm{~nm}$ corresponding to the $f a c$ - $\operatorname{Ir}(\mathrm{ppy})_{3}$ an additional new peak at $454 \mathrm{~nm}$. (Figure S10)
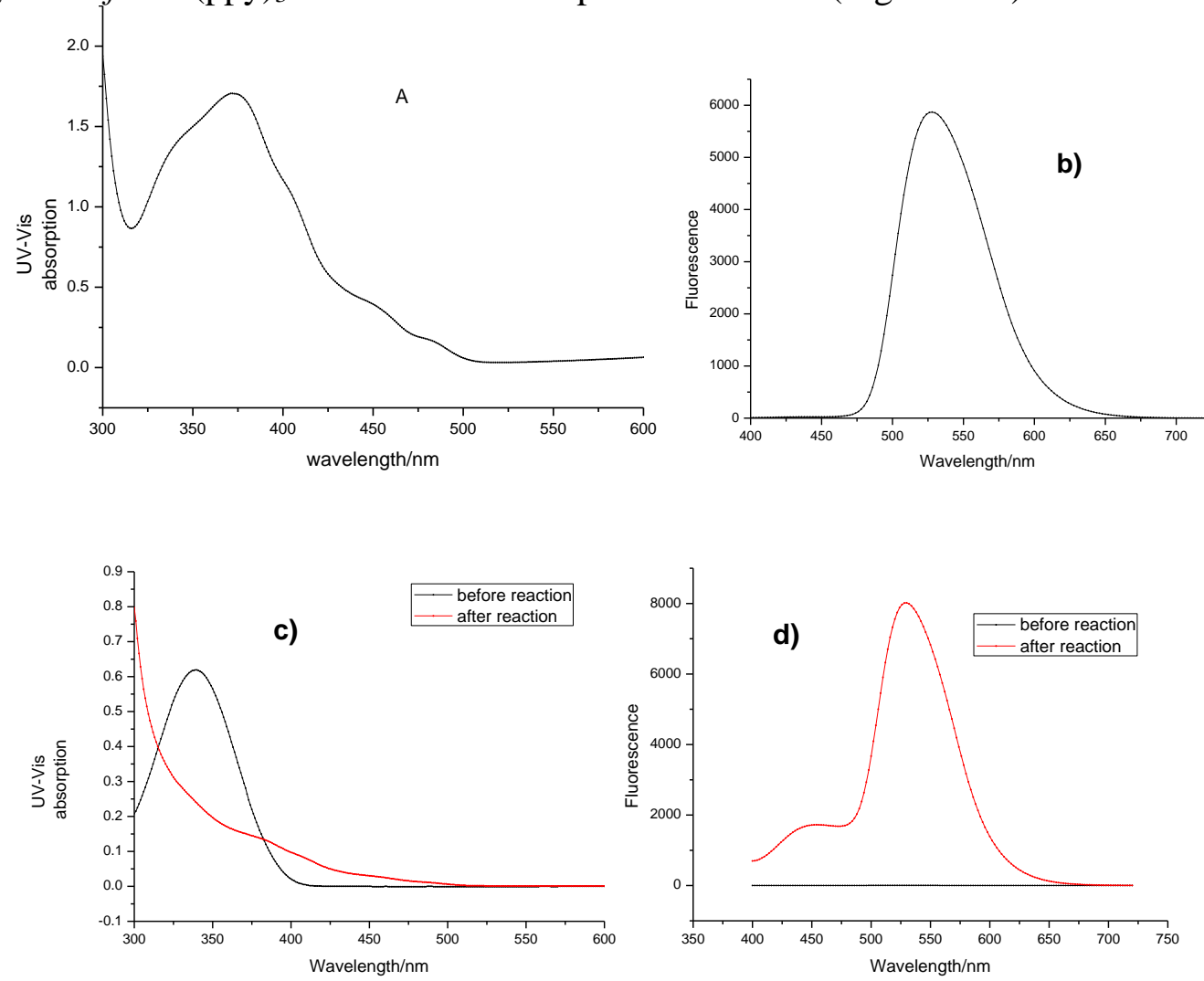

Figure S10 a) UV-Vis absorption of $f a c$-Ir(ppy) $)_{3}$. b) Fluorescence of $f a c$ - $\operatorname{Ir}(\mathrm{ppy})_{3}$ under irradiation at $374 \mathrm{~nm}$. c) UV-Vis absorption of reaction mixtures before and after reaction. d) Fluorescence (under irradiation at $374 \mathrm{~nm}$ )of reaction mixtures before and after reaction.

The ESI-MS analysis was carried out using the $f a c-\operatorname{Ir}(\mathrm{ppy})_{3}$, the mixture before the reaction and the mixture after the reaction (the above reaction). The mass for $f a c-\operatorname{Ir}(\mathrm{ppy})_{3}$ is $\mathrm{C}_{33} \mathrm{H}_{24} \mathrm{IrN}_{3} \mathrm{Na}^{-}$ $[\mathrm{M}+\mathrm{Na}]^{+}$calc. 678.1491, found 678.1447. In the mixtures before and after the reaction, the corresponding peaks exist along with some new peaks. Those might represent new Ir species. (Figure S11)

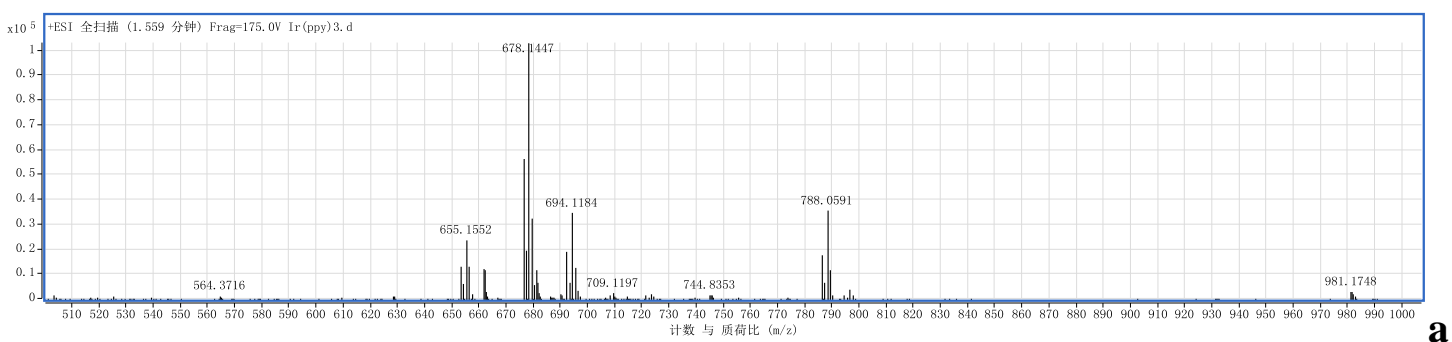



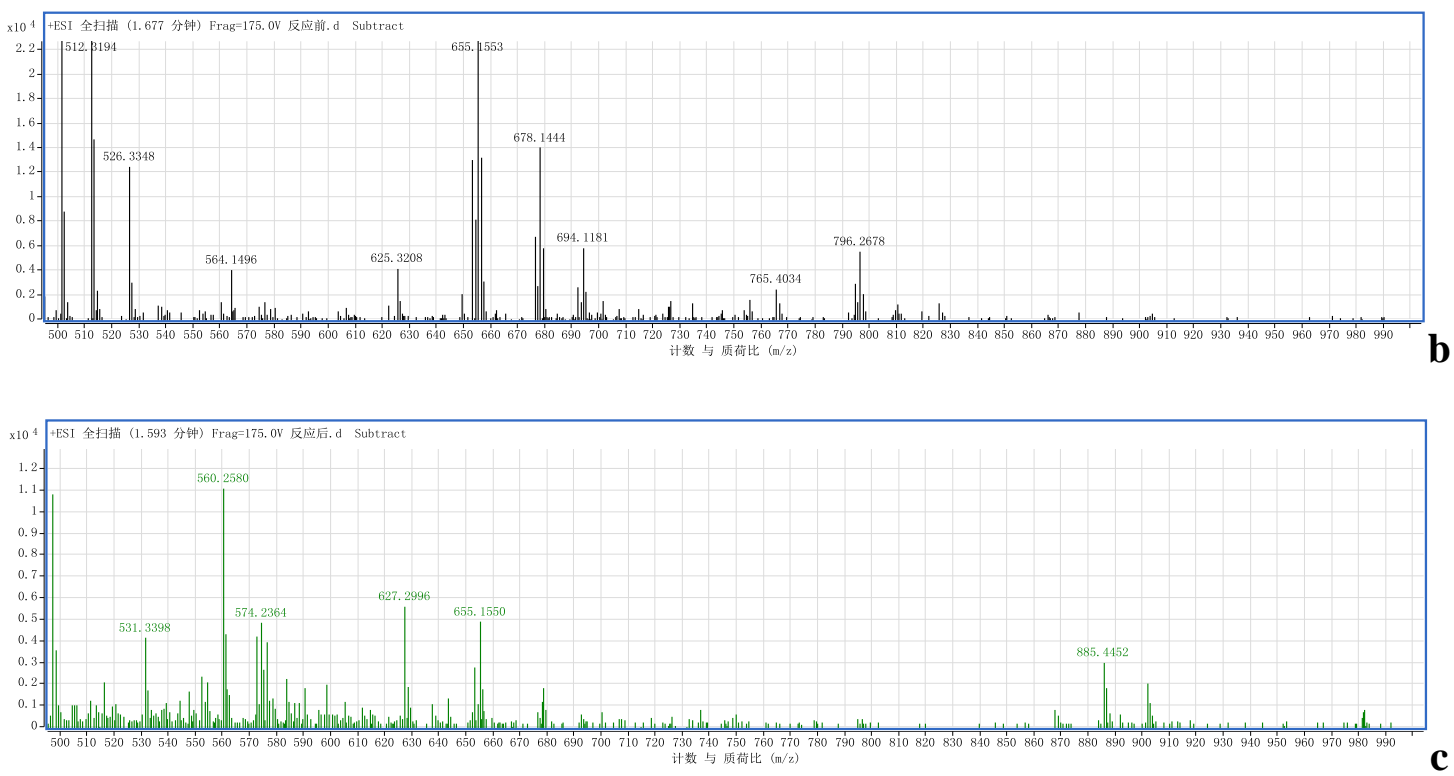

Figure S11 Mass spectra for a) $f a c-\operatorname{Ir}(\mathrm{ppy})_{3}$, b) mixture before reaction and c) mixture after the reaction.

In addition, to test whether the transition metal could promote the reaction, several reactions employing the standard reaction (table 1, entry 4) with additional transition metal complex were carried out. The varied product distribution might suggest that the transition metal species affect the reaction. (Scheme S2) So the Ir species mentioned above might catalyze the reaction within and beyond the photoredoxcycle.

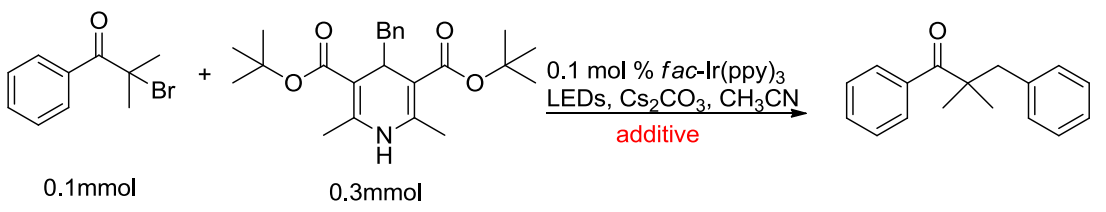

a) $10 \mathrm{~mol} \% \mathrm{Pd}(\mathrm{OAc})_{2}$

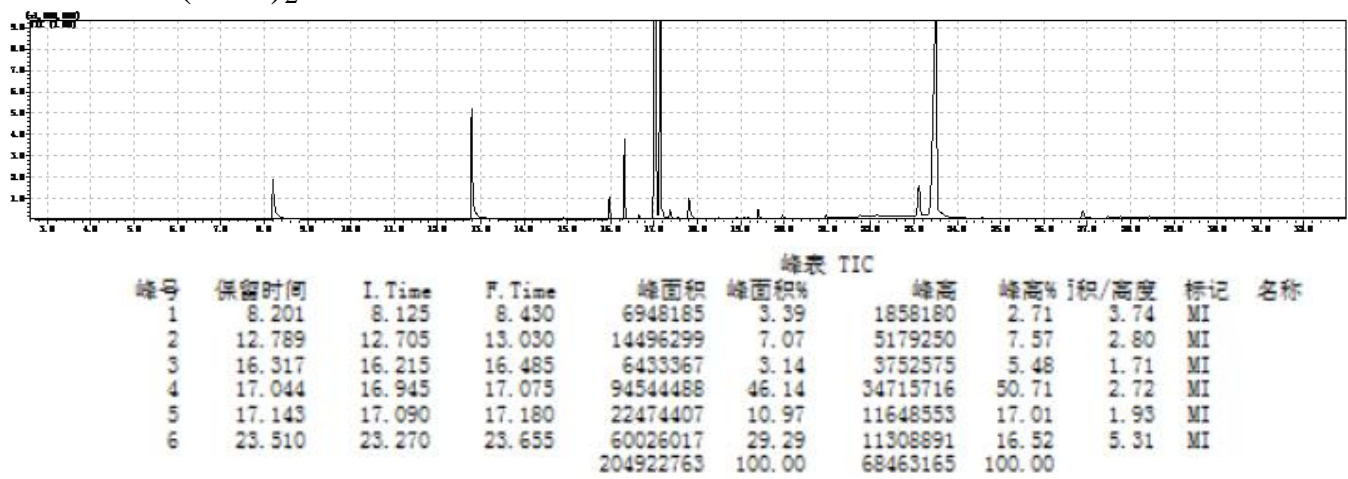

In the presence of $10 \mathrm{~mol} \% \mathrm{Pd}(\mathrm{OAc})_{2}$, peak at 21.8 minute decreased.

b) $10 \mathrm{~mol} \% \mathrm{NiCl}_{2} \cdot \mathrm{dtbbpy}$ 


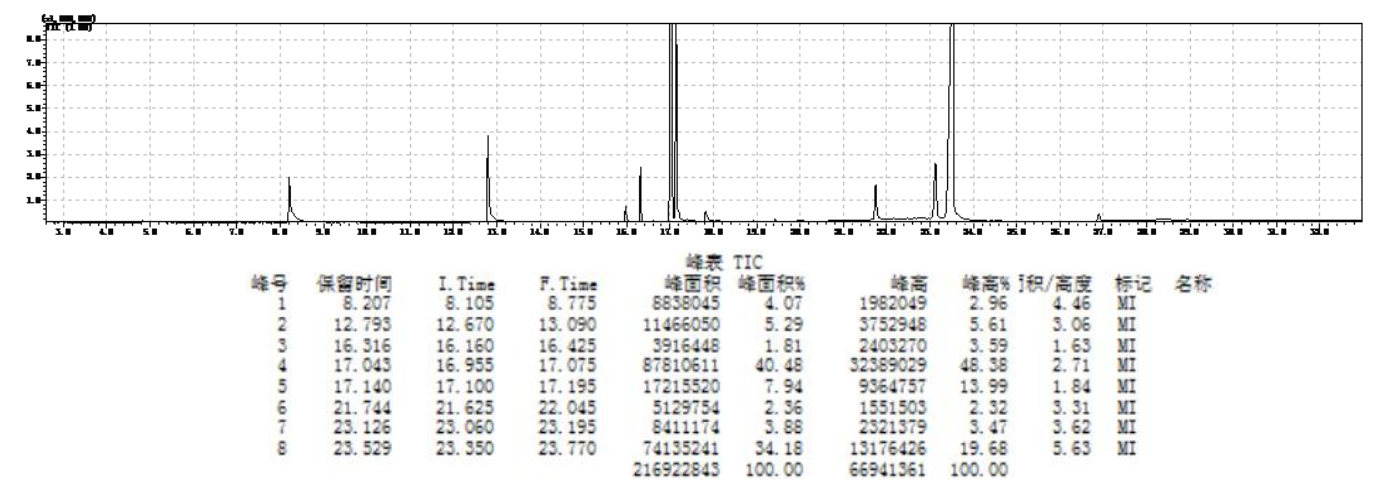

In the presence of $10 \mathrm{~mol} \% \mathrm{NiCl}_{2} \cdot \mathrm{dtbbpy}$, the peak at 8.4 minute (isobutyrophenone) increased.

c) Without additional catalyst

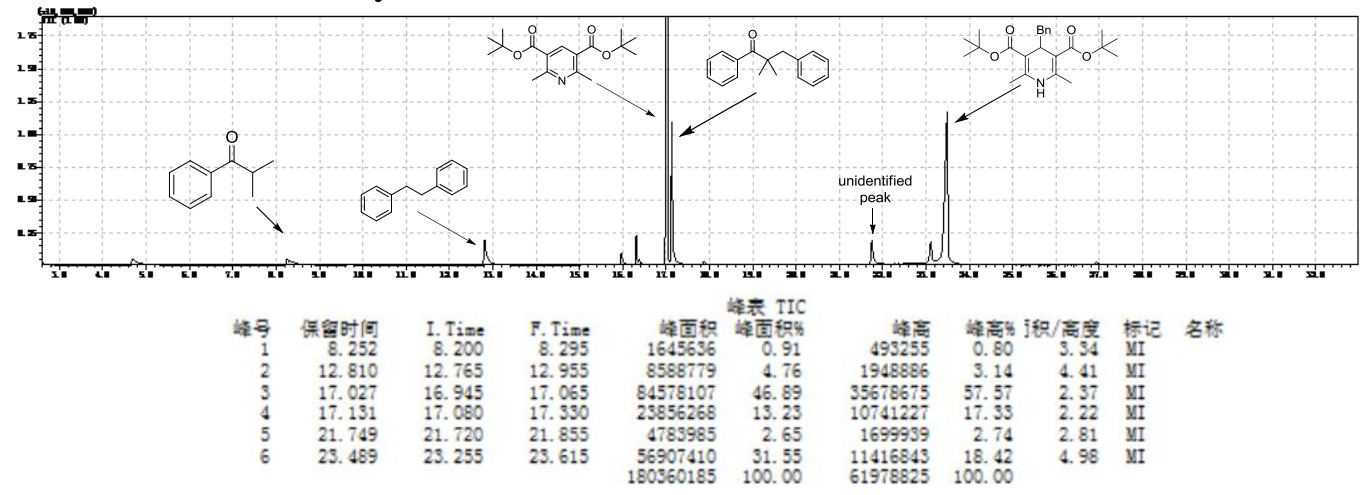

Scheme S2 Standard reaction with additional transition metal.

\subsection{Isolation of dimerized benzyl radical from standard reaction}

The standard reaction employed conditions in Table 1, entry 4): fac- $\operatorname{Ir}(\mathrm{ppy})_{3}(0.064 \mathrm{mg}, 0.1 \mathrm{~mol} \%)$, $\mathrm{Cs}_{2} \mathrm{CO}_{3}$ (32.5 mg, $0.1 \mathrm{mmol}, 1.0$ equiv), $2 \mathrm{c}(120 \mathrm{mg}, 0.3 \mathrm{mmol}, 3.0$ equiv) and $\mathbf{1 a}(23.5 \mathrm{mg}, 0.1 \mathrm{mmol}$, 1.0 equiv) in $\mathrm{CH}_{3} \mathrm{CN}(2 \mathrm{~mL})$. This reaction was carried out and purified by chromatography on silica gel. The 1,2-diphenylethane $9.1 \mathrm{mg}$, ca. $0.05 \mathrm{mmol}$ was isolated with petroleum ether as eluent. A background reaction was carried out using the above condition, except without 1a. It was found the Hantzsch ester $2 \mathbf{c}$ decomposed and gave the ca. $0.02 \mathrm{mmol}$ 1,2-diphenylethane as well.
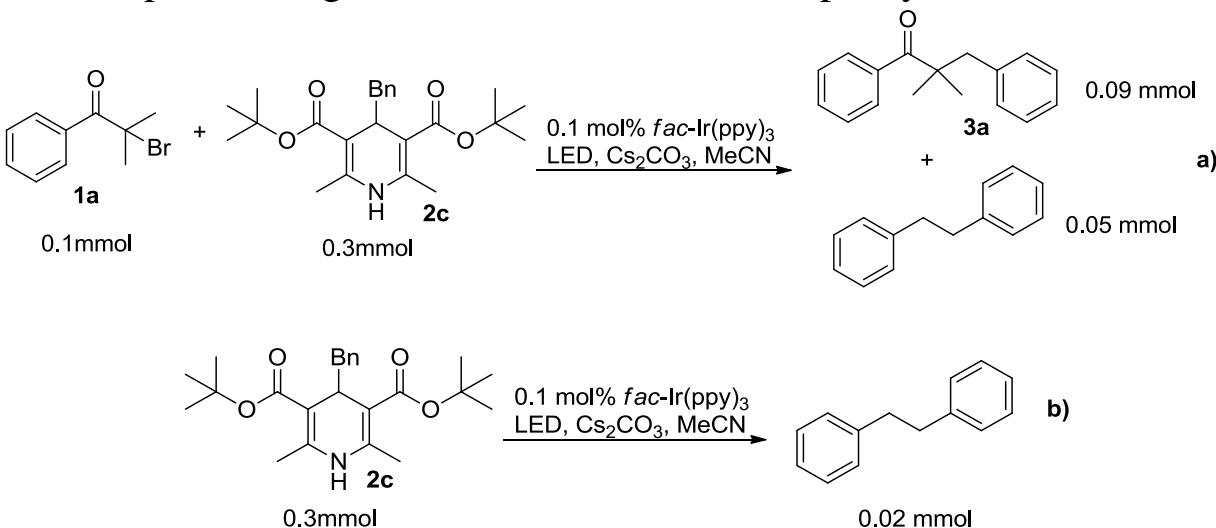

b)

Scheme S3 The dimerization of benzyl radical. a) The standard reaction. b) The standard reaction 
without 1a.

\subsection{Reactions with additional electrophiles}

The reaction might proceed via the coupling involving anionic-cationic species. In order to capture the anionic species, two reactions involving electrophiles were carried out employing the condition in Table 1, entry 3 with 1.0 equiv electrophile. (Scheme S4) The nucleophilic addition product to 4-methylbenzylbromide or benzaldeyde was not detected on GC-MS.

a) GC-MS Chromatography of reaction in Table 1, entry3
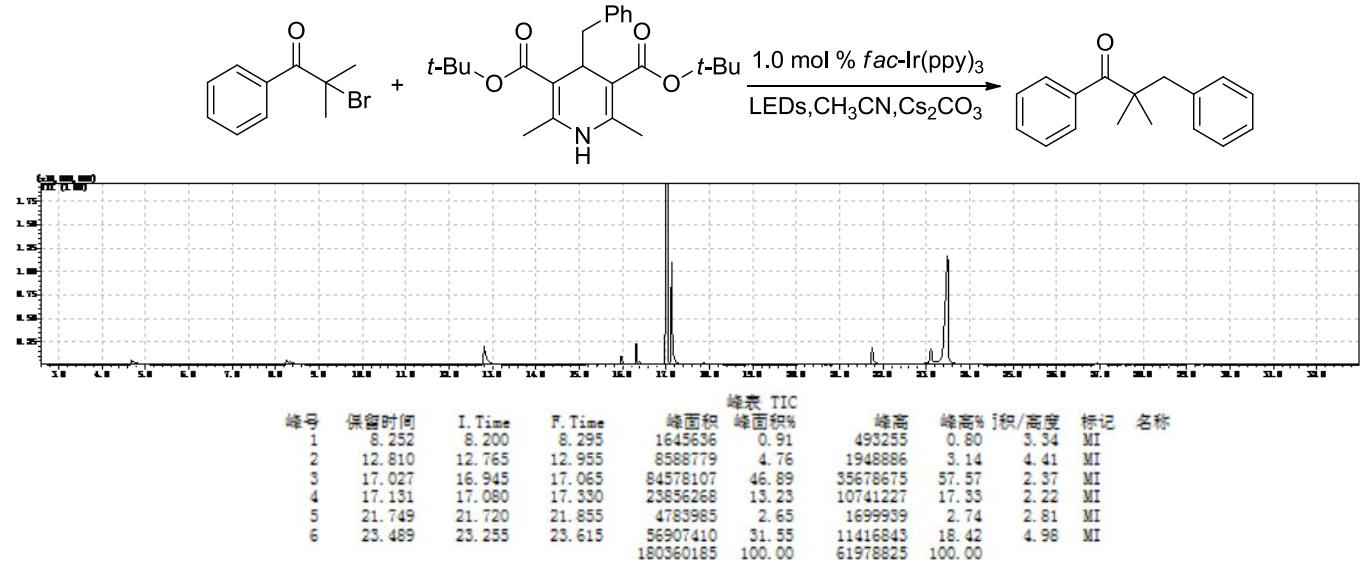

b) With 1.0 equiv 4-methyl-benzylbromide as additive (retention time was about 8.3 mins)
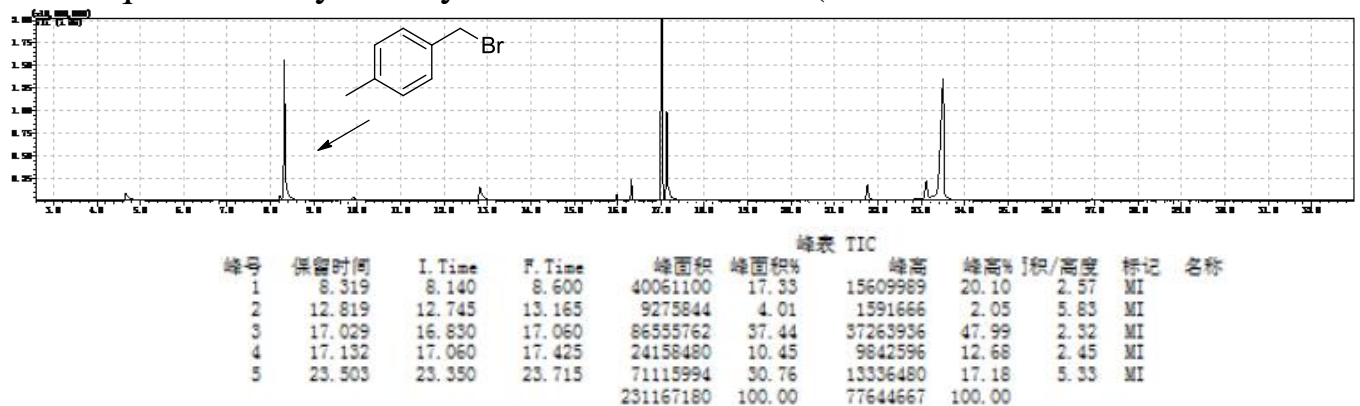

峰亦 IIC

\begin{tabular}{|c|c|c|c|}
\hline 峰言 & 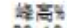 & 2/留 & 样记 \\
\hline 15609989 & 20.10 & 2. 57 & XI \\
\hline 1591666 & 2.05 & 5. 83 & YI \\
\hline 37263936 & 47.99 & 2. 32 & YI \\
\hline 9842596 & 12. 68 & 2. 45 & XI \\
\hline 13336480 & 17. 18 & 5. 33 & VI \\
\hline & 100.00 & & \\
\hline
\end{tabular}

c) With 1.0 equiv benzaldehyde as additive (retention time was about $5.7 \mathrm{~min}$ )
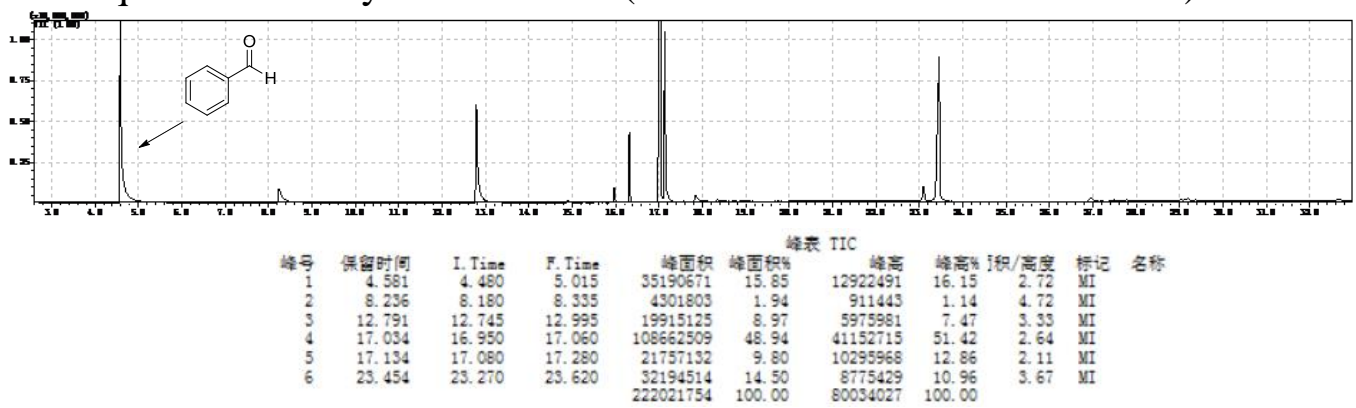

Scheme S4 The reactions with additional electrophile.

\subsection{Reaction to investigate if a radical chain pathway was involved}

A reaction tube was charged with $1 \mathbf{a}(23 \mathrm{mg}, 0.1 \mathrm{mmol}), \mathbf{2 c}(120 \mathrm{mg}, 0.3 \mathrm{mmol}), \mathrm{AIBN}(32.8 \mathrm{mg}$, 
$0.2 \mathrm{mmol}), \mathrm{Cs}_{2} \mathrm{CO}_{3}(32.5 \mathrm{mg}, 0.1 \mathrm{mmol})$ and toluene $(2 \mathrm{~mL})$ under a nitrogen atmosphere. The mixture was stirred at $80{ }^{\circ} \mathrm{C}$ for 1 hour. Then the reaction system was analyzed with GC-MS, and no target product 3a was found, suggesting the chain pathway was not favored. (Scheme S5)

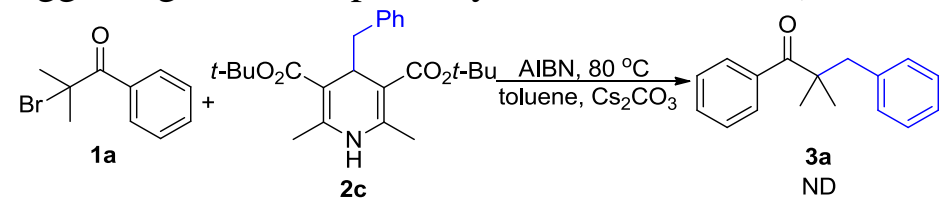

Scheme S5 Reaction using radical initiator.

\subsection{Reactions employing analougues of $1 \mathrm{a}$ with less hinderance}

The reactions (Table 1, entry 3) were carried out using analogues of 1a with less hinderance. The reactions were analyzed with GC-MS. The $\alpha$-bromoacetophenone gave the reaction more complex than that from 1a. The $\alpha$-methyl- $\alpha$-bromoacetonphenone was used the reaction and gave the desired product along with the dimer of radical derived from $\alpha$-methyl- $\alpha$-bromoacetonphenone. In comarison, the standard reaction (table 1, entry 3) was carried out as well, a peak at 21.8 min was observed, which was supposed to be the dimer of isobutyrophenone radical by retention time. By mass, it was difficult to characterize. During the purification, such dimer was not isolated.(Scheme S6)

a)
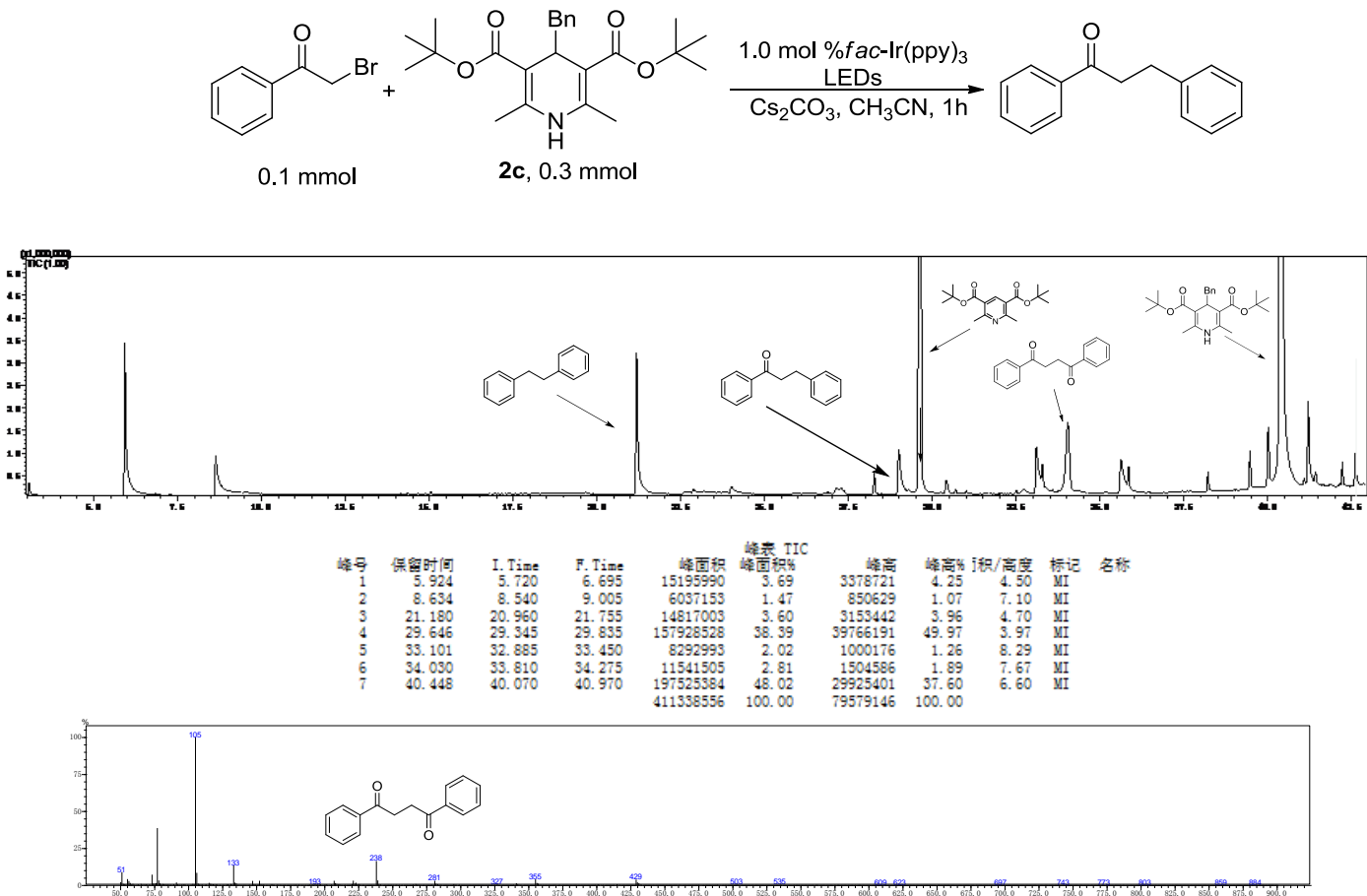

b)

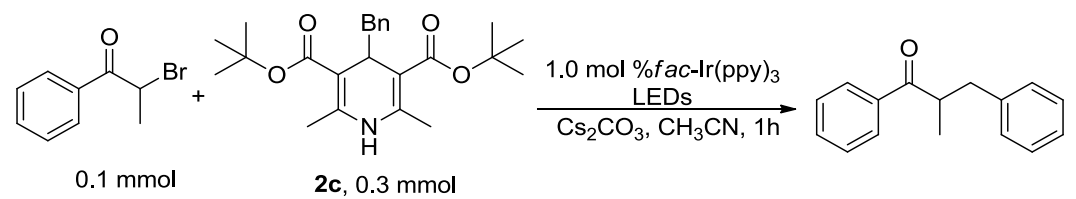



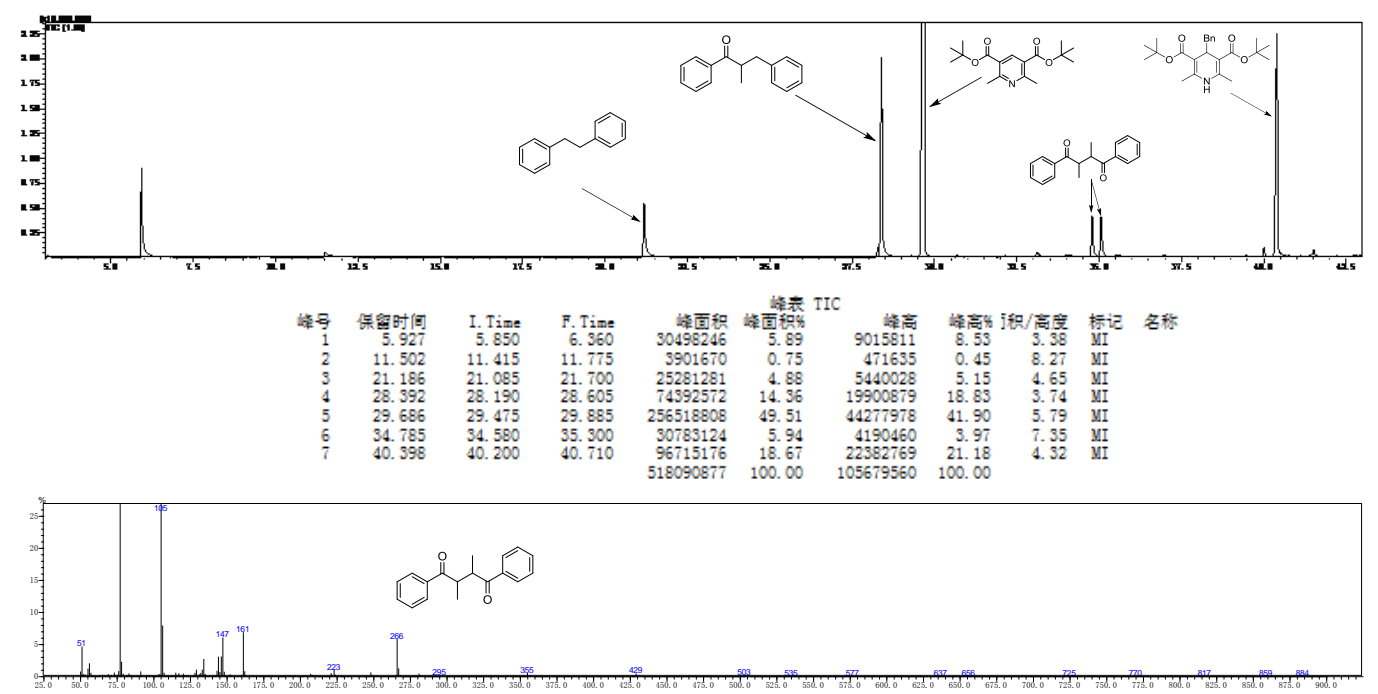

c)

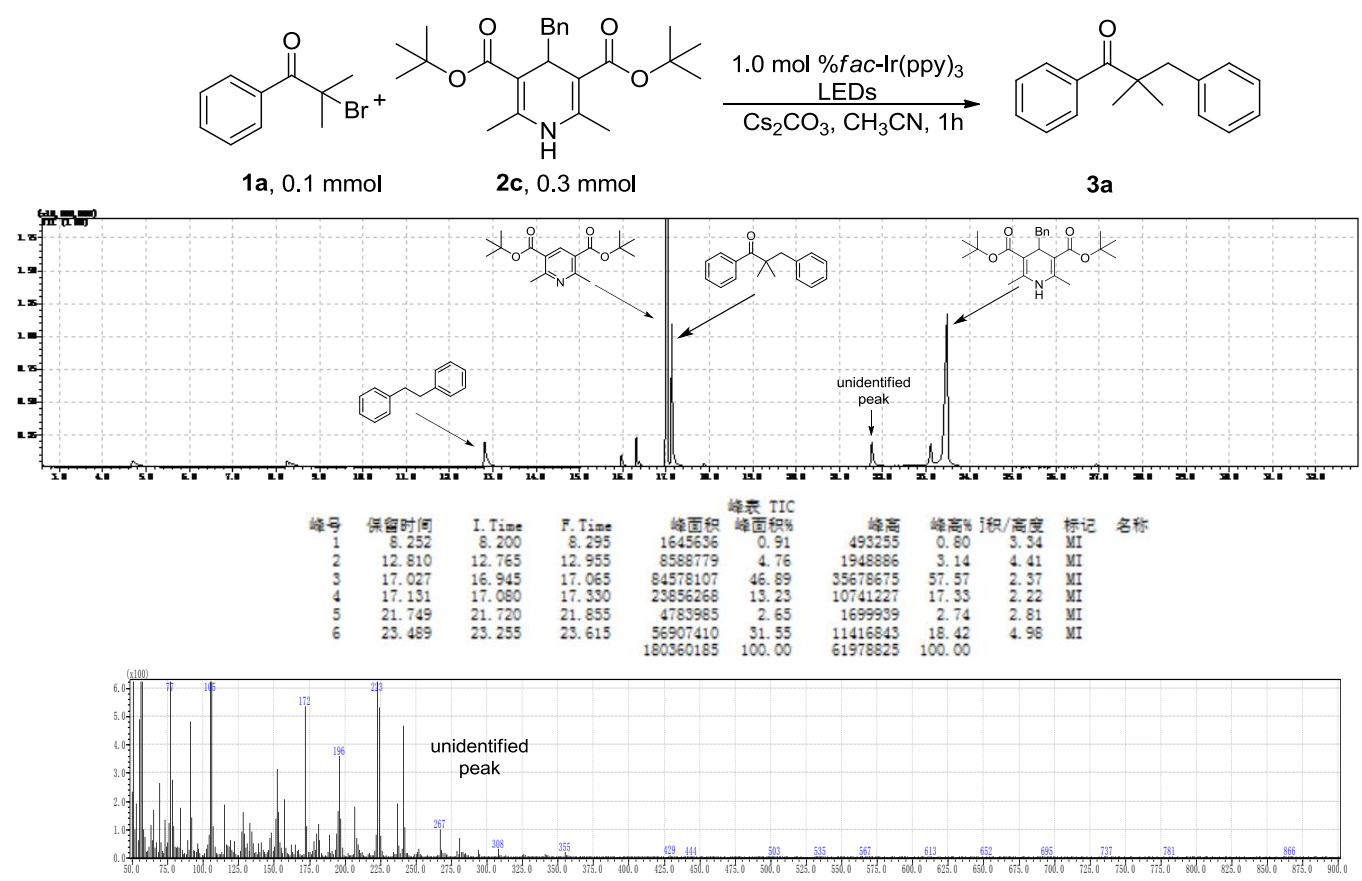

Scheme S6 The reaction with analouges of 1a with less hinderance.

\subsection{Radical clock experiment and reactions with hydride donors}

Preparation of 2-bromo-2-cyclopropyl-1-phenylpropan-1-one as a radical clock substrate was attempted at first. However, the bromination of 2-cyclopropyl-1-phenylpropan-1-one with different bromination reagents gave complex mixture. So 2-bromo-2-cyclopropyl-1-phenylethanone was brominated to give compound 1y as the substrate for the photoredox alkylation reaction. The fraction with similar $R_{f}$ to $\mathbf{3 a}$ from chromatography of reaction products was isolated. However, the product was complex, judging by ${ }^{1} \mathrm{H}$ NMR (Scheme S7). The cyclopropyl might open and side reaction might happen. 


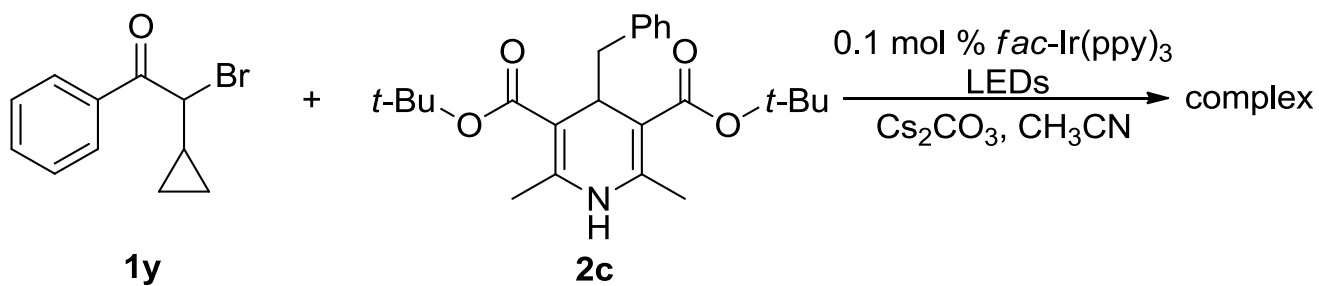

The ${ }^{1} \mathrm{H}$ NMR of mixture with similar $\mathrm{R}_{\mathrm{f}}$ to $\mathbf{3 a}$ isolated from the reaction with $1 \mathrm{y}$ as substrate

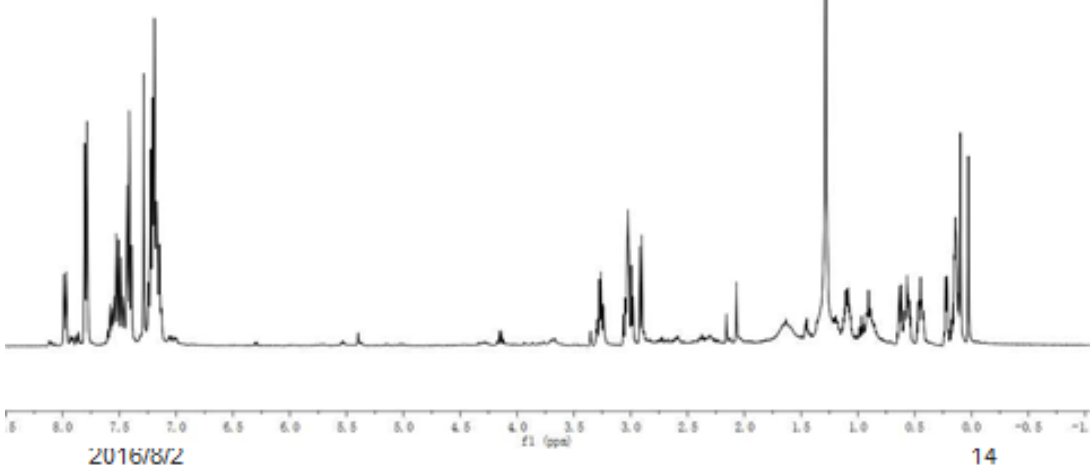

Scheme S7 Radical clock experiment.

Hydride donors, such as $\mathrm{Ph}_{3} \mathrm{SiH}, n$-BuSnH and diethyl Hantzsch ester, were used as additives in the standard reaction (Table 1 , entry 4). In the case of $\mathrm{Ph}_{3} \mathrm{SiH}$, the reaction gave more isobutyrophenone. When $n$ - $\mathrm{Bu}_{3} \mathrm{SnH}$ was applied, neither substrate 1a nor product 3a could be detected. When diethyl Hantzsch ester was applied, the isobutyrophenone was detected as a major product along with the 3a (Scheme S8).

a)

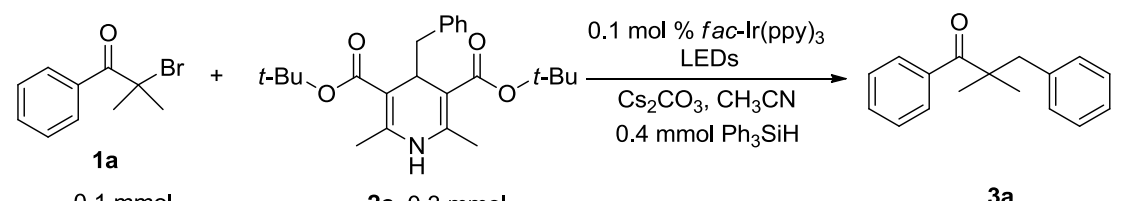

$0.1 \mathrm{mmol}$

2c, $0.3 \mathrm{mmol}$

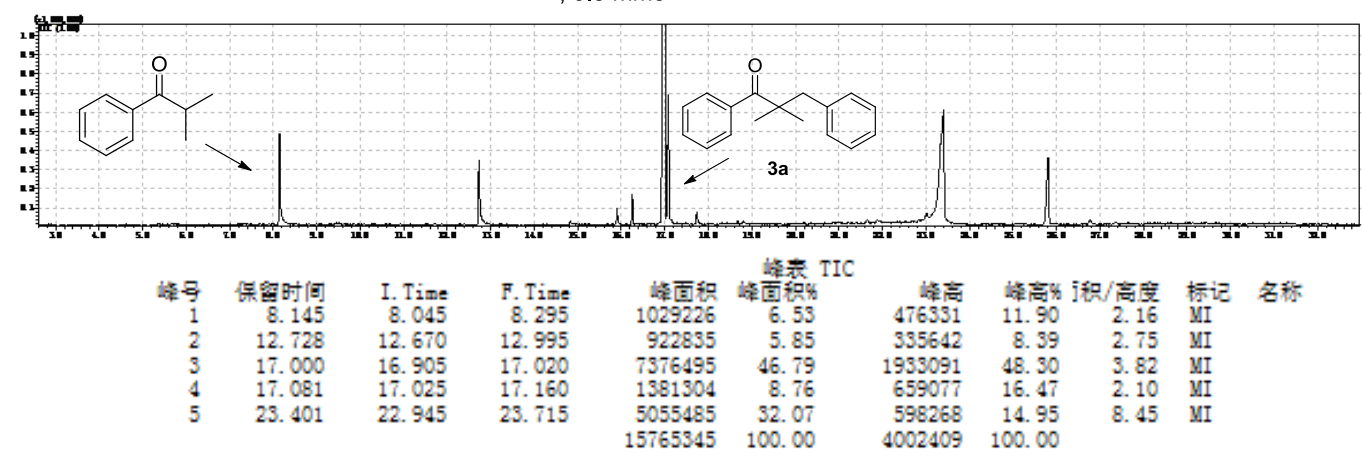

b) 

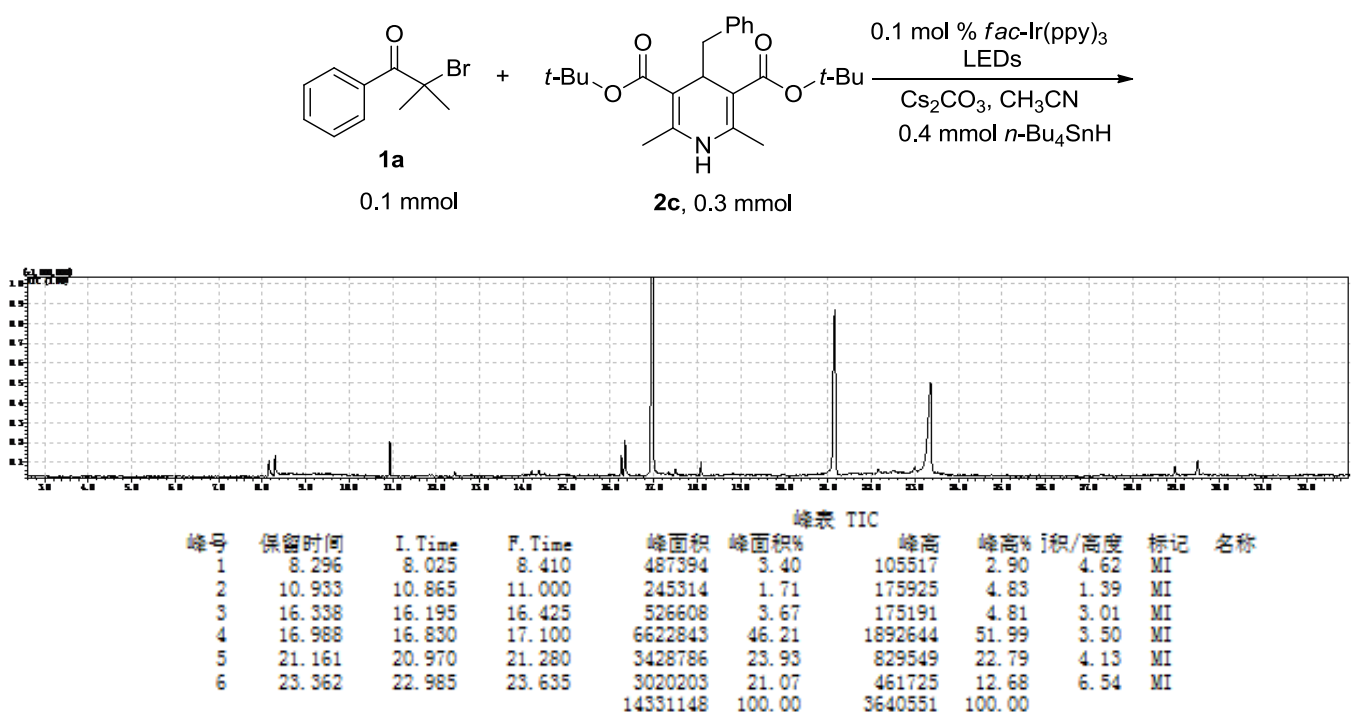

c)
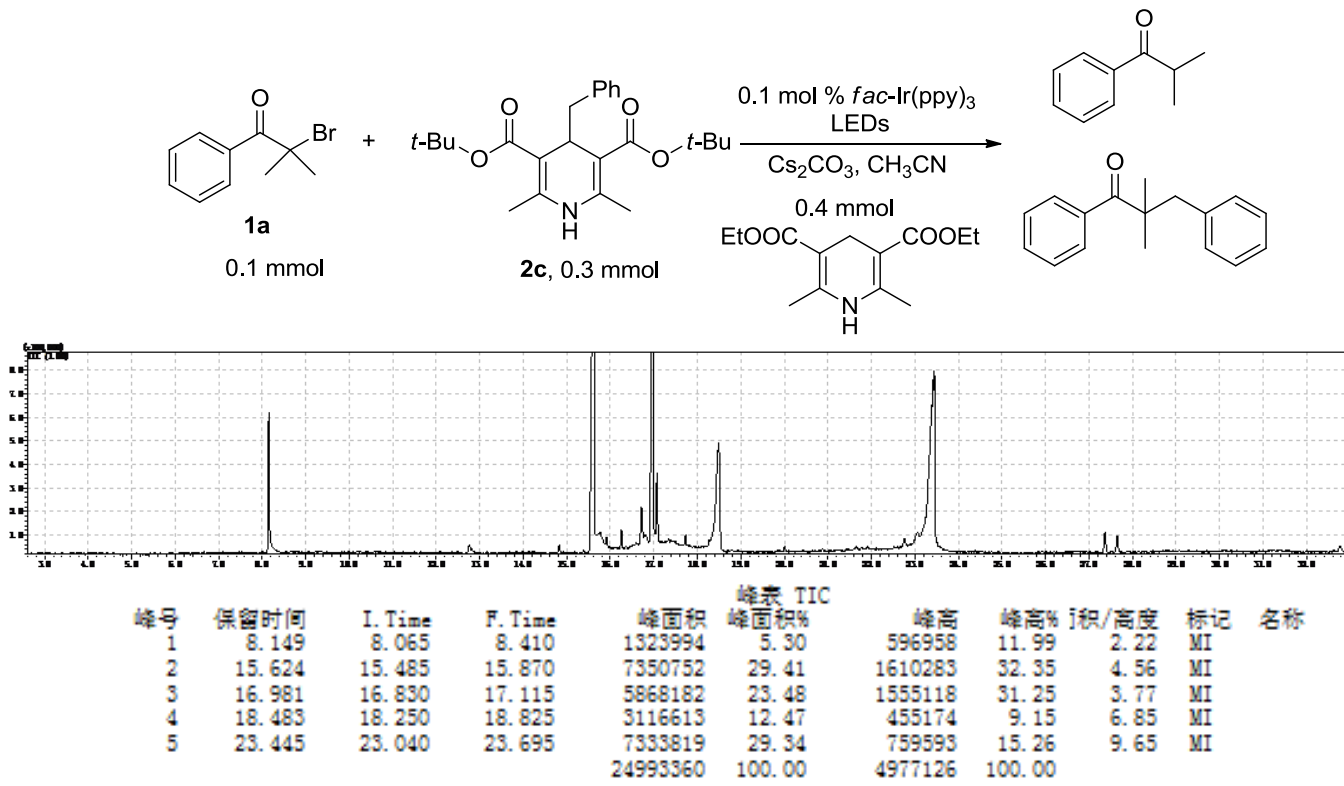

Scheme S8 Reaction with hydride donors as additive.

\subsection{Reaction of Hantzsch ester and bromobenzene in the presence of transition metal} catalysts

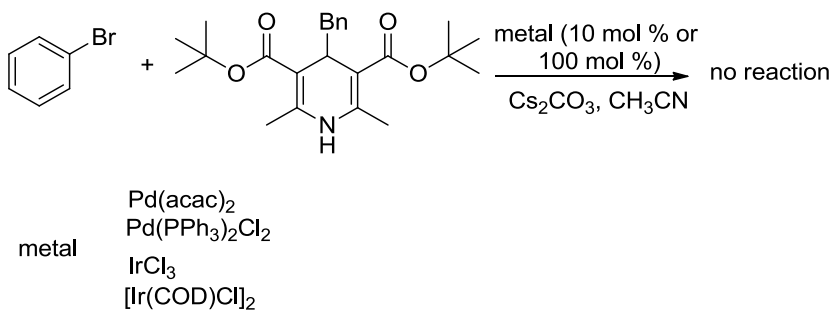

To a reaction vial charged with $2 \mathrm{c}(120 \mathrm{mg}, 0.3 \mathrm{mmol})$, bromobenzene $(15.7 \mathrm{mg}, 0.1 \mathrm{mmol}), \mathrm{Cs}_{2} \mathrm{CO}_{3}(32.5 \mathrm{mg}, 0.1$ mmol) and catalyst $(10 \mathrm{~mol} \%)$ was added $\mathrm{CH}_{3} \mathrm{CN}(2 \mathrm{~mL})$. The mixture was stirred at rt for 1 hours. The diluted reaction mixture was analyzed with GC-MS.

Another reaction with $2 \mathrm{c}(12 \mathrm{mg}, 0.01 \mathrm{mmol})$, bromobenzene $(1.6 \mathrm{mg}, 0.01 \mathrm{mmol}), \mathrm{Cs}_{2} \mathrm{CO}_{3}(3.2 \mathrm{mg}, 0.01 \mathrm{mmol})$ 
and catalyst ( $0.01 \mathrm{mmol}$ ) in $0.4 \mathrm{~mL} \mathrm{CH}_{3} \mathrm{CN}$ was stirred at $\mathrm{rt}$ for 1 hours. The diluted reaction mixture was analyzed with GC-MS.

In the reactions employing $10 \mathrm{~mol} \%$ catalyst or $100 \mathrm{~mol} \%$ catalyst, no significant conversion took place.

$\operatorname{Pd}(\mathrm{acac})_{2}$

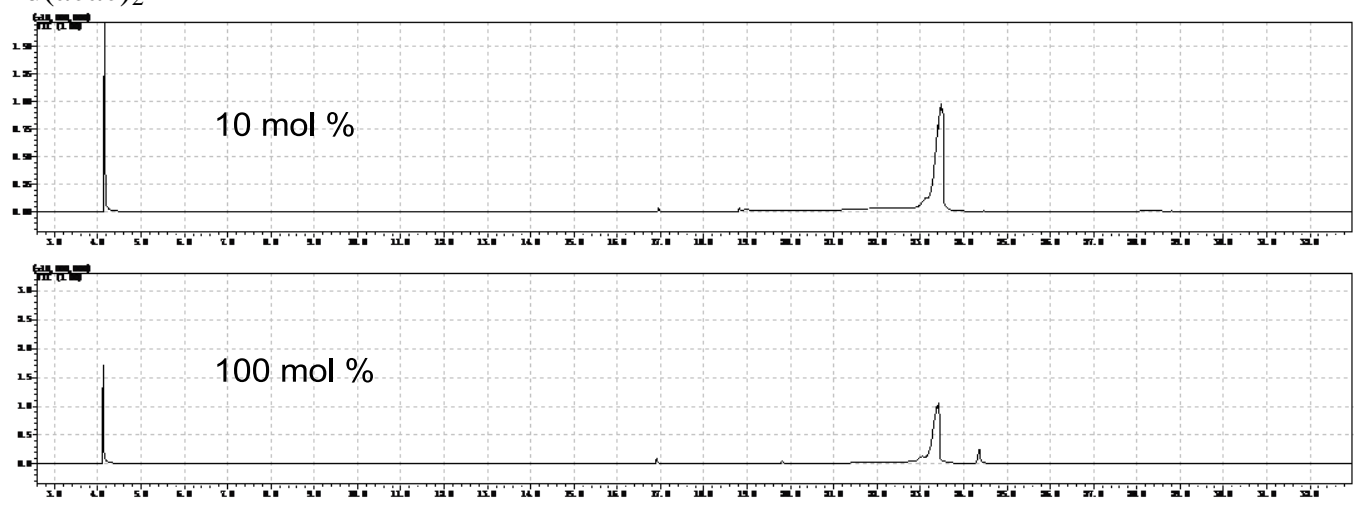

\section{$\operatorname{Pd}\left(\mathrm{PPh}_{3}\right)_{2} \mathrm{Cl}_{2}$}
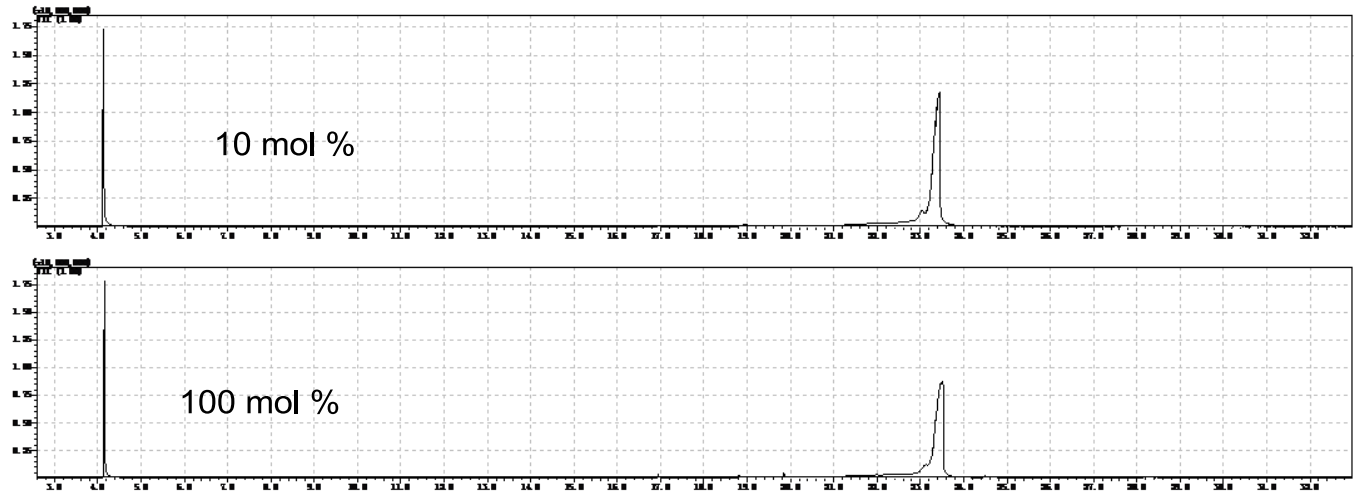

$\mathrm{IrCl}_{3}$
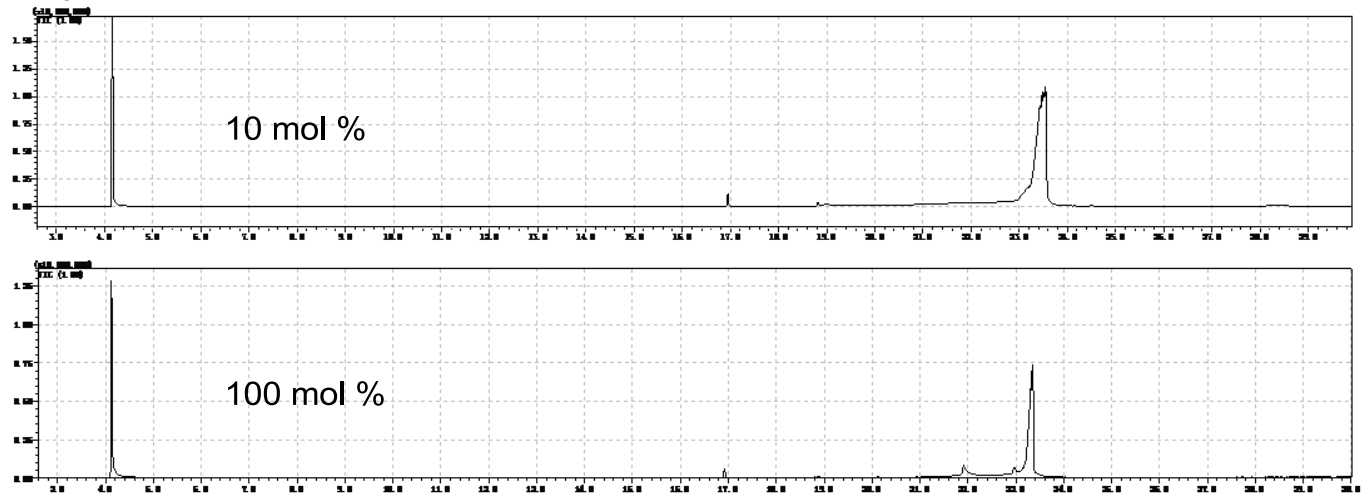

\section{$[\operatorname{Ir}(\mathrm{COD}) \mathrm{Cl}]_{2}$}

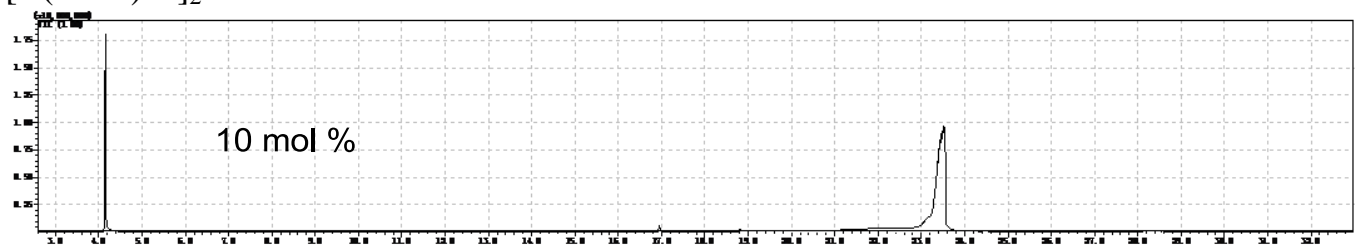




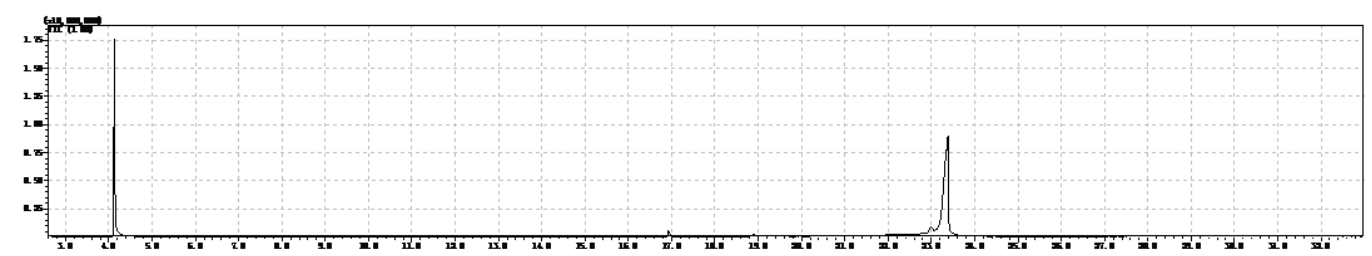

Scheme S9 Reactions of Hantzsch ester and bromobenzene in the presence of transition metal catalysts.

\section{Procedure for the synthesis of $8 p$}

A reaction tube was charged with the $f a c-\operatorname{Ir}(\mathrm{ppy})_{3}(0.65 \mathrm{mg}, 0.2 \mathrm{~mol} \%), \mathrm{Cs}_{2} \mathrm{CO}_{3}(325 \mathrm{mg}, 1.0 \mathrm{mmol}$, 1.0 equiv), $7 \mathbf{a}$ (921 mg, $3.0 \mathrm{mmol}, 3.0$ equiv), phenyl 2-bromo-2-methylpropanoate $\mathbf{1 q}(243 \mathrm{mg}, 1.0$ mmol, 1.0 equiv) and $\mathrm{CH}_{3} \mathrm{CN}(10 \mathrm{~mL})$. The mixture was stirred under irradiation of $12 \mathrm{~W}$ white LED strip under a nitrogen atmosphere at room temperature. The reaction was monitored by TLC. After the reaction completed, the solvent was removed under reduced pressure. The crude product was obtained from flash chromatography on silica gel $(\mathrm{PE} / \mathrm{EA}=98: 2, \mathrm{v} / \mathrm{v})$. The crude product was hydrolyzed with $\mathrm{NaOH}(2 \mathrm{M}, 0.4 \mathrm{~mL})$ in $\mathrm{C}_{2} \mathrm{H}_{5} \mathrm{OH}(1.6 \mathrm{~mL})$ at $80{ }^{\circ} \mathrm{C}$ for 5 hours and cooled to room temperature. Subsequently, $2 \mathrm{M} \mathrm{HCl}$ was added to the mixture until $\mathrm{pH}=3 \sim 4$ was reached. The organic layer was extracted with EtOAc $(3 \mathrm{X} 5 \mathrm{~mL})$. The combined organic layers were dried over $\mathrm{Na}_{2} \mathrm{SO}_{4}$ and concentrated under reduced pressure. Product 8 p (78.3 mg, 38\% two step, Scheme S10) was obtained as a white solid from chromatography on silica gel (PE/EA $=90: 10$, v/v). M.P. $165-167{ }^{\circ} \mathrm{C}$. IR (neat) 2976, 2877, 1683, 1471, 1457, 1290, 1173, 1141, 1096, 1075, $702 \mathrm{~cm}^{-1}$. ${ }^{1} \mathrm{H}$ NMR (400 MHz, CDCl3, TMS) $\delta 11.77(\mathrm{~s}, 1 \mathrm{H}), 7.39(\mathrm{~d}, J=7.6 \mathrm{~Hz}, 2 \mathrm{H}), 7.30(\mathrm{t}, J=7.6 \mathrm{~Hz}, 2 \mathrm{H}), 7.25-7.20(\mathrm{~m}, 1 \mathrm{H}), 1.50(\mathrm{~s}$, $6 \mathrm{H}), 1.11(\mathrm{~s}, 6 \mathrm{H}) .{ }^{13} \mathrm{C}$ NMR $(100 \mathrm{MHz}, \mathrm{CDCl} 3) \delta 183.5,145.4,127.9,127.3,126.1,48.7,42.3,25.0$, 22.0. HRMS (APCI): calcd. for $\mathrm{C}_{13} \mathrm{H}_{17} \mathrm{O}_{2}\left([\mathrm{M}-\mathrm{H}]^{-}\right)$: 205.1229, found: 205.1230 .

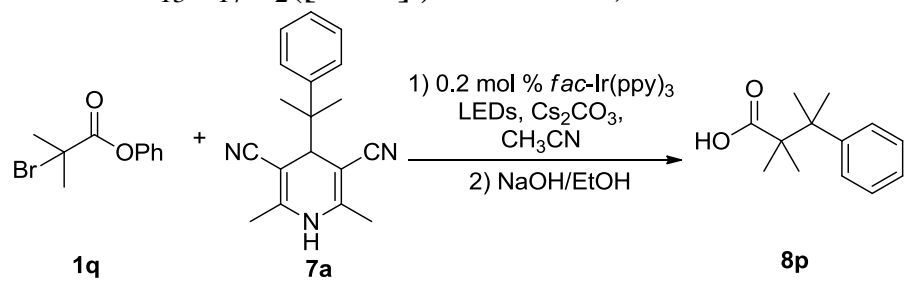

Scheme S10 Preparation of 8p.

\section{Procedure for synthesis of 9 and cultivation of single crystals}

A solution of $\mathbf{8 b}(88.8 \mathrm{mg}, 0.3 \mathrm{mmol})$ in $\mathrm{Et}_{2} \mathrm{O}(2 \mathrm{~mL})$ was added to a stirred suspension of $\mathrm{LiAlH}_{4}$ $(10.0 \mathrm{mg}, 0.26 \mathrm{mmol})$ on $\mathrm{Et}_{2} \mathrm{O}(1.0 \mathrm{~mL})$ at $0{ }^{\circ} \mathrm{C}$. The reaction was monitored by TLC until completion. Water and ethyl aceate were added and the layers were separated. The aqueous layer was extracted three times with EtOAc. The combined organic layers were washed with brine, dried $\left(\mathrm{MgSO}_{4}\right)$ and filtered. The filtrate was concentrated under reduced pressure. The crude product was dissolved in $\mathrm{CH}_{2} \mathrm{Cl}_{2}(10 \mathrm{~mL})$ and a solution of DMAP $(36.6 \mathrm{mg}, 0.3 \mathrm{mmol})$ and 3,5-dinitrobenzoyl chloride $(69 \mathrm{mg}$, $0.3 \mathrm{mmol})$ in $\mathrm{CH}_{2} \mathrm{Cl}_{2}(10 \mathrm{~mL})$ was added at room temperature under $\mathrm{N}_{2}$ atmosphere. The mixture was stirred overnight at room temperature. Then the reaction solution was diluted with $\mathrm{CH}_{2} \mathrm{Cl}_{2}$, washed with water, dried $\left(\mathrm{Na}_{2} \mathrm{SO}_{4}\right)$, and concentrated. The obtained residue was purified by chromatography 
on silica gel $(\mathrm{PE} / \mathrm{EA}=95: 5, \mathrm{v} / \mathrm{v})$ to give product $9(119.6 \mathrm{mg}, 81 \%$ two step, Scheme S11). A recrystallization of the product from a mixture solvent of dichloromethane and hexane at room temperature afforded crystals suitable for XRD analysis. M.P. 165-167 ${ }^{\circ} \mathrm{C}$. IR (neat) 3100, 2980, 2836, $1732,1628,1544,1512,1343,1275,743 \mathrm{~cm}^{-1} .{ }^{1} \mathrm{H}$ NMR (400 MHz, CDCl3, TMS) $\delta 9.16(\mathrm{t}, J=2.0$ $\mathrm{Hz}, 1 \mathrm{H}), 8.68(\mathrm{~d}, J=2.0 \mathrm{~Hz}, 2 \mathrm{H}), 7.36(\mathrm{~d}, J=8.7 \mathrm{~Hz}, 2 \mathrm{H}), 7.33-7.25(\mathrm{~m}, 5 \mathrm{H}), 6.53(\mathrm{~d}, J=8.7 \mathrm{~Hz}, 2 \mathrm{H})$, $6.12(\mathrm{~s}, 1 \mathrm{H}), 3.42(\mathrm{~s}, 3 \mathrm{H}), 1.57(\mathrm{~s}, 3 \mathrm{H}), 1.45(\mathrm{~s}, 3 \mathrm{H}), 1.44(\mathrm{~s}, 3 \mathrm{H}), 0.73(\mathrm{~s}, 3 \mathrm{H}) .{ }^{13} \mathrm{C}$ NMR $(100 \mathrm{MHz}$, $\mathrm{CDCl} 3) \delta 161.1,157.1,148.1,140.2,138.8,133.9,128.9,128.6,128.0,127.5,127.5,121.8,112.8$, $80.8,54.6,43.6,42.8,25.9,25.3,23.4,17.9$.

Scheme S11 Preparation of 9.

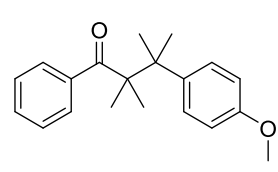

$8 b$

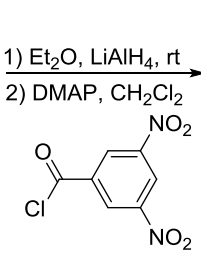

$\mathrm{NO}_{2}$

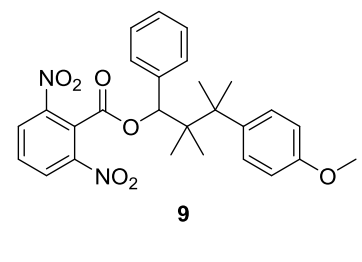

\section{Cyclic voltammetry experiments}

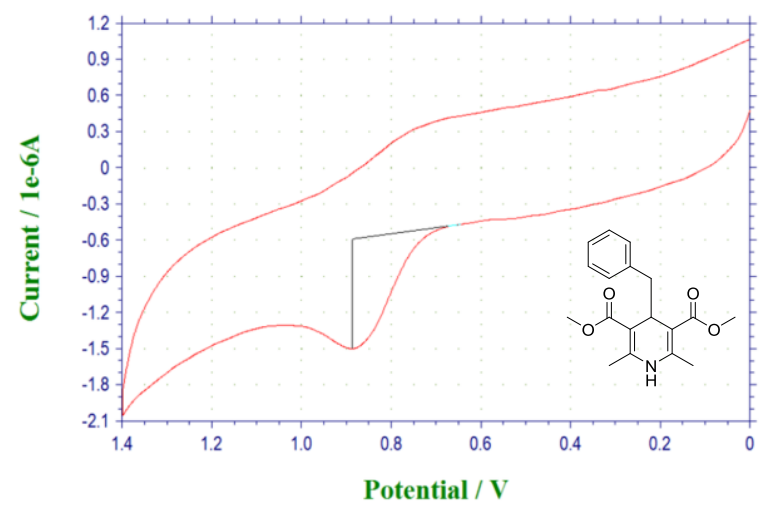

Cyclic voltammogram of Hantzsch ester $\mathbf{2 a}$ in $0.1 \mathrm{M}$ TBAP/ $\mathrm{CH}_{3} \mathrm{CN}$ at a Pt working electrode with a $\mathrm{Pt}$ counter electrode and a saturated calomel electrode (SCE). Ferrocene was used as an internal standard; potential sweep rate was $100 \mathrm{mV} / \mathrm{s}$. Ep=0.89 V.

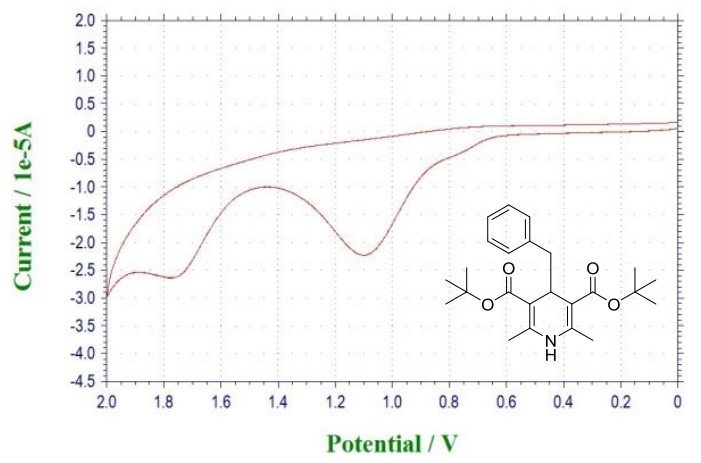

Cyclic voltammogram of Hantzsch ester $2 \mathbf{c}$ in $0.1 \mathrm{M}$ TBAP/ $\mathrm{CH}_{3} \mathrm{CN}$ at a Pt working electrode with a Pt counter electrode and a saturated calomel electrode (SCE). Ferrocene was used as an internal standard; potential sweep rate was $100 \mathrm{mV} / \mathrm{s}$. Ep=1.08 V. 


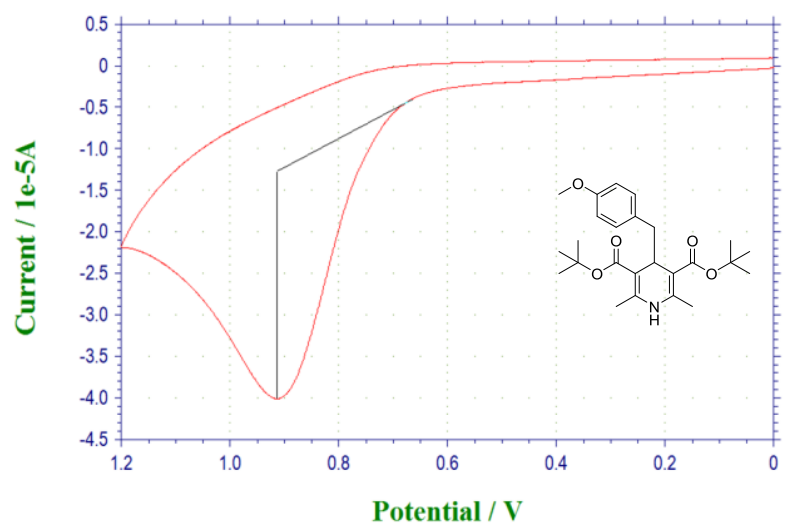

Cyclic voltammogram of Hantzsch ester $2 \mathbf{e}$ in $0.1 \mathrm{M} \mathrm{TBAP} / \mathrm{CH}_{3} \mathrm{CN}$ at a Pt working electrode with a Pt counter electrode and a saturated calomel electrode (SCE). Ferrocene was used as an internal standard; potential sweep rate was $100 \mathrm{mV} / \mathrm{s}$. Ep=0.91 V.

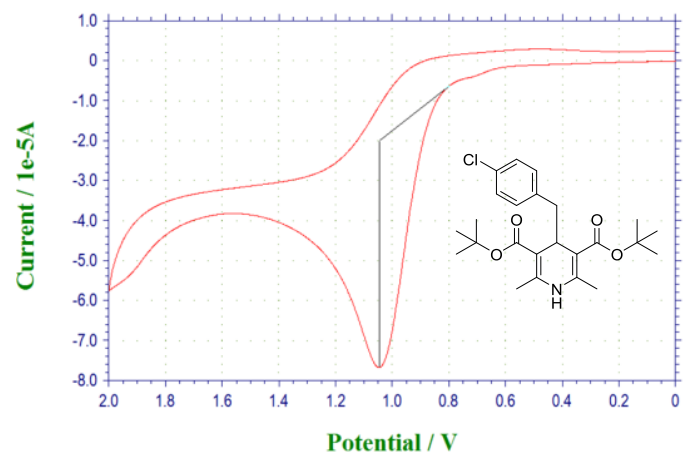

Cyclic voltammogram of Hantzsch ester $2 \mathbf{f}$ in $0.1 \mathrm{M}$ TBAP/ $\mathrm{CH}_{3} \mathrm{CN}$ at a Pt working electrode with a Pt counter electrode and a saturated calomel electrode (SCE). Ferrocene was used as an internal standard; potential sweep rate was $100 \mathrm{mV} / \mathrm{s}$. Ep=1.05 V.

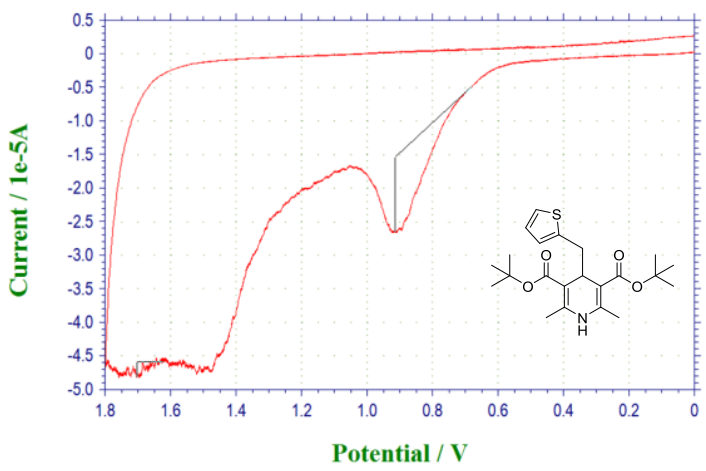

Cyclic voltammogram of Hantzsch ester $2 \mathrm{~g}$ in $0.1 \mathrm{M}$ TBAP/ $\mathrm{CH}_{3} \mathrm{CN}$ at a Pt working electrode with a $\mathrm{Pt}$ counter electrode and a saturated calomel electrode (SCE). Ferrocene was used as an internal standard; potential sweep rate was $100 \mathrm{mV} / \mathrm{s}$. Ep=0.92 V. 


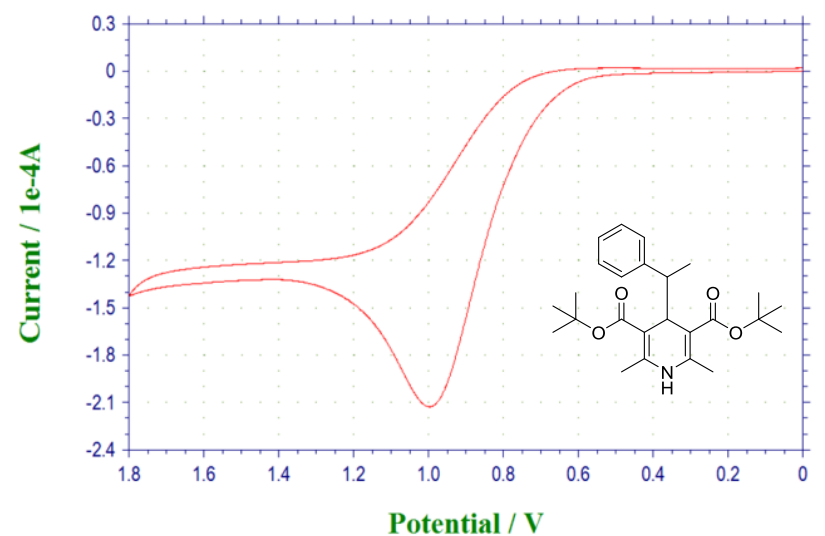

Cyclic voltammogram of Hantzsch ester $2 \mathbf{h}$ in $0.1 \mathrm{M} \mathrm{TBAP} / \mathrm{CH}_{3} \mathrm{CN}$ at a Pt working electrode with a $\mathrm{Pt}$ counter electrode and a saturated calomel electrode (SCE). Ferrocene was used as an internal standard; potential sweep rate was $100 \mathrm{mV} / \mathrm{s}$. Ep=0.98 V.

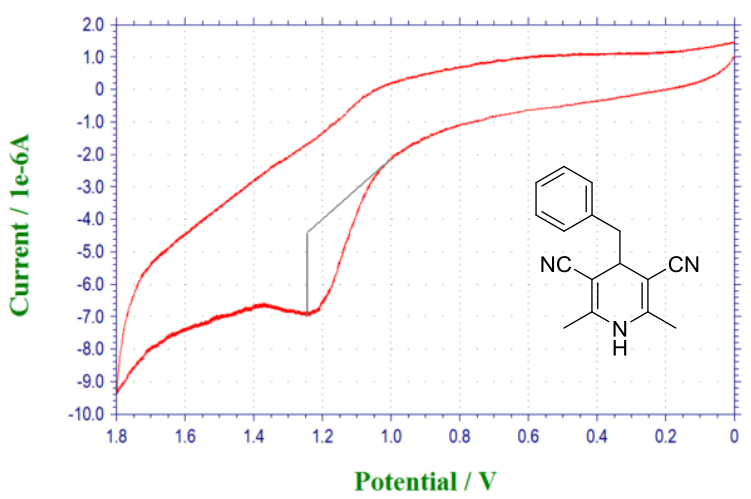

Cyclic voltammogram of $\mathbf{2 d}$ in $0.1 \mathrm{M} \mathrm{TBAP} / \mathrm{CH}_{3} \mathrm{CN}$ at a $\mathrm{Pt}$ working electrode with a $\mathrm{Pt}$ counter electrode and a saturated calomel electrode (SCE). Ferrocene was used as an internal standard; potential sweep rate was $100 \mathrm{mV} / \mathrm{s}$. Ep=1.25 V.

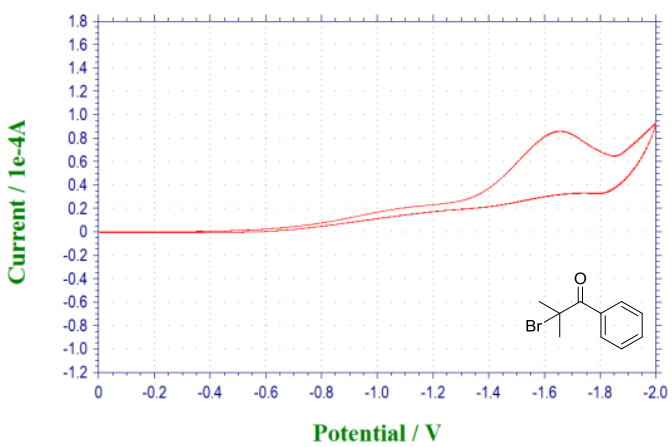

Cyclic voltammogram of 1a in $0.1 \mathrm{M} \mathrm{TBAP} / \mathrm{PhCN}$ at a $\mathrm{Pt}$ working electrode with a $\mathrm{Pt}$ counter electrode and a saturated calomel electrode (SCE). Ferrocene was used as an internal standard; potential sweep rate was $100 \mathrm{mV} / \mathrm{s}$. Ep= $-1.65 \mathrm{~V}$. 


\section{Procedure ${ }^{[14]}$ for syntheses of 2,6-dimethylpyridine-3,5-dicarboxylates and their electric}

\section{potential}

A reaction tube was charged with di-tert-butyl 2,6-dimethyl-1,4-dihydropyridine-3,5-dicarboxylate (154 mg, $0.5 \mathrm{mmol}, 1$ equiv) or $\mathbf{2 b}(172 \mathrm{mg}, 0.5 \mathrm{mmol}), \mathrm{SeO}_{2}(55 \mathrm{mg}, 0.5 \mathrm{mmol}, 1.0$ equiv) and $\mathrm{AcOH}$ $(2 \mathrm{~mL})$. The reaction was stirred at room temperature until completion monitored with TLC analysis. Solvent was removed under reduced pressure. Saturated aqueous solution of $\mathrm{NaHCO}_{3}$ and EtOAc were added and the layers were separated. The aqueous layer was extracted with EtOAc ( 3 X $5 \mathrm{~mL}$ ). The combined organic layers were washed with water, dried $\left(\mathrm{Na}_{2} \mathrm{SO}_{4}\right)$, and concentrated The corresponding product was obtained from chromatography on slical gel (for product from di-tert-butyl 2,6-dimethyl-1,4-dihydropyridine-3,5-dicarboxylate) or neutral $\mathrm{Al}_{2} \mathrm{O}_{3}$ (for product from $\mathbf{2 b}$ ). But $\mathbf{2 c}$ could not convert to the corresponding product using this mothed.<smiles>CCOC(=O)C1=C(C)NC(C)=C(C(=O)OCC)C1Cc1ccccc1</smiles><smiles>CCCCOC(=O)C1=C(C)NC(C)=C(C(=O)OCC(C)(C)C)C1</smiles>
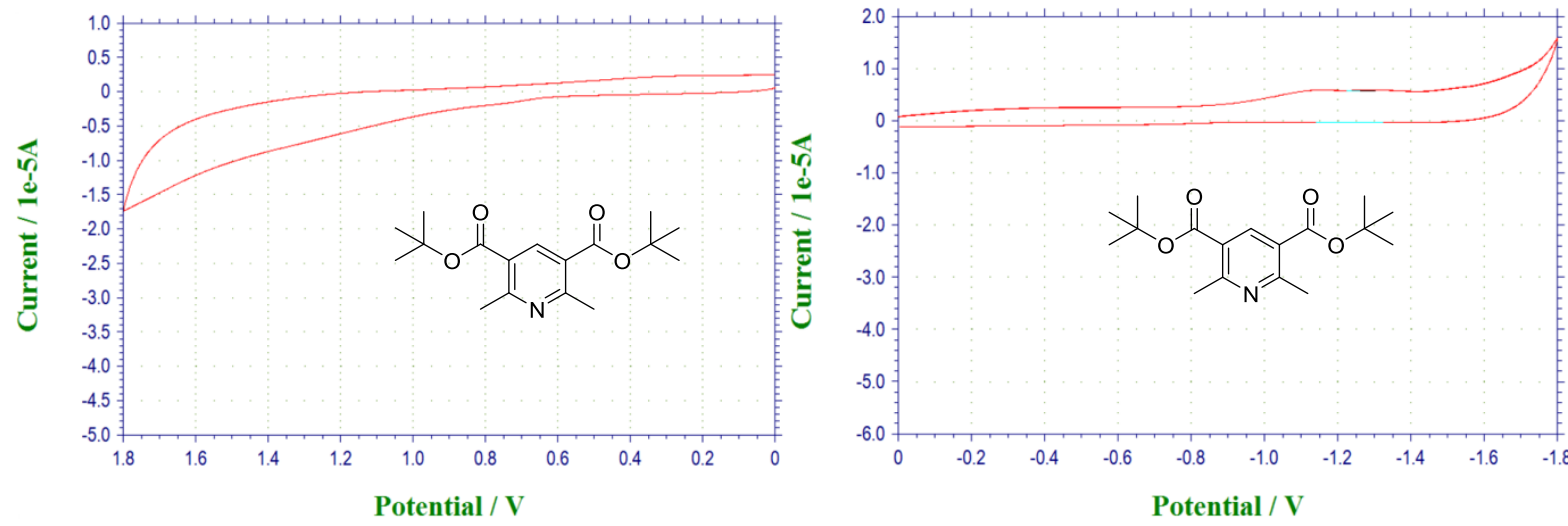

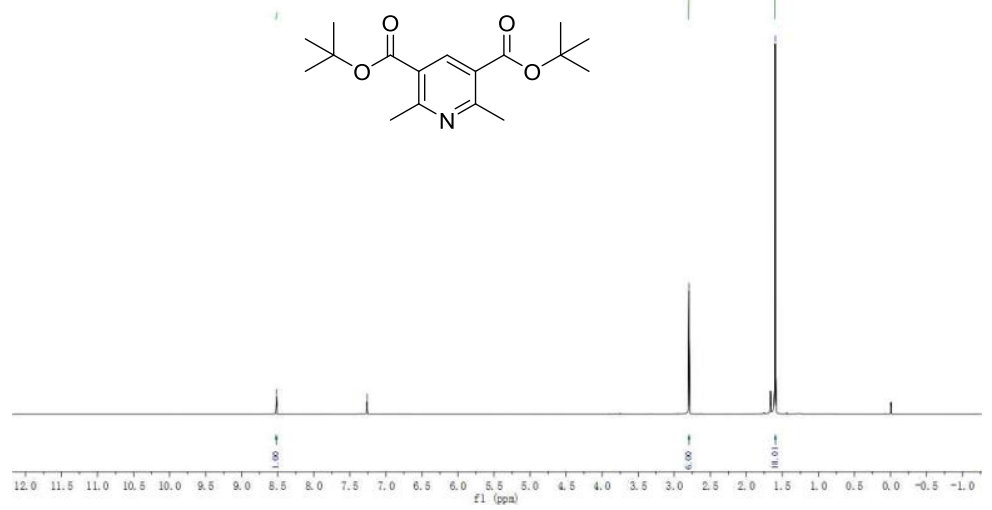

Cyclic voltammogram of di-tert-butyl 2,6-dimethylpyridine-3,5-dicarboxylate in $0.1 \mathrm{M} \mathrm{TBAP} / \mathrm{CH}_{3} \mathrm{CN}$ at a Pt working electrode with a Pt counter electrode and a saturated calomel electrode (SCE). Ferrocene was used as an internal standard; potential sweep rate was $100 \mathrm{mV} / \mathrm{s}$.
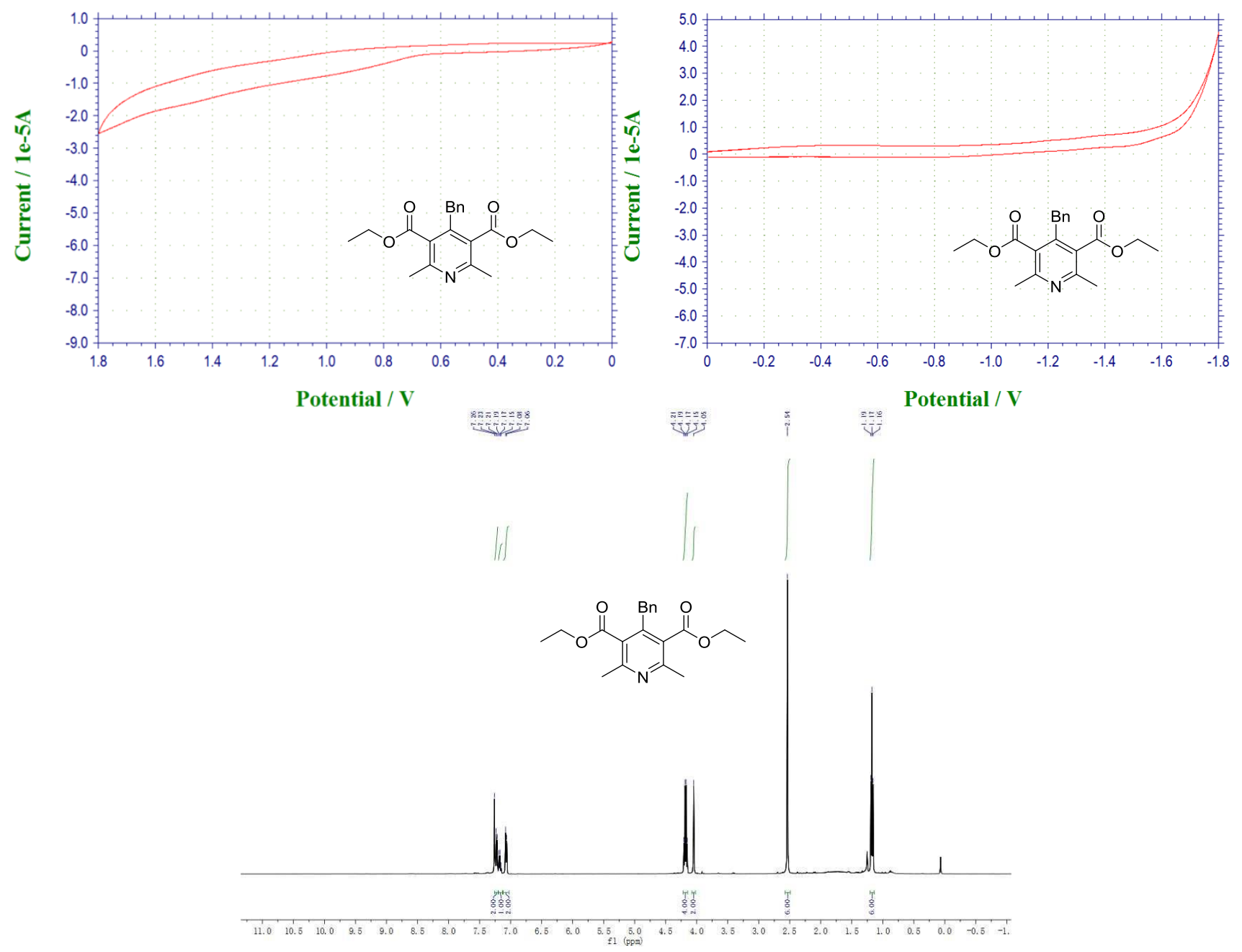

Cyclic voltammogram of diethyl 4-benzyl-2,6-dimethylpyridine-3,5-dicarboxylate in $0.1 \mathrm{M}$ $\mathrm{TBAP} / \mathrm{CH}_{3} \mathrm{CN}$ at a Pt working electrode with a Pt counter electrode and a saturated calomel electrode 
(SCE). Ferrocene was used as an internal standard; potential sweep rate was $100 \mathrm{mV} / \mathrm{s}$.

\section{Substrates did not convert to the corresponding product with $2 \mathrm{c}$}<smiles>CC(C)(Br)C(=O)C(C)(C)Br</smiles><smiles>CC(C)(Br)C(=O)c1cccc([N+](=O)[O-])c1</smiles><smiles>CN(C(=O)C(C)(C)Br)c1ccccc1</smiles><smiles>CC(C)(Br)C(=O)c1c2ccccc2cc2ccccc12</smiles>

\section{Syntheses and characterization of new compounds}

\section{2-Bromo-1-(3-fluorophenyl)-2-methylpropan-1-one (1e)}<smiles>CC(C)(Br)C(=O)c1cccc(F)c1</smiles>

With general procedure $\mathbf{A}$, reaction of 1-(3-fluorophenyl)-2-methylpropan-1-one $(1.0 \mathrm{mmol})$ and $\mathrm{PyHBr}_{3}(1.0 \mathrm{mmol})$ provided the $1 \mathrm{e}$ after flash column chromatography (1.0 vol \% EtOAc in petroleum ether) as a colorless oil (243 $\mathrm{mg}$, $99 \%) . \mathrm{R}_{f}=0.71$ (2.0 vol \% EtOAc/petroleum ether). IR (neat) 3006, 2972, 2921, 2864, 2843, 2822, 1744, 1679, 4630, 1054, 1032, $1012 \mathrm{~cm}^{-1} .{ }^{1} \mathrm{H}$ NMR $(400 \mathrm{MHz}$, $\left.\mathrm{CDCl}_{3}, \mathrm{TMS}\right) \delta$ 7.86-7.84 (m, 1H), 7.79-7.68 (m, 1H), 7.36-7.30 (m, 1H), 7.18-7.12 (m, 1H), $1.94(\mathrm{~s}$, $6 \mathrm{H}) .{ }^{13} \mathrm{C} \mathrm{NMR}\left(100 \mathrm{MHz}, \mathrm{CDCl}_{3}\right) \delta 195.6(\mathrm{~d}, J=2.1 \mathrm{~Hz}), 162.2(\mathrm{~d}, J=247.3 \mathrm{~Hz}), 136.8(\mathrm{~d}, J=6.6$ $\mathrm{Hz}), 129.8$ (d, $J=7.7 \mathrm{~Hz}), 125.8(\mathrm{~d}, J=3.1 \mathrm{~Hz}), 119.3(\mathrm{~d}, J=21.2 \mathrm{~Hz}), 117.0(\mathrm{~d}, J=23.4 \mathrm{~Hz}), 60.0$ (s), 31.3 (s). HRMS (EI): calcd. for $\mathrm{C}_{10} \mathrm{H}_{10} \mathrm{BrFO}\left([\mathrm{M}]^{+}\right)$: 243.9899 , found: 243.9904 .

\section{2-Bromo-1-(3-chlorophenyl)-2-methylpropan-1-one (1f)}<smiles>CC(C)(Br)C(=O)c1cccc(Cl)c1</smiles>

With general procedure $\mathbf{A}$, reaction of 1-(3-chlorophenyl)-2-methylpropan-1-one (1.0 mmol) and $\mathrm{PyHBr}_{3}(1.0 \mathrm{mmol})$ provided the $\mathbf{1 f}$ after flash column chromatography (1.0 vol \% EtOAc in petroleum ether) as a colorless oil (259 $\mathrm{mg}$, $99 \%) . \mathrm{R}_{f}=0.70$ (2.0 vol \% EtOAc/petroleum ether). IR (neat) 3004, 1980, 2916, $2865,1683,1567,1453,1275,1260,1055,1032,763,750 \mathrm{~cm}^{-1} .{ }^{1} \mathrm{H}$ NMR (400 $\mathrm{MHz}_{\mathrm{CDCl}}$, TMS) $\delta$ 7.99-7.98 (m, 1H), $7.95(\mathrm{~d}, J=7.9 \mathrm{~Hz}, 1 \mathrm{H}), 7.43-7.40(\mathrm{~m}, 1 \mathrm{H}), 7.29(\mathrm{t}, J=7.9$ $\mathrm{Hz}, 1 \mathrm{H}), 1.94(\mathrm{~s}, 6 \mathrm{H}) .{ }^{13} \mathrm{C} \mathrm{NMR}\left(100 \mathrm{MHz}, \mathrm{CDCl}_{3}\right) \delta 195.6,136.5,134.3,132.2,130.0,129.4,128.1$, 60.0, 31.3. HRMS (EI):calcd. for $\mathrm{C}_{10} \mathrm{H}_{10} \mathrm{BrClO}\left([\mathrm{M}]^{+}\right)$: 259.9604, found: 259.9605 . 


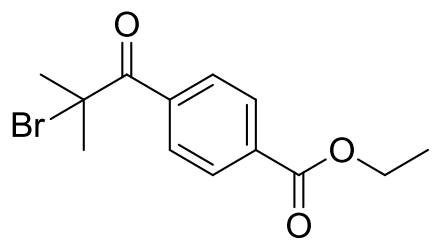

With general procedure A, reaction of ethyl 4-isobutyrylbenzoate (1.0 mmol) and $\mathrm{PyHBr}_{3}(1.0 \mathrm{mmol})$ provided the $\mathbf{1 h}$ after flash column chromatography (1.0 vol \% EtOAc in petroleum ether) as a colorless oil (299 $\mathrm{mg}, 99 \%)$. $\mathrm{R}_{f}=0.65$ (2.0 vol \% EtOAc in petroleum ether). IR (neat) 2980, 2934, 2920, 2843, 1748, 1714, 1679, 1555, 1454, 1275, 1054, 1032, $1012,750 \mathrm{~cm}^{-1} .{ }^{1} \mathrm{H}$ NMR $\left(400 \mathrm{MHz}, \mathrm{CDCl}_{3}, \mathrm{TMS}\right) \delta 8.07(\mathrm{~d}, J=8.5 \mathrm{~Hz}$, $2 \mathrm{H}), 8.02(\mathrm{~d}, J=8.5 \mathrm{~Hz}, 2 \mathrm{H}), 4.34(\mathrm{q}, J=7.1 \mathrm{~Hz}, 2 \mathrm{H}), 1.95(\mathrm{~s}, 6 \mathrm{H}), 1.34(\mathrm{t}, J=7.1 \mathrm{~Hz}, 3 \mathrm{H}) .{ }^{13} \mathrm{C}$ NMR $\left(100 \mathrm{MHz}, \mathrm{CDCl}_{3}\right) \delta 196.8,165.7,138.7,133.4,129.7,129.3,61.4,60.2,31.2,14.3$. HRMS (EI): calcd. for $\mathrm{C}_{13} \mathrm{H}_{15} \mathrm{BrO}_{3}\left([\mathrm{M}]^{+}\right)$: 298.0205, found: 298.0198 .

\section{1-(4-Amino-3,5-dibromophenyl)-2-bromo-2-methylpropan-1-one (1i)}<smiles>CC(C)(Br)C(=O)c1cc(Br)c(N)c(Br)c1</smiles>
With general procedure A

A, reaction of 1-(4-aminophenyl)-2-methylpropan-1-one (1.0 mmol) and $\mathrm{PyHBr}_{3}(3.0 \mathrm{mmol})$ provided the 1i after flash column chromatography (1.0 vol \% EtOAc in petroleum ether) as white solid (399 mg, 99\%), M.P. 105-107 ${ }^{\circ} \mathrm{C}, \mathrm{R}_{f}=0.50$ (2.0 vol \% EtOAc in petroleum ether). IR (neat) 3006, 2977, 2921, 2861, 2840, 1746, $1668,1606,1532,1489,1469,1316,1455,1275,1260,1054,1032,1010,765$, $746 \mathrm{~cm}^{-1} .{ }^{1} \mathrm{H}$ NMR $\left(400 \mathrm{MHz}, \mathrm{CDCl}_{3}, \mathrm{TMS}\right) \delta 8.31(\mathrm{~s}, 2 \mathrm{H}), 5.06(\mathrm{~s}, 2 \mathrm{H}), 2.01(\mathrm{~s}, 6 \mathrm{H}) .{ }^{13} \mathrm{C}$ NMR $(100$ $\left.\mathrm{MHz}, \quad \mathrm{CDCl}_{3}\right) \delta 192.3, \quad 145.6,134.6,125.0, \quad 107.1$, 60.1, 31.9. HRMS (EI):calcd. for $\mathrm{C}_{10} \mathrm{H}_{10} \mathrm{Br}_{3} \mathrm{ON}\left([\mathrm{M}]^{+}\right): 396.8312$, found: 396.8309 .

\section{2-Bromo-2-methyl-1-(naphthalen-2-yl)propan-1-one (1j)}

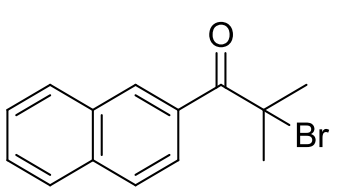

With general procedure

A, reaction of 2-methyl-1-(naphthalen-2-yl)propan-1-one $(1.0 \mathrm{mmol})$ and $\mathrm{PyHBr}_{3}(1.0 \mathrm{mmol})$ provided $\mathbf{1} \mathbf{j}$ after flash column chromatography (1.0 vol \% EtOAc in petroleum ether) as a colorless oil ( $277 \mathrm{mg}, 99 \%) \mathrm{R}_{f}=0.79(2.0 \mathrm{vol} \%$ EtOAc in petroleum ether). IR (neat) 3004, 2980, 2920, 2863, 1681, 1671, 1469, 1451, 1273, 1257, $1054,1032,1012,764,750 \mathrm{~cm}^{-1} .{ }^{1} \mathrm{H}$ NMR $\left(400 \mathrm{MHz}, \mathrm{CDCl}_{3}, \mathrm{TMS}\right) \delta 8.63(\mathrm{~s}, 1 \mathrm{H}), 8.04(\mathrm{dd}, J=8.7$, $1.7 \mathrm{~Hz}, 1 \mathrm{H}), 7.82(\mathrm{~d}, J=8.0 \mathrm{~Hz}, 1 \mathrm{H}), 7.72(\mathrm{~d}, J=8.7 \mathrm{~Hz}, 2 \mathrm{H}), 7.48-7.37(\mathrm{~m}, 2 \mathrm{H}), 1.97(\mathrm{~s}, 6 \mathrm{H}) .{ }^{13} \mathrm{C}$ NMR (100 MHz, $\left.\mathrm{CDCl}_{3}\right) \delta 196.6,134.9,132.1,132.0,131.5,129.5,128.3,127.7,127.6,126.6,125.9$, 60.5, 31.6. HRMS (EI):calcd. for $\mathrm{C}_{14} \mathrm{H}_{13} \mathrm{BrO}\left([\mathrm{M}]^{+}\right)$: 276.0150, found: 276.0152 .

\section{2-Bromo-2-methyl-1-(4-vinylphenyl)propan-1-one (1m)}

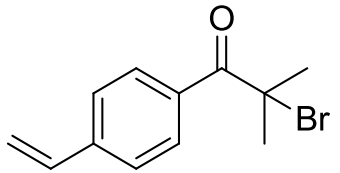

With general procedure $\mathbf{C}$, reaction of (4-vinylphenyl) magnesium bromide (1.0 $\mathrm{mmol}$ ) and 2-Bromo-2-methylpropionyl bromide $(1.0 \mathrm{mmol})$ provided $\mathbf{1 m}$ after flash column chromatography (1.0 vol \% EtOAc in petroleum ether) as a colorless oil ( $253 \mathrm{mg}, 99 \%) \mathrm{R}_{f}=0.75$ (2.0 vol \% EtOAc/petroleum ether). IR (neat) $2980,2920,2863,2844,1744,1679,1672,1602,1455,1259,1054,1032$,

$761,750 \mathrm{~cm}^{-1} .{ }^{1} \mathrm{H}$ NMR $\left(400 \mathrm{MHz}, \mathrm{CDCl}_{3}, \mathrm{TMS}\right) \delta 8.06(\mathrm{~d}, J=8.4 \mathrm{~Hz}, 2 \mathrm{H}), 7.39(\mathrm{~d}, J=8.4 \mathrm{~Hz}, 2 \mathrm{H})$, $6.68(\mathrm{dd}, J=17.6,10.9 \mathrm{~Hz}, 1 \mathrm{H}), 5.80(\mathrm{~d}, J=17.6 \mathrm{~Hz}, 1 \mathrm{H}), 5.32(\mathrm{~d}, J=10.9 \mathrm{~Hz}, 1 \mathrm{H}), 1.97(\mathrm{~s}, 6 \mathrm{H}) .{ }^{13} \mathrm{C}$ NMR $\left(100 \mathrm{MHz}, \mathrm{CDCl}_{3}\right) \delta 196.1,141.5,135.9,133.8,130.6,125.8,116.7,60.4,31.6 . \mathrm{HRMS}$ (EI):calcd. for $\mathrm{C}_{12} \mathrm{H}_{13} \mathrm{BrO}\left([\mathrm{M}]^{+}\right)$: 252.0150 , found: 252.0153 .

\section{2-Bromo-2-methyl-1-(thiophen-3-yl)propan-1-one (1p)}

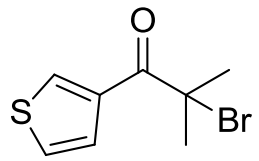

With general procedure $\mathbf{A}$, reaction of 2-methyl-1-(thiophen-2-yl)propan-1-one (1.0 mmol) and $\mathrm{PyHBr}_{3}(1.0 \mathrm{mmol})$ provided $\mathbf{1 p}$ after flash column chromatography $(1.0$ vol \% EtOAc in petroleum ether) as a colorless oil ( $231 \mathrm{mg}, 99 \%) . \mathrm{R}_{f}=0.68(2.0 \mathrm{vol} \%$ EtOAc in petroleum ether). IR (neat) 3002, 2981, 2922, 2863, 2842, 1712, 1681, 
$1555,1471,1275,1255,1054,1032,1006,764,750 \mathrm{~cm}^{-1} .{ }^{1} \mathrm{H}$ NMR $\left(400 \mathrm{MHz}, \mathrm{CDCl}_{3}, \mathrm{TMS}\right) \delta 8.31$ $(\mathrm{dd}, J=3.0,1.2 \mathrm{~Hz}, 1 \mathrm{H}), 7.65(\mathrm{dd}, J=5.1,1.2 \mathrm{~Hz}, 1 \mathrm{H}), 7.20(\mathrm{dd}, J=5.1,3.0 \mathrm{~Hz}, 1 \mathrm{H}), 1.93(\mathrm{~s}, 6 \mathrm{H})$. ${ }^{13} \mathrm{C}$ NMR $\left(100 \mathrm{MHz}, \mathrm{CDCl}_{3}\right) \delta 190.5,137.2,134.1,129.2,125.1,61.1,31.3$. HRMS (EI):calcd. for $\mathrm{C}_{8} \mathrm{H}_{9} \mathrm{BrOS}\left([\mathrm{M}]^{+}\right): 231.9557$, found: 231.9556 .

\section{4-Acetylphenyl 2-bromo-2-methylpropanoate (1r)}

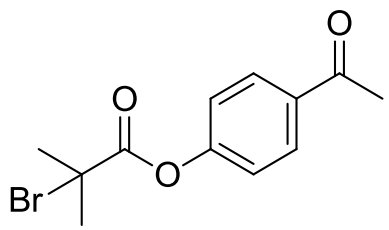

With general procedure $\mathbf{B}$, reaction of 1-(4-hydroxyphenyl) ethanone (1.0 $\mathrm{mmol})$ and 2-Bromo-2-methylpropionyl bromide $(1.0 \mathrm{mmol})$ provided $\mathbf{1 r}$ after flash column chromatography (1.0 vol \% EtOAc in petroleum ether) as white solid (285 mg, 99\%), M.P 65-67 ${ }^{\circ} \mathrm{C}, \mathrm{R}_{f}=0.68$ (2.0 vol \% EtOAc in petroleum ether). IR (neat) 2977, 2921, 2861, 1748, 1682, 1261, 1163, 1135, 1054, 1032, 1012, $750 \mathrm{~cm}^{-1}$. ${ }^{1} \mathrm{H}$ NMR (400 MHz, $\left.\mathrm{CDCl}_{3}, \mathrm{TMS}\right) \delta 7.89(\mathrm{~d}, J=$ $8.0 \mathrm{~Hz}, 2 \mathrm{H}), 7.12(\mathrm{~d}, J=8.0 \mathrm{~Hz}, 2 \mathrm{H}), 2.48(\mathrm{~s}, 3 \mathrm{H}), 1.96(\mathrm{~s}, 6 \mathrm{H}) .{ }^{13} \mathrm{C} \mathrm{NMR}\left(100 \mathrm{MHz}, \mathrm{CDCl}_{3}\right) \delta 196.6$, 169.4, 154.1, 134.7, 129.8, 121.1, 55.0, 30.3, 26.3. HRMS (EI):calcd. for $\mathrm{C}_{12} \mathrm{H}_{13} \mathrm{BrO}_{3}\left([\mathrm{M}]^{+}\right)$: 284.0048, found: 284.0042 .

\section{2-bromo-3-methoxy-2-methyl-1-phenylpropan-1-one $(1 \mathbf{x})^{[8]}$}<smiles>COCC(C)(Br)C(=O)c1ccccc1</smiles>

With general procedure A, reaction of 3-methoxy-2-methyl-1-phenylpropan-1-one $(10 \mathrm{mmol})$ and $\mathrm{PyHBr}_{3}(10 \mathrm{mmol})$ provided $\mathbf{1 x}$ after flash column chromatography (3 vol \% EtOAc in petroleum ether) as a colorless oil $(2.20 \mathrm{~g}, 81 \%) . \mathrm{R}_{f}=0.50$ (3.0 vol \%

EtOAc in petroleum ether). ${ }^{1} \mathrm{H} \mathrm{NMR}\left(400 \mathrm{MHz} \mathrm{CDCl}_{3}, \mathrm{TMS}\right) \delta 8.02-7.99(\mathrm{~m}, 2 \mathrm{H})$, $7.52-7.50(\mathrm{~m}, 1 \mathrm{H}), 7.44-7.40(\mathrm{~m}, 2 \mathrm{H}), 4.02(\mathrm{~d}, J=12.0 \mathrm{~Hz}, 1 \mathrm{H}), 3.80(\mathrm{~d}, J=12.0$

$\mathrm{Hz}, 1 \mathrm{H}), 3.31(\mathrm{~s}, 3 \mathrm{H}), 2.03(\mathrm{~s}, 3 \mathrm{H})$.

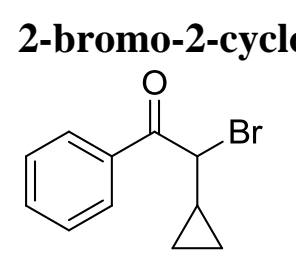

With general procedure $\mathbf{A}$, reaction of 2-cyclopropyl-1-phenylethanone (1.0 mmol) and $\mathrm{PyHBr}_{3}(1.0 \mathrm{mmol})$ provided $1 \mathbf{y}$ after flash column chromatography (1 vol \% EtOAc in petroleum ether) as a colorless oil $(190 \mathrm{mg}, 82 \%) . \mathrm{R}_{f}=0.50(1.0 \mathrm{vol} \%$ EtOAc in petroleum ether). ${ }^{1} \mathrm{H}$ NMR (400 MHz, $\left.\mathrm{CDCl}_{3}, \mathrm{TMS}\right) \delta 8.00$ (d, $J=7.6 \mathrm{~Hz}$, 2H), 7.61-7.57 (m, 1H), 7.52-7.46 (m, 2H), $4.47(\mathrm{~d}, J=10.2 \mathrm{~Hz}, 1 \mathrm{H}), 1.90-1.79(\mathrm{~m}, 1 \mathrm{H}), 0.94-0.88$ (m, $2 \mathrm{H}), 0.61-0.52(\mathrm{~m}, 1 \mathrm{H}), 0.51-0.42(\mathrm{~m}, 1 \mathrm{H})$.

\section{Di-tert-butyl-4-(4-methoxybenzyl)-2,6-dimethyl-1,4-dihydropyridine-3,5-dicarboxylate (2d)}

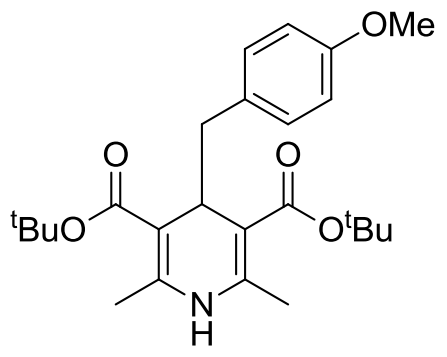

With general procedure $\mathbf{D}$, reaction of 4-methoxy-phenylacetaldehyde (10 mmol) and tert-butyl acetoacetate $(20 \mathrm{mmol})$ provided $\mathbf{2 d}$ after flash column chromatography (10 vol \% EtOAc in petroleum ether) as a white solid (2.6 g, 61\%). $\mathrm{R}_{f}=0.51$ (10 vol \% EtOAc in petroleum ether). M.P. $143-145^{\circ} \mathrm{C}$. IR (neat) $3347,2952,2878,1689,1681,1421,1275, \mathrm{~cm}^{-1} .{ }^{1} \mathrm{H}$ NMR (400 MHz, $\left.\mathrm{CDCl}_{3}, \mathrm{TMS}\right) \delta 6.93(\mathrm{~d}, J=8.5 \mathrm{~Hz}, 2 \mathrm{H}), 6.74(\mathrm{~d}, J=8.5$ $\mathrm{Hz}, 2 \mathrm{H}), 5.08(\mathrm{~s}, 1 \mathrm{H}), 4.09(\mathrm{t}, J=5.6 \mathrm{~Hz}, 1 \mathrm{H}), 3.76(\mathrm{~s}, 3 \mathrm{H}), 2.49(\mathrm{~d}, J=$ $5.6 \mathrm{~Hz}, 2 \mathrm{H}), 2.15(\mathrm{~s}, 6 \mathrm{H}), 1.43(\mathrm{~s}, 18 \mathrm{H}) .{ }^{13} \mathrm{C} \mathrm{NMR}\left(100 \mathrm{MHz}, \mathrm{CDCl}_{3}\right) \delta$ 167.2, 157.9, 144.2, 131.7, 131.0, 112.9, 103.3, 79.2, 55.2, 40.8, 36.0, 28.3, 19.2. HRMS (ESI): calcd. for $\mathrm{C}_{25} \mathrm{H}_{35} \mathrm{NNaO}_{5}\left([\mathrm{M}+\mathrm{Na}]^{+}\right): 452.2413$, found: 452.2405 .

\section{Di-tert-butyl-4-(4-chlorobenzyl)-2,6-dimethyl-1,4-dihydropyridine-3,5-dicarboxylate(2e)}




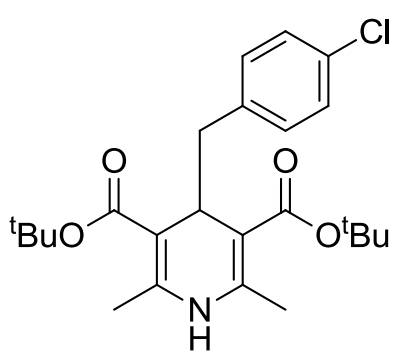

With general procedure $\mathbf{D}$, reaction of 2-(4-chlorophenyl)acetaldehyde (10 $\mathrm{mmol})$ and tert-butyl acetoacetate $(20 \mathrm{mmol})$ provided $\mathbf{2 e}$ after flash column chromatography (10 vol \% EtOAc in petroleum ether) as a white solid $(2.5 \mathrm{~g}$, $55 \%) . \mathrm{R}_{f}=0.60$ (10 vol \% EtOAc inpetroleum ether). M.P. $149-151{ }^{\circ} \mathrm{C} . \mathrm{IR}$ (neat) $3671,3644,3326,2987,2946,1746,1679,1644,1259,1275,1163$ $\mathrm{cm}^{-1},{ }^{1} \mathrm{H}$ NMR $\left(400 \mathrm{MHz}, \mathrm{CDCl}_{3}, \mathrm{TMS}\right) \delta 7.17(\mathrm{~d}, J=6.8 \mathrm{~Hz}, 2 \mathrm{H}), 6.97(\mathrm{~d}$, $J=6.8 \mathrm{~Hz}, 2 \mathrm{H}), 5.16(\mathrm{~s}, 1 \mathrm{H}), 4.12(\mathrm{t}, J=4.0 \mathrm{~Hz}, 1 \mathrm{H}), 2.54(\mathrm{~d}, J=4.0 \mathrm{~Hz}$, $2 \mathrm{H}), 2.17(\mathrm{~s}, 6 \mathrm{H}), 1.46(\mathrm{~s}, 18 \mathrm{H}) .{ }^{13} \mathrm{C} \mathrm{NMR}\left(100 \mathrm{MHz}, \mathrm{CDCl}_{3}\right) \delta 167.0,144.4$, 138.0, 131.5, 131.4, 127.4, 103.0, 79.5, 41.1, 35.9, 28.3, 19.3. HRMS (ESI): calcd. for $\mathrm{C}_{24} \mathrm{H}_{32} \mathrm{ClNNaO}_{4}\left([\mathrm{M}+\mathrm{Na}]^{+}\right): 456.1918$, found: 456.1918 .

\section{Di-tert-butyl-2,6-dimethyl-4-(thiophen-2-ylmethyl)-1,4-dihydropyridine-3,5-dicarboxylate(2f)}<smiles>CCCCCCOC(=O)C1=C(C)NC(C)=C(C(=O)OC(C)(C)C)C1Cc1cccs1</smiles>

With general procedure $\mathbf{D}$, reaction of 2-(thiophen-2-yl)acetaldehyde (10 $\mathrm{mmol})$ and tert-butyl acetoacetate $(20 \mathrm{mmol})$ provided $\mathbf{2 f}$ after flash column chromatography (15 vol \% EtOAc in petroleum ether) as a white solid (1.4 g, $35 \%) . \mathrm{R}_{f}=0.51\left(10 \mathrm{vol} \%\right.$ EtOAc in petroleum ether). M.P. $125-127^{\circ} \mathrm{C} . \mathrm{IR}$ (neat) $3736,3669,3642,2973,1747,1714,1275,1257 \mathrm{~cm}^{-1} .{ }^{1} \mathrm{H}$ NMR $(400$ $\left.\mathrm{MHz}, \mathrm{CDCl}_{3}, \mathrm{TMS}\right) \delta 7.09(\mathrm{~d}, J=5.1 \mathrm{~Hz}, 1 \mathrm{H}), 6.87-6.84(\mathrm{~m}, 1 \mathrm{H}), 6.63(\mathrm{~d}, J$ $=5.1 \mathrm{~Hz}, 1 \mathrm{H}), 5.20(\mathrm{~s}, 1 \mathrm{H}), 4.12(\mathrm{t}, J=5.4 \mathrm{~Hz}, 1 \mathrm{H}), 2.79(\mathrm{~d}, J=5.4 \mathrm{~Hz}, 2 \mathrm{H})$, $2.18(\mathrm{~s}, 6 \mathrm{H}), 1.45(\mathrm{~s}, 18 \mathrm{H}) .{ }^{13} \mathrm{C}$ NMR $\left(100 \mathrm{MHz}, \mathrm{CDCl}_{3}\right) \delta 167.0,144.6$, 141.7, 126.3, 126.0, 123.6, 103.0, 79.4, 36.2, 35.6, 28.3, 19.4. HRMS (ESI): calcd. for $\mathrm{C}_{22} \mathrm{H}_{31} \mathrm{NNaO}_{4} \mathrm{~S}\left([\mathrm{M}+\mathrm{Na}]^{+}\right): 428.1871$, found: 428.1872 .

\section{Di-tert-butyl-2,6-dimethyl-4-(1-phenylethyl)-1,4-dihydropyridine-3,5-dicarboxylate(2g)}<smiles>CCCCOC(=O)C1=C(C)NC(C)=C(C(=O)OC(C)(C)C)C1C(C)c1ccccc1</smiles>

With general procedure $\mathbf{D}$, reaction of 2-phenylpropanal $(10 \mathrm{mmol})$ and tert-butyl acetoacetate $(20 \mathrm{mmol})$ provided $\mathbf{2 g}$ after flash column chromatography (10 vol \% EtOAc in petroleum ether) as a white solid (1.6 g, $41 \%) . \mathrm{R}_{f}=0.53\left(10 \mathrm{vol} \%\right.$ EtOAc/petroleum ether). M.P. $138-140{ }^{\circ} \mathrm{C} . \mathrm{IR}$ (neat) $3303,2977,2907,1746,1677,1521,1422,1275,1260 \mathrm{~cm}^{-1},{ }^{1} \mathrm{H}$ NMR (400 MHz, CDCl 3 , TMS) $\delta$ 7.20-7.10 (m, 3H), 7.09-7.03 (m, 2H), $5.25(\mathrm{~s}$, $1 \mathrm{H}), 4.21(\mathrm{~d}, J=5.7 \mathrm{~Hz}, 1 \mathrm{H}), 2.76(\mathrm{dd}, J=7.2 \mathrm{~Hz}, J=5.7 \mathrm{~Hz}, 1 \mathrm{H}), 2.15(\mathrm{~s}$, $3 \mathrm{H}), 2.14(\mathrm{~s}, 3 \mathrm{H}), 1.46(\mathrm{~s}, 9 \mathrm{H}), 1.40(\mathrm{~s}, 9 \mathrm{H}), 1.18(\mathrm{~d}, J=7.2 \mathrm{~Hz}, 3 \mathrm{H}) .{ }^{13} \mathrm{C}$ NMR $\left(100 \mathrm{MHz}, \mathrm{CDCl}_{3}\right) \delta 167.7,167.6,144.3,144.0,143.9,128.6,127.1,125.7,102.6,102.2,79.2$, 19.2, 45.7, 40.3, 28.3, 28.2, 19.2, 19.1, 16.6. HRMS (ESI): calcd. for $\mathrm{C}_{25} \mathrm{H}_{35} \mathrm{NNaO}_{4}\left([\mathrm{M}+\mathrm{Na}]^{+}\right)$: 436.2464, found: 436.2477 .

\section{2,2-Dimethyl-1,3-diphenylpropan-1-one (3a)}

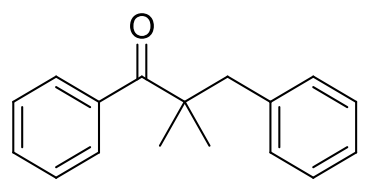

With general procedure $\mathbf{F}$, reaction of $\mathbf{1 a}(0.1 \mathrm{mmol})$ and $\mathbf{2 c}(0.3 \mathrm{mmol})$ provided the product 3a after flash column chromatography ( $0.5 \mathrm{vol} \%$ EtOAc in petroleum ether) as a colorless oil $(21.7 \mathrm{mg}, 91 \%) . \mathrm{R}_{f}=0.70(1.0 \mathrm{vol} \%$ EtOAc in petroleum ether). IR (neat) 3027, 2967, 1748, 1673, 1597, 1495, $1386,1261,959,747 \mathrm{~cm}^{-1} .{ }^{1} \mathrm{H}$ NMR $\left(400 \mathrm{MHz}, \mathrm{CDCl}_{3}, \mathrm{TMS}\right) \delta$ 7.42-7.44 (m, 2H), 7.38-7.34 (m, 1H), 7.31-7.27 (m, 2H), 7.19-7.12 (m, 3H), 7.06-7.00 (m, 2H), 3.00 (s, 2H), $1.22(\mathrm{~s}$, $6 \mathrm{H}) .{ }^{13} \mathrm{C}$ NMR $\left(100 \mathrm{MHz}, \mathrm{CDCl}_{3}\right) \delta 209.5,139.5,137.9,130.6,130.5,128.0,128.0,127.4,126.4,48.8$, 46.2, 26.1. HRMS (APCI): calcd. for $\mathrm{C}_{17} \mathrm{H}_{19} \mathrm{O}\left([\mathrm{M}+\mathrm{H}]^{+}\right)$: 239.1436, found: 239.1434 . 


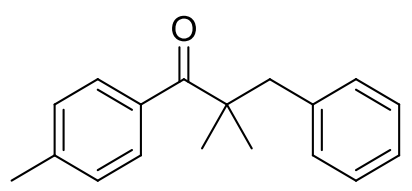

With general procedure $\mathbf{F}$, reaction of $\mathbf{1 b}(0.1 \mathrm{mmol})$ and $\mathbf{2 c}(0.3 \mathrm{mmol})$ provided the product $\mathbf{3 b}$ after flash column chromatography $(0.5 \mathrm{vol} \%$ EtOAc in petroleum ether) as a colorless oil $(20.4 \mathrm{mg},, 81 \%) . \mathrm{R}_{f}=0.71(1.0$ vol \% EtOAc in petroleum ether). IR (neat) 3061, 3027, 2967, 1926, 1673, $1506,1386,1261,1072,747,700 \mathrm{~cm}^{-1} .{ }^{1} \mathrm{H}$ NMR $\left(400 \mathrm{MHz}, \mathrm{CDCl}_{3}\right) \delta 7.44$ (d, $J=8.0 \mathrm{~Hz}, 2 \mathrm{H}), 7.16-7.09$ (m, 5H), $7.01(\mathrm{~d}, J=8.0 \mathrm{~Hz}, 2 \mathrm{H}), 3.01$ (s, 2H), 2.30 (s, 3H), $1.23(\mathrm{~s}, 6 \mathrm{H})$. ${ }^{13} \mathrm{C}$ NMR $\left(100 \mathrm{MHz}, \mathrm{CDCl}_{3}\right) \delta 208.5141 .2,138.0,136.3,130.5,128.7,128.0,128.0,126.3,48.7,46.4$, 26.2, 21.4. HRMS (APCI): calcd. for $\mathrm{C}_{18} \mathrm{H}_{21} \mathrm{O}\left([\mathrm{M}+\mathrm{H}]^{+}\right): 253.1592$, found: 253.1592 .

\section{1-(4-Methoxyphenyl)-2,2-dimethyl-3-phenylpropan-1-one (3c)}

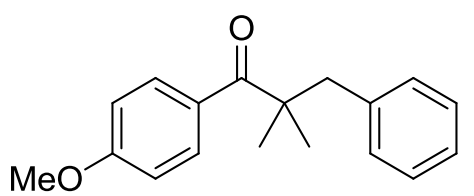

With general procedure $\mathbf{F}$, reaction of $\mathbf{1 c}(0.1 \mathrm{mmol})$ and $\mathbf{2 c}(0.3 \mathrm{mmol})$ provided the product $3 \mathbf{c}$ after flash column chromatography $(0.5 \mathrm{vol} \%$ EtOAc in petroleum ether) as a colorless oil $(21.4 \mathrm{mg}, 80 \%) . \mathrm{R}_{f}=0.71$ (1.0 vol \% EtOAc in petroleum ether). IR (neat), 3062, 3027, 1668, 1601, $1507,1275,1260,1054,1032,750,703 \mathrm{c} \mathrm{m}^{-1} .{ }^{1} \mathrm{H}$ NMR $(400 \mathrm{MHz}$, $\left.\mathrm{CDCl}_{3}, \mathrm{TMS}\right) \delta 7.66(\mathrm{~d}, J=8.0 \mathrm{~Hz}, 2 \mathrm{H}), 7.14(\mathrm{t}, J=8.0 \mathrm{~Hz}, 3 \mathrm{H}), 7.00(\mathrm{~d}, J=8.0 \mathrm{~Hz}, 2 \mathrm{H}), 6.82(\mathrm{~d}, J=$ $8.7 \mathrm{~Hz}, 2 \mathrm{H}), 3.78(\mathrm{~s}, 3 \mathrm{H}), 3.03(\mathrm{~s}, 2 \mathrm{H}), 1.25(\mathrm{~s}, 6 \mathrm{H}) .{ }^{13} \mathrm{C} \mathrm{NMR}\left(100 \mathrm{MHz}, \mathrm{CDCl}_{3}\right) \delta 206.3,161.9$, 137.1, 131.1, 130.7, 130.4, 128.0, 126.3, 113.3, 55.4, 48.6, 46.6, 26.3 HRMS (APCI): calcd. for $\mathrm{C}_{18} \mathrm{H}_{21} \mathrm{O}_{2}\left([\mathrm{M}+\mathrm{H}]^{+}\right): 269.1542$, found: 269.1542 .

\section{1-(4-Fluorophenyl)-2,2-dimethyl-3-phenylpropan-1-one (3d)}

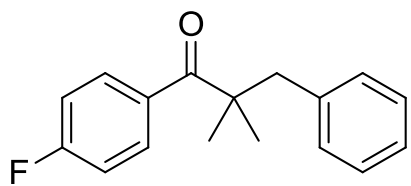

With general procedure $\mathbf{F}$, reaction of $\mathbf{1 d}(0.1 \mathrm{mmol})$ and $\mathbf{2 c}(0.3 \mathrm{mmol})$ provided the product $\mathbf{3 d}$ after flash column chromatography $(0.5 \mathrm{vol} \%$ EtOAc in petroleum ether) as a colorless oil $(21.8 \mathrm{mg}, 85 \%) . \mathrm{R}_{f}=0.70(1.0$ vol \% EtOAc in petroleum ether). IR (neat), 3052, 1981, 1672, 1599, 1275, $1260,1157,1054,1032,1012,764,749 \mathrm{~cm}^{-1}$. ${ }^{1} \mathrm{H}$ NMR $\left(400 \mathrm{MHz}, \mathrm{CDCl}_{3}\right.$, TMS) $\delta$ 7.60-7.57 (m, 5.5 Hz, 2H), 7.26-7.21 (m, 3H), 7.09-7.03 (m, 4H), 3.07 (s, 2H), $1.31(\mathrm{~s}, 6 \mathrm{H})$. ${ }^{13} \mathrm{C}$ NMR $\left(100 \mathrm{MHz}, \mathrm{CDCl}_{3}\right) \delta 207.5,164.1(\mathrm{~d}, J=251 \mathrm{~Hz}), 137.7,135.2(\mathrm{~d}, J=3 \mathrm{~Hz}), 130.4,130.3$ $(\mathrm{d}, J=9 \mathrm{~Hz}), 128.1,126.5,115.1(\mathrm{~d}, J=21 \mathrm{~Hz}), 48.8,46.4,26.1$. HRMS (APCI): calcd. for $\mathrm{C}_{17} \mathrm{H}_{18} \mathrm{FO}\left([\mathrm{M}+\mathrm{H}]^{+}\right): 257.1342$, found: 257.1349 .

\section{1-(3-Fluorophenyl)-2,2-dimethyl-3-phenylpropan-1-one (3e)}

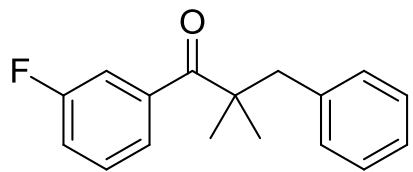

With general procedure $\mathbf{F}$, reaction of $1 \mathbf{e}(0.1 \mathrm{mmol})$ and $\mathbf{2 c}(0.3 \mathrm{mmol})$ provided the product $3 \mathrm{e}$ after flash column chromatography $(0.5$ vol $\%$ EtOAc in petroleum ether) as a colorless oil $(17.9 \mathrm{mg}, 70 \%) . \mathrm{R}_{f}=0.72(1.0$ vol \% EtOAc in petroleum ether). IR (neat), 3000, 2926, 1682, 1457, 1275, $1054,1032,1014,880,750 \mathrm{~cm}^{-1} .{ }^{1} \mathrm{H}$ NMR $\left(400 \mathrm{MHz}, \mathrm{CDCl}_{3}, \mathrm{TMS}\right) \delta$

7.36-7.31 (m, 1H), 7.27-7.20 (m, 4H), 7.15-7.08 (m, 4H), $3.05(\mathrm{~s}, 2 \mathrm{H}), 1.29(\mathrm{~s}, 6 \mathrm{H}) .{ }^{13} \mathrm{C}$ NMR $(100$ $\left.\mathrm{MHz}, \mathrm{CDCl}_{3}\right) \delta 208.3,162.2(\mathrm{~d}, J=246 \mathrm{~Hz}), 141.4(\mathrm{~d}, J=6 \mathrm{~Hz}), 137.6,130.5,129.7(\mathrm{~d}, J=7 \mathrm{~Hz})$, 128.1, 126.5, $123.0(\mathrm{~d}, J=3 \mathrm{~Hz}), 117.6(\mathrm{~d}, J=21 \mathrm{~Hz}), 114.6(\mathrm{~d}, J=23 \mathrm{~Hz}), 48.9,46.2$, 26.0. HRMS (APCI): calcd. for $\mathrm{C}_{17} \mathrm{H}_{18} \mathrm{FO}\left([\mathrm{M}+\mathrm{H}]^{+}\right)$: 257.1342, found: 257.1340 .

\section{1-(3-Chlorophenyl)-2,2-dimethyl-3-phenylpropan-1-one (3f)}<smiles>CC(C)(Cc1ccccc1)C(=O)c1cccc(Cl)c1</smiles>

With general procedure $\mathbf{F}$, reaction of $\mathbf{1 f}(0.1 \mathrm{mmol})$ and $\mathbf{2 c}(0.3 \mathrm{mmol})$ provided the product $3 \mathbf{f}$ after flash column chromatography $(0.5$ vol $\%$ 
EtOAc in petroleum ether) as a colorless oil $(23.1 \mathrm{mg}, 85 \%) . \mathrm{R}_{f}=0.70$ (1.0 vol \% EtOAc in petroleum ether). IR (neat), 2980, 2845, 1681, 1557, 1453, 1340, 1275, 1054, 1032, 1015, 764, $749 \mathrm{~cm}^{-1} .{ }^{1} \mathrm{H}$ NMR (400 MHz, $\mathrm{CDCl}_{3}$, TMS) $\delta$ 7.42-7.40 (m, 2H), 7.32-7.23 (m, 5H), 7.13-7.09 (m, 2H), $3.05(\mathrm{~s}$, 2H), $1.30(\mathrm{~s}, 6 \mathrm{H}) . .{ }^{13} \mathrm{C} \mathrm{NMR}\left(100 \mathrm{MHz}, \mathrm{CDCl}_{3}\right) \delta 208.4,141.1,137.6,134.2,130.5,130.5$ 129.4, 128.1, 127.5, 126.6, 125.3, 48.9, 46.2, 26.0. HRMS (APCI): calcd. for $\mathrm{C}_{17} \mathrm{H}_{18} \mathrm{ClO}\left([\mathrm{M}+\mathrm{H}]^{+}\right)$: 273.1046, found: 273.1045.

\section{1-Mesityl-2,2-dimethyl-3-phenylpropan-1-one (3g)}

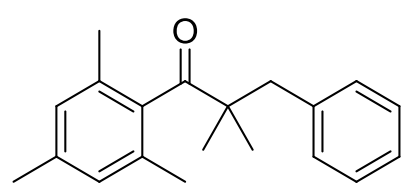

With general procedure $\mathbf{F}$, reaction of $\mathbf{1 g}(0.1 \mathrm{mmol})$ and $\mathbf{2 c}(0.3 \mathrm{mmol})$ provided the product $3 \mathrm{~g}$ after flash column chromatography $(0.5$ vol \% EtOAc in petroleum ether) as a colorless oil $(20.1 \mathrm{mg}, 74 \%) . \mathrm{R}_{f}=0.71(1.0$ vol \% EtOAc in petroleum ether). IR (neat), 2981, 2920, 2861, 2844, 1661, 1496, 1275, 1260, 1054, 1032, 1009, 763, 749, $702 \mathrm{~cm}^{-1} .{ }^{1} \mathrm{H}$ NMR (400 $\mathrm{MHz}_{\mathrm{CDCl}}$, TMS) $\delta$ 7.26-7.21 (m, 3H), $7.17(\mathrm{~d}, J=7.0 \mathrm{~Hz}, 2 \mathrm{H}), 6.83(\mathrm{~s}, 2 \mathrm{H}), 2.95(\mathrm{~s}, 2 \mathrm{H}), 2.27$ (s, $3 \mathrm{H}), 2.16(\mathrm{~s}, 6 \mathrm{H}), 1.12(\mathrm{~s}, 6 \mathrm{H}) .{ }^{13} \mathrm{C}$ NMR $\left(100 \mathrm{MHz}, \mathrm{CDCl}_{3}\right) \delta 218.8,139.3,137.5,137.5,132.3,131.2$, 128.5, 127.8, 126.3, 49.0, 44.9, 24.7, 20.9, 20.4. HRMS (EI): calcd. for $\mathrm{C}_{20} \mathrm{H}_{23} \mathrm{O}\left([\mathrm{M}-\mathrm{H}]^{+}\right): 279.1749$, found: 279.1752 .

\section{Ethyl 4-(2,2-dimethyl-3-phenylpropanoyl)benzoate (3h)}

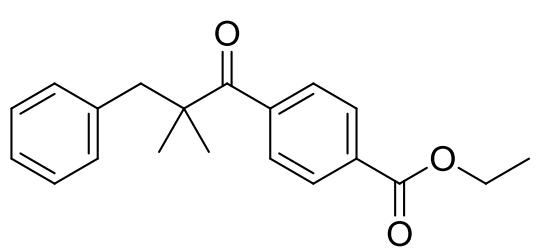

With general procedure $\mathbf{F}$, reaction of $\mathbf{1 h}(0.1 \mathrm{mmol})$ and $\mathbf{2 c}(0.3$ mmol) provided the product $\mathbf{3 h}$ after flash column chromatography (0.5 vol \% EtOAc in petroleum ether) as a colorless oil $(24.5 \mathrm{mg}$, $79 \%) . \mathrm{R}_{f}=0.60$ (1.0 vol \% EtOAc in petroleum ether). IR (neat), 2982, 2778, 1716, 1681, 1557, 1275, 1105, 1032, 1015, 749, 703 $\mathrm{cm}^{-1} .{ }^{1} \mathrm{H}$ NMR $\left(400 \mathrm{MHz}, \mathrm{CDCl}_{3}, \mathrm{TMS}\right) \delta 8.02(\mathrm{~d}, J=8.3 \mathrm{~Hz}, 2 \mathrm{H})$, $7.42(\mathrm{~d}, J=8.3 \mathrm{~Hz}, 2 \mathrm{H}), 7.28-7.22(\mathrm{~m}, 3 \mathrm{H}), 7.10(\mathrm{~d}, J=6.8 \mathrm{~Hz}, 2 \mathrm{H}), 4.38(\mathrm{q}, J=7.1 \mathrm{~Hz}, 2 \mathrm{H}), 3.04(\mathrm{~s}$, $2 \mathrm{H}), 1.40(\mathrm{t}, J=7.1 \mathrm{~Hz}, 3 \mathrm{H}), 1.28(\mathrm{~s}, 6 \mathrm{H}) .{ }^{13} \mathrm{C} \mathrm{NMR}\left(100 \mathrm{MHz}, \mathrm{CDCl}_{3}\right) \delta 209.9,165.8,143.6,137.6$, 131.9, 130.5, 129.3, 128.1, 126.9, 126.6, 61.3, 48.9, 46.1, 25.9, 14.3. HRMS (APCI): calcd. for $\mathrm{C}_{20} \mathrm{H}_{23} \mathrm{O}_{3}\left([\mathrm{M}+\mathrm{H}]^{+}\right): 311.1647$, found: 311.1645 .

\section{1-(4-Amino-3,5-dibromophenyl)-2,2-dimethyl-3-phenylpropan-1-one (3i)}

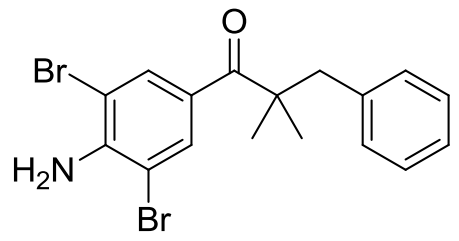

With general procedure $\mathbf{F}$, reaction of $\mathbf{1 i}(0.1 \mathrm{mmol})$ and $\mathbf{2 c}(0.3 \mathrm{mmol})$ provided the product $3 \mathbf{i}$ after flash column chromatography $(0.5$ vol \% EtOAc in petroleum ether) as white solid (32.9 mg, 80\%). $\mathrm{R}_{f}=0.58(1.0$ vol \% EtOAc in petroleum ether). IR (neat), 2967, 1914, 1840, 1714, $1669,1606,1275,1054,1032,763,749 \mathrm{~cm}^{-1}$. ${ }^{1} \mathrm{H}$ NMR $\left(400 \mathrm{MHz}, \mathrm{CDCl}_{3}\right.$, TMS) $\delta 7.80(\mathrm{~s}, 2 \mathrm{H}), 7.26-7.19(\mathrm{~m}, 3 \mathrm{H}), 7.07-7.05(\mathrm{~m}, 2 \mathrm{H}), 4.92(\mathrm{~s}, 2 \mathrm{H})$,

$3.06(\mathrm{~s}, 2 \mathrm{H}), 1.32(\mathrm{~s}, 6 \mathrm{H}) . .{ }^{13} \mathrm{C} \mathrm{NMR}\left(100 \mathrm{MHz}, \mathrm{CDCl}_{3}\right) \delta 203.2,144.6,137.7,132.9,130.3,129.1$, 128.1, 126.6, 107.4, 48.7, 46.8, 26.5. HRMS (APCI): calcd. for $\mathrm{C}_{17} \mathrm{H}_{17} \mathrm{Br}_{2} \mathrm{NO}\left([\mathrm{M}+\mathrm{H}]^{+}\right)$: 409.9755, found: 409.9748 .

\section{2,2-Dimethyl-1-(naphthalen-2-yl)-3-phenylpropan-1-one (3j)}

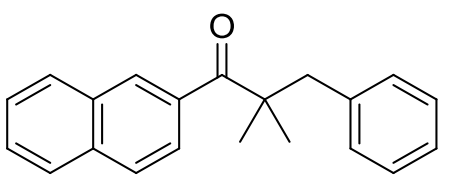

With general procedure $\mathbf{F}$, reaction of $\mathbf{1 j}(0.1 \mathrm{mmol})$ and $\mathbf{2 c}(0.3 \mathrm{mmol})$ provided the product $\mathbf{3 j}$ after flash column chromatography $(0.5$ vol \% EtOAc in petroleum ether) as a colorless oil $(23.9 \mathrm{mg}, 83 \%) . \mathrm{R}_{f}=0.75$ (1.0 vol \% EtOAc in petroleum ether). IR (neat), 2987, 1715, 1668, 1644, $1275,1055,1032,1015,765,749,702 \mathrm{~cm}^{-1}$. ${ }^{1} \mathrm{H}$ NMR $\left(400 \mathrm{MHz}, \mathrm{CDCl}_{3}\right.$, 
TMS) $\delta 7.98(\mathrm{~s}, 1 \mathrm{H}), 7.84(\mathrm{t}, J=8.2 \mathrm{~Hz}, 3 \mathrm{H}), 7.62-7.51(\mathrm{~m}, 3 \mathrm{H}), 7.28-7.20(\mathrm{~m}, 3 \mathrm{H}), 7.16-7.11(\mathrm{~m}, 2 \mathrm{H})$, $3.15(\mathrm{~s}, 2 \mathrm{H}), 1.37(\mathrm{~s}, 6 \mathrm{H}) . .{ }^{13} \mathrm{C}$ NMR $\left(100 \mathrm{MHz}, \mathrm{CDCl}_{3}\right) \delta 209.3,137.9,136.7,134.1,132.3,130.5$, 129.1, 128.1, 128.0, 127.8, 127.6, 127.6, 126.6, 126.5, 124.6, 49.0, 46.6, 26.3. HRMS (APCI): calcd. for $\mathrm{C}_{21} \mathrm{H}_{21} \mathrm{O}\left([\mathrm{M}+\mathrm{H}]^{+}\right)$: 289.1592, found: 289.1591 .

\section{(1-Benzylcyclopentyl)(phenyl)methanone (3k)}

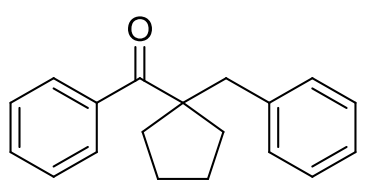

With general procedure $\mathbf{F}$, reaction of $\mathbf{1 k}(0.1 \mathrm{mmol})$ and $\mathbf{2 c}(0.3 \mathrm{mmol})$ provided the product $3 \mathbf{k}$ after flash column chromatography $(0.5$ vol \% EtOAc in petroleum ether) as a colorless oil $(19.8 \mathrm{mg}, 75 \%) . \mathrm{R}_{f}=0.70(1.0 \mathrm{vol} \%$ EtOAc in petroleum ether). IR (neat), 2981, 2920, 1672, 1454, 1275, 1260, $1054,1032,1013,764,750,701 \mathrm{~cm}^{-1} .{ }^{1} \mathrm{H}$ NMR $\left(400 \mathrm{MHz}, \mathrm{CDCl}_{3}, \mathrm{TMS}\right) \delta$ $7.74(\mathrm{~d}, J=8.0 \mathrm{~Hz}, 2 \mathrm{H}), 7.48(\mathrm{t}, J=8.0 \mathrm{~Hz}, 1 \mathrm{H}), 7.39(\mathrm{t}, J=8.0 \mathrm{~Hz}, 2 \mathrm{H}), 7.207 .13(\mathrm{~m}, 3 \mathrm{H}), 6.98-6.90$ $(\mathrm{m}, 2 \mathrm{H}), 3.21(\mathrm{~s}, 2 \mathrm{H}), 2.26-2.19(\mathrm{~m}, 2 \mathrm{H}), 1.92-1.84(\mathrm{~m}, 2 \mathrm{H}), 1.75-1.58(\mathrm{~m}, 4 \mathrm{H}) .{ }^{13} \mathrm{C}$ NMR $(100 \mathrm{MHz}$, $\left.\mathrm{CDCl}_{3}\right) \delta 206.1,138.3,137.4,131.5,129.7,128.9,128.1,128.1,126.4,60.5,45.1,36.2,24.9$. HRMS (APCI): calcd. for $\mathrm{C}_{19} \mathrm{H}_{21} \mathrm{O}\left([\mathrm{M}+\mathrm{H}]^{+}\right)$: 265.1592, found: 265.1581 .

\section{(1-Benzylcyclohexyl)(phenyl)methanone (3I)}

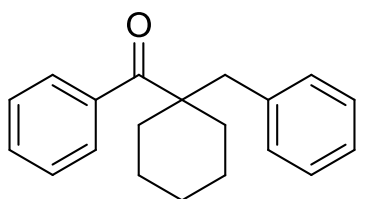

With general procedure $\mathbf{F}$, reaction of $\mathbf{1 l}(0.1 \mathrm{mmol})$ and $\mathbf{2 c}(0.3 \mathrm{mmol})$ provided the product $3 \mathrm{l}$ after flash column chromatography $(0.5 \mathrm{vol} \% \mathrm{EtOAc}$ in petroleum ether) as a colorless oil $(20.0 \mathrm{mg}, 72 \%) . \mathrm{R}_{f}=0.71(1.0 \mathrm{vol} \%$ EtOAc in petroleum ether). IR (neat), 2934, 2871, 1668, 1600, 1453, 1275, $1055,1032,751,701 \mathrm{~cm}^{-1} .{ }^{1} \mathrm{H}$ NMR $\left(400 \mathrm{MHz}, \mathrm{CDCl}_{3}, \mathrm{TMS}\right) \delta$ 7.35-7.32 (m, $1 \mathrm{H})$, 7.30-7.22 (m, 4H), 7.20-7.13 (m, 3H), 7.07-7.00 (m, 2H), $3.03(\mathrm{~s}, 2 \mathrm{H}), 2.18-2.15(\mathrm{~m}, 2 \mathrm{H})$, 1.49-1.43 (m, 2H), 1.41-1.35 (m, 3H), 1.21-1.07 (m, 3H). ${ }^{13} \mathrm{C}$ NMR (100 MHz, CDCl 3 ) $\delta 209.7,140.4$, 137.2, 130.5, 130.4, 128.1, 127.9, 127.3, 126.5, 53.6, 46.1, 34.9, 25.7, 23.0. HRMS (APCI): calcd. for $\mathrm{C}_{20} \mathrm{H}_{23} \mathrm{O}\left([\mathrm{M}+\mathrm{H}]^{+}\right): 279.1749$, found: 279.1749 .

\section{2,2-Dimethyl-3-phenyl-1-(4-vinylphenyl)propan-1-one (3m)}<smiles>C=Cc1ccc(C(=O)C(C)(C)Cc2ccccc2)cc1</smiles>

With general procedure $\mathbf{F}$, reaction of $\mathbf{1 m}(0.1 \mathrm{mmol})$ and $\mathbf{2 c}(0.3 \mathrm{mmol})$ provided the product $\mathbf{3 m}$ after flash column chromatography $(0.5 \mathrm{vol} \%$ EtOAc in petroleum ether) as a colorless oil $(18.5 \mathrm{mg}, 76 \%) . \mathrm{R}_{f}=0.76$ (1.0 vol \% EtOAc in petroleum ether). IR (neat), 2972, 2865, 2843, 1714, $1682,1646,1454,1345,1054,1032,1012,702 \mathrm{~cm}^{-1} .{ }^{1} \mathrm{H}$ NMR $(400 \mathrm{MHz}$, $\left.\mathrm{CDCl}_{3}, \mathrm{TMS}\right) \delta 7.54(\mathrm{~d}, J=8.3 \mathrm{~Hz}, 2 \mathrm{H}), 7.40(\mathrm{~d}, J=8.3 \mathrm{~Hz}, 2 \mathrm{H}), 7.24-7.20(\mathrm{~m}, 3 \mathrm{H}), 7.11-7.07(\mathrm{~m}$, $2 \mathrm{H}), 6.72(\mathrm{dd}, J=17.6,10.9 \mathrm{~Hz}, 1 \mathrm{H}), 5.82(\mathrm{~d}, J=17.6 \mathrm{~Hz}, 1 \mathrm{H}), 5.34(\mathrm{~d}, J=10.9 \mathrm{~Hz}, 1 \mathrm{H}), 3.08(\mathrm{~s}, 2 \mathrm{H})$, $1.31(\mathrm{~s}, 6 \mathrm{H}) .{ }^{13} \mathrm{C}$ NMR $\left(100 \mathrm{MHz}, \mathrm{CDCl}_{3}\right) \delta 208.5,139.9,138.3,137.9,136.0,130.5,128.2,128.0$, 126.4, 125.8, 115.9, 48.8, 46.4, 26.1. HRMS (APCI): calcd. for $\mathrm{C}_{19} \mathrm{H}_{21} \mathrm{O}\left([\mathrm{M}+\mathrm{H}]^{+}\right)$: 265.1592, found: 265.1590 .

\section{4,4-Dimethyl-1,5-diphenylpent-1-yn-3-one (3n)}

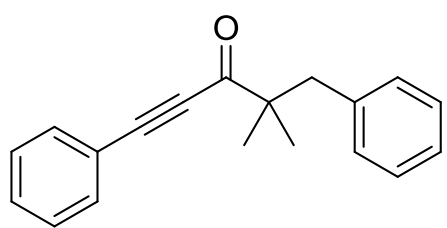

With general procedure $\mathbf{F}$, reaction of $\mathbf{1 n}{ }^{[16]}(0.1 \mathrm{mmol})$ and $\mathbf{2 c}(0.3 \mathrm{mmol})$ provided the product $3 \mathbf{n}$ after flash column chromatography $(0.5 \mathrm{vol} \%$ EtOAc in petroleum ether) as a colorless oil $(21.5 \mathrm{mg}, 82 \%) . \mathrm{R}_{f}=0.70$ (1.0 vol \% EtOAc in petroleum ether). IR (neat), 2980, 2196, 1661, 1454, $1275,1260,1012,749 \mathrm{~cm}^{-1} .{ }^{1} \mathrm{H}$ NMR $\left(400 \mathrm{MHz}, \mathrm{CDCl}_{3}, \mathrm{TMS}\right) \delta$ 7.62-7.57 (m, 2H), 7.49-7.44 (m, 1H), 7.42-7.37 (m, 2H), 7.29-7.25 (m, $2 \mathrm{H}), 7.24-7.19(\mathrm{~m}, 1 \mathrm{H}), 7.18-7.17(\mathrm{~m}, 2 \mathrm{H}), 3.00(\mathrm{~s}, 2 \mathrm{H}), 1.24(\mathrm{~s}, 6 \mathrm{H}) .{ }^{13} \mathrm{C}$ NMR $\left(100 \mathrm{MHz}, \mathrm{CDCl}_{3}\right) \delta$ 
193.5, 137.3, 133.0, 130.6, 130.3, 128.6, 128.1, 126.5, 120.2, 92.8, 86.4, 49.3, 45.2, 23.9. HRMS (APCI): calcd. for $\mathrm{C}_{19} \mathrm{H}_{19} \mathrm{O}\left([\mathrm{M}+\mathrm{H}]^{+}\right)$: 263.1436, found: 263.1436 .

\section{2,2-Dimethyl-3-phenyl-1-(thiophen-2-yl)propan-1-one (3o)}

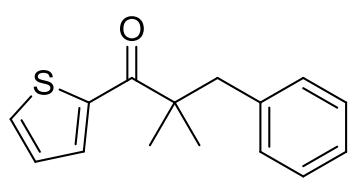

With general procedure $\mathbf{F}$, reaction of $\mathbf{1 0}(0.1 \mathrm{mmol})$ and $\mathbf{2 c}(0.3 \mathrm{mmol})$ provided the product 30 after flash column chromatography $(0.5$ vol \% EtOAc in petroleum ether) as a colorless oil $(20.7 \mathrm{mg}, 85 \%) . \mathrm{R}_{f}=0.81(1.0 \mathrm{vol} \%$ EtOAc in petroleum ether). IR (neat), 2981, 2783, 1646, 1516, 1454, 1411, $1275,1260,1056,1032,1012,749,702 \mathrm{~cm}^{-1} .{ }^{1} \mathrm{H}$ NMR $\left(400 \mathrm{MHz}, \mathrm{CDCl}_{3}\right.$, TMS) $\delta 7.75(\mathrm{~d}, \mathrm{~J}=4.0 \mathrm{~Hz}, 1 \mathrm{H}), 7.52(\mathrm{~d}, \mathrm{~J}=4.0 \mathrm{~Hz}, 1 \mathrm{H}), 7.17-7.10(\mathrm{~m}, 3 \mathrm{H}), 7.07-7.04(\mathrm{~m}, 1 \mathrm{H})$, 7.00-6.95 (m, 2H), $3.07(\mathrm{~s}, 2 \mathrm{H}), 1.29(\mathrm{~s}, 6 \mathrm{H}) . .{ }^{13} \mathrm{C} \mathrm{NMR}\left(100 \mathrm{MHz}, \mathrm{CDCl}_{3}\right) \delta 198.1,143.0,137.6$, $132.5, \quad 132.0,130.2,128.0,127.7,126.4,48.5,46.4,25.9$. HRMS (APCI): calcd. for $\mathrm{C}_{15} \mathrm{H}_{17} \mathrm{OS}\left([\mathrm{M}+\mathrm{H}]^{+}\right): 245.1000$, found: 245.1000 .

\section{2,2-Dimethyl-3-phenyl-1-(thiophen-3-yl)propan-1-one (3p)}

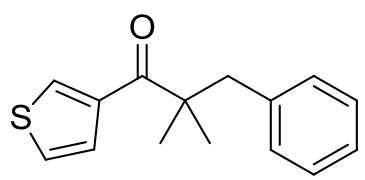

With general procedure $\mathbf{F}$, reaction of $\mathbf{1 p}(0.1 \mathrm{mmol})$ and $\mathbf{2 c}(0.3 \mathrm{mmol})$ provided the product $3 \mathbf{p}$ after flash column chromatography (0.5 vol \% EtOAc in petroleum ether) as a colorless oil $(21.1 \mathrm{mg}, 87 \%) . \mathrm{R}_{f}=0.79(1.0 \mathrm{vol} \%$ EtOAc in petroleum ether). IR (neat), 2981, 2783, 1661, 1507, 1454, 1275, 1056, 1032, 1015, 863, 750, $701 \mathrm{~cm}^{-1} .{ }^{1} \mathrm{H}$ NMR $\left(400 \mathrm{MHz}, \mathrm{CDCl}_{3}, \mathrm{TMS}\right) \delta$ $7.89(\mathrm{~d}, J=2.9 \mathrm{~Hz}, 1 \mathrm{H}), 7.47(\mathrm{~d}, J=5.1 \mathrm{~Hz}, 1 \mathrm{H}), 7.22(\mathrm{dd}, J=5.1,2.9 \mathrm{~Hz}, 1 \mathrm{H}), 7.17-7.10(\mathrm{~m}, 3 \mathrm{H})$, 7.00-6.94 (m, 2H), $3.02(\mathrm{~s}, 2 \mathrm{H}), 1.25(\mathrm{~s}, 6 \mathrm{H}) .{ }^{13} \mathrm{C}$ NMR $\left(100 \mathrm{MHz}, \mathrm{CDCl}_{3}\right) \delta 200.7,140.5,137.7$, $131.1, \quad 130.3,128.6,128.0,126.4,125.2,48.5,46.3,25.7$. HRMS (APCI): calcd. for $\mathrm{C}_{15} \mathrm{H}_{17} \mathrm{OS}\left([\mathrm{M}+\mathrm{H}]^{+}\right): 245.1000$, found: 245.0996 .

\section{Phenyl 2,2-dimethyl-3-phenylpropanoate (3q)}

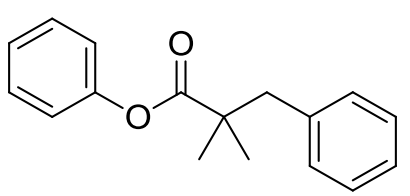

With general procedure $\mathbf{F}$, reaction of $\mathbf{1 q}(0.1 \mathrm{mmol})$ and $\mathbf{2 c}(0.3 \mathrm{mmol})$ provided the product $\mathbf{3 q}$ after flash column chromatography $(0.5$ vol $\%$ EtOAc in petroleum ether) as a colorless oil (19.8 mg, 78\%). $\mathrm{R}_{f}=0.65(1.0$ vol \% EtOAc in petroleum ether). IR (neat), 2980, 2924, 2840, 1742, 1275, $1259,1554,1032,1013,769,749,700 \mathrm{~cm}^{-1} .{ }^{1} \mathrm{H}$ NMR $\left(400 \mathrm{MHz}, \mathrm{CDCl}_{3}\right.$, TMS) $\delta$ 7.39-7.35 (m, 2H), 7.33-7.26 (m, 3H), 7.25-7.20 (m, 3H), 7.04-6.98 (m, 2H), 3.01 (s, 2H), $1.35(\mathrm{~s}, 6 \mathrm{H}) .{ }^{13} \mathrm{C}$ NMR $\left(100 \mathrm{MHz}, \mathrm{CDCl}_{3}\right) \delta 175.9,151.0,137.6,130.3,129.3,128.1,126.6,125.6$, 121.5, 46.3, 43.9, 25.1. HRMS (APCI): calcd. for $\mathrm{C}_{17} \mathrm{H}_{19} \mathrm{O}_{2}\left([\mathrm{M}+\mathrm{H}]^{+}\right)$: 255.1385, found: 255.1385 .

\section{4-Acetylphenyl 2,2-dimethyl-3-phenylpropanoate (3r)}

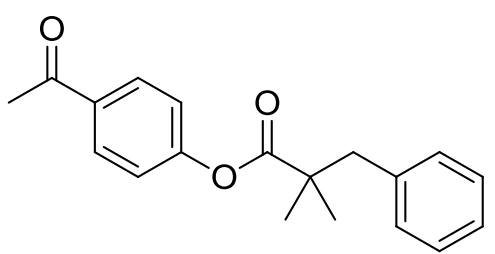

With general procedure $\mathbf{F}$, reaction of $\mathbf{1 r}(0.1 \mathrm{mmol})$ and $\mathbf{2 c}(0.3 \mathrm{mmol})$ provided the product $3 \mathbf{r}$ after flash column chromatography $(0.5 \mathrm{vol} \%$ EtOAc in petroleum ether) as a colorless oil $(20.1 \mathrm{mg}, 70 \%) . \mathrm{R}_{f}=0.60$ (1.0 vol \% EtOAc in petroleum ether). IR (neat), 2981, 2918, 2848, $1744,1682,1275,1260,1204,1159,1055,1032,1013,764,749,700$ $\mathrm{cm}^{-1} .{ }^{1} \mathrm{H}$ NMR $\left(400 \mathrm{MHz}, \mathrm{CDCl}_{3}\right.$, TMS $) \delta 7.98(\mathrm{~d}, J=8.8 \mathrm{~Hz}, 2 \mathrm{H})$, 7.35-7.26 (m, 3H), 7.22-7.19 (m, 2H), $7.09(\mathrm{~d}, J=8.8 \mathrm{~Hz}, 2 \mathrm{H}), 3.01(\mathrm{~s}, 2 \mathrm{H}), 2.60(\mathrm{~s}, 3 \mathrm{H}), 1.36(\mathrm{~s}, 6 \mathrm{H})$. ${ }^{13} \mathrm{C}$ NMR $\left(100 \mathrm{MHz}, \mathrm{CDCl}_{3}\right) \delta 196.9,175.4,154.7,137.3,134.6,130.2,129.9,128.2,126.2,121.7$, 46.3, 44.1, 26.6, 25.1. HRMS (APCI): calcd. for $\mathrm{C}_{19} \mathrm{H}_{21} \mathrm{O}_{3}\left([\mathrm{M}+\mathrm{H}]^{+}\right)$: 297.1491, found: 297.1490. 


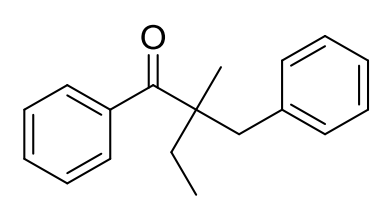

With general procedure $\mathbf{F}$, reaction of $1 \mathbf{s}(0.1 \mathrm{mmol})$ and $\mathbf{2 c}(0.3 \mathrm{mmol})$ provided the product $3 \mathrm{~s}$ after flash column chromatography (1.0 vol \% EtOAc in petroleum ether) as a colorless oil $(20.1 \mathrm{mg}, 80 \%) . \mathrm{R}_{f}=0.60(1.0 \mathrm{vol} \%$ EtOAc in petroleum ether). IR (neat), 2967, 2933, 2875, 1682, 1596, 1459, $1217,1181,734,701 \mathrm{~cm}^{-1} .{ }^{1} \mathrm{H}$ NMR $\left(400 \mathrm{MHz}, \mathrm{CDCl}_{3}\right) \delta$ 7.48-7.40 (m, 3H), 7.37-7.35 (m, 2H), 7.26-7.17 (m, 3H), 7.15-7.07 (m, 2H), $3.25(\mathrm{~d}, J=13.4 \mathrm{~Hz}, 1 \mathrm{H}), 2.87(\mathrm{~d}, J=13.4$ $\mathrm{Hz}, 1 \mathrm{H}), 2.05(\mathrm{~m}, 1 \mathrm{H}), 1.69-1.61(\mathrm{~m}, 1 \mathrm{H}), 1.20(\mathrm{~s}, 3 \mathrm{H}), 0.89(\mathrm{t}, J=7.5 \mathrm{~Hz}, 3 \mathrm{H}) .{ }^{13} \mathrm{C} \mathrm{NMR}(101 \mathrm{MHz}$, $\left.\mathrm{CDCl}_{3}\right) \delta 209.4,140.2,137.9,130.6,130.5,128.0,128.0,127.2,126.4,52.9,45.0,32.6,22.3,9.1$. HRMS (EI): calcd. for $\mathrm{C}_{18} \mathrm{H}_{20} \mathrm{O}\left([\mathrm{M}]^{+}\right)$: 252.1514, found: 252.1518 .

\section{2-Benzyl-2-ethyl-1-phenylbutan-1-one (3t)}

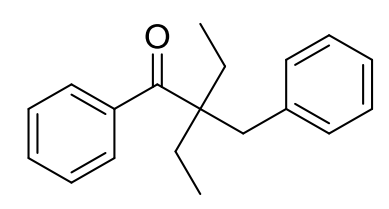

With general procedure $\mathbf{F}$, reaction of $\mathbf{1 t}(0.1 \mathrm{mmol})$ and $2 \mathbf{c}(0.3 \mathrm{mmol})$ provided the product $3 \mathbf{t}$ after flash column chromatography (1.0 vol \% EtOAc in petroleum ether) as a colorless oil $(21.3 \mathrm{mg}, 80 \%) . \mathrm{R}_{f}=0.60(1.0 \mathrm{vol} \%$ EtOAc in petroleum ether). IR (neat), 2974, 2939, 2880, 1678, 1569, 1455, 1229, 1097, 854, $721 \mathrm{~cm}^{-1} .{ }^{1} \mathrm{H}$ NMR $\left(400 \mathrm{MHz}, \mathrm{CDCl}_{3}, \mathrm{TMS}\right) \delta 7.42(\mathrm{~d}, J=$ $6.8 \mathrm{~Hz}, 3 \mathrm{H}), 7.36-7.32(\mathrm{~m}, 2 \mathrm{H}), 7.27-7.19(\mathrm{~m}, 3 \mathrm{H}), 7.12(\mathrm{~d}, J=6.8 \mathrm{~Hz}, 2 \mathrm{H}), 3.07(\mathrm{~s}, 2 \mathrm{H}), 1.75(\mathrm{q}, J=$ $7.2 \mathrm{~Hz}, 4 \mathrm{H}), 0.89(\mathrm{t}, J=7.2 \mathrm{~Hz}, 6 \mathrm{H}) .{ }^{13} \mathrm{C}$ NMR $\left(100 \mathrm{MHz}, \mathrm{CDCl}_{3}\right) \delta 209.7,140.6,138.1,130.5,130.4$, 128.1, 128.0, 127.0, 126.3, 56.6, 39.1, 27.2, 8.7. HRMS (EI): calcd. for $\mathrm{C}_{19} \mathrm{H}_{22} \mathrm{O}\left([\mathrm{M}]^{+}\right): 266.1671$, found: 266.1662 .

\section{2-benzyl-2-methylcyclohexan-1-one (3u)}<smiles>CC1(Cc2ccccc2)CCCCC1=O</smiles>

With general procedure $\mathbf{F}$, reaction of $\mathbf{1} \mathbf{u}^{[17]}(0.1 \mathrm{mmol})$ and $\mathbf{2 c}(0.3 \mathrm{mmol})$ provided the product 3u after flash column chromatography (4.0 vol \% EtOAc in petroleum ether) as a colorless oil $(15.8 \mathrm{mg}, 78 \%)$. $\mathrm{R}_{f}=0.61$ (4.0 vol \% EtOAc in petroleum ether). IR (neat), 3306, 2934, 2864, 1712, 1682, 1539, 1377, 1236, 1118, $903 \mathrm{~cm}^{-1}$. ${ }^{1} \mathrm{H}$ NMR $\left(400 \mathrm{MHz}, \mathrm{CDCl}_{3}\right.$, TMS) $\delta$ 7.28-7.19 (m, 3H), $7.11(\mathrm{~d}, J=7.2 \mathrm{~Hz}, 2 \mathrm{H}), 2.88(\mathrm{~s}, 2 \mathrm{H})$, 2.55-2.42 (m, 2H), 1.85-1.54 (m, 6H), 1.03 (s, 3H). $\left.{ }^{13} \mathrm{C} \mathrm{NMR} \mathrm{(100} \mathrm{MHz,} \mathrm{CDCl}_{3}\right) \delta 215.5,130.5,127.9$, 126.3, 43.1, 38.9, 38.1, 27.3, 22.8, 21.1. HRMS (EI): calcd. for $\mathrm{C}_{14} \mathrm{H}_{18} \mathrm{O}\left([\mathrm{M}]^{+}\right)$: 202.1358, found: 202.1362 .

\section{2-benzyl-2-methylcyclohexan-1-one (3v)}

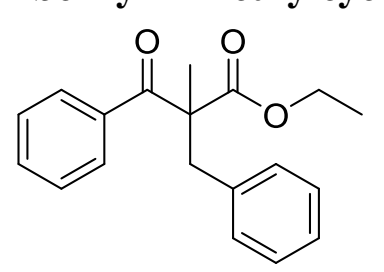

With general procedure $\mathbf{F}$, reaction of $\mathbf{1} \mathbf{v}^{[18]}(0.1 \mathrm{mmol})$ and $\mathbf{2 c}(0.3 \mathrm{mmol})$ provided the product $3 \mathrm{v}$ after flash column chromatography (4.0 vol \% EtOAc in petroleum ether) as white solid $(25.2 \mathrm{mg}, 85 \%)$. $\mathrm{R}_{f}=0.60(4.0 \mathrm{vol} \%$ EtOAc in petroleum ether). IR (neat), 3062, 2936, 1754, 1723, 1693, 1597, 1464, $1375,1258,1108 \mathrm{~cm}^{-1} .{ }^{1} \mathrm{H}$ NMR $\left(400 \mathrm{MHz}, \mathrm{CDCl}_{3}\right) \delta 7.86(\mathrm{~d}, J=7.7 \mathrm{~Hz}, 2 \mathrm{H})$, 7.55-7.52 (m, 1H), 7.45-7.41 (m, 2H), 7.22 (m, 3H), $7.02(\mathrm{~d}, J=6.4 \mathrm{~Hz}, 2 \mathrm{H})$, $4.09(\mathrm{q}, J=7.0 \mathrm{~Hz}, 2 \mathrm{H}), 3.42(\mathrm{~d}, J=13.8 \mathrm{~Hz}, 1 \mathrm{H}), 3.33(\mathrm{~d}, J=13.8 \mathrm{~Hz}, 1 \mathrm{H}), 1.48(\mathrm{~s}, 3 \mathrm{H}), 1.02(\mathrm{t}, J=$ $7.0 \mathrm{~Hz}, 3 \mathrm{H}) .{ }^{13} \mathrm{C}$ NMR $\left(100 \mathrm{MHz}, \mathrm{CDCl}_{3}\right) \delta 197.3,173.6,136.2,135.8,132.7,130.3,128.6,128.5$, 128.1, 126.8, 61.4, 58.2, 42.2, 21.1, 13.7. HRMS (EI): calcd. for $\mathrm{C}_{19} \mathrm{H}_{20} \mathrm{O}_{3}\left([\mathrm{M}]^{+}\right)$: 296.1412, found: 296.1419.

\section{2-Methyl-2-nitropropyl)benzene (3w)}

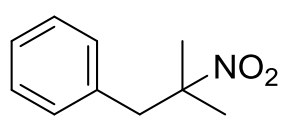

With general procedure $\mathbf{F}$, reaction of 2-bromo-2-nitropropane $(0.1 \mathrm{mmol})$ and $\mathbf{2 c}$ $(0.3 \mathrm{mmol})$ provided the product $\mathbf{3} \mathbf{w}$ after flash column chromatography $(0.5 \mathrm{vol} \%$ EtOAc in petroleum ether) as a colorless oil $(9.2 \mathrm{mg}, 51 \%) . \mathrm{R}_{f}=0.68(1.0 \mathrm{vol} \%$ 
EtOAc in petroleum ether). ${ }^{1} \mathrm{H}$ NMR (400 MHz, $\left.\mathrm{CDCl}_{3}, \mathrm{TMS}\right) \delta$ 7.34-7.28 (m, 3H), 7.11-7.10 (m, 2H), 3.20 (s, 2H), $1.58(\mathrm{~s}, 6 \mathrm{H}) .{ }^{13} \mathrm{C}$ NMR (100 MHz, $\left.\mathrm{CDCl}_{3}\right) \delta$ 135.0, 130.1, 128.5, 127.5, 88.6, 46.8, 25.6.

\section{2-Benzyl-3-methoxy-2-methyl-1-phenylpropan-1-one (3x)}

With general procedure $\mathbf{F}$, reaction of $\mathbf{1 x}(0.1 \mathrm{mmol})$ and $\mathbf{2 c}(0.3 \mathrm{mmol})$ provided<smiles>COCC(C)(Cc1ccccc1)C(=O)c1ccccc1</smiles>
the product $3 \mathbf{x}$ after flash column chromatography $(0.5$ vol \% EtOAc in petroleum ether) as a colorless oil $(19.6 \mathrm{mg}, 73 \%) . \mathrm{R}_{f}=06$ (1.0 vol \% EtOAc in petroleum ether). IR (neat), 2986, 2932, 2898, 2828, 1682, 1597, 1446, 1381, 1291, 1226 $\mathrm{cm}^{-1} .{ }^{1} \mathrm{H}$ NMR $\left(400 \mathrm{MHz}, \mathrm{CDCl}_{3}\right.$, TMS) $\delta$ 7.50-7.39 (m, 3H), 7.39-7.29 (m, 2H), 7.26-7.18 (m, 3H), $7.12(\mathrm{~d}, J=6.8 \mathrm{~Hz}, 2 \mathrm{H}), 3.46(\mathrm{~d}, J=9.0 \mathrm{~Hz}, 1 \mathrm{H}), 3.39$ (d, J = 9.0 Hz, 1H), 3.30 (s, $3 \mathrm{H}), 3.11-3.01(\mathrm{~m}, 2 \mathrm{H}), 1.24(\mathrm{~s}, 3 \mathrm{H}) .{ }^{13} \mathrm{C} \mathrm{NMR}\left(100 \mathrm{MHz}, \mathrm{CDCl}_{3}\right) \delta 209.3,140.3,137.3,130.4,130.2,128.1$, 128.0, 126.8, 126.0, 58.8, 53.1, 41.0, 29.7, 20.6. HRMS (EI): calcd. for $\mathrm{C}_{18} \mathrm{H}_{20} \mathrm{O}_{2}\left([\mathrm{M}]^{+}\right): 268.1463$, found: 268.1468 .

\section{3-(4-Methoxyphenyl)-2,2-dimethyl-1-phenylpropan-1-one (3y)}

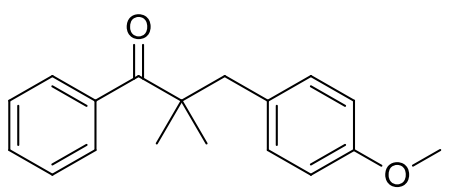

With general procedure $\mathbf{F}$, reaction of $\mathbf{1 a}(0.1 \mathrm{mmol})$ and $\mathbf{2 e}(0.3 \mathrm{mmol})$ provided the product $3 \mathbf{y}$ after flash column chromatography $(0.5 \mathrm{vol} \%$ EtOAc in petroleum ether) as a colorless oil $(19.0 \mathrm{mg}, 71 \%) . \mathrm{R}_{f}=0.60$ (1.0 vol \% EtOAc in petroleum ether). IR (neat), 2981, 22843, 1736, 1673, $1512,1454,1275,1260,1055,1032,1012,749 \mathrm{~cm}^{-1} .{ }^{1} \mathrm{H}$ NMR $(400 \mathrm{MHz}$, $\left.\mathrm{CDCl}_{3}, \mathrm{TMS}\right) \delta 7.52(\mathrm{~d}, J=7.5 \mathrm{~Hz}, 2 \mathrm{H}), 7.40(\mathrm{dt}, J=14.5,7.5 \mathrm{~Hz}, 3 \mathrm{H}), 7.01(\mathrm{~d}, J=8.3 \mathrm{~Hz}, 2 \mathrm{H}), 6.78$ $(\mathrm{d}, J=8.3 \mathrm{~Hz}, 2 \mathrm{H}), 3.78(\mathrm{~s}, 3 \mathrm{H}), 3.01(\mathrm{~s}, 2 \mathrm{H}), 1.28(\mathrm{~s}, 6 \mathrm{H}) .{ }^{13} \mathrm{C} \mathrm{NMR}\left(100 \mathrm{MHz}, \mathrm{CDCl}_{3}\right) \delta 209.7$, 158.2, 139.5, 131.4, 130.6, 129.9, 128.0, 127.5, 113.4, 55.2, 48.9, 45.4, 26.0. HRMS (APCI): calcd. for $\mathrm{C}_{18} \mathrm{H}_{21} \mathrm{O}_{2}\left([\mathrm{M}+\mathrm{H}]^{+}\right): 269.1542$, found: 269.1540 .

\section{1-(4-Fluorophenyl)-3-(4-methoxyphenyl)-2,2-dimethylpropan-1-one (3z)}

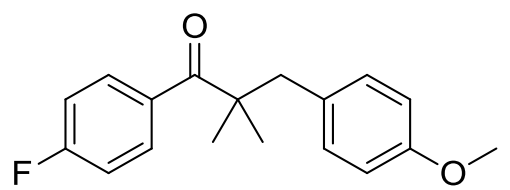

With general procedure $\mathbf{F}$, reaction of $\mathbf{1 d}(0.1 \mathrm{mmol})$ and $\mathbf{2 e}(0.3 \mathrm{mmol})$ provided the product $3 \mathbf{z}$ after flash column chromatography $(0.5 \mathrm{vol} \%$ EtOAc in petroleum ether) as a colorless oil (20.1 mg, 72\%). $\mathrm{R}_{f}=0.70$ (1.0 vol \% EtOAc in petroleum ether). IR (neat), 2980, 2916, 1714, 1646, 1506, 1454, 1275, 1260, 1054, 1032, 1012, 767, 749, $700 \mathrm{~cm}^{-1}$. ${ }^{1} \mathrm{H}$ NMR $\left(400 \mathrm{MHz}, \mathrm{CDCl}_{3}, \mathrm{TMS}\right) \delta$ 7.61-7.58 (m, 2H), 7.08-6.98 (m, 4H), 6.80-6.75 (m, 2H), 3.77 (s, $3 \mathrm{H}), 3.01(\mathrm{~s}, 2 \mathrm{H}), 1.29(\mathrm{~s}, 6 \mathrm{H}) .{ }^{13} \mathrm{C} \mathrm{NMR}\left(100 \mathrm{MHz}, \mathrm{CDCl}_{3}\right) \delta 207.6,164.1(\mathrm{~d}, J=250 \mathrm{~Hz}), 158.3$, $135.3(\mathrm{~d}, J=2 \mathrm{~Hz}), 131.3,130.3(\mathrm{~d}, J=8 \mathrm{~Hz}), 129.8,115.1(\mathrm{~d}, J=21 \mathrm{~Hz}), 113.5,55.2,48.9,45.6$, 26.2. HRMS (APCI): calcd. for $\mathrm{C}_{18} \mathrm{H}_{20} \mathrm{FO}_{2}\left([\mathrm{M}+\mathrm{H}]^{+}\right)$: 287.1447, found: 287.1446.

\section{1-Mesityl-3-(4-methoxyphenyl)-2,2-dimethylpropan-1-one (3aa)}

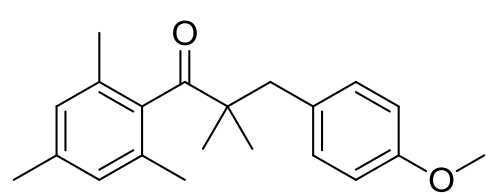

With general procedure $\mathbf{F}$, reaction of $\mathbf{1 g}(0.1 \mathrm{mmol})$ and $\mathbf{2 e}(0.3 \mathrm{mmol})$ provided the product 3aa after flash column chromatography $(0.5 \mathrm{vol} \%$ EtOAc in petroleum ether) as a colorless oil (21.4 mg, 69\%). $\mathrm{R}_{f}=0.70$ (1.0 vol \% EtOAc in petroleum ether). IR (neat), 3006, 2981, 2865, 2843, 1683, 1610, 1511, 1454, 1345, 1275, 1171, 1054, 1032, 1012, $749,702 \mathrm{~cm}^{-1} .{ }^{1} \mathrm{H}$ NMR $\left(400 \mathrm{MHz}, \mathrm{CDCl}_{3}\right) \delta 7.09(\mathrm{~d}, J=8.6 \mathrm{~Hz}, 2 \mathrm{H}), 6.83-6.81(\mathrm{~m}, 4 \mathrm{H}), 3.79(\mathrm{~s}, 3 \mathrm{H})$, $2.90(\mathrm{~s}, 2 \mathrm{H}), 2.27(\mathrm{~s}, 3 \mathrm{H}), 2.16(\mathrm{~s}, 6 \mathrm{H}), 1.11(\mathrm{~s}, 6 \mathrm{H}) .{ }^{13} \mathrm{C} \mathrm{NMR}\left(100 \mathrm{MHz}, \mathrm{CDCl}_{3}\right) \delta 218.8,158.2$, $139.4,137.5,132.3,132.0,129.5,128.5,113.3,55.2,49.1,44.1,24.7,20.9$, 20.4. HRMS (APCI): calcd. for $\mathrm{C}_{21} \mathrm{H}_{26} \mathrm{O}_{2}\left([\mathrm{M}+\mathrm{H}]^{+}\right)$: 311.2011, found: 311.2013. 


\section{3-(4-Methoxyphenyl)-2,2-dimethyl-1-(thiophen-2-yl)propan-1-one (3ab)}

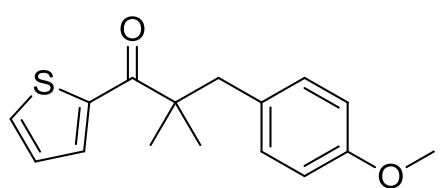

With general procedure $\mathbf{F}$, reaction of $\mathbf{1 0}(0.1 \mathrm{mmol})$ and $\mathbf{2 e}(0.3 \mathrm{mmol})$ provided the product $3 \mathbf{a b}$ after flash column chromatography $(0.5 \mathrm{vol} \%$ EtOAc in petroleum ether) as a colorless oil $(23.3 \mathrm{mg}: 85 \%) . \mathrm{R}_{f}=0.68$ (1.0 vol \% EtOAc in petroleum ether). IR (neat), 2981, 2928, 2869, 2848, $1642,1510,1454,1275,1260,1055,1032,1012,749,706 \mathrm{~cm}^{-1} .{ }^{1} \mathrm{H}$ NMR $\left(400 \mathrm{MHz}, \mathrm{CDCl}_{3}, \mathrm{TMS}\right) \delta 7.83(\mathrm{~d}, J=4.5 \mathrm{~Hz}, 1 \mathrm{H}), 7.60(\mathrm{~d}, J=4.5 \mathrm{~Hz}, 1 \mathrm{H}), 7.15-7.12(\mathrm{~m}, 1 \mathrm{H}), 6.95$ $(\mathrm{d}, J=8.6 \mathrm{~Hz}, 2 \mathrm{H}), 6.76(\mathrm{~d}, J=8.6 \mathrm{~Hz}, 2 \mathrm{H}), 3.76(\mathrm{~s}, 3 \mathrm{H}), 3.08(\mathrm{~s}, 2 \mathrm{H}), 1.35(\mathrm{~s}, 6 \mathrm{H}) .{ }^{13} \mathrm{C} \mathrm{NMR}(100$ $\left.\mathrm{MHz}, \mathrm{CDCl}_{3}\right) \delta 198.3,158.2,143.1,132.5,132.0,131.1,130.0,127.7,113.4,55.2,48.6,45.9,25.9$. HRMS (APCI): calcd. for $\mathrm{C}_{16} \mathrm{H}_{19} \mathrm{O}_{2} \mathrm{~S}\left([\mathrm{M}+\mathrm{H}]^{+}\right)$: 275.1106, found: 275.1100 .

\section{3-(4-Chlorophenyl)-2,2-dimethyl-1-phenylpropan-1-one (3ac)}

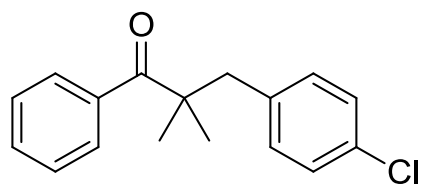

With general procedure $\mathbf{F}$, reaction of $\mathbf{1 a}(0.1 \mathrm{mmol})$ and $\mathbf{2 f}(0.3 \mathrm{mmol})$ provided the product 3ac after flash column chromatography $(0.5$ vol $\%$ EtOAc in petroleum ether) as a colorless oil $(22.0 \mathrm{mg}, 81 \%) . \mathrm{R}_{f}=0.67(1.0$ vol \% EtOAc in petroleum ether). IR (neat), 3006, 2981, 2840, 1459, 1275, 1260, 1054, 1032, 1014, 764, 749, $700 \mathrm{~cm}^{-1} .{ }^{1} \mathrm{H}$ NMR (400 MHz, $\mathrm{CDCl}_{3}$, TMS) $\delta$ 7.54-7.50 (m, 2H), 7.47-7.43 (m, 1H), 7.40-7.36 (m, 2H), 7.21 (d, $J=8.4 \mathrm{~Hz}, 2 \mathrm{H}), 7.03(\mathrm{~d}, J=$ $8.4 \mathrm{~Hz}, 2 \mathrm{H}), 3.04(\mathrm{~s}, 2 \mathrm{H}), 1.29(\mathrm{~s}, 6 \mathrm{H}) .{ }^{13} \mathrm{C} \mathrm{NMR}\left(100 \mathrm{MHz}, \mathrm{CDCl}_{3}\right) \delta 209.1,139.3,136.4,132.3$, $131.8,130.8,128.1,128.1,127.4,48.7,45.5,26.1$. HRMS (APCI): calcd. for $\mathrm{C}_{17} \mathrm{H}_{18} \mathrm{ClO}\left([\mathrm{M}+\mathrm{H}]^{+}\right)$: 273.1046, found: 273.1046 .

\section{3-(4-Chlorophenyl)-2,2-dimethyl-1-(thiophen-2-yl)propan-1-one (3ad)}

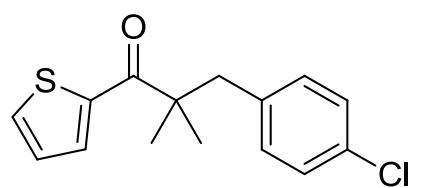

With general procedure $\mathbf{F}$, reaction of $\mathbf{1 0}(0.1 \mathrm{mmol})$ and $\mathbf{2 f}(0.3 \mathrm{mmol})$ provided the product 3ad after flash column chromatography $(0.5 \mathrm{vol} \%$ EtOAc in petroleum ether) as a colorless oil $(22.2 \mathrm{mg}, 80 \%) . \mathrm{R}_{f}=0.60(1.0$ vol \% EtOAc in petroleum ether). IR (neat), 2981, 2865, 2842, 1655, 1644, $1408,1275,1260,1032,1014,764,749 \mathrm{~cm}^{-1} .{ }^{1} \mathrm{H}$ NMR $\left(400 \mathrm{MHz}, \mathrm{CDCl}_{3}\right.$, TMS) $\delta 7.82(\mathrm{dd}, J=4.0,0.8 \mathrm{~Hz}, 1 \mathrm{H}), 7.61(\mathrm{dd}, J=5.0,0.8 \mathrm{~Hz}, 1 \mathrm{H}), 7.19(\mathrm{~d}, J=8.4 \mathrm{~Hz}, 2 \mathrm{H}), 7.14$ $(\mathrm{dd}, J=5.0,4.0 \mathrm{~Hz}, 1 \mathrm{H}), 6.98(\mathrm{~d}, J=8.4 \mathrm{~Hz}, 2 \mathrm{H}), 3.10(\mathrm{~s}, 2 \mathrm{H}), 1.36(\mathrm{~s}, 6 \mathrm{H}) .{ }^{13} \mathrm{C}$ NMR $(100 \mathrm{MHz}$, $\left.\mathrm{CDCl}_{3}\right) \delta 197.8,142.8,136.1,132.7,132.4,132.0,131.5,128.1,127.8,48.4,46.0,25.9$. HRMS (APCI): calcd. for $\mathrm{C}_{15} \mathrm{H}_{16} \mathrm{ClOS}\left([\mathrm{M}+\mathrm{H}]^{+}\right)$: 279.0610, found: 279.0611 .

\section{2,2-Dimethyl-1-phenyl-3-(thiophen-2-yl)propan-1-one (3ae)}

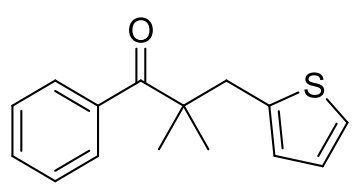

With general procedure $\mathbf{F}$, reaction of $\mathbf{1 a}(0.1 \mathrm{mmol})$ and $\mathbf{2 g}(0.3 \mathrm{mmol})$ provided the product 3ae after flash column chromatography $(0.5$ vol \% EtOAc in petroleum ether) as a colorless oil $(19.5 \mathrm{mg}, 80 \%) . \mathrm{R}_{f}=0.58(1.0 \mathrm{vol} \%$ EtOAc in petroleum ether). IR (neat), 2980, 2864, 2843, 1669, 1454, 1275, 1054, 1032, 1014, 765, 749, $691 \mathrm{~cm}^{-1} .{ }^{1} \mathrm{H}$ NMR (400 MHz, $\left.\mathrm{CDCl}_{3}, \mathrm{TMS}\right) \delta$ $7.50(\mathrm{~d}, J=7.5 \mathrm{~Hz}, 2 \mathrm{H}), 7.38(\mathrm{t}, J=7.5 \mathrm{~Hz}, 1 \mathrm{H}), 7.31(\mathrm{t}, J=7.5 \mathrm{~Hz}, 2 \mathrm{H}), 7.04(\mathrm{~d}, J=4.0 \mathrm{~Hz}, 1 \mathrm{H})$, 6.84-6.80 (m, 1H), $6.67(\mathrm{~d}, J=4.0 \mathrm{~Hz}, 1 \mathrm{H}), 3.20(\mathrm{~s}, 2 \mathrm{H}), 1.30(\mathrm{~s}, 6 \mathrm{H}) .{ }^{13} \mathrm{C} \mathrm{NMR}\left(100 \mathrm{MHz}, \mathrm{CDCl}_{3}\right) \delta$ 208.9, 140.0, 139.1, 130.8, 128.1, 127.6, 127.1, 126.5, 124.1, 48.8, 40.5, 26.1. HRMS (APCI): calcd. for $\mathrm{C}_{15} \mathrm{H}_{17} \mathrm{OS}\left([\mathrm{M}+\mathrm{H}]^{+}\right): 245.1000$, found: 245.1003 .

\section{2,2-Dimethyl-1,3-diphenylbutan-1-one (3af)}<smiles>CC(c1ccccc1)C(C)(C)C(=O)c1ccccc1</smiles>

With general procedure $\mathbf{F}$, reaction of $\mathbf{1 a}(0.1 \mathrm{mmol})$ and $\mathbf{2 h}(0.3 \mathrm{mmol})$ provided the product 3 af after flash column chromatography ( $0.5 \mathrm{vol} \%$ EtOAc in petroleum ether) as a colorless oil $(18.1 \mathrm{mg}, 72 \%) . \mathrm{R}_{f}=0.62(1.0 \mathrm{vol} \%$ 
EtOAc in petroleum ether). IR (neat), 2980, 2865, 2843, 1685, 1454, 1345, 1275, 1055, 1032, 1012, 765, 749, $702 \mathrm{~cm}^{-1} .{ }^{1} \mathrm{H}$ NMR (400 MHz, $\left.\mathrm{CDCl}_{3}, \mathrm{TMS}\right) \delta 7.41-7.38(\mathrm{~m}, 3 \mathrm{H}), 7.31-7.28(\mathrm{~m}, 2 \mathrm{H})$, 7.23-7.19 (m, 2H), 7.17-7.13 (m, 3H), $3.49(\mathrm{q}, J=7.2 \mathrm{~Hz}, 1 \mathrm{H}), 1.21(\mathrm{~d}, J=7.2 \mathrm{~Hz}, 3 \mathrm{H}), 1.15(\mathrm{~s}, 3 \mathrm{H})$, $1.09(\mathrm{~s}, 3 \mathrm{H}) .{ }^{13} \mathrm{C}$ NMR $\left(100 \mathrm{MHz}, \mathrm{CDCl}_{3}\right) \delta 210.1,142.5,139.9,130.5,129.5,128.0,127.8,127.2$, 126.6, 51.7, 45.6, 25.0, 21.7, 16.0. HRMS (APCI): calcd. for $\mathrm{C}_{18} \mathrm{H}_{21} \mathrm{O}\left([\mathrm{M}+\mathrm{H}]^{+}\right)$: 253.1592, found: 253.1593.

\section{1-(3-Chlorophenyl)-2,2-dimethyl-3-phenylbutan-1-one (3ag)}<smiles>CC(c1ccccc1)C(C)(C)C(=O)c1cccc(Cl)c1</smiles>

With general procedure $\mathbf{F}$, reaction of $\mathbf{1 f}(0.1 \mathrm{mmol})$ and $\mathbf{2 h}(0.3 \mathrm{mmol})$ provided the product 3ag after flash column chromatography $(0.5 \mathrm{vol} \%$ EtOAc in petroleum ether) as a colorless oil $(21.5 \mathrm{mg}, 75 \%) . \mathrm{R}_{f}=0.65(1.0$ vol \% EtOAc in petroleum ether). IR (neat), 2980, 2922, 2843, 1644, 1471, $1457,1275,1260,1054,1032,1014,896,756,750 \mathrm{~cm}^{-1} .{ }^{1} \mathrm{H}$ NMR $(400$ $\left.\mathrm{MHz}, \mathrm{CDCl}_{3}, \mathrm{TMS}\right) \delta 7.40(\mathrm{~d}, J=7.6 \mathrm{~Hz}, 1 \mathrm{H}), 7.33-7.26(\mathrm{~m}, 5 \mathrm{H}), 7.20(\mathrm{~d}, J=7.6 \mathrm{~Hz}, 2 \mathrm{H}), 3.49(\mathrm{q}, J$ $=7.2 \mathrm{~Hz}, 1 \mathrm{H}), 1.29(\mathrm{~d}, J=7.2 \mathrm{~Hz}, 3 \mathrm{H}), 1.22(\mathrm{~s}, 3 \mathrm{H}), 1.15(\mathrm{~s}, 3 \mathrm{H}) .{ }^{13} \mathrm{C} \mathrm{NMR}\left(100 \mathrm{MHz}, \mathrm{CDCl}_{3}\right) \delta$ 209.3, 142.2, 141.5, 134.2, 130.4, 129.4, 129.3, 127.9, 127.3, 126.7, 125.1, 51.9, 45.8, 24.7, 22.1, 15.9. HRMS (APCI): calcd. for $\mathrm{C}_{18} \mathrm{H}_{20} \mathrm{ClO}\left([\mathrm{M}+\mathrm{H}]^{+}\right)$: 287.1203, found: 287.1201.

\section{2,6-Dimethyl-4-(2-phenylpropan-2-yl)-1,4-dihydropyridine-3,5-dicarbonitrile(7a)}

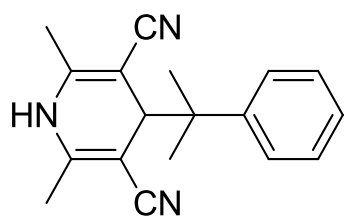

With general procedure $\mathbf{E}$, reaction of 2-methyl-2-phenylpropanal $(10 \mathrm{mmol})$ and $\beta$-aminocrotonitrile $(20 \mathrm{mmol})$ provided $\mathbf{7 a}$ after flash column chromatography (60\% EtOAc in petroleum ether) as a white solid (1.5 g, $55 \%)$. $\mathrm{R}_{f}=0.40$ (50 vol \% EtOAc/petroleum ether). M.P. $150-152{ }^{\circ} \mathrm{C}$. IR (neat) 3343, 2987, 2957, 2199, 1754, 1741, 1514, 1436, 1432, $1259 \mathrm{~cm}^{-1},{ }^{1} \mathrm{H}$ NMR (400 $\left.\mathrm{MHz}, \mathrm{CDCl}_{3}, \mathrm{TMS}\right) \delta$ 7.26-7.17 (m, 5H), $6.23(\mathrm{~s}, 1 \mathrm{H}), 3.22(\mathrm{~s}, 1 \mathrm{H}), 1.95(\mathrm{~s}, 6 \mathrm{H})$, $1.35(\mathrm{~s}, 6 \mathrm{H}) .{ }^{13} \mathrm{C} \mathrm{NMR}\left(100 \mathrm{MHz}, \mathrm{CDCl}_{3}\right) \delta 148.6,144.2,127.8,127.0,126.7,119.7,81.0,47.6,46.6$, 24.6, 18.3. HRMS (ESI): calcd. for $\mathrm{C}_{18} \mathrm{H}_{19} \mathrm{~N}_{3} \mathrm{Na}\left([\mathrm{M}+\mathrm{Na}]^{+}\right)$: 300.1477 , found: 300.1466 .

\section{4-(2-(4-Methoxyphenyl)propan-2-yl)-2,6-dimethyl-1,4-dihydropyridine-3,5-dicarbonitrile(7b)}

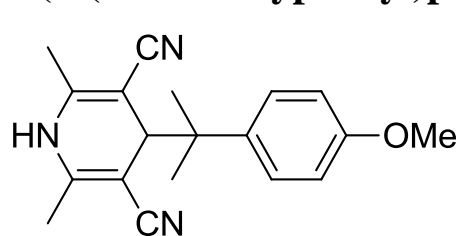

With general procedure $\mathbf{E}$, reaction of 2-(4-methoxyphenyl)-2-methylpropanal $\quad\left(\begin{array}{ll}10 & \mathrm{mmol})\end{array}\right.$ and $\beta$-aminocrotonitrile $(20 \mathrm{mmol})$ provided $\mathbf{7 b}$ after flash column chromatography (60\% EtOAc in petroleum ether) as a white solid (2.0 g, $62 \%) . \mathrm{R}_{f}=0.40$ (50 vol \% EtOAc/petroleum ether). M.P. $169-171{ }^{\circ} \mathrm{C} . \mathrm{IR}$ (neat) $3671,3648,3295,2989,2800,2200,1751,1712,1681,1514,1463,1259,1257, \mathrm{~cm}^{-1},{ }^{1} \mathrm{H}$ NMR (400 MHz, CDCl 3 , TMS) $\delta 7.20(\mathrm{~d}, J=8.8 \mathrm{~Hz}, 2 \mathrm{H}), 6.81(\mathrm{~d}, J=8.8 \mathrm{~Hz}, 2 \mathrm{H}), 3.78(\mathrm{~s}, 3 \mathrm{H}), 3.23$ $(\mathrm{s}, 1 \mathrm{H}), 2.01(\mathrm{~s}, 6 \mathrm{H}), 1.38(\mathrm{~s}, 6 \mathrm{H}) .{ }^{13} \mathrm{C}$ NMR $\left(100 \mathrm{MHz}, \mathrm{CDCl}_{3}\right) \delta 158.5,148.7,136.3,128.0,119.8$, 113.1, 80.9, 55.2, 47.6, 46.0, 24.8, 18.2. HRMS (ESI):calcd. for $\mathrm{C}_{19} \mathrm{H}_{21} \mathrm{~N}_{3} \mathrm{NaO}\left([\mathrm{M}+\mathrm{Na}]^{+}\right)$: 330.1582 , found: 330.1581 .

\section{4-(2-(4-Chlorophenyl)propan-2-yl)-2,6-dimethyl-1,4-dihydropyridine-3,5-dicarbonitrile(7c)}

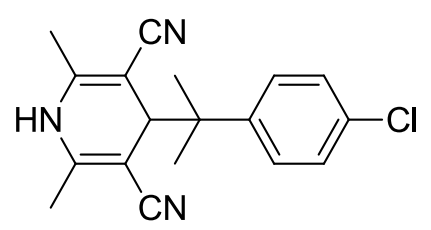

With general procedure $\mathbf{E}$, reaction of 2-(4-chlorophenyl)-2-methylpropanal $(10 \mathrm{mmol})$ and $\beta$-aminocrotonitrile $(20 \mathrm{mmol})$ provided the $7 \mathrm{c}$ after flash column chromatography (60\% EtOAc in petroleum ether) as a white solid (2.2 g, $71 \%) . \mathrm{R}_{f}=0.35$ (50 vol \% EtOAc/petroleum ether). M.P. 175-177 ${ }^{\circ} \mathrm{C}$. IR (neat) 3333, 2997, 2947, 2189, 1748, 1716, 1644, 1516, 1463, 1418, 
$1259 \mathrm{~cm}^{-1},{ }^{1} \mathrm{H}$ NMR $\left(400 \mathrm{MHz}, \mathrm{CDCl}_{3}\right.$, TMS) $\delta 7.24(\mathrm{~s}, 4 \mathrm{H}), 6.80(\mathrm{~s}, 1 \mathrm{H}), 3.25(\mathrm{~s}, 1 \mathrm{H}), 2.01(\mathrm{~s}, 6 \mathrm{H})$, 1.38 (s, 6H). ${ }^{13} \mathrm{C}$ NMR $\left(100 \mathrm{MHz}, \mathrm{CDCl}_{3}\right) \delta 149.2,143.0,132.7,128.5,127.8,119.6,80.3,47.4,46.4$, 24.5, 18.1. HRMS (ESI):calcd. for $\mathrm{C}_{18} \mathrm{H}_{17} \mathrm{ClN}_{3}\left([\mathrm{M}-\mathrm{H}]^{+}\right)$: 310.1111 , found: 310.1113 .

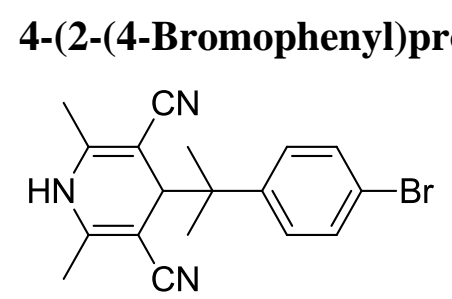

pan-2-yl)-2,6-dimethyl-1,4-dihydropyridine-3,5-dicarbonitrile(7d)

With general procedure $\mathbf{E}$, reaction of 2-(4-bromophenyl)-2-methylpropanal $(10 \mathrm{mmol})$ and $\beta$-aminocrotonitrile (20 mmol) provided $7 \mathbf{d}$ after flash column chromatography (60\% EtOAc in petroleum ether) as a white solid $(2.8 \mathrm{~g}, 80 \%) . \mathrm{R}_{f}=0.45(50 \mathrm{vol} \% \mathrm{EtOAc}$ in petroleum ether). M.P. $187-189^{\circ} \mathrm{C}$. IR (neat) $3143,2887,2857,2197$, 1746, 1679, 1646, 1507, 1463, 1422, 1393, $1275 \mathrm{~cm}^{-1},{ }^{1} \mathrm{H}$ NMR $(400 \mathrm{MHz}$, $\left.\mathrm{CDCl}_{3}, \mathrm{TMS}\right) \delta 7.39(\mathrm{~d}, J=8.5 \mathrm{~Hz}, 2 \mathrm{H}), 7.18(\mathrm{~d}, J=8.5 \mathrm{~Hz}, 2 \mathrm{H}), 6.78(\mathrm{~s}, 1 \mathrm{H}), 3.26(\mathrm{~s}, 1 \mathrm{H}), 2.01(\mathrm{~s}$, $6 \mathrm{H}), 1.37(\mathrm{~s}, 6 \mathrm{H}) .{ }^{13} \mathrm{C}$ NMR $\left(100 \mathrm{MHz}, \mathrm{CDCl}_{3}\right) \delta 149.2,143.5,130.7,128.9,120.8,119.6,80.3,47.4$, 46.5, 24.5, 18.2. HRMS (ESI):calcd. for $\mathrm{C}_{18} \mathrm{H}_{17} \mathrm{BrN}_{3}\left([\mathrm{M}-\mathrm{H}]^{+}\right)$: 354.0606 , found: 354.0606 .

2,6-Dimethyl-4-(2-(4-(trifluoromethyl)phenyl)propan-2-yl)-1,4-dihydropyridine-3,5-dicarbonitril e(7e)

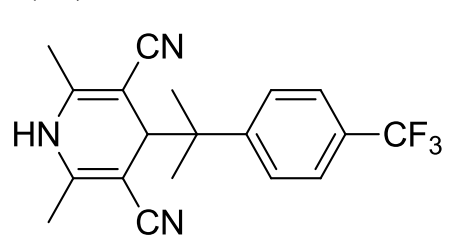

With general procedure $\mathbf{E}$, reaction of 2-methyl-2-(4-(trifluoromethyl)phenyl)propanal $\quad\left(\begin{array}{lll}10 & \mathrm{mmol})\end{array}\right.$ and $\beta$-aminocrotonitrile $(20 \mathrm{mmol})$ provided $7 \mathbf{e}$ after flash column chromatography (50\% EtOAc in petroleum ether) as a white solid $(1.7 \mathrm{~g}$, $50 \%) . \mathrm{R}_{f}=0.45$ (50 vol \% EtOAc/petroleum ether). M.P. 176-178 ${ }^{\circ} \mathrm{C} . \mathrm{IR}$ (neat) $3308,3226,2969,2202,1748,1679,1644,1514,1463,1328,1275$, $1161,1122,1063 \mathrm{~cm}^{-1},{ }^{1} \mathrm{H}$ NMR $\left(400 \mathrm{MHz}, \mathrm{CDCl}_{3}, \mathrm{TMS}\right) \delta 7.53(\mathrm{~d}, J=8.4 \mathrm{~Hz}, 2 \mathrm{H}), 7.44(\mathrm{~d}, J=8.4$ $\mathrm{Hz}, 2 \mathrm{H}), 6.55(\mathrm{~s}, 1 \mathrm{H}), 3.30(\mathrm{~s}, 1 \mathrm{H}), 1.99(\mathrm{~s}, 6 \mathrm{H}), 1.43(\mathrm{~s}, 6 \mathrm{H}) .{ }^{13} \mathrm{C}$ NMR $\left(100 \mathrm{MHz}, \mathrm{CDCl}_{3}\right) \delta 149.1$, 148.6, 127.4, 125.6, $124.5(\mathrm{q}, J=3.7 \mathrm{~Hz}), 80.5,47.4,46.9,24.5,18.1$. HRMS (ESI):calcd. for $\mathrm{C}_{19} \mathrm{H}_{17} \mathrm{~F}_{3} \mathrm{~N}_{3}\left([\mathrm{M}-\mathrm{H}]^{+}\right): 344.1375$, found: 344.1375 .

\section{4-(1-(4-Chlorophenyl)cyclopentyl)-2,6-dimethyl-1,4-dihydropyridine-3,5-dicarbonitrile(7f)}

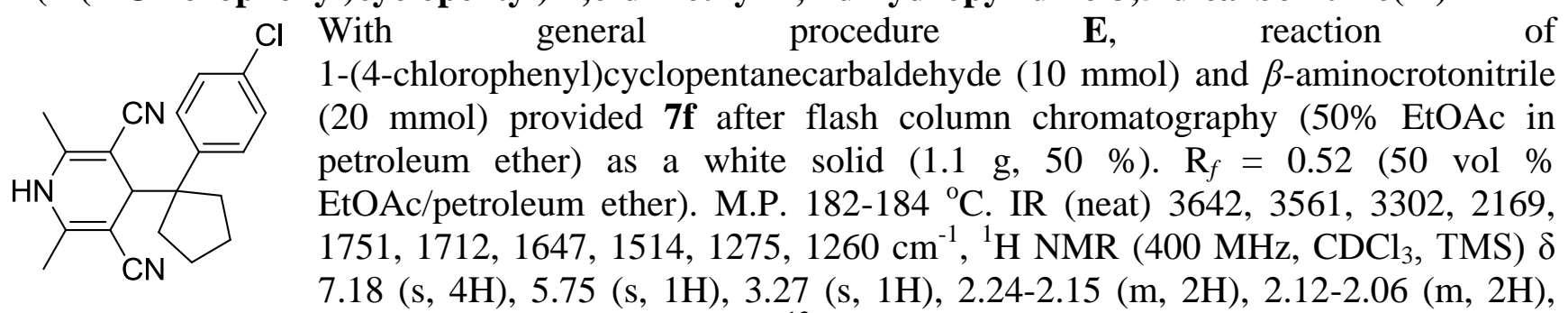
1.89 (s, 6H), 1.80-1.76 (m, 2H), 1.59-1.48 (m, 2H). ${ }^{13} \mathrm{C}$ NMR (100 MHz, $\left.\mathrm{CDCl}_{3}\right) \delta 148.6,140.3,132.6$, 129.6, 126.9, 120.1, 81.0, 60.1, 44.6, 35.2, 21.8, 18.2. HRMS (ESI):calcd. for $\mathrm{C}_{20} \mathrm{H}_{19} \mathrm{ClN}_{3}\left([\mathrm{M}-\mathrm{H}]^{-}\right)$: 336.1268 , found: 336.1267 .

\section{2,6-Dimethyl-4-(2-(thiophen-2-yl)propan-2-yl)-1,4-dihydropyridine-3,5-dicarbonitrile(7g)}

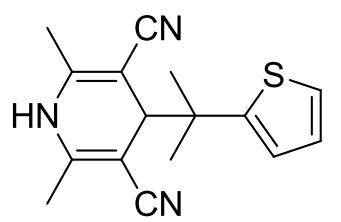

With general procedure $\mathbf{E}$, reaction of 2-methyl-2-(thiophen-2-yl)propanal (10 $\mathrm{mmol})$ and $\beta$-aminocrotonitrile $(20 \mathrm{mmol})$ provided $\mathbf{7 g}$ after flash column chromatography (50\% EtOAc in petroleum ether) as a white solid $(1.1 \mathrm{~g}, 50 \%)$. $\mathrm{R}_{f}=0.40$ (50 vol \% EtOAc in petroleum ether). M.P. $151-153{ }^{\circ} \mathrm{C}$. IR (neat) 3646 , $3310,2985,2188,1744,1683,1644,1514,1463,1436,1275,1260 \mathrm{~cm}^{-1},{ }^{1} \mathrm{H}$ NMR $\left(400 \mathrm{MHz}, \mathrm{CDCl}_{3}, \mathrm{TMS}\right) \delta 7.24(\mathrm{dd}, J=5.1,1.1 \mathrm{~Hz}, 1 \mathrm{H}), 6.94(\mathrm{dd}, J=5.1$, $3.6 \mathrm{~Hz}, 1 \mathrm{H}), 6.82(\mathrm{dd}, J=3.6,1.1 \mathrm{~Hz}, 1 \mathrm{H}), 6.26(\mathrm{~s}, 1 \mathrm{H}), 3.26(\mathrm{~s}, 1 \mathrm{H}), 2.07(\mathrm{~s}, 6 \mathrm{H}), 1.46(\mathrm{~s}, 6 \mathrm{H}) .{ }^{13} \mathrm{C}$ 
NMR (100 MHz, $\left.\mathrm{CDCl}_{3}\right) \delta 149.6,148.7,126.5,124.7,124.6,119.6,80.9,48.5,46.1,26.4,18.5$. HRMS (ESI):calcd. for $\mathrm{C}_{16} \mathrm{H}_{16} \mathrm{~N}_{3} \mathrm{~S}\left([\mathrm{M}-\mathrm{H}]^{+}\right)$: 282.1065 , found: 282.1070 .

\section{4-(2-(Benzofuran-2-yl)propan-2-yl)-2,6-dimethyl-1,4-dihydropyridine-3,5-dicarbonitrile(7h)}

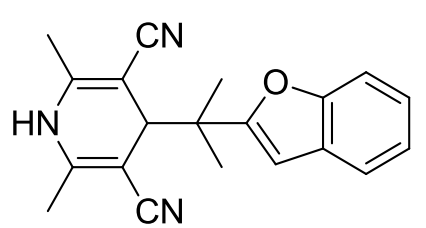

With general procedure $\mathbf{E}$, reaction of 2-(benzofuran-2-yl)-2-methylpropanal $(10 \mathrm{mmol})$ and $\beta$-aminocrotonitrile $(20 \mathrm{mmol})$ provided $\mathbf{7 h}$ after flash column chromatography $(50 \%$ EtOAc in petroleum ether) as a white solid (950 mg, $30 \%) . \mathrm{R}_{f}=0.50(50 \mathrm{vol} \%$ EtOAc in petroleum ether). M.P. $180-182^{\circ} \mathrm{C}$. IR (neat) $3291,3233,3114$, 2970, 2925, 2199, 1651, 1465, 1454, 1289, 1253, 1169, $752 \mathrm{~cm}^{-1},{ }^{1} \mathrm{H}$ NMR

(400 MHz, $\left.\mathrm{CDCl}_{3}, \mathrm{TMS}\right) \delta 7.49(\mathrm{~d}, J=7.2 \mathrm{~Hz}, 1 \mathrm{H}), 7.41(\mathrm{~d}, J=7.2 \mathrm{~Hz}, 1 \mathrm{H}), 7.24-7.17(\mathrm{~m}, 2 \mathrm{H}), 6.76$ $(\mathrm{s}, 1 \mathrm{H}), 6.44(\mathrm{~s}, 1 \mathrm{H}), 3.60(\mathrm{~s}, 1 \mathrm{H}), 1.96(\mathrm{~s}, 6 \mathrm{H}), 1.39(\mathrm{~s}, 6 \mathrm{H}) .{ }^{13} \mathrm{C} \mathrm{NMR}\left(100 \mathrm{MHz}, \mathrm{CDCl}_{3}\right) \delta 161.6$, $154.8,149.1,128.4,123.8,122.5,120.5,119.3,110.9,103.4,80.2,45.1,44.5,22.7,18.2$. HRMS (ESI):calcd. for $\mathrm{C}_{20} \mathrm{H}_{18} \mathrm{~N}_{3} \mathrm{O}$ ([M-H]'): 316.1450 , found: 316.1449 .

\section{4-Benzhydryl-2,6-dimethyl-1,4-dihydropyridine-3,5-dicarbonitrile(7i)}

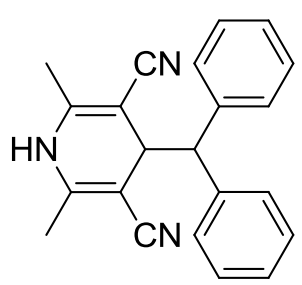

With general procedure $\mathbf{E}$, reaction of 2,2-diphenylacetaldehyde $(10 \mathrm{mmol})$ and $\beta$-aminocrotonitrile $(20 \mathrm{mmol})$ provided $7 \mathbf{i}$ after flash column chromatography $(50 \%$ EtOAc in petroleum ether) as a white solid ( $1.9 \mathrm{~g}, 60 \%)$. $\mathrm{R}_{f}=0.50(50 \mathrm{vol} \%$ EtOAc in petroleum ether). M.P. $168-170{ }^{\circ} \mathrm{C}$. IR (neat) $3669,3642,3302,2199$, $1742,1710,1677,1514,1418,1275,1253 \mathrm{~cm}^{-1},{ }^{1} \mathrm{H}$ NMR (400 MHz, $\left.\mathrm{CDCl}_{3}, \mathrm{TMS}\right)$ $\delta 7.40-7.26(\mathrm{~m}, 10 \mathrm{H}), 6.04(\mathrm{~s}, 1 \mathrm{H}), 4.10(\mathrm{~d}, J=6.8 \mathrm{~Hz}, 1 \mathrm{H}), 4.05(\mathrm{~d}, J=6.8 \mathrm{~Hz}$, $1 \mathrm{H}), 1.99(\mathrm{~s}, 6 \mathrm{H}) .{ }^{13} \mathrm{C}$ NMR $\left(100 \mathrm{MHz}, \mathrm{CDCl}_{3}\right) \delta 147.5,139.3,129.3,128.3,127.2$, 118.5, 83.1, 58.2, 41.0, 18.5. HRMS (ESI):calcd. for $\mathrm{C}_{22} \mathrm{H}_{18} \mathrm{~N}_{3}([\mathrm{M}-\mathrm{H}]$ ) $)$ :324.1501, found: 324.1502 .

\section{2,6-dimethyl-4-(2-phenylbutan-2-yl)-1,4-dihydropyridine-3,5-dicarbonitrile (7j)}<smiles>CCC(C)(c1ccccc1)C(C(=O)C#N)C1=C(C)NC(C)=C(C)C1</smiles>

To a flask charged with 2-methyl-2-phenylbutanal ${ }^{[19]}(1.62 \mathrm{~g}, 10 \mathrm{mmol})$ and 3 -aminocrotononitrile $(1.64 \mathrm{~g}, 20 \mathrm{mmol})$ was added HOAc $(8 \mathrm{~mL})$. The mixture was heated at $110{ }^{\circ} \mathrm{C}$ for 1.5 hours. The achieved solution was poured into $30 \mathrm{~mL}$ water. The suspension was extracted with EtOAc three times. The combined organic layers were washed with water and saturated aqueous solution of $\mathrm{NaHCO}_{3}$ until no HOAc residue. The solution was dried $\left(\mathrm{Na}_{2} \mathrm{SO}_{4}\right)$ and concentrated. The residue was washed with petroleum ether/EtOAc $(9: 1, \mathrm{v} / \mathrm{v})$ to give a pale yellow powder. The compound did not survive the column chromatography. So the compound is not as pure as other analogues but good enough for the photoredox reaction. IR (neat): 3293, 3231, 3110, 2972, 2197, 1769, $1688,1245,699,498 .{ }^{1} \mathrm{H}$ NMR $\left(400 \mathrm{MHz}, \mathrm{CDCl}_{3}, \mathrm{TMS}\right) \delta$ 7.30-7.25 (m, 5H), $6.29(\mathrm{~s}, 1 \mathrm{H}), 3.31(\mathrm{~s}$, $1 \mathrm{H}), 2.23-2.16(\mathrm{~m}, 1 \mathrm{H}), 2.04(\mathrm{~s}, 3 \mathrm{H}), 2.00(\mathrm{~s}, 3 \mathrm{H}), 1.82-1.74(\mathrm{~m}, 1 \mathrm{H}), 1.34(\mathrm{~s}, 3 \mathrm{H}), 0.74(\mathrm{t}, J=6 \mathrm{~Hz}$, $3 \mathrm{H}) .{ }^{13} \mathrm{C}$ NMR $\left(100 \mathrm{MHz}, \mathrm{CDCl}_{3}\right) \delta 148.5,148.2,128.5,127.9,127.6,126.6,125.8,120.4,119.1$, 116.7, 81.4, 80.9, 50.7, 47.9, 29.6, 19.2, 18.5, 18.4, 8.3. HRMS (EI): calcd. for $\mathrm{C}_{19} \mathrm{H}_{21} \mathrm{~N}_{3}\left([\mathrm{M}]^{+}\right)$: 291.1736, found: 291.1742 .

\section{2,2,3-Trimethyl-1,3-diphenylbutan-1-one (8a)}




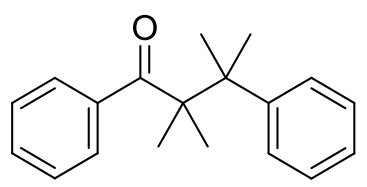

With general procedure $\mathbf{G}$, reaction of $\mathbf{1 a}(0.2 \mathrm{mmol})$ and $\mathbf{7 a}(0.6 \mathrm{mmol})$ provided the product $8 \mathbf{a}$ after flash column chromatography $(2.0$ vol \% EtOAc in petroleum ether) as a colorless oil $(21.3 \mathrm{mg}, 40 \%) . \mathrm{R}_{f}=0.50(5.0 \mathrm{vol} \%$ EtOAc in petroleum ether). IR (neat), 3006, 2989, 2865, 1681, 1646, 1454, $1275,1260,1054,1032,1013,767,749,704 \mathrm{~cm}^{-1} .{ }^{1} \mathrm{H}$ NMR $\left(400 \mathrm{MHz}, \mathrm{CDCl}_{3}\right.$, TMS) $\delta$ 7.42-7.39 (m, 2H), 7.30-7.27 (m, 2H), 7.26-7.20 (m, 2H), 7.14-7.10 (m, 2H), 6.65-6.60 (m, $2 \mathrm{H}), 1.56(\mathrm{~s}, 6 \mathrm{H}), 1.23(\mathrm{~s}, 6 \mathrm{H}) .{ }^{13} \mathrm{C} \mathrm{NMR}\left(100 \mathrm{MHz}, \mathrm{CDCl}_{3}\right) \delta 212.4,146.8,141.5,129.8,128.3,127.9$, 127.4, 127.0, 126.2, 53.5, 43.4, 25.0, 24.4. HRMS (EI): calcd. for $\mathrm{C}_{19} \mathrm{H}_{21} \mathrm{O}\left([\mathrm{M}-\mathrm{H}]^{+}\right): 265.1592$, found: 265.1600. The HRMS (EI) showed an unusual peak of [M-H], so the original report was attached.

\section{3-(4-Methoxyphenyl)-2,2,3-trimethyl-1-phenylbutan-1-one (8b)}

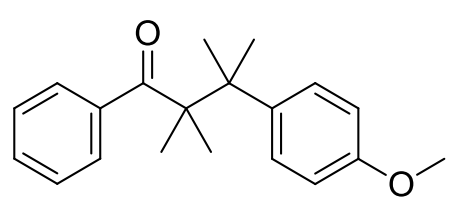

With general procedure $\mathbf{G}$, reaction of $\mathbf{1 a}(0.2 \mathrm{mmol})$ and $\mathbf{7 b}(0.6 \mathrm{mmol})$ provided the product $\mathbf{8 b}$ after flash column chromatography $(2.0 \mathrm{vol} \%$ EtOAc in petroleum ether) as a colorless oil $(20.7 \mathrm{mg}, 35 \%) . \mathrm{R}_{f}=0.40$ (5.0 vol \% EtOAc in petroleum ether). IR (neat), 3003, 2981, 2924, 2861, $2843,1454,1275,1260,1055,1032,1012,763,750 \mathrm{~cm}^{-1} .{ }^{1} \mathrm{H}$ NMR $(400$ $\left.\mathrm{MHz}, \mathrm{CDCl}_{3}, \mathrm{TMS}\right) \delta 7.31(\mathrm{~d}, J=7.7 \mathrm{~Hz}, 3 \mathrm{H}), 7.15(\mathrm{t}, J=7.7 \mathrm{~Hz}, 2 \mathrm{H}), 6.82(\mathrm{~d}, J=8.0 \mathrm{~Hz}, 2 \mathrm{H}), 6.71$ $(\mathrm{d}, J=8.0 \mathrm{~Hz}, 2 \mathrm{H}), 3.78(\mathrm{~s}, 3 \mathrm{H}), 1.52(\mathrm{~s}, 6 \mathrm{H}), 1.22(\mathrm{~s}, 6 \mathrm{H}) .{ }^{13} \mathrm{C} \mathrm{NMR}\left(100 \mathrm{MHz}, \mathrm{CDCl}_{3}\right) \delta 212.4$, 158.0, 141.6, 139.0, 129.8, 129.3, 127.5, 127.1, 113.2, 55.3, 53.6, 42.7, 25.2, 24.4. HRMS (EI): calcd. for $\mathrm{C}_{20} \mathrm{H}_{24} \mathrm{O}_{2}([\mathrm{M}]): 296.1776$, found: 296.1768 .

\section{3-(4-Chlorophenyl)-2,2,3-trimethyl-1-phenylbutan-1-one (8c)}

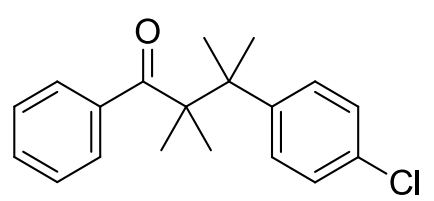

With general procedure $\mathbf{G}$, reaction of $\mathbf{1 a}(0.2 \mathrm{mmol})$ and $\mathbf{7 c}(0.6 \mathrm{mmol})$ provided the product 8c after flash column chromatography $(2.0$ vol \% EtOAc in petroleum ether) as a colorless oil $(28.8 \mathrm{mg}, 48 \%)$. $\mathrm{R}_{f}=0.40(5.0$ vol \% EtOAc in petroleum ether). IR (neat), 3002, 2981, 2920, 2865, 2842, $1471,1454,1275,1260,1054,1032,1012,763,750,702 \mathrm{~cm}^{-1} .{ }^{1} \mathrm{H}$ NMR (400 MHz, $\mathrm{CDCl}_{3}$, TMS) $\delta$ 7.33-7.29 (m, 3H), 7.26-7.22 (m, 2H), 7.21-7.17 (m, 2H), 6.81-6.76 (m, $2 \mathrm{H}), 1.53(\mathrm{~s}, 6 \mathrm{H}), 1.23(\mathrm{~s}, 6 \mathrm{H}) .{ }^{13} \mathrm{C} \mathrm{NMR}\left(100 \mathrm{MHz}, \mathrm{CDCl}_{3}\right) \delta 211.8,145.3,141.4,132.2,130.0,129.7$, 127.8, 127.6, 127.0, 53.4, 43.2, 25.1, 24.4. HRMS (EI): calcd. for $\mathrm{C}_{19} \mathrm{H}_{21} \mathrm{ClO}\left([\mathrm{M}]^{+}\right)$: 300.1281 , found: 300.1279 .

\section{3-(4-Bromophenyl)-2,2,3-trimethyl-1-phenylbutan-1-one (8d)}

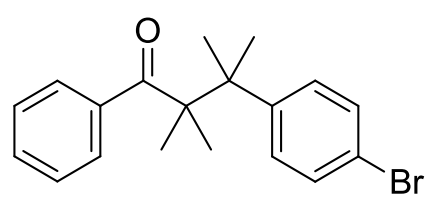

With general procedure $\mathbf{G}$, reaction of $\mathbf{1 a}(0.2 \mathrm{mmol})$ and $\mathbf{7 d}(0.6 \mathrm{mmol})$ provided the product 8d after flash column chromatography (2.0 vol \% EtOAc in petroleum ether) as a colorless oil ( $34.4 \mathrm{mg}, 50 \%)$. $\mathrm{R}_{f}=0.40(5.0$ vol \% EtOAc in petroleum ether). IR (neat), 3010, 2981, 2920, 2863, 2840, $1681,1642,1467,1348,1275,1260,1055,1032,1012,750,706 \mathrm{~cm}^{-1} .{ }^{1} \mathrm{H}$ NMR (400 MHz, CDCl 3 , TMS) $\delta 7.40(\mathrm{~d}, J=8.0 \mathrm{~Hz}, 2 \mathrm{H}), 7.31(\mathrm{t}, J=8.0 \mathrm{~Hz}, 1 \mathrm{H}), 7.25(\mathrm{~d}, J=8.0 \mathrm{~Hz}$, $2 \mathrm{H}), 7.19(\mathrm{t}, J=8.0 \mathrm{~Hz}, 2 \mathrm{H}), 6.79(\mathrm{~d}, J=8.0 \mathrm{~Hz}, 2 \mathrm{H}), 1.52(\mathrm{~s}, 6 \mathrm{H}), 1.22(\mathrm{~s}, 6 \mathrm{H}) .{ }^{13} \mathrm{C} \mathrm{NMR}(100 \mathrm{MHz}$, $\left.\mathrm{CDCl}_{3}\right) \delta 211.8,145.8,141.4,130.8,130.2,130.0,127.6,127.0,120.3,53.4,43.2,25.1$, 24.4.HRMS (EI): calcd. for $\mathrm{C}_{19} \mathrm{H}_{21} \mathrm{BrO}\left([\mathrm{M}]^{+}\right)$: 344.0776 , found: 344.0774 .

\section{2,2,3-Trimethyl-1-phenyl-3-(4-(trifluoromethyl)phenyl)butan-1-one (8e)}

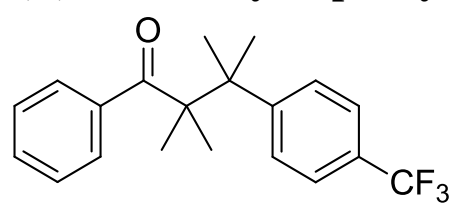

With general procedure $\mathbf{G}$, reaction of $\mathbf{1 a}(0.2 \mathrm{mmol})$ and $7 \mathbf{e}(0.6 \mathrm{mmol})$ provided the product $8 \mathbf{e}$ after flash column chromatography $(2.0 \mathrm{vol} \%$ EtOAc in petroleum ether) as a colorless oil $(25.4 \mathrm{mg}, 38 \%) . \mathrm{R}_{f}=0.30$ (5.0 vol \% EtOAc in petroleum ether). IR (neat), 3004, 2980, 2922, 2863, 
$1671,1472,1454,1330,1275,1055,1032,1012,765,750 \mathrm{~cm}^{-1} .{ }^{1} \mathrm{H}$ NMR $\left(400 \mathrm{MHz}, \mathrm{CDCl}_{3}, \mathrm{TMS}\right) \delta$ $7.53(\mathrm{~d}, J=24.0 \mathrm{~Hz}, 2 \mathrm{H}), 7.51(\mathrm{~d}, J=24.0 \mathrm{~Hz}, 2 \mathrm{H}), 7.32-7.28(\mathrm{~m}, 1 \mathrm{H}), 7.18-7.14(\mathrm{~m}, 2 \mathrm{H}), 6.75-6.73$ $(\mathrm{m}, 2 \mathrm{H}), 1.57(\mathrm{~s}, 6 \mathrm{H}), 1.25(\mathrm{~s}, 6 \mathrm{H}) .{ }^{13} \mathrm{C} \mathrm{NMR}\left(100 \mathrm{MHz}, \mathrm{CDCl}_{3}\right) \delta 211.4,150.9,141.2,130.1,128.7$, 128.7, 128.4, 127.6, 127.0, 124.6 (q, $J=4 \mathrm{~Hz}$ ), 53.5, 43.6, 25.0, 24.3. HRMS (EI): calcd. for $\mathrm{C}_{15} \mathrm{H}_{16} \mathrm{ClOS}([\mathrm{M}]): 334.1545$, found: 334.1539 .

\section{2-(1-(4-Chlorophenyl)cyclopentyl)-2-methyl-1-phenylpropan-1-one (8f)}

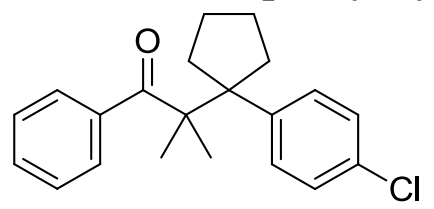

With general procedure $\mathbf{G}$, reaction of $\mathbf{1 a}(0.2 \mathrm{mmol})$ and the $\mathbf{7 f}(0.6 \mathrm{mmol})$ provided the product 8 f after flash column chromatography (2.0 vol \% EtOAc in petroleum ether) as a colorless oil (30.6 mg, 47\%). $\mathrm{R}_{f}=0.43(5.0$ vol \% EtOAc in petroleum ether). IR (neat), 2981, 2946, 1916, 2863, 1661, $1646,1471,1454,1275,1260,1054,1032,1013,765,750 \mathrm{~cm}^{-1} .{ }^{1} \mathrm{H}$ NMR (400 MHz, $\left.\mathrm{CDCl}_{3}, \mathrm{TMS}\right) \delta 7.30(\mathrm{~d}, J=8.0 \mathrm{~Hz}, 2 \mathrm{H}), 7.25-7.18(\mathrm{~m}, 3 \mathrm{H}), 7.11(\mathrm{t}, J=8.0 \mathrm{~Hz}, 2 \mathrm{H}), 6.65$ $(\mathrm{d}, J=8.0 \mathrm{~Hz}, 2 \mathrm{H}), 2.36-2.29(\mathrm{~m}, 2 \mathrm{H}), 1.96-1.89(\mathrm{~m}, 2 \mathrm{H}), 1.59-1.53(\mathrm{~m}, 2 \mathrm{H}), 1.22-1.15(\mathrm{~m}, 8 \mathrm{H}) .{ }^{13} \mathrm{C}$ NMR $\left(100 \mathrm{MHz}, \mathrm{CDCl}_{3}\right) \delta 212.7,141.5,141.3,132.3,130.6,129.9,128.1,127.6,126.6,57.1,53.1$, 32.8, 25.1, 21.8. HRMS (EI): calcd. for $\mathrm{C}_{15} \mathrm{H}_{16} \mathrm{ClOS}\left([\mathrm{M}]^{+}\right)$: 326.1437 , found: 326.1441 .

\section{2,2,3-Trimethyl-1-phenyl-3-(thiophen-2-yl)butan-1-one (8g)}

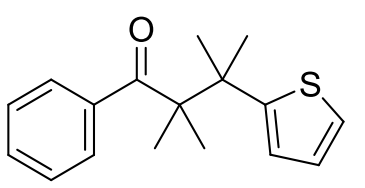

With general procedure $\mathbf{G}$, reaction of $1 \mathbf{a}(0.2 \mathrm{mmol})$ and $\mathbf{7 g}(0.6 \mathrm{mmol})$ provided the product $\mathbf{8 g}$ after flash column chromatography $(2.0 \mathrm{vol} \%$ EtOAc in petroleum ether) as a colorless oil $(24.5 \mathrm{mg}, 45 \%) . \mathrm{R}_{f}=0.45(5.0 \mathrm{vol} \%$ EtOAc in petroleum ether). IR (neat), 2980, 2843, 1661, 1646, 1473, 1454, $1275,1260,1054,1032,1014,763,750 \mathrm{~cm}^{-1} .{ }^{1} \mathrm{H} \mathrm{NMR}\left(400 \mathrm{MHz}, \mathrm{CDCl}_{3}\right.$, TMS) $\delta 7.32-7.28(\mathrm{~m}, 1 \mathrm{H}), 7.22-7.14(\mathrm{~m}, 3 \mathrm{H}), 6.95-6.92(\mathrm{~m}, 1 \mathrm{H}), 6.89-6.89(\mathrm{~m}, 1 \mathrm{H}), 6.82-6.76(\mathrm{~m}$, $2 \mathrm{H}), 1.58(\mathrm{~s}, 6 \mathrm{H}), 1.30(\mathrm{~s}, 6 \mathrm{H}) .{ }^{13} \mathrm{C} \mathrm{NMR}\left(100 \mathrm{MHz}, \mathrm{CDCl}_{3}\right) \delta 212.1,152.3,141.6,129.8,127.6,126.8$, 126.6, 125.7, 123.4, 53.6, 43.0, 26.7, 24.5. HRMS (EI): calcd. for $\mathrm{C}_{17} \mathrm{H}_{20} \mathrm{OS}\left([\mathrm{M}]^{+}\right): 272.1235$, found: 272.1240 .

\section{3-(Benzofuran-2-yl)-2,2,3-trimethyl-1-phenylbutan-1-one (8h)}

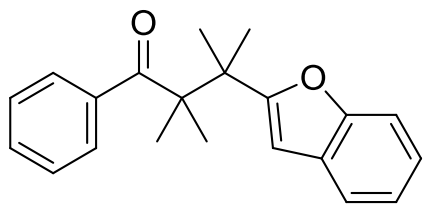

With general procedure $\mathbf{G}$, reaction of $\mathbf{1 a}(0.2 \mathrm{mmol})$ and the $\mathbf{7 h}(0.6 \mathrm{mmol})$ provided the product $\mathbf{8 h}$ after flash column chromatography (2.0 vol \% EtOAc in petroleum ether) as a colorless oil (21.5 mg, 40\%). $\mathrm{R}_{f}=0.50(5.0$ vol \% EtOAc in petroleum ether). IR (neat), 2981, 2925, 2853, 1668, 1596, 1454, 1369, 1256, 1199, 1168, 963, 750, $701 \mathrm{~cm}^{-1}$. ${ }^{1} \mathrm{H}$ NMR (400 MHz, $\left.\mathrm{CDCl}_{3}, \mathrm{TMS}\right) \delta$ 7.42-7.39 (m, 1H), 7.27-7.20 (m, 2H), 7.15-7.02 (m, 6H), $6.40(\mathrm{~s}, 1 \mathrm{H}), 1.47(\mathrm{~s}, 6 \mathrm{H})$, $1.29(\mathrm{~s}, 6 \mathrm{H}) .{ }^{13} \mathrm{C}$ NMR $\left(100 \mathrm{MHz}, \mathrm{CDCl}_{3}\right) \delta 210.6,164.0,154.2,141.3,129.9,128.4,127.6,127.2$, 123.5, 122.5, 120.5, 111.0, 103.2, 53.1, 42.3, 24.4, 23.7. HRMS (EI): calcd. for $\mathrm{C}_{21} \mathrm{H}_{22} \mathrm{O}_{2}\left([\mathrm{M}]^{+}\right)$: 306.1620, found: 306.1617 .

\section{3-(4-Chlorophenyl)-2,2,3-trimethyl-1-(thiophen-3-yl)butan-1-one (8i)}

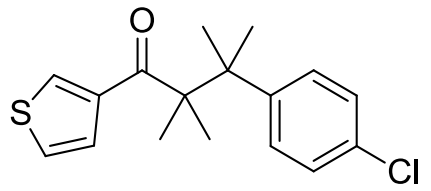

With general procedure $\mathbf{G}$, reaction of $\mathbf{1 p}(0.2 \mathrm{mmol})$ and $\mathbf{7 c}(0.6 \mathrm{mmol})$ provided the product $8 \mathbf{i}$ after flash column chromatography $(2.0$ vol \% EtOAc in petroleum ether) as a colorless oil (31.8 mg, 52\%). $\mathrm{R}_{f}=0.67$ (5.0 vol \% EtOAc in petroleum ether). IR (neat), 2980, 2924, 2861, 2838, 1747,

$1683,1646,1555,1471,1275,1260,1054,1032,1013,765,750 \mathrm{~cm}^{-1} .{ }^{1} \mathrm{H}$ NMR (400 MHz, $\left.\mathrm{CDCl}_{3}, \mathrm{TMS}\right) \delta 7.24(\mathrm{~d}, J=8.8 \mathrm{~Hz}, 2 \mathrm{H}), 7.17(\mathrm{~d}, J=8.8 \mathrm{~Hz}, 2 \mathrm{H}), 7.17(\mathrm{dd}, J=2.9$, $1.2 \mathrm{~Hz}, 1 \mathrm{H}), 7.07(\mathrm{dd}, J=5.1,2.9 \mathrm{~Hz}, 1 \mathrm{H}), 6.92(\mathrm{dd}, J=5.1,1.2 \mathrm{~Hz}, 1 \mathrm{H}), 1.45(\mathrm{~s}, 6 \mathrm{H}), 1.29(\mathrm{~s}, 6 \mathrm{H})$. 
${ }^{13} \mathrm{C}$ NMR $\left(100 \mathrm{MHz}, \mathrm{CDCl}_{3}\right) \delta 202.6,144.8,142.8,132.2,130.1,129.6,128.5,127.6,124.2,53.5$, 43.0, 25.0, 23.6. HRMS (EI): calcd. for $\mathrm{C}_{17} \mathrm{H}_{19} \mathrm{ClOS}\left([\mathrm{M}]^{+}\right)$: 306.0845 , found: 306.0840 .

\section{3-(4-Bromophenyl)-2,2,3-trimethyl-1-(thiophen-3-yl)butan-1-one $(8 \mathbf{j})$}

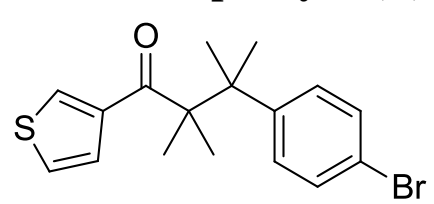

With general procedure $\mathbf{G}$, reaction of $\mathbf{1 p}(0.2 \mathrm{mmol})$ and $\mathbf{7 d}(0.6 \mathrm{mmol})$ provided the product $\mathbf{8 j}$ after flash column chromatography (2.0 vol \% EtOAc in petroleum ether) as a colorless oil ( $35.1 \mathrm{mg}, 50 \%)$. $\mathrm{R}_{f}=0.70(5.0$ vol \% EtOAc in petroleum ether). IR (neat), 2980, 2918, 2867, 1746, 1646, $1555,1469,1448,1397,1275,1260,1054,1032,1013,765,749,702 \mathrm{~cm}^{-1}$.

${ }^{1} \mathrm{H}$ NMR $\left(400 \mathrm{MHz}, \mathrm{CDCl}_{3}\right.$, TMS) $\delta 7.33(\mathrm{~d}, J=8.6 \mathrm{~Hz}, 2 \mathrm{H}), 7.18(\mathrm{~d}, J=8.6 \mathrm{~Hz}, 2 \mathrm{H}), 7.17(\mathrm{dd}, J=$ $2.9,0.8 \mathrm{~Hz}, 3 \mathrm{H}), 7.08(\mathrm{dd}, J=5.1,2.9 \mathrm{~Hz}, 1 \mathrm{H}), 6.94(\mathrm{dd}, J=5.1,0.8 \mathrm{~Hz}, 1 \mathrm{H}), 1.44(\mathrm{~s}, 6 \mathrm{H}), 1.29(\mathrm{~s}$, $6 \mathrm{H}) .{ }^{13} \mathrm{C}$ NMR $\left(100 \mathrm{MHz}, \mathrm{CDCl}_{3}\right) \delta 202.5,145.4,142.8,130.5,130.2,130.0,128.6,124.2,120.3,53.5$, 42.0, 24.9, 23.6. HRMS (EI): calcd. for $\mathrm{C}_{15} \mathrm{H}_{16} \mathrm{BrOS}\left([\mathrm{M}]^{+}\right)$: 350.0340 , found: 350.0334 .

\section{2,2,3-Trimethyl-1,3-di(thiophen-2-yl)butan-1-one (8k)}

With general procedure $\mathbf{G}$, reaction of $10(0.2 \mathrm{mmol})$ and $7 \mathrm{~g}(0.6 \mathrm{mmol})$

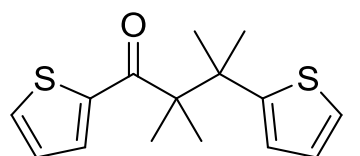
provided the product $\mathbf{8 k}$ after flash column chromatography $(2.0 \mathrm{vol} \%$ EtOAc in petroleum ether) as a colorless oil $(16.7 \mathrm{mg}, 30 \%) . \mathrm{R}_{f}=0.62(5.0 \mathrm{vol} \%$ EtOAc in petroleum ether). IR (neat), 2981, 2867, 2844, 1467, 1457, 1275, $1260,1055,1032,1012,767,750,700 \mathrm{~cm}^{-1} .{ }^{1} \mathrm{H}$ NMR $\left(400 \mathrm{MHz}, \mathrm{CDCl}_{3}, \mathrm{TMS}\right) \delta 7.44(\mathrm{dd}, J=5.0$, $0.8 \mathrm{~Hz}, 1 \mathrm{H}), 7.16(\mathrm{dd}, J=4.0,0.8 \mathrm{~Hz}, 1 \mathrm{H}), 7.07(\mathrm{dd}, J=5.0,1.1 \mathrm{~Hz}, 1 \mathrm{H}), 6.89(\mathrm{dd}, J=5.0,4.0 \mathrm{~Hz}$, $1 \mathrm{H}), 6.83(\mathrm{dd}, J=5.0,3.6 \mathrm{~Hz}, 1 \mathrm{H}), 6.80(\mathrm{dd}, J=3.6,1.1 \mathrm{~Hz}, 1 \mathrm{H}), 1.50(\mathrm{~s}, 6 \mathrm{H}), 1.44(\mathrm{~s}, 6 \mathrm{H}) .{ }^{13} \mathrm{C} \mathrm{NMR}$ $\left(100 \mathrm{MHz}, \mathrm{CDCl}_{3}\right) \delta 199.0,151.5,146.0,132.4,131.5,127.5,126.2,125.6,123.2,53.8,42.8,26.6$, 23.8. HRMS (EI): calcd. for $\mathrm{C}_{15} \mathrm{H}_{18} \mathrm{OS}_{2}\left([\mathrm{M}]^{+}\right): 278.0799$, found: 278.0803 .

\section{2,2,3-Trimethyl-3-(thiophen-2-yl)-1-(thiophen-3-yl)butan-1-one (8l)}

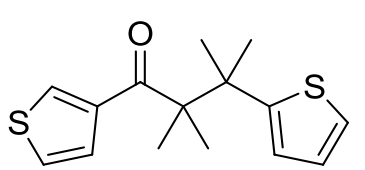

With general procedure $\mathbf{G}$, reaction of $\mathbf{1 p}(0.2 \mathrm{mmol})$ and $\mathbf{7 g}(0.6 \mathrm{mmol})$ provided the product $\mathbf{8} \mathbf{I}$ after flash column chromatography $(2.0$ vol $\%$ EtOAc in petroleum ether) as a colorless oil $(17.8 \mathrm{mg}, 32 \%) . \mathrm{R}_{f}=0.64(5.0 \mathrm{vol} \%$ EtOAc in petroleum ether). IR (neat), 2981, 2920, 2861, 2845, 1646, 1473, $1449,1275,1260,1055,1032,1013,759,749,706 \mathrm{~cm}^{-1} .{ }^{1} \mathrm{H}$ NMR $\left(400 \mathrm{MHz}, \mathrm{CDCl}_{3}, \mathrm{TMS}\right) \delta 7.19$ $(\mathrm{dd}, J=2.9,1.0 \mathrm{~Hz}, 1 \mathrm{H}), 7.11(\mathrm{dd}, J=5.1,1.0 \mathrm{~Hz}, 1 \mathrm{H}), 7.08(\mathrm{dd}, J=5.1,2.9 \mathrm{~Hz}, 1 \mathrm{H}), 6.95(\mathrm{dd}, J=$ $5.1,1.0 \mathrm{~Hz}, 1 \mathrm{H}), 6.87(\mathrm{dd}, J=5.1,3.6 \mathrm{~Hz}, 1 \mathrm{H}), 6.81(\mathrm{dd}, J=3.6,1.0 \mathrm{~Hz}, 1 \mathrm{H}), 1.51(\mathrm{~s}, 6 \mathrm{H}), 1.37(\mathrm{~s}$, $6 \mathrm{H}) .{ }^{13} \mathrm{C}$ NMR $\left(100 \mathrm{MHz}, \mathrm{CDCl}_{3}\right) \delta 203.4,151.9,143.0,129.6,128.5,126.5,125.6,124.0,123.3,53.6$, 42.7, 26.5, 23.9. HRMS (EI): calcd. for $\mathrm{C}_{15} \mathrm{H}_{18} \mathrm{OS}_{2}\left([\mathrm{M}]^{+}\right): 278.0799$, found: 278.0800 .

\section{2,2-Dimethyl-1,3,3-triphenylpropan-1-one (8m)}

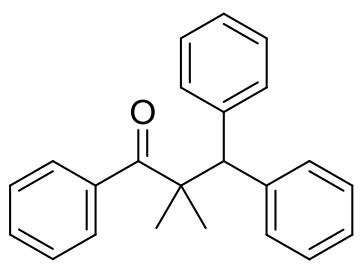

With general procedure $\mathbf{G}$, reaction of $\mathbf{1 a}(0.2 \mathrm{mmol})$ and $7 \mathbf{i}(0.6 \mathrm{mmol})$ provided the product $\mathbf{8 m}$ after flash column chromatography (2.0 vol \% EtOAc in petroleum ether) as a colorless oil $(36.4 \mathrm{mg}, 58 \%) . \mathrm{R}_{f}=0.52(5.0 \mathrm{vol} \%$ EtOAc in petroleum ether). IR (neat), 2981, 2918, 1916, 2867, 1661, 1645, $1553,1255,1032,1015,750,704 \mathrm{~cm}^{-1} .{ }^{1} \mathrm{H}$ NMR $\left(400 \mathrm{MHz}, \mathrm{CDCl}_{3}, \mathrm{TMS}\right) \delta$ 7.30-7.27 (m, 1H), 7.22-7.15 (m, 12H), 7.12-7.08 (m, 2H), $4.66(\mathrm{~s}, 1 \mathrm{H}), 1.34(\mathrm{~s}$, $6 \mathrm{H}) .{ }^{13} \mathrm{C}$ NMR $\left(100 \mathrm{MHz}, \mathrm{CDCl}_{3}\right) \delta 210.5,140.6,140.1,130.2,130.2,128.1$, 127.8, 127.1, 126.4, 58.7, 52.1, 25.6. HRMS (EI): calcd. for $\mathrm{C}_{23} \mathrm{H}_{22} \mathrm{O}\left([\mathrm{M}]^{+}\right)$: 314.1671, found: 314.1670. 


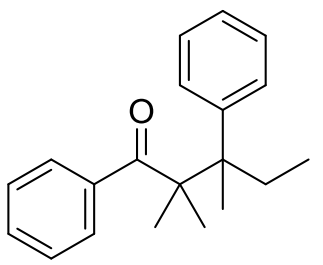

With general procedure $\mathbf{G}$, reaction of $\mathbf{1 a}(0.2 \mathrm{mmol})$ and $\mathbf{7} \mathbf{j}(0.6 \mathrm{mmol})$ provided the product $8 \mathbf{n}$ after flash column chromatography $(2.0 \mathrm{vol} \%$ EtOAc in petroleum ether) as a colorless oil $(39.2 \mathrm{mg}, 70 \%) . \mathrm{R}_{f}=0.52(5.0 \mathrm{vol} \%$ EtOAc in petroleum ether). IR: $3060,3006,2975,2929,1682,1596,1459,1387,1299,1168 .{ }^{1} \mathrm{H}$ NMR $\left(400 \mathrm{MHz}, \mathrm{CDCl}_{3}\right) \delta$ 7.33-7.20 (m, 6H), 7.11 (m, 2H), 6.67-6.58 (m, 2H), $2.52(\mathrm{~m}$, $1 \mathrm{H}), 1.76(\mathrm{~m}, 1 \mathrm{H}), 1.53(\mathrm{~s}, 3 \mathrm{H}), 1.24(\mathrm{~s}, 3 \mathrm{H}), 1.19(\mathrm{~s}, 3 \mathrm{H}), 0.72(\mathrm{t}, J=7.3 \mathrm{~Hz}, 3 \mathrm{H})$. ${ }^{13} \mathrm{C}$ NMR $\left(100 \mathrm{MHz}, \mathrm{CDCl}_{3}\right) \delta 212.5,143.5,141.6,129.7,129.2,127.9,127.4,127.0,126.1,54.4$, 47.0, 27.1, 24.4, 24.3, 20.6, 8.8. HRMS (EI): calcd. for $\mathrm{C}_{20} \mathrm{H}_{25} \mathrm{O}\left([\mathrm{M}+\mathrm{H}]^{+}\right)$: 281.1905, found: 281.1910. We found this compound give an unusual peak of $[\mathrm{M}+\mathrm{H}]^{+}$. So the HRMS was attached.

\section{Pent-4-en-1-yl 3-(4-chlorophenyl)-2,2,3-trimethylbutanoate (80)}

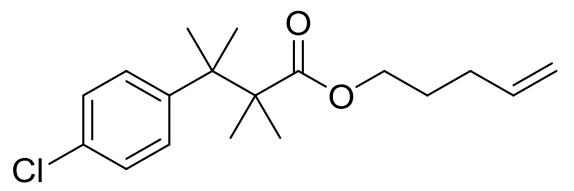

With general procedure $\mathbf{G}$, the $\mathbf{1} \mathbf{w}(0.2 \mathrm{mmol})$ and the $\mathbf{7 c}(0.6 \mathrm{mmol})$ provided the product 80 after flash column chromatography $(2.0$ vol \% EtOAc in petroleum ether) as a colorless oil $(21.5 \mathrm{mg}, 40 \%) . \mathrm{R}_{f}=0.52(1.0$ vol \% EtOAc in petroleum ether). IR: 2976, 2925, 2854, 1736, 1714, 1464, $1415,1275,1165,1111 .{ }^{1} \mathrm{H}$ NMR $\left(400 \mathrm{MHz}, \mathrm{CDCl}_{3}\right) \delta 7.28-7.24(\mathrm{~m}, 4 \mathrm{H})$, 5.84-5.74 (m, 1H), 5.07-4.95 (m, 2H), $3.96(\mathrm{t}, J=6.5 \mathrm{~Hz}, 2 \mathrm{H}), 2.07-2.02(\mathrm{~m}, 2 \mathrm{H}), 1.70-1.62(\mathrm{~m}, 2 \mathrm{H}), 1.44(\mathrm{~s}, 6 \mathrm{H})$, 1.11 (s, 6H). ${ }^{13} \mathrm{C}$ NMR $\left(100 \mathrm{MHz}, \mathrm{CDCl}_{3}\right) \delta 176.5,144.6,137.5,131.9,129.2,127.3,115.2,63.7,48.9,42.3,30.1$, 27.6, 24.9, 22.0. HRMS (EI): calcd. for $\mathrm{C}_{18} \mathrm{H}_{26} \mathrm{O}_{2} \mathrm{Cl}([\mathrm{M}+\mathrm{H}])$ : 309.1621. found: 309.1616. The HRMS (EI) showed an unusual peak of $[\mathrm{M}+\mathrm{H}]$, so the original report was attached.

[1] Chen J.-J., Guo W., Wang Z.-Y., Hu L., Chen F., Xia Y.-Z. J. Org. Chem. 2016, 81, 5504-5512.

[2] Liu, L; Kaib, P. S. J.; Tap, A; List, B. Med. Chem. 2014, 57, 3464-3483.

[3] Meyers, C. Y.; Roch, C.-Y.-K.; Hua, D. H.; Kolb, V. M.; Matthews, W. S.; Parady, T. E.; Horii, T.; Sandrock, P. B.; Hou, Y.; Xie, S. J. Org. Chem. 2003, 68, 500-511.

[4] Song S., Li X.-W., Sun X., Yuan Y.-Z., Jiao N. Green Chem., 2015, 17, 3285-3289.

[5] Liska, R. Heterocycles 2001, 55,1475-1486.

[6] Ou S., Cao C.-R., Jing M., Liu J.-T. Eur. J. Org. Chem. 2013, 6510-6513.

[7] Sharley, J. S.; Perez, A. M. C.; Ferri, E. E.; Miranda, A. F.; Baxendale, I. R. Tetrahedron 2016, 72, 2947-2954.

[8] Metlesics, W.; Wessely, F. Monatsh. Chem. 1957, 88, 108-117.

[9] Liu C., Liu D., Zhang W., Zhou L.-L., Lei A.-W. Org. Lett., 2013, 15, 6166-6169.

[10] Li G.-X., Chen R., Wu L., Fu Q.-Q., Zhang X.-M., Tang Z. Angew. Chem. Int. Ed. 2013, 52, 8432-8436.

[11] Li, Y.; Pouliot, M.; Vogler, T.; Renaud, P.; Studer, A. Org. Lett. 2012, 14, 4474-4477.

[12] Shaver, M. P.; Allan, L. E. N.; Gibson, V. C. Organometallics 2007, 26, 4725-4730.

[13] Johnston L. J., Tencer M., Scaiano J. C. J. Org. Chem. 1986, 51, 2806-2808.

[14] Cai X.-H., Yang H.-J., Zhang G.-L. Can. J. Chem. 2005, 83, 273-275.

[15] Pardo, C.; Charpentier-Morize, M. J. Chem. Soc., Chem. Commun. 1982, 1037-1039.

[16] Thomas R. Barbee, T. R.; Albizati, K. F. J. Org. Chem. 1991, 56, 6764-6773.

[17] Chen, X.; Liu, X.; Mohr, J. T. J. Am. Chem. Soc. 2016, 138, 6364-6367.

[18] Meshram, H. M.; Thakur, P. B.; Babu, B. M.; Bangade, V. M. Tetrahedron Letters 2012, 53, 1780-1785.

[19] James, B. R.; Young, C. G. J. Organomet. Chem. 1985, 285, 321-332. 
$15{ }^{1} \mathrm{H}$ NMR and ${ }^{13} \mathrm{C}$ NMR spectra

2-Bromo-1-(3-fluorophenyl)-2-methylpropan-1-one (1e)

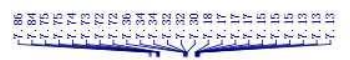

|| ||

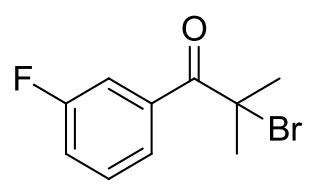

8

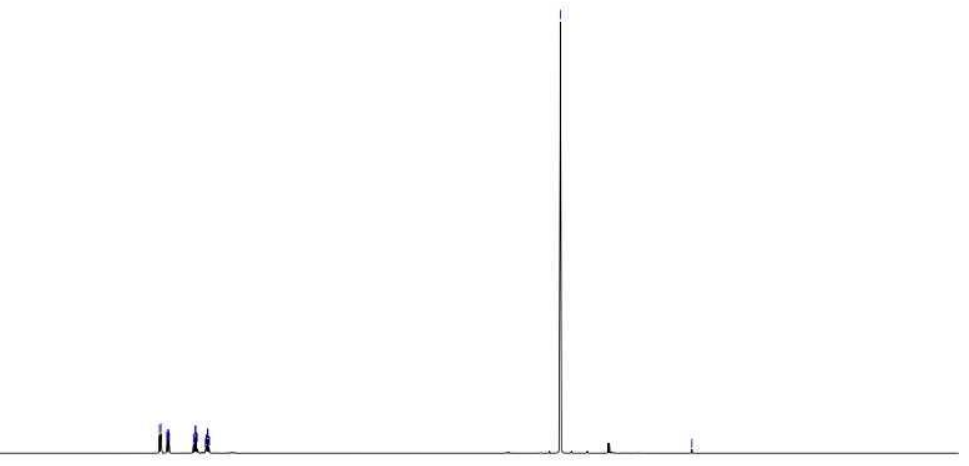

管鯙

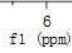

요용

章

$\stackrel{9}{\overline{1}}$

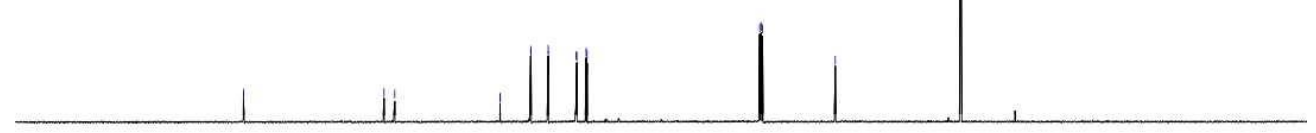

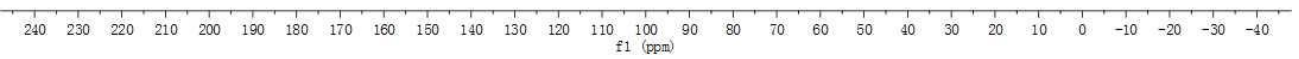

S46 
2-Bromo-1-(3-chlorophenyl)-2-methylpropan-1-one (1f)
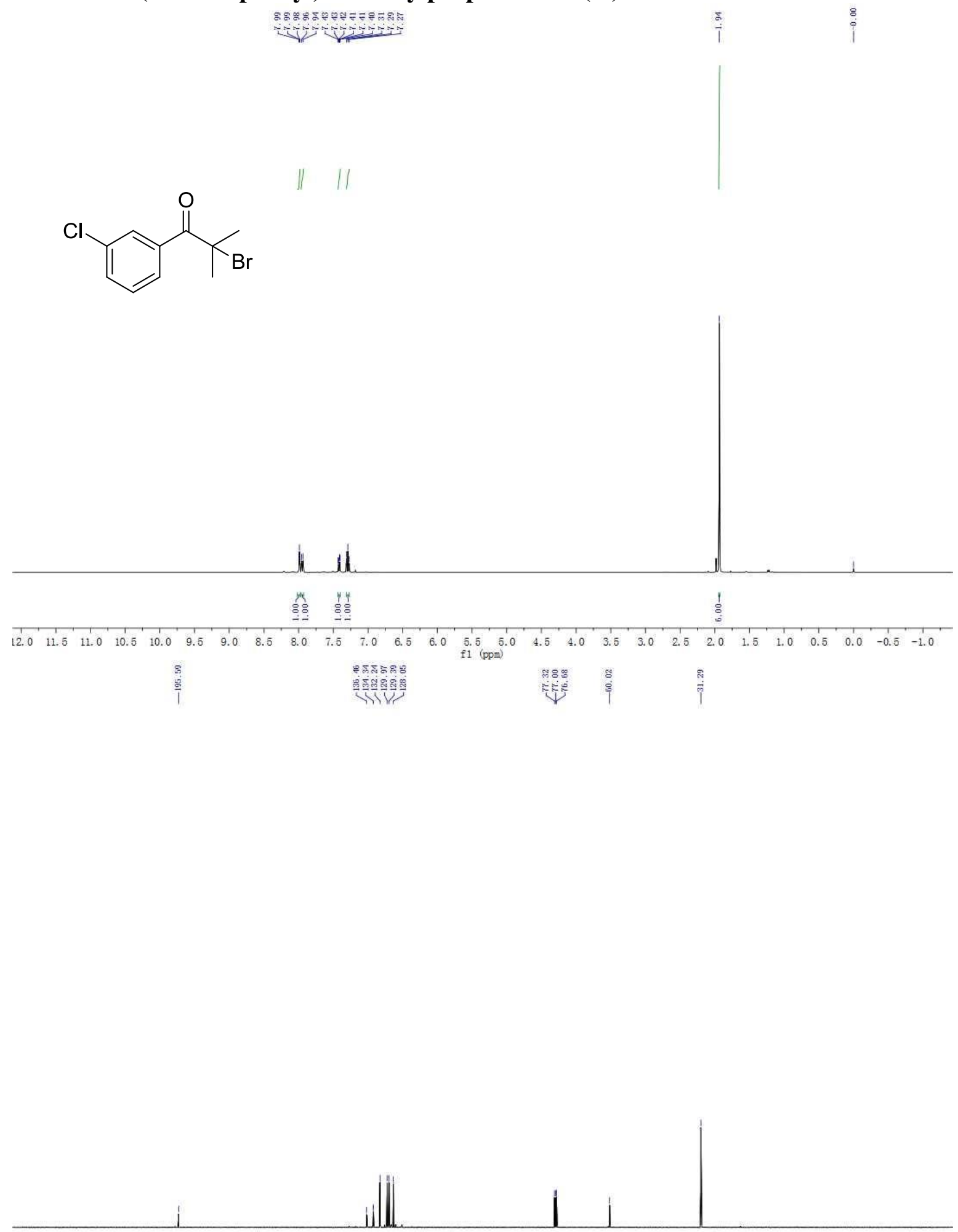

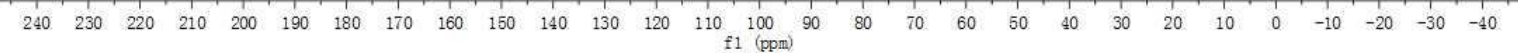


Ethyl 4-(2-bromo-2-methylpropanoyl)benzoate (1h)

ggag

एक

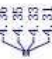

iे<smiles>CCOC(=O)c1ccc(C(=O)C(C)(C)Br)cc1</smiles>

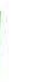
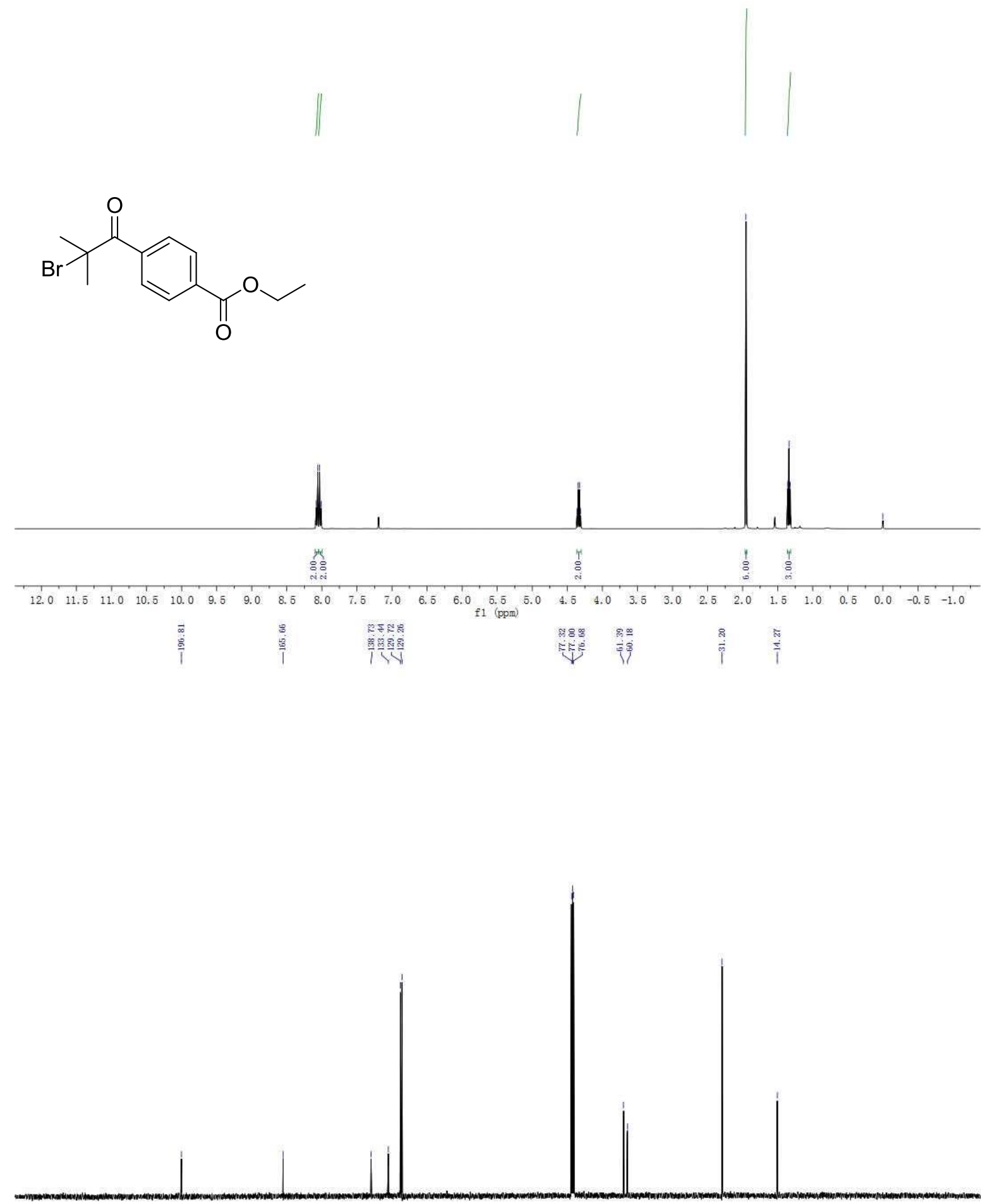

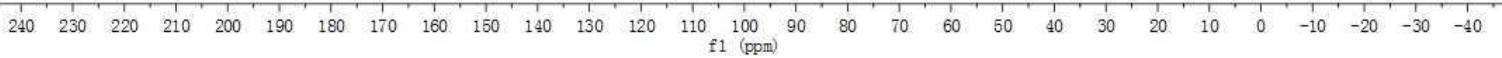


1-(4-Amino-3,5-dibromophenyl)-2-bromo-2-methylpropan-1-one (1i)

$i$<smiles>CC(C)(Br)C(=O)c1cc(Br)c(N)c(Br)c1</smiles>
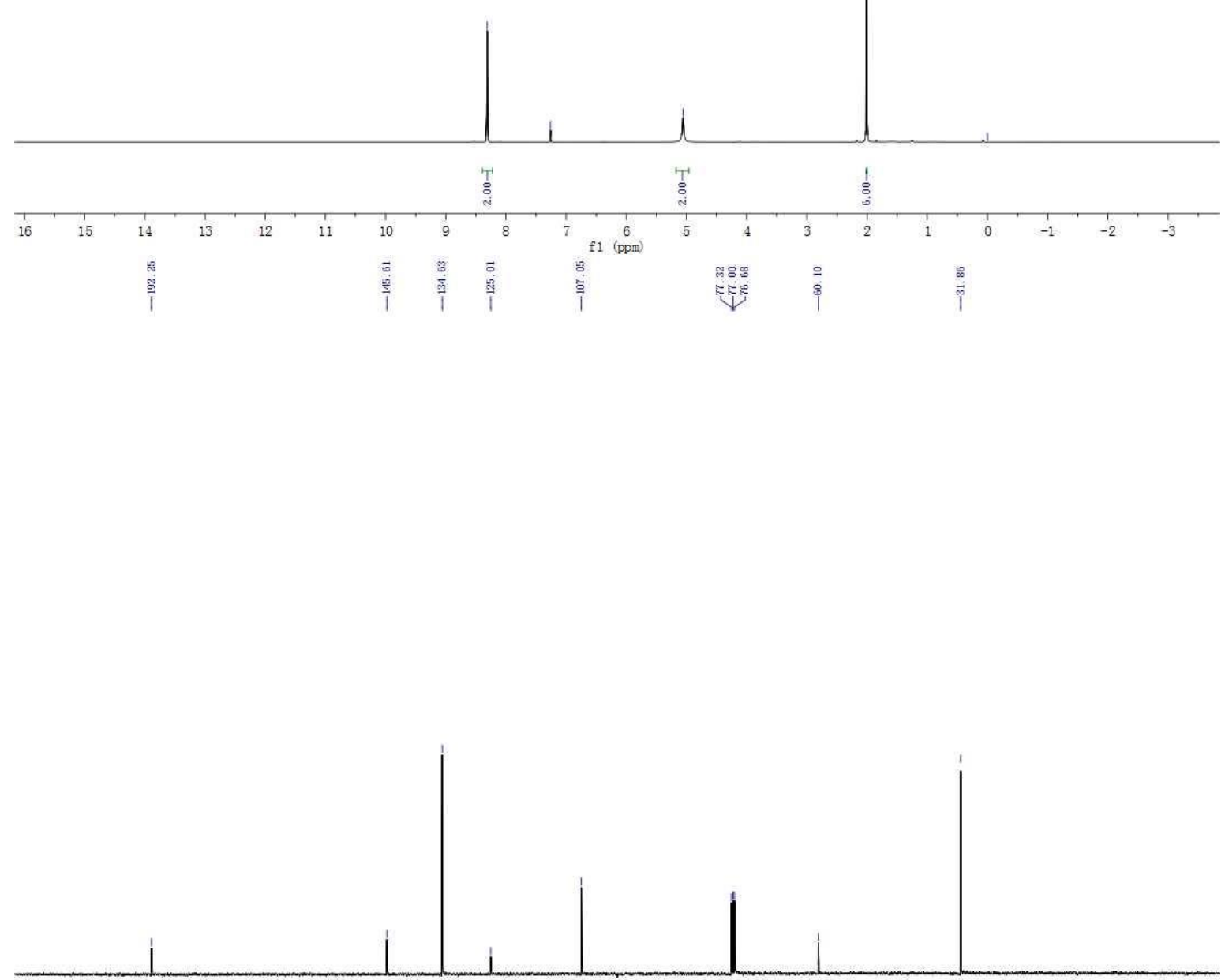
2-Bromo-2-methyl-1-(naphthalen-2-yl)propan-1-one (1j)

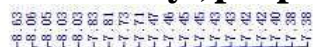
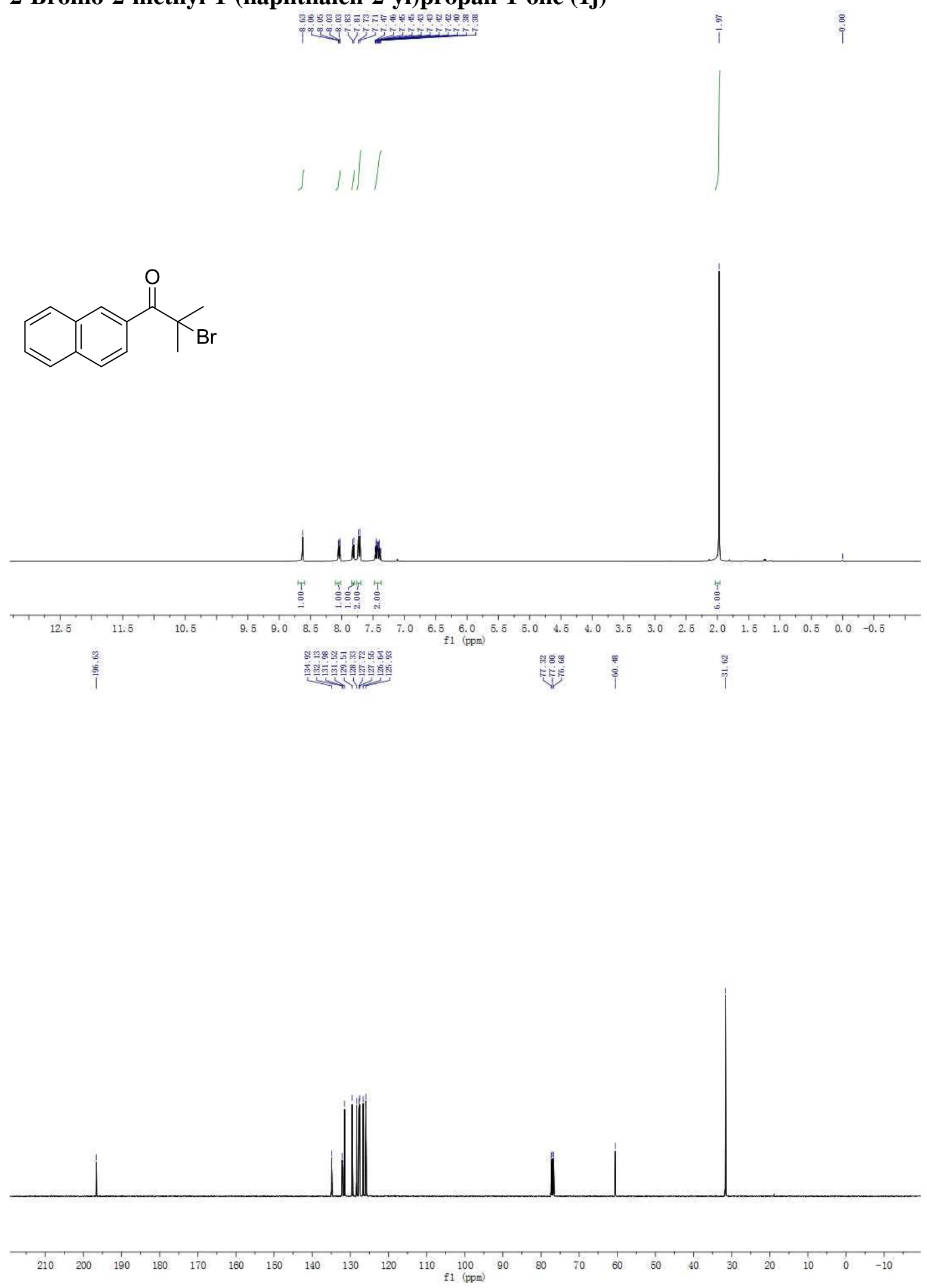

S50 
2-Bromo-2-methyl-1-(4-vinylphenyl)propan-1-one (1m)

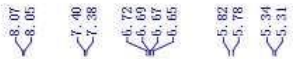

$\iint 1 / 1$

$C_{B r}$

)
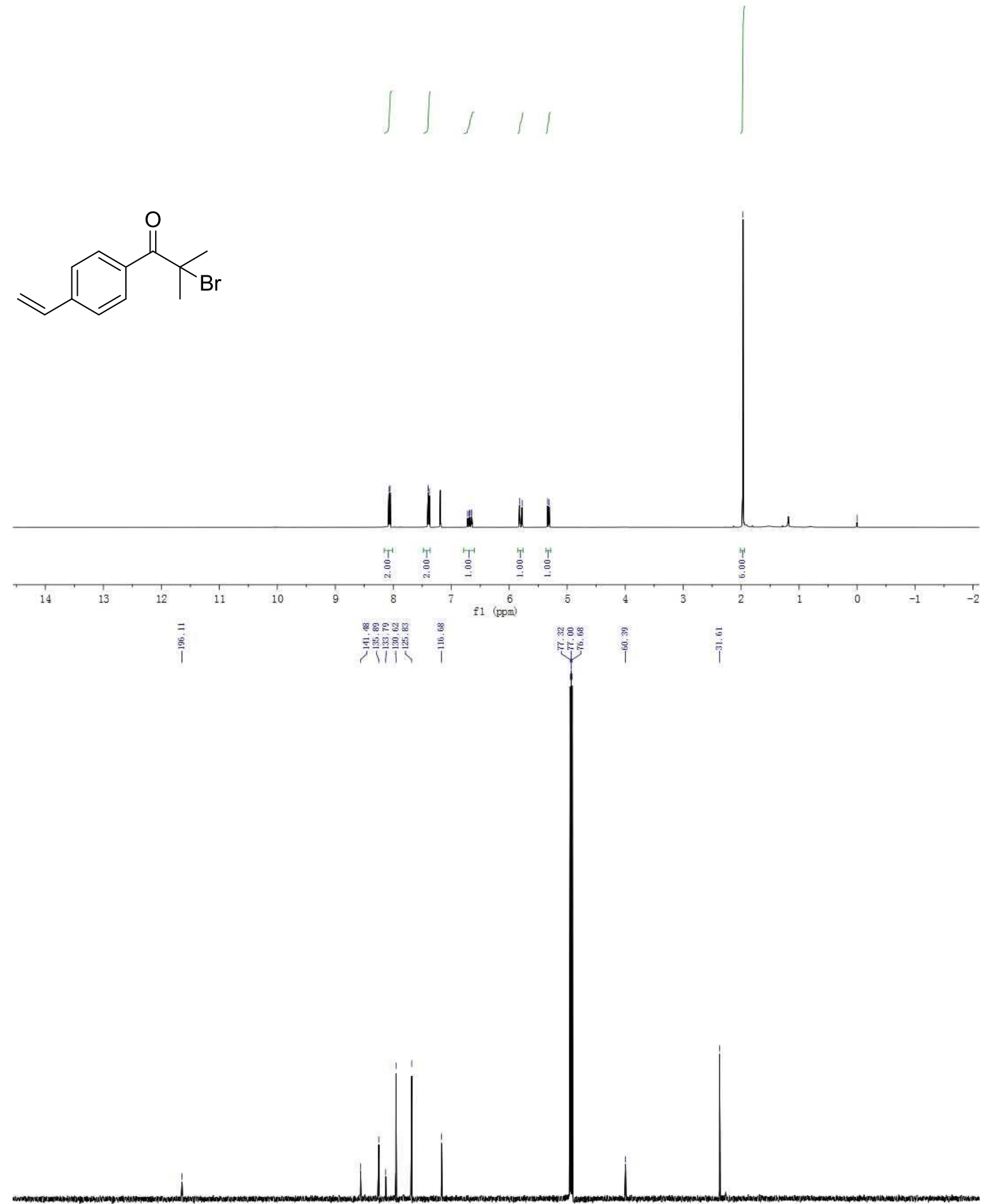

$\begin{array}{llllllllllllllllllllllllllllllllllllllllllllll}240 & 230 & 220 & 210 & 200 & 190 & 180 & 170 & 160 & 150 & 140 & 130 & 120 & 110 & 100 & 90 & 80 & 70 & 60 & 50 & 40 & 30 & 20 & 10 & 0 & -10 & -20 & -30 & -40\end{array}$ 
2-Bromo-2-methyl-1-(thiophen-3-yl)propan-1-one (1p)

कल
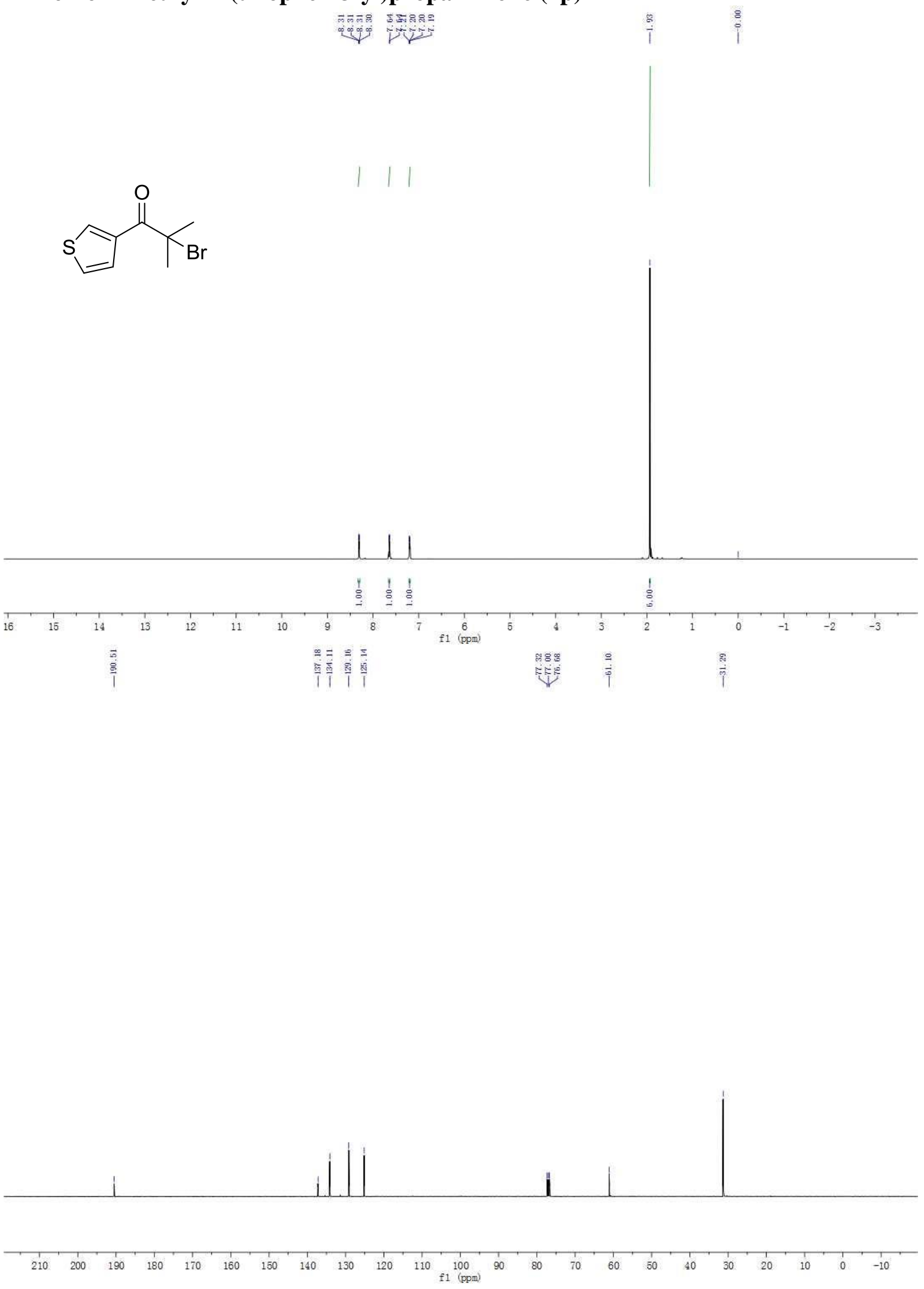

S52 
4-Acetylphenyl 2-bromo-2-methylpropanoate (1r)

要
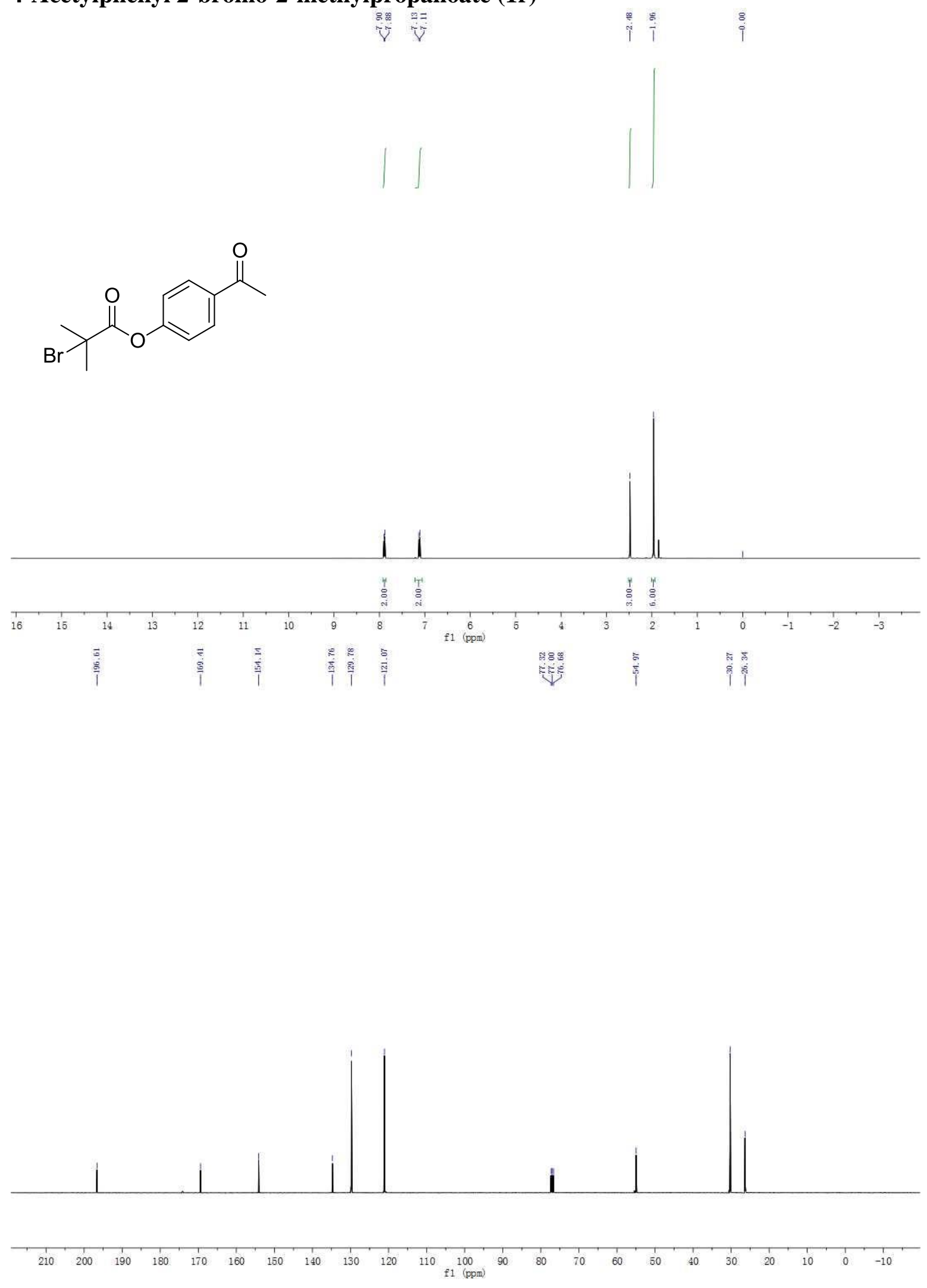

S53 
2-bromo-3-methoxy-2-methyl-1-phenylpropan-1-one (1x) ${ }^{\left[\frac{89]}{}\right.}$

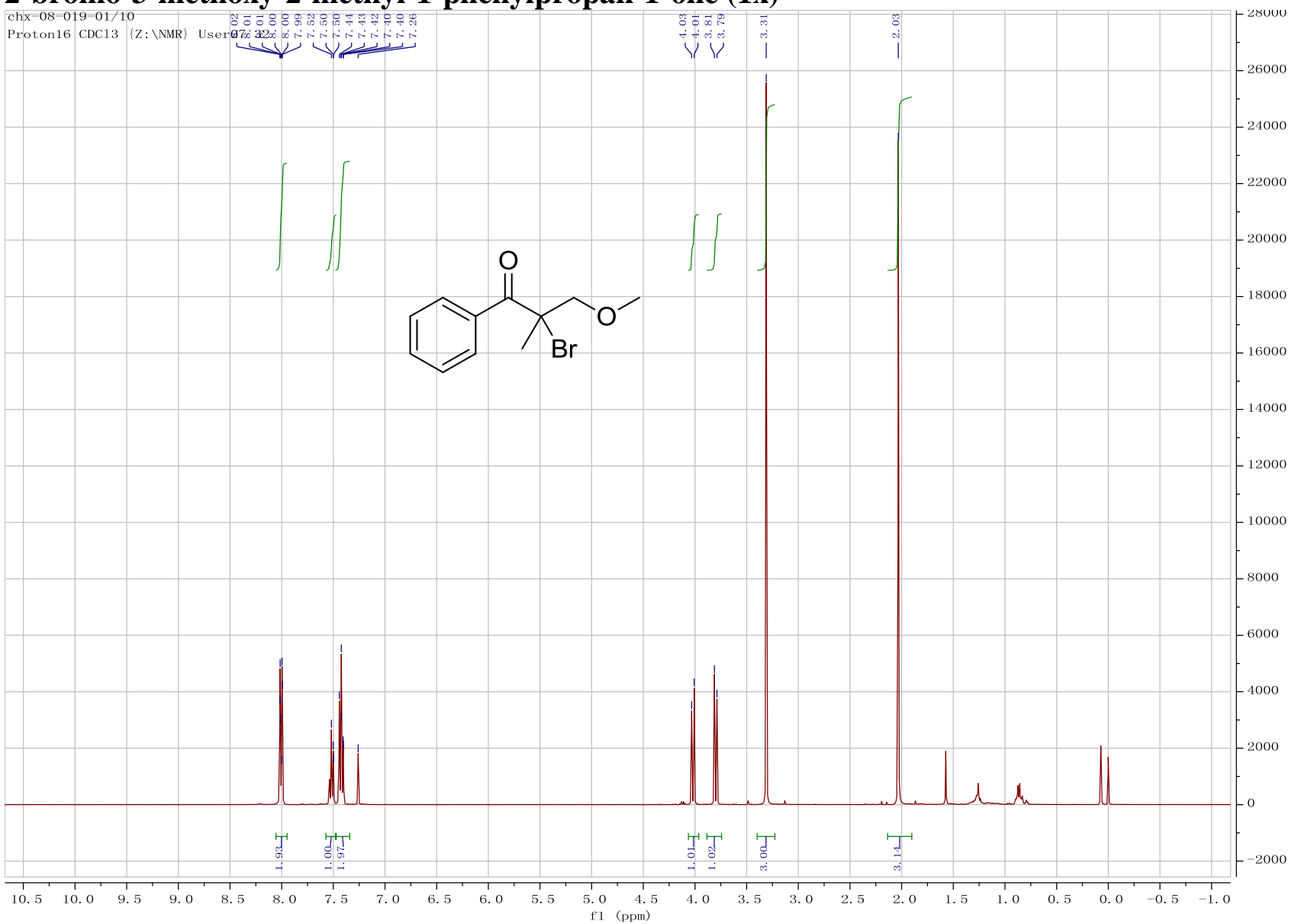


2-bromo-2-cyclopropyl-1-phenylethanone (1y)

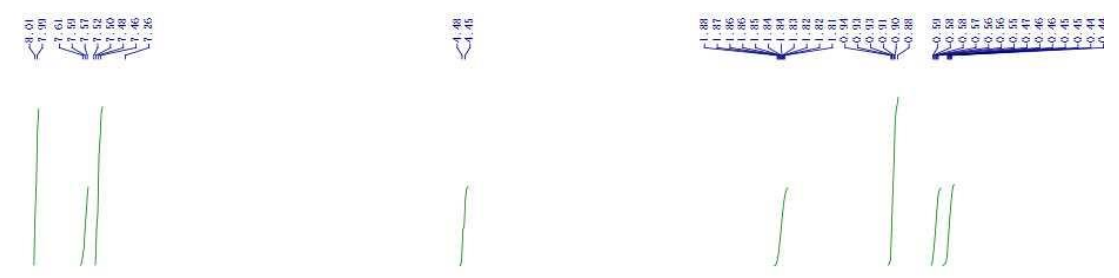<smiles>O=C(c1ccccc1)C(Br)C1CC1</smiles>

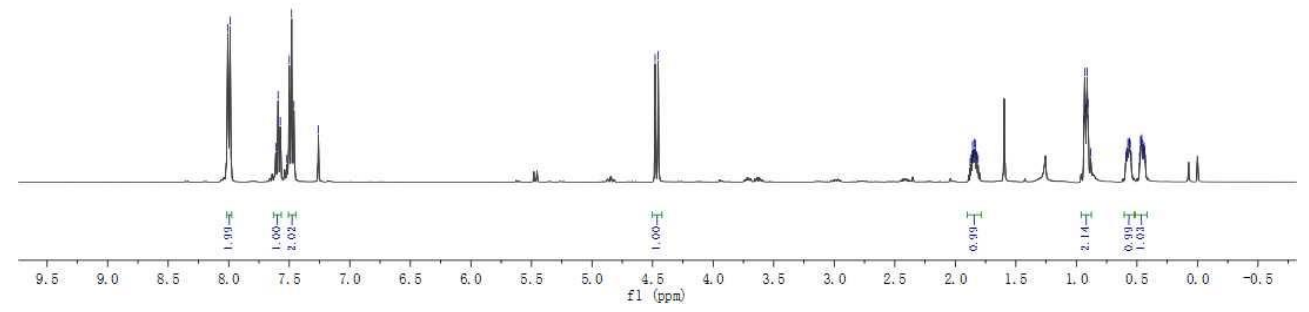


Di-tert-butyl-4-(4-methoxybenzyl)-2,6-dimethyl-1,4-dihydropyridine-3,5-dicarboxylate (2d)

$$
\text { sू. }
$$

1
0<smiles>CCCCOC(=O)C1=C(C)NC(C)=C(C(=O)OCC)C1Cc1ccc(OC)cc1</smiles>

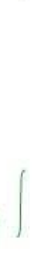

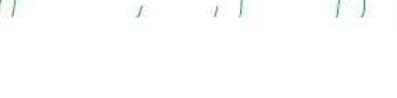
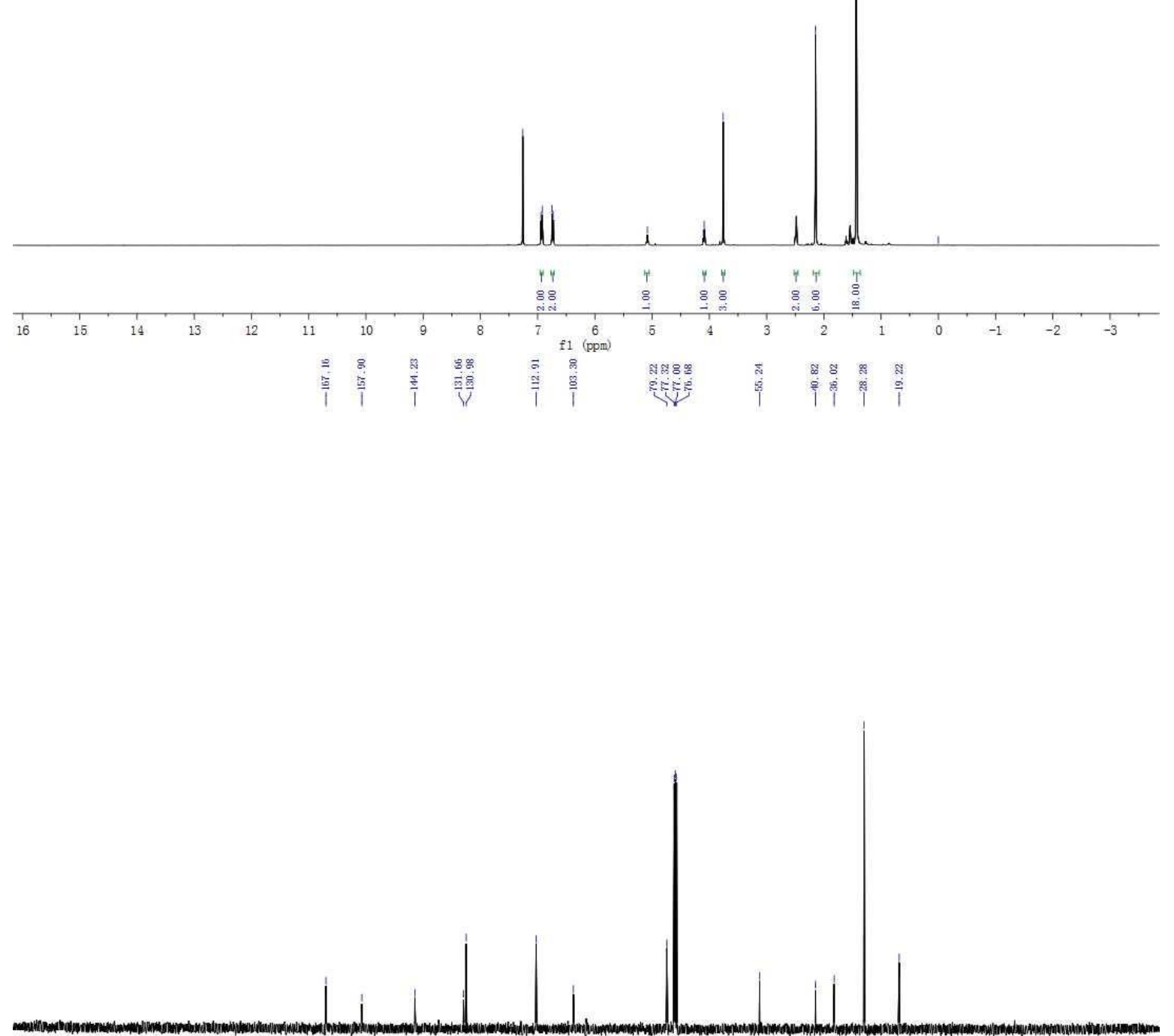

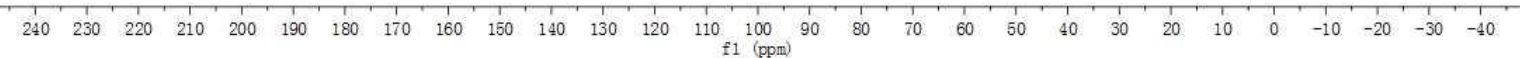


Di-tert-butyl-4-(4-chlorobenzyl)-2,6-dimethyl-1,4-dihydropyridine-3,5-dicarboxylate(2e)

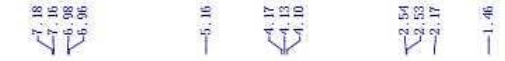

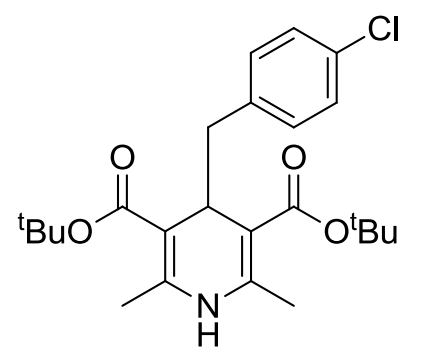<smiles>CCCC</smiles>
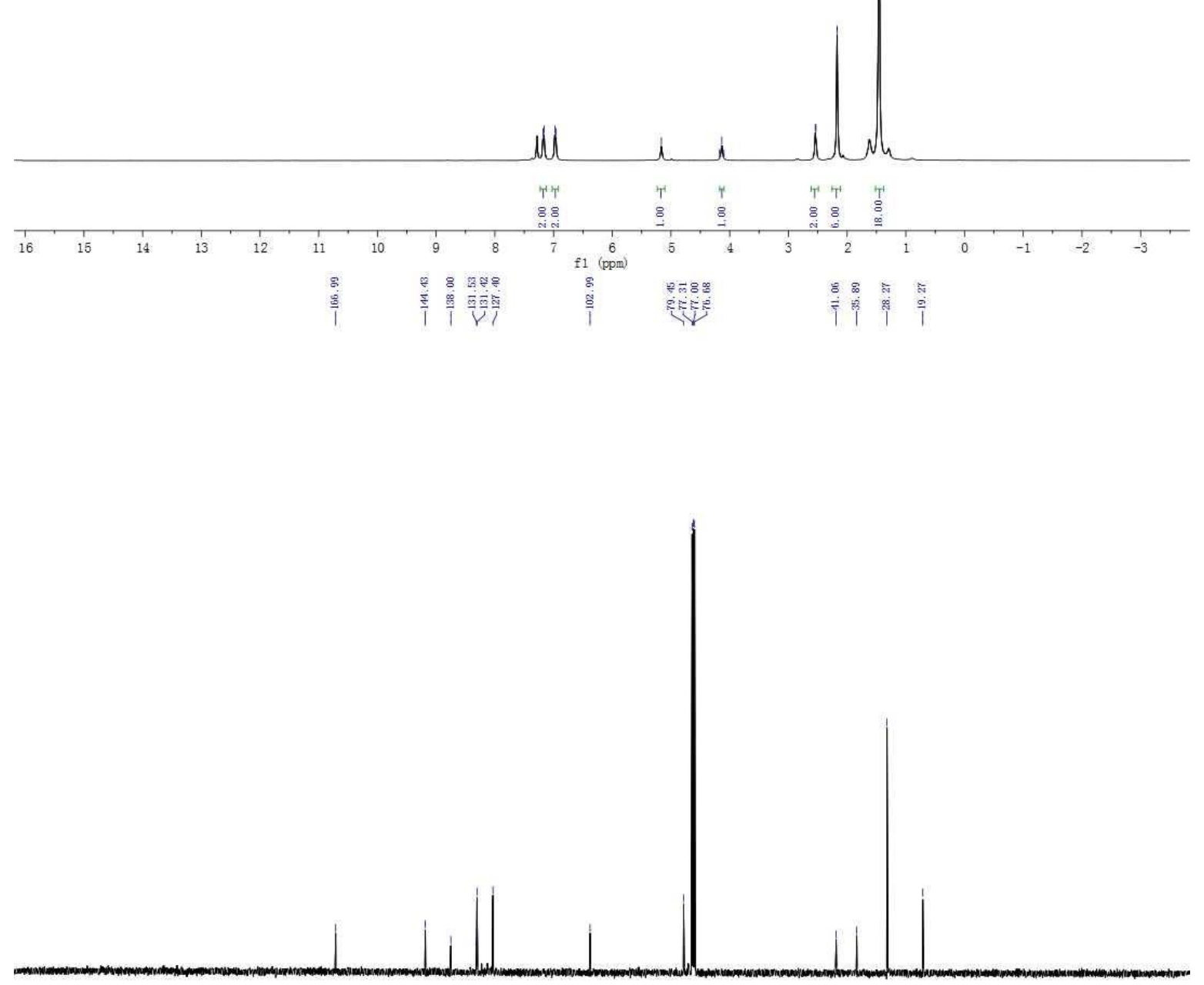

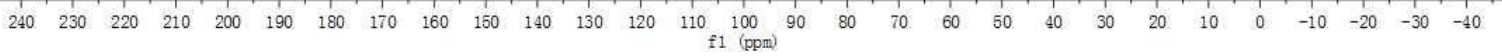


Di-tert-butyl-2,6-dimethyl-4-(thiophen-2-ylmethyl)-1,4-dihydropyridine-3,5-dicarboxylate(2f)<smiles>CCCCOC(=O)C1=C(C)NC(C)=C(C(=O)OCC)C1Cc1cccs1</smiles>
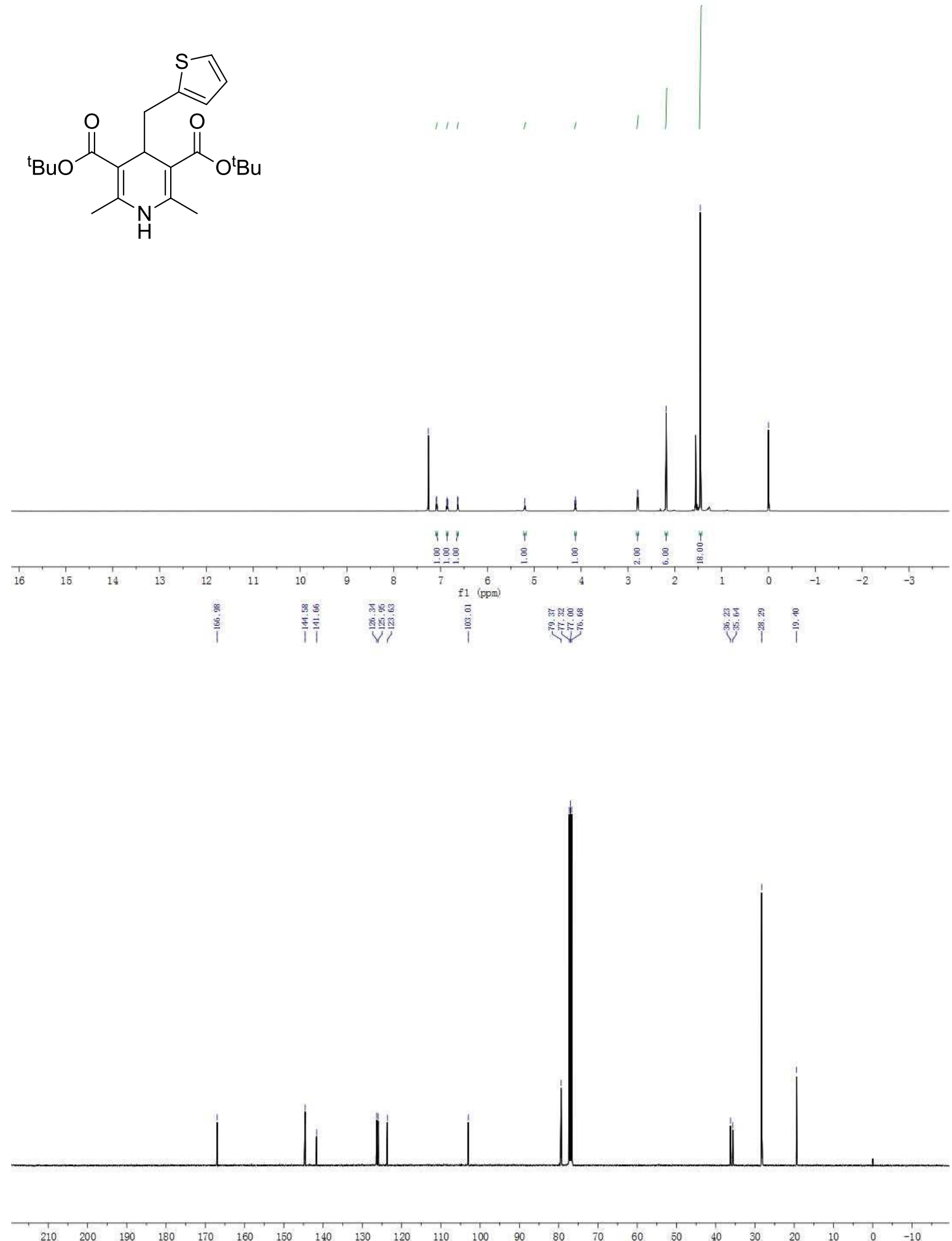

$140 \quad 130$ $110 \underset{f 1}{100}(\mathrm{ppm})$ 
Di-tert-butyl-2,6-dimethyl-4-(1-phenylethyl)-1,4-dihydropyridine-3,5-dicarboxylate(2g)

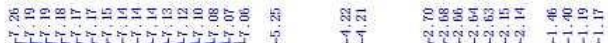<smiles>CCCCOC(=O)C1=C(C)NC(C)=C(C(=O)OCCC)C1C(C)c1ccccc1</smiles>

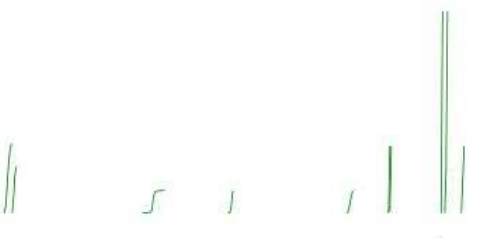<smiles>CCCCC1CCCCC1</smiles>
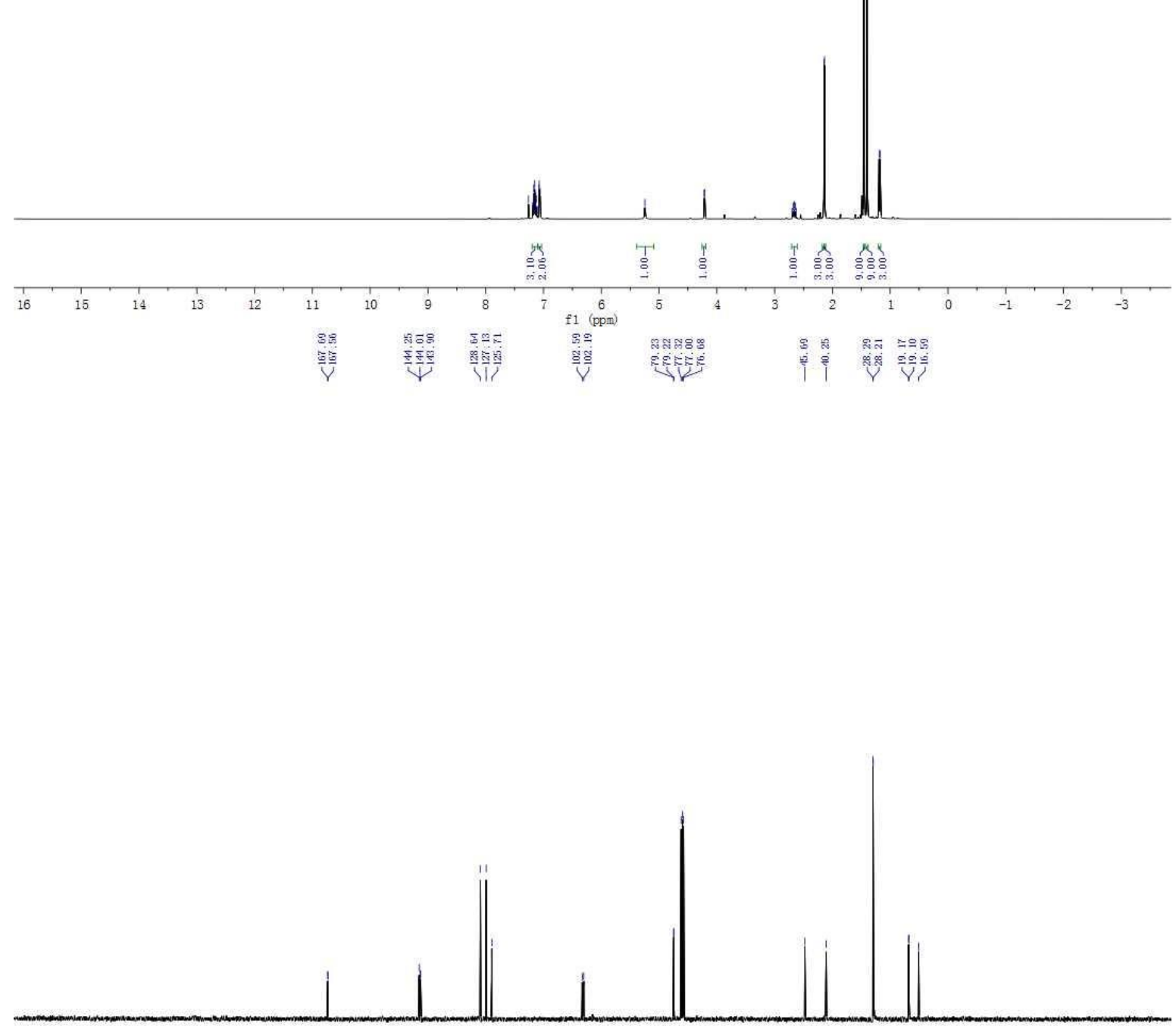

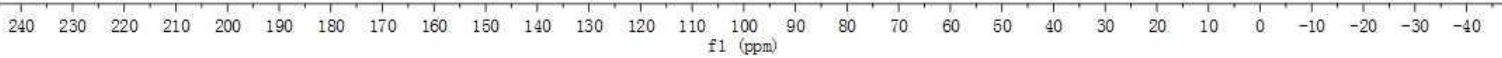


2,2-Dimethyl-1,3-diphenylpropan-1-one (3a)

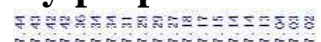
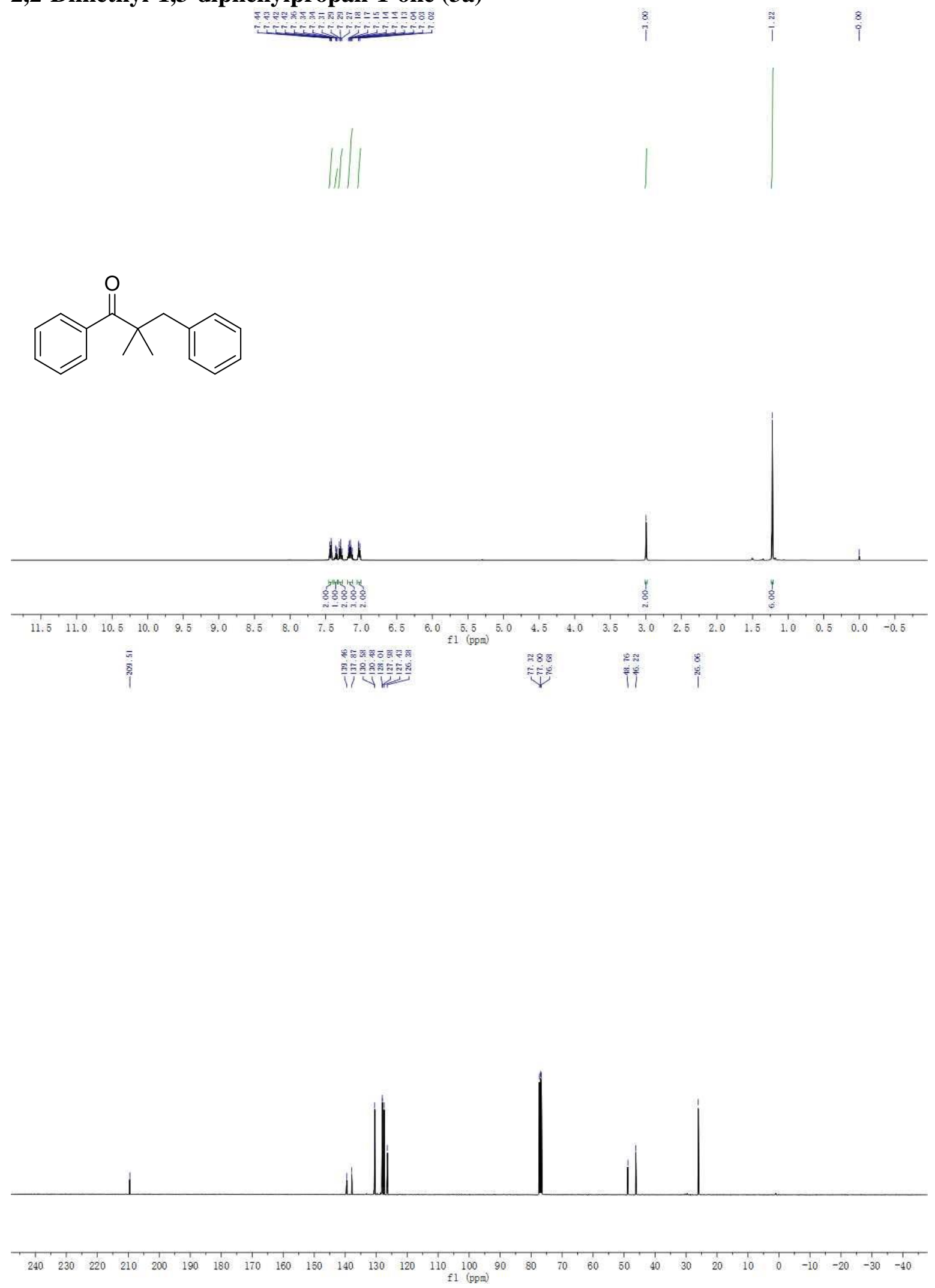

S60 
2,2-Dimethyl-3-phenyl-1-(p-tolyl)propan-1-one (3b)
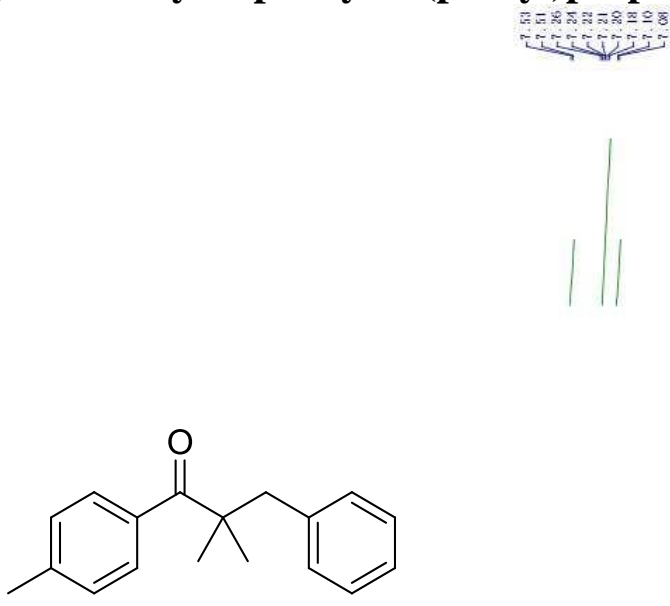

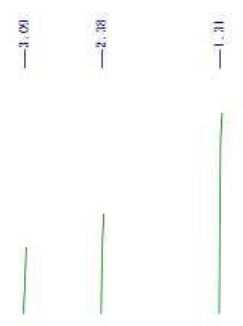

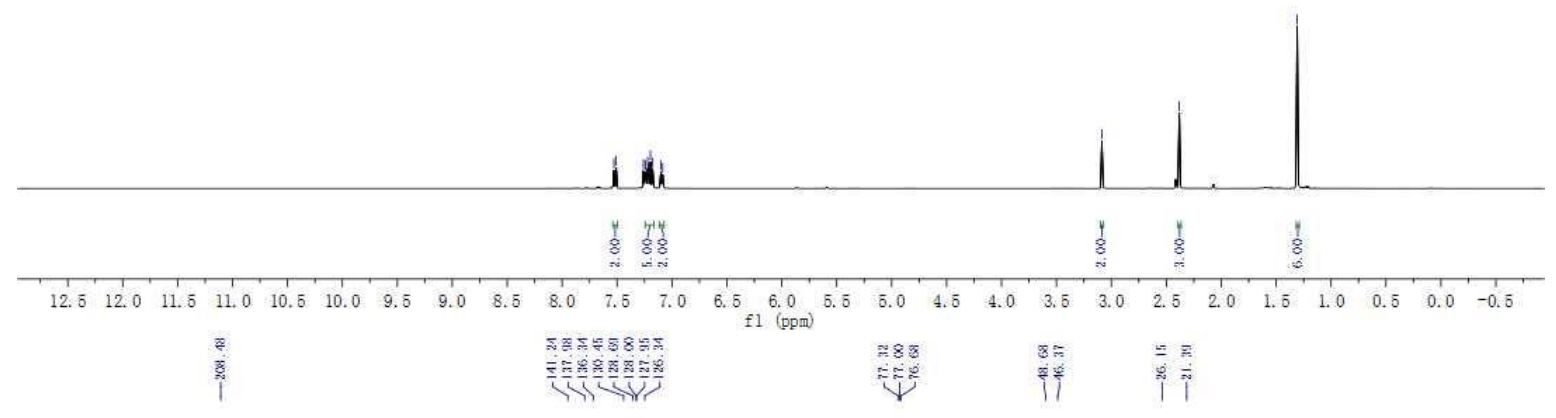
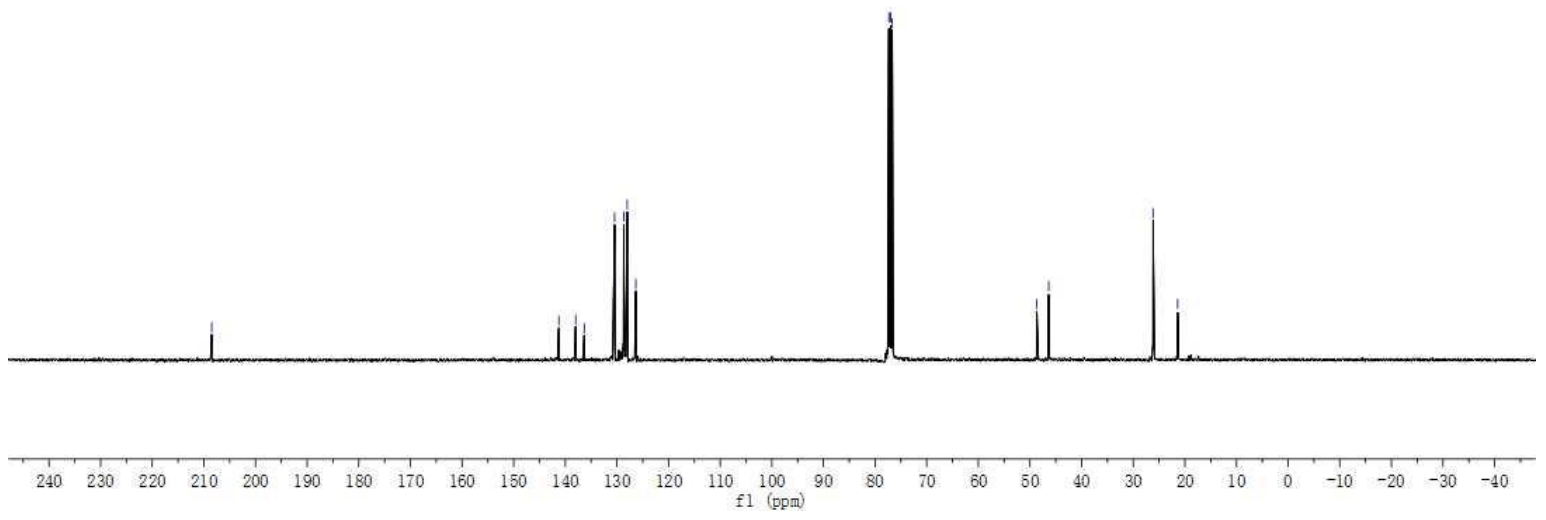

S61 
1-(4-Methoxyphenyl)-2,2-dimethyl-3-phenylpropan-1-one (3c)
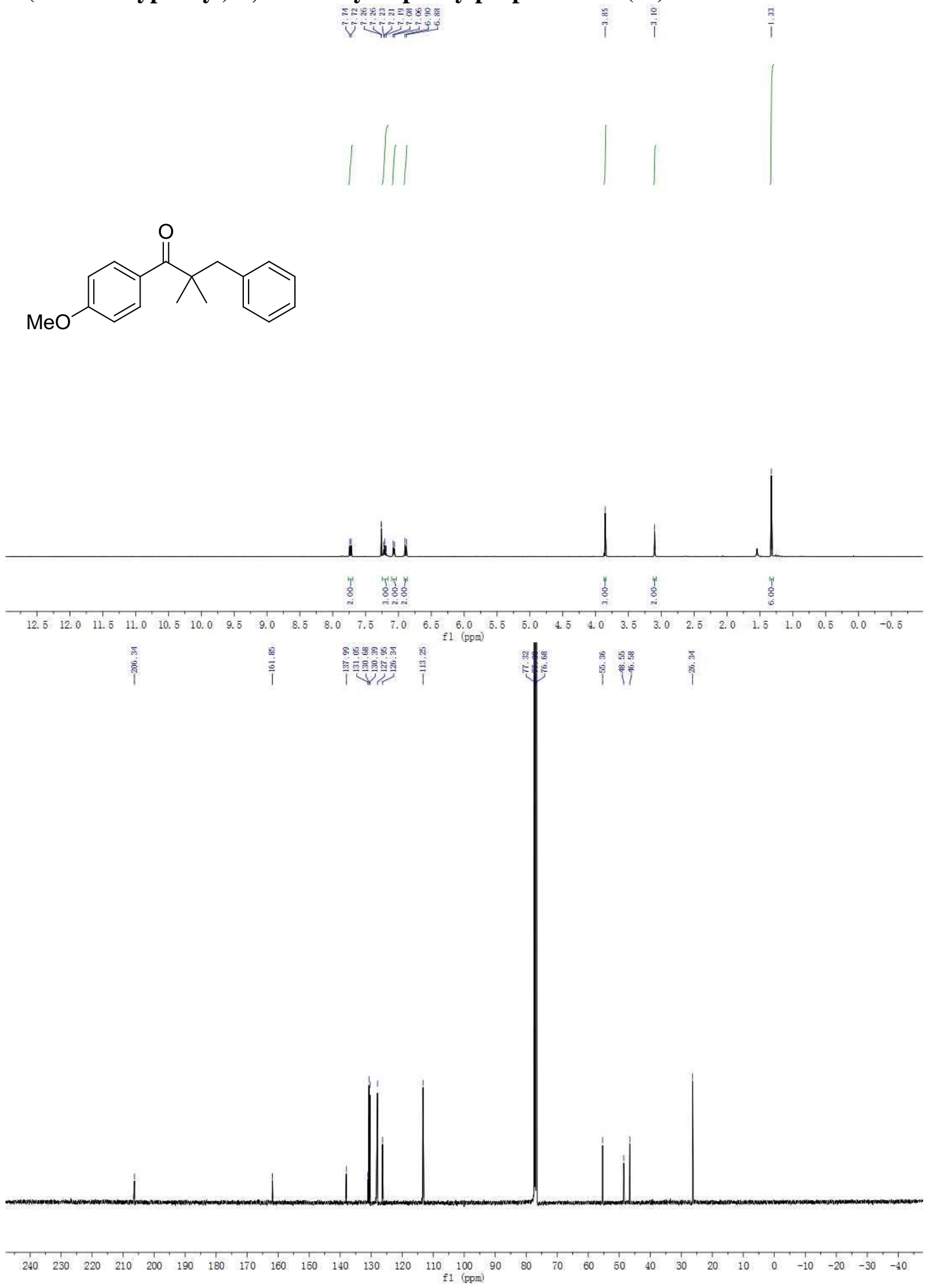

S62 
1-(4-Fluorophenyl)-2,2-dimethyl-3-phenylpropan-1-one (3d)

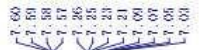

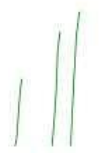
$\stackrel{5}{i}$
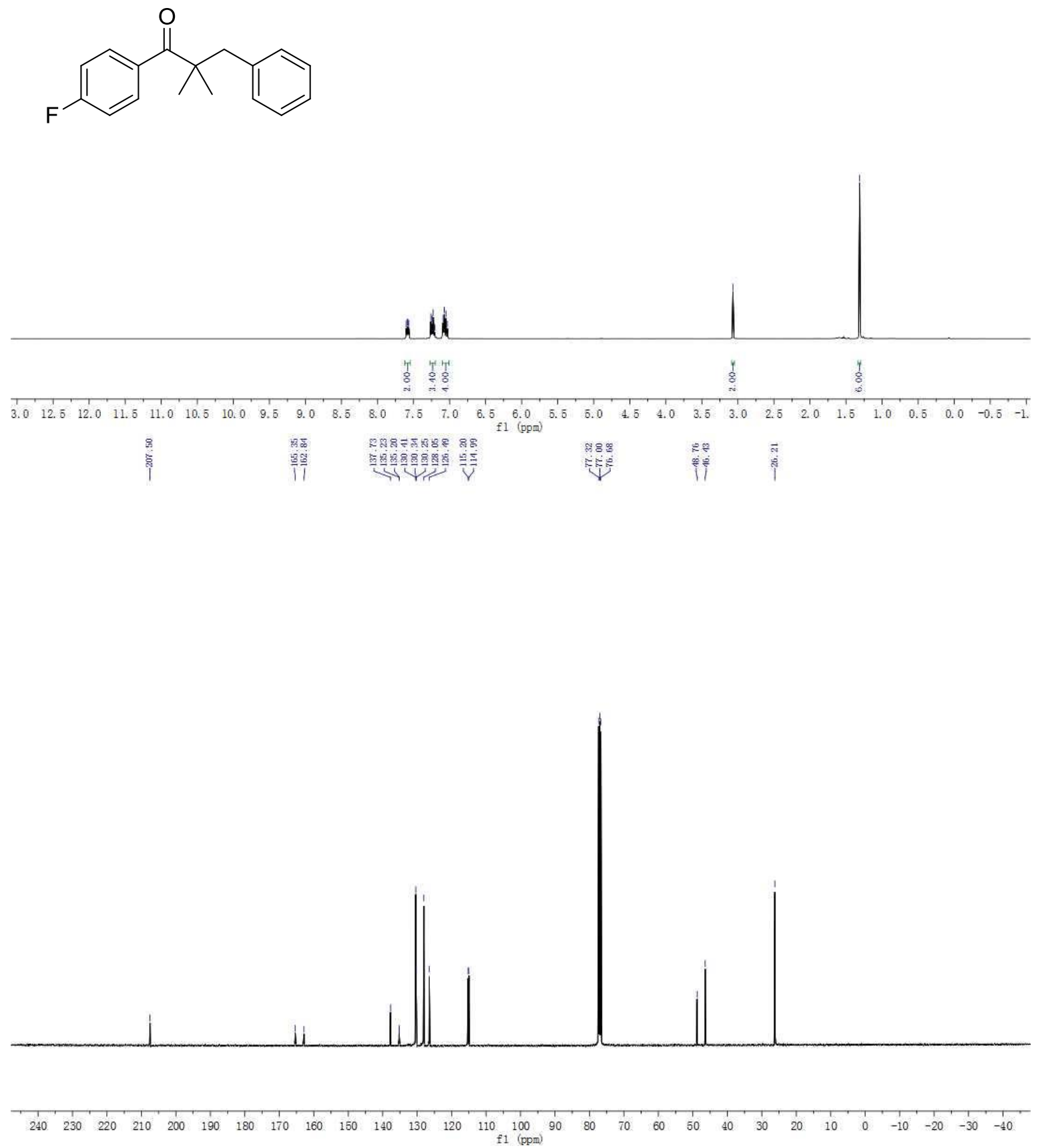

S63 
1-(3-Fluorophenyl)-2,2-dimethyl-3-phenylpropan-1-one (3e)

견대
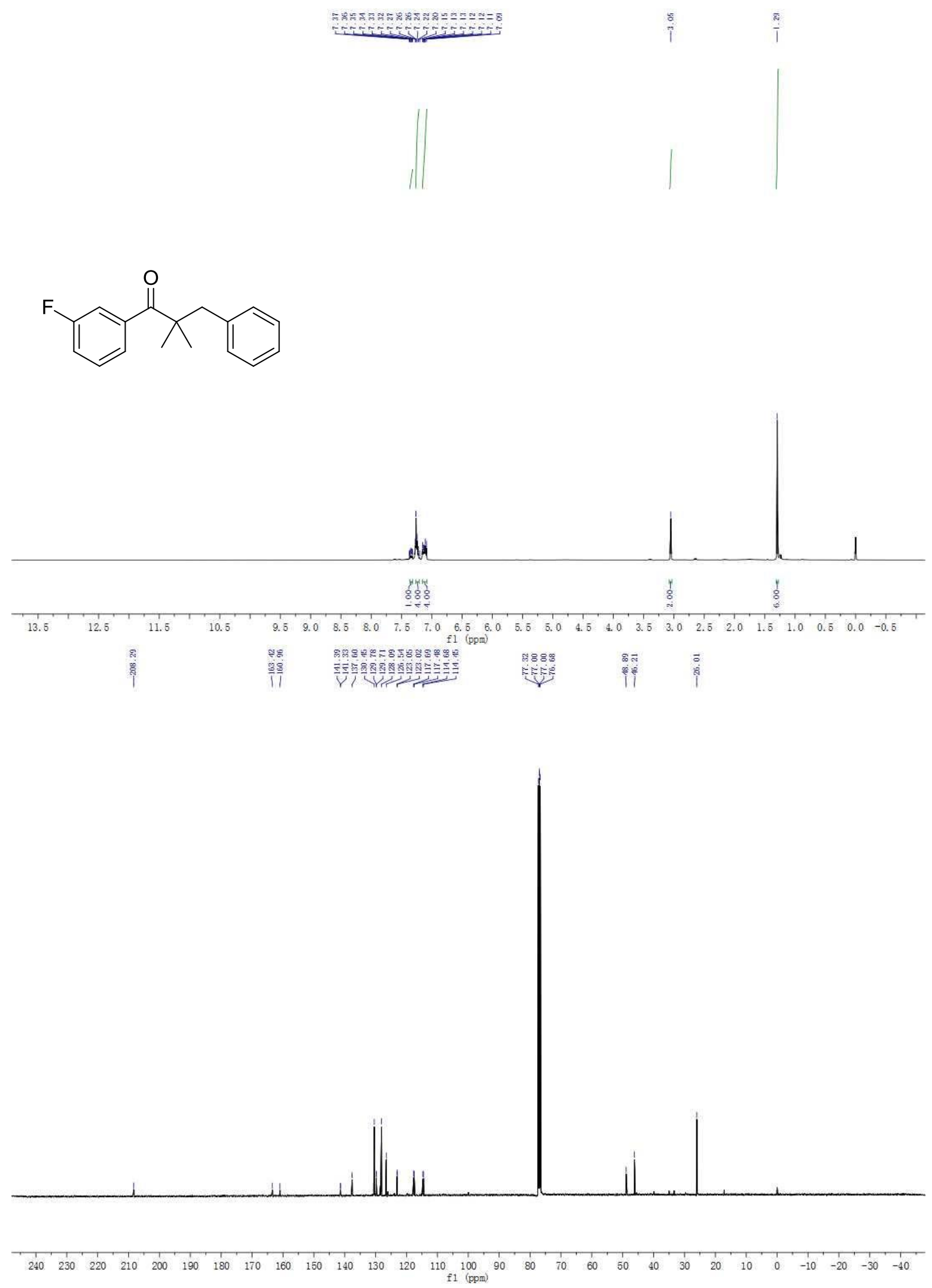

S64 
1-(3-Chlorophenyl)-2,2-dimethyl-3-phenylpropan-1-one (3f)
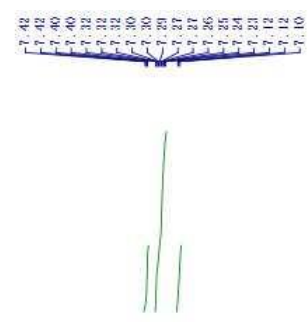

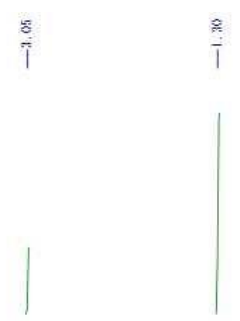

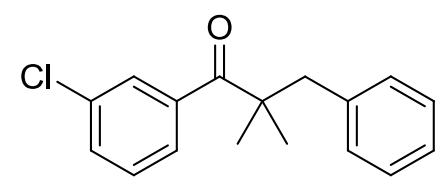
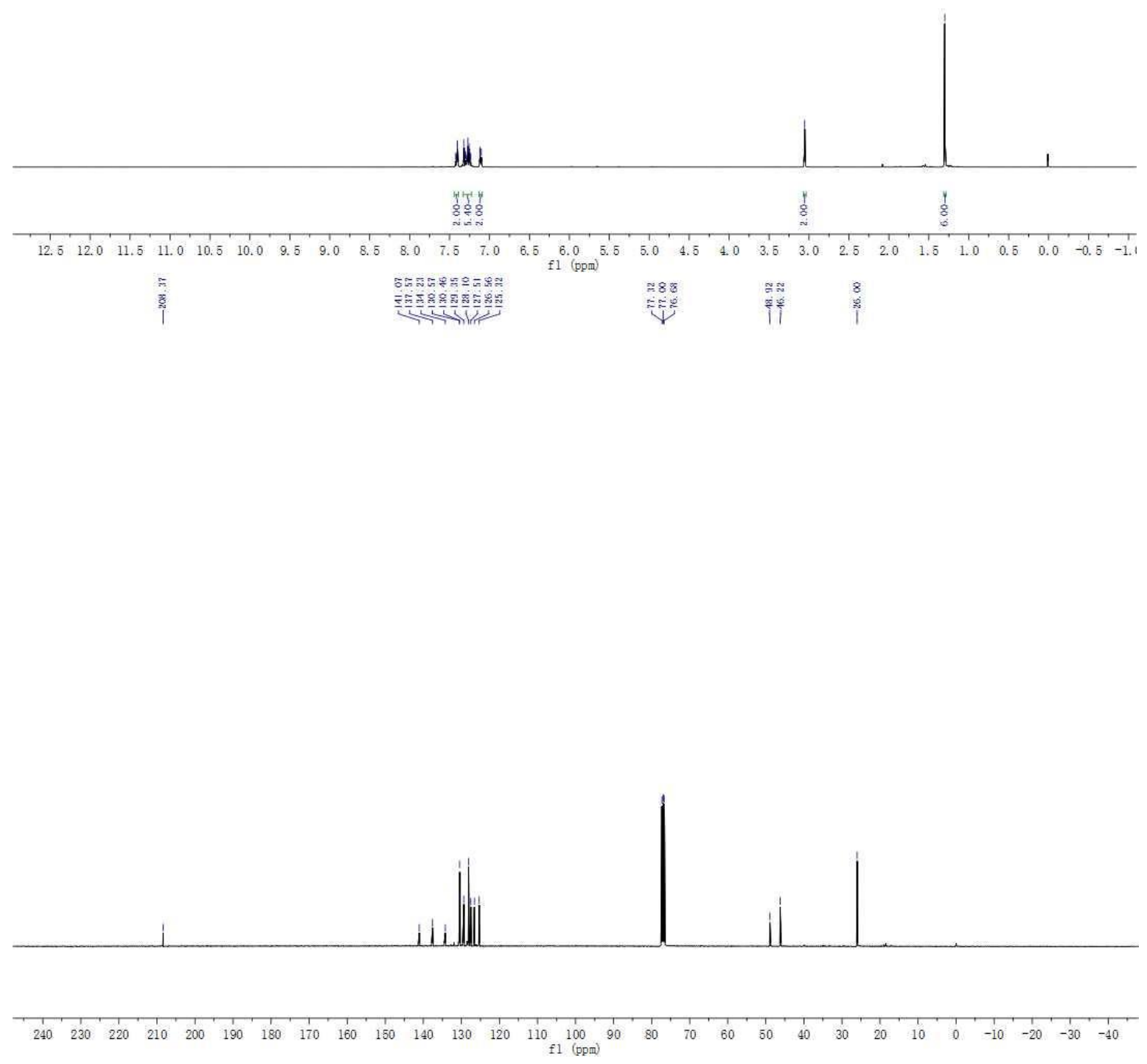

S65 
1-Mesityl-2,2-dimethyl-3-phenylpropan-1-one (3g)

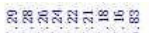

itisition
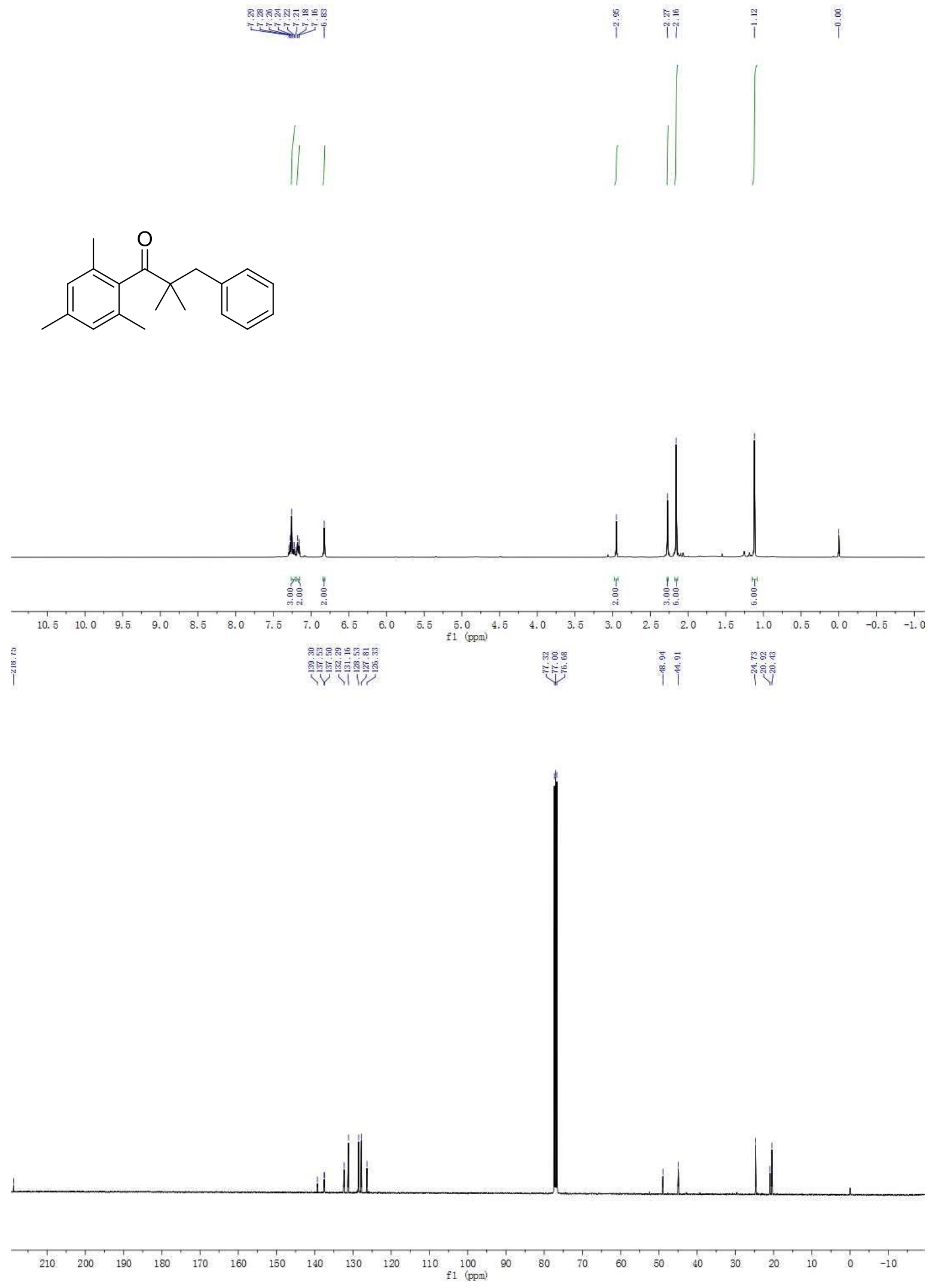

S66 
Ethyl 4-(2,2-dimethyl-3-phenylpropanoyl)benzoate (3h)
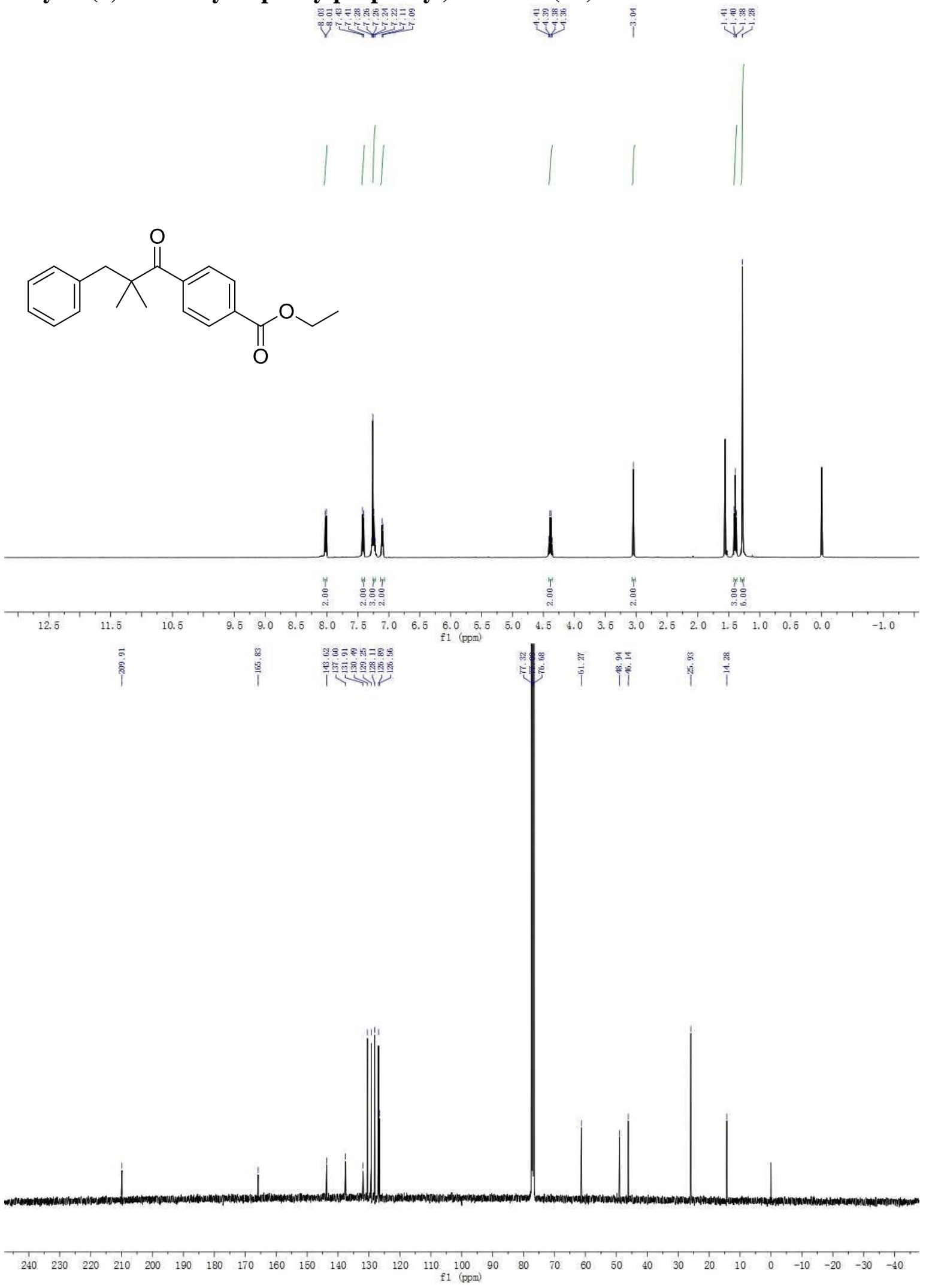

S67 
1-(4-Amino-3,5-dibromophenyl)-2,2-dimethyl-3-phenylpropan-1-one (3i)
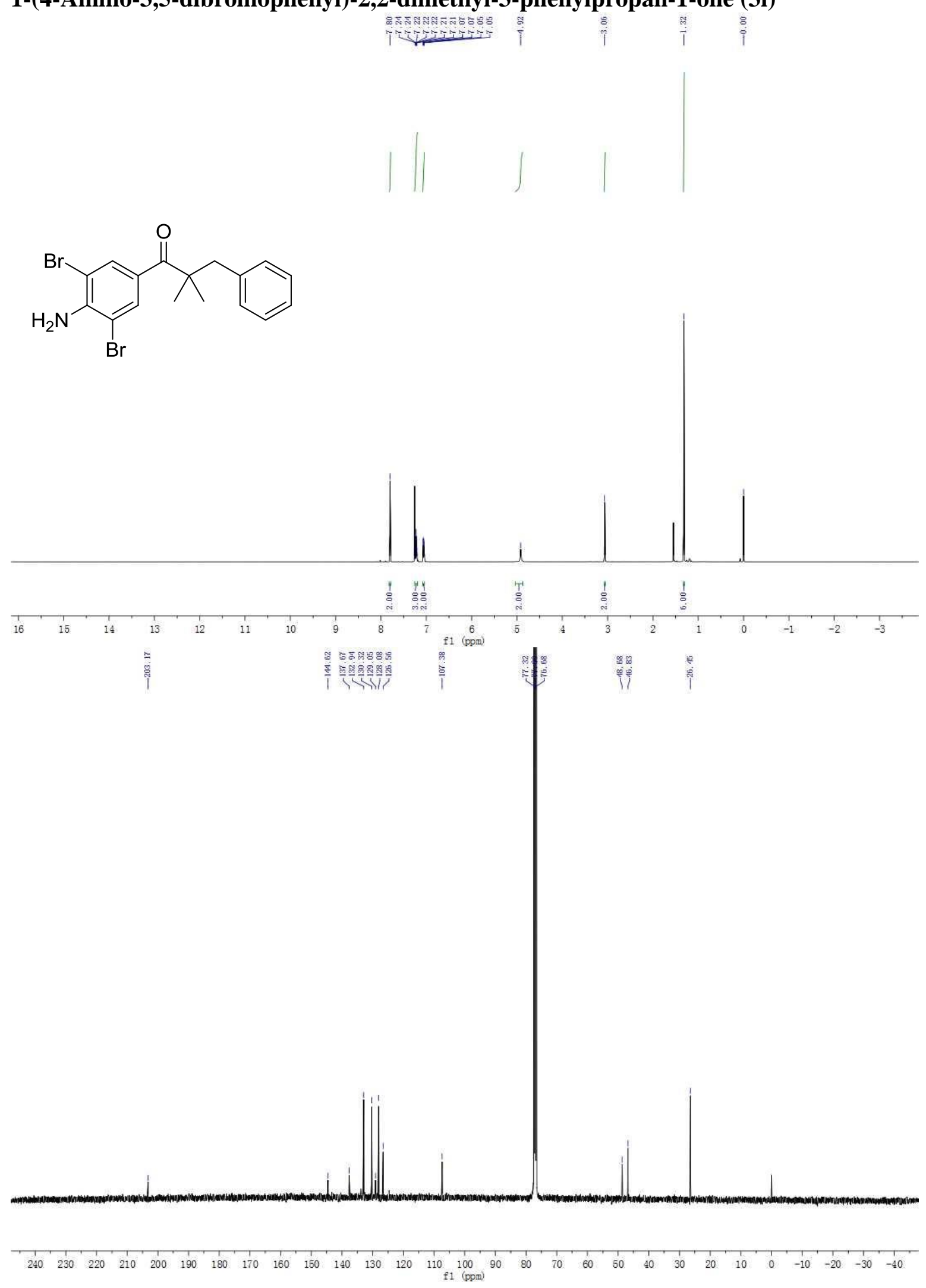

S68 
2,2-Dimethyl-1-(naphthalen-2-yl)-3-phenylpropan-1-one (3i)

ํำ ๒

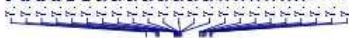
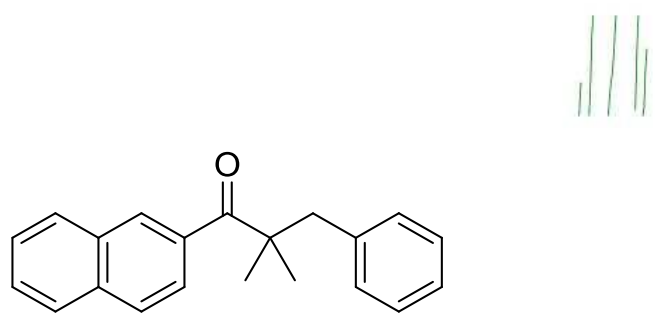

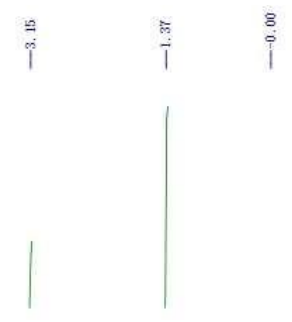

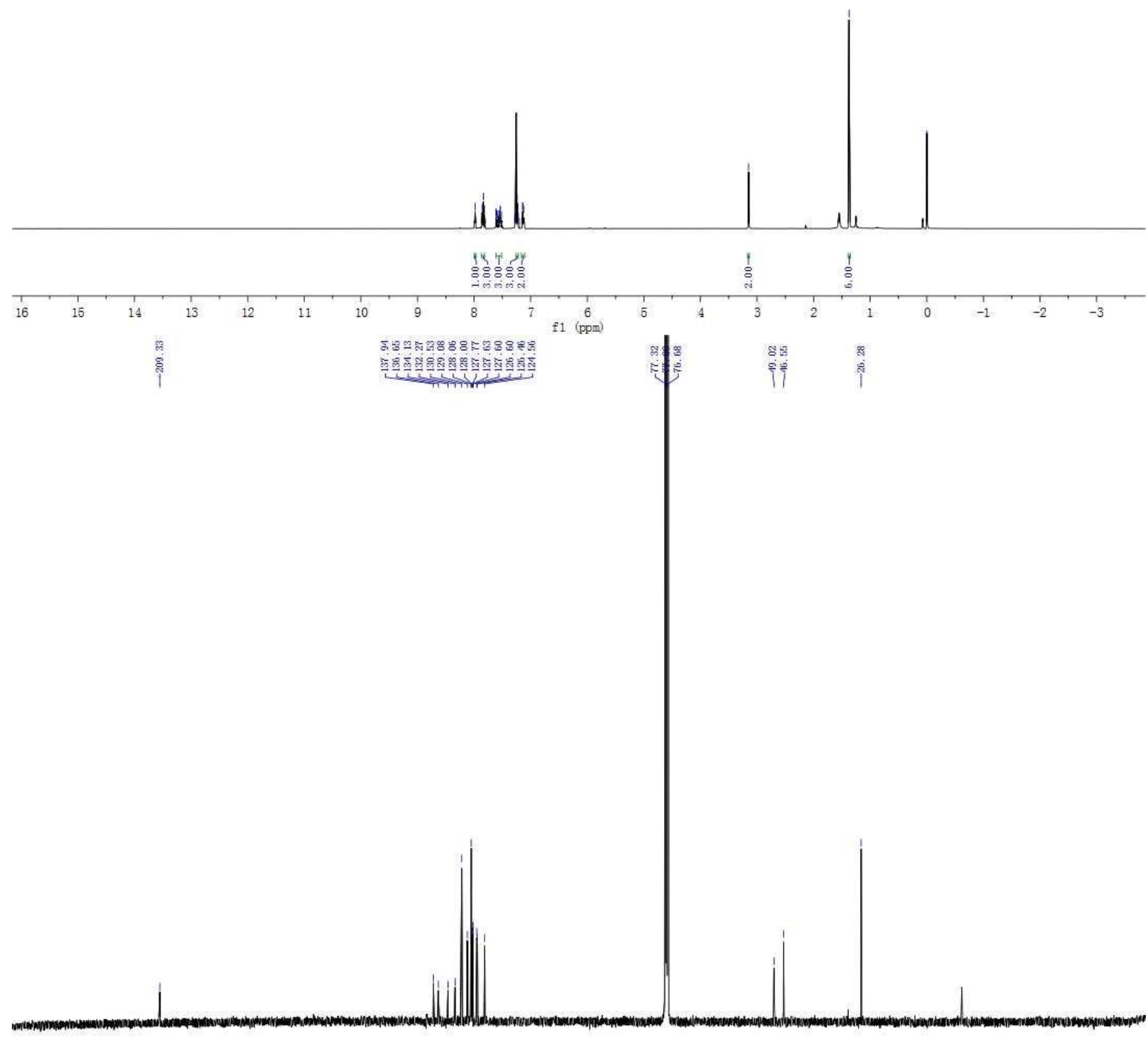

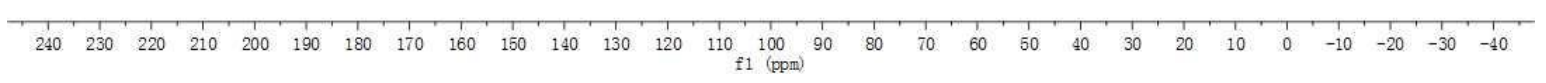

S69 


\section{(1-Benzylcyclopentyl)(phenyl)methanone (3k)}

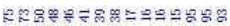

istivitivition

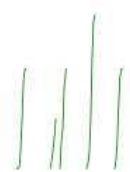

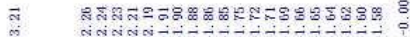

i<smiles>O=C(c1ccccc1)C1(Cc2ccccc2)CCCC1</smiles>

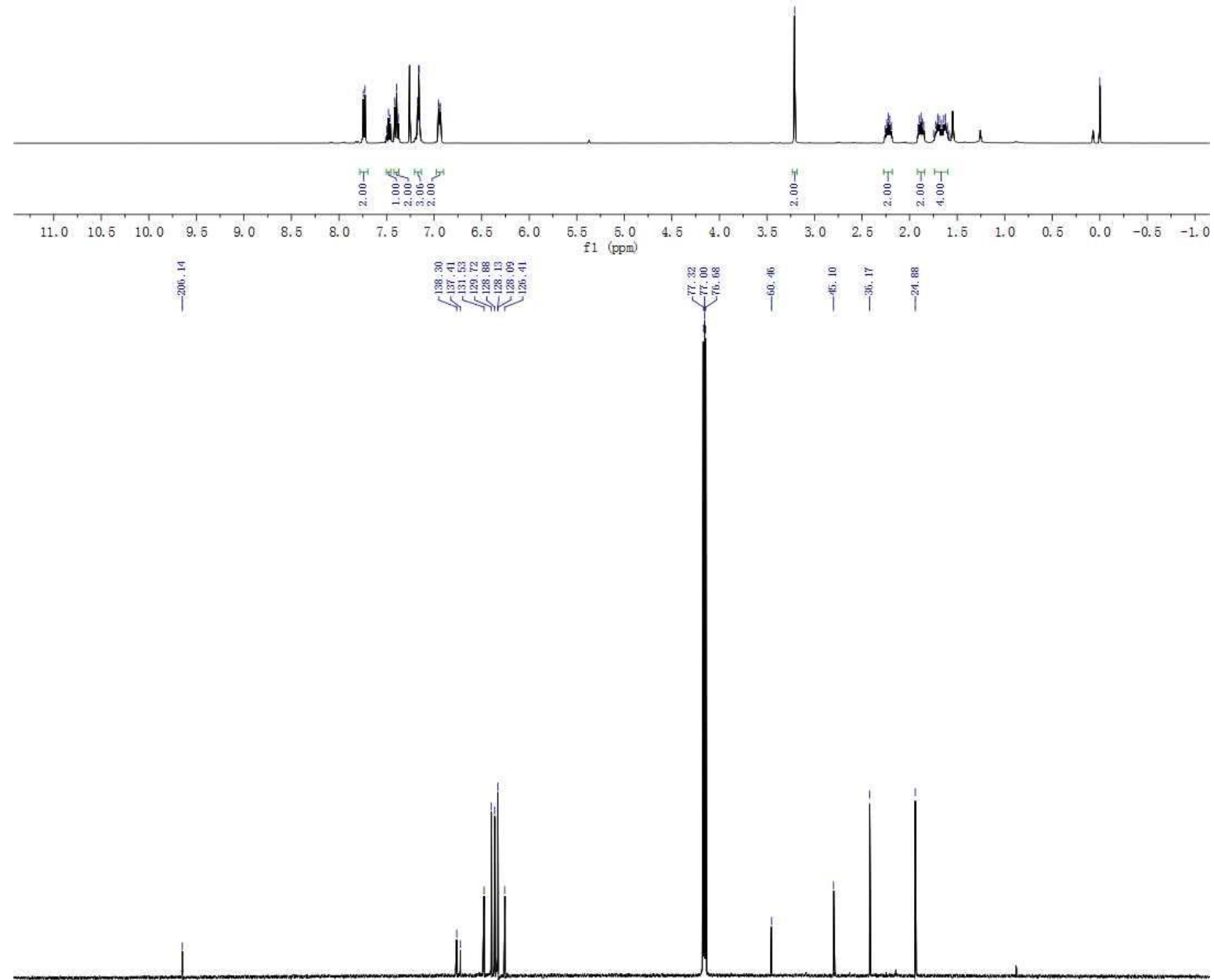

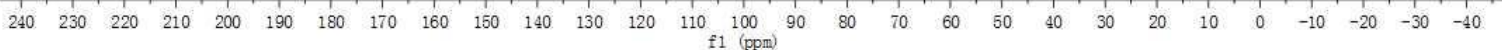


(1-Benzylcyclohexyl)(phenyl)methanone (3I)

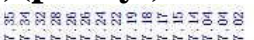

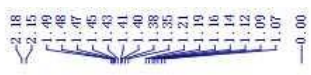
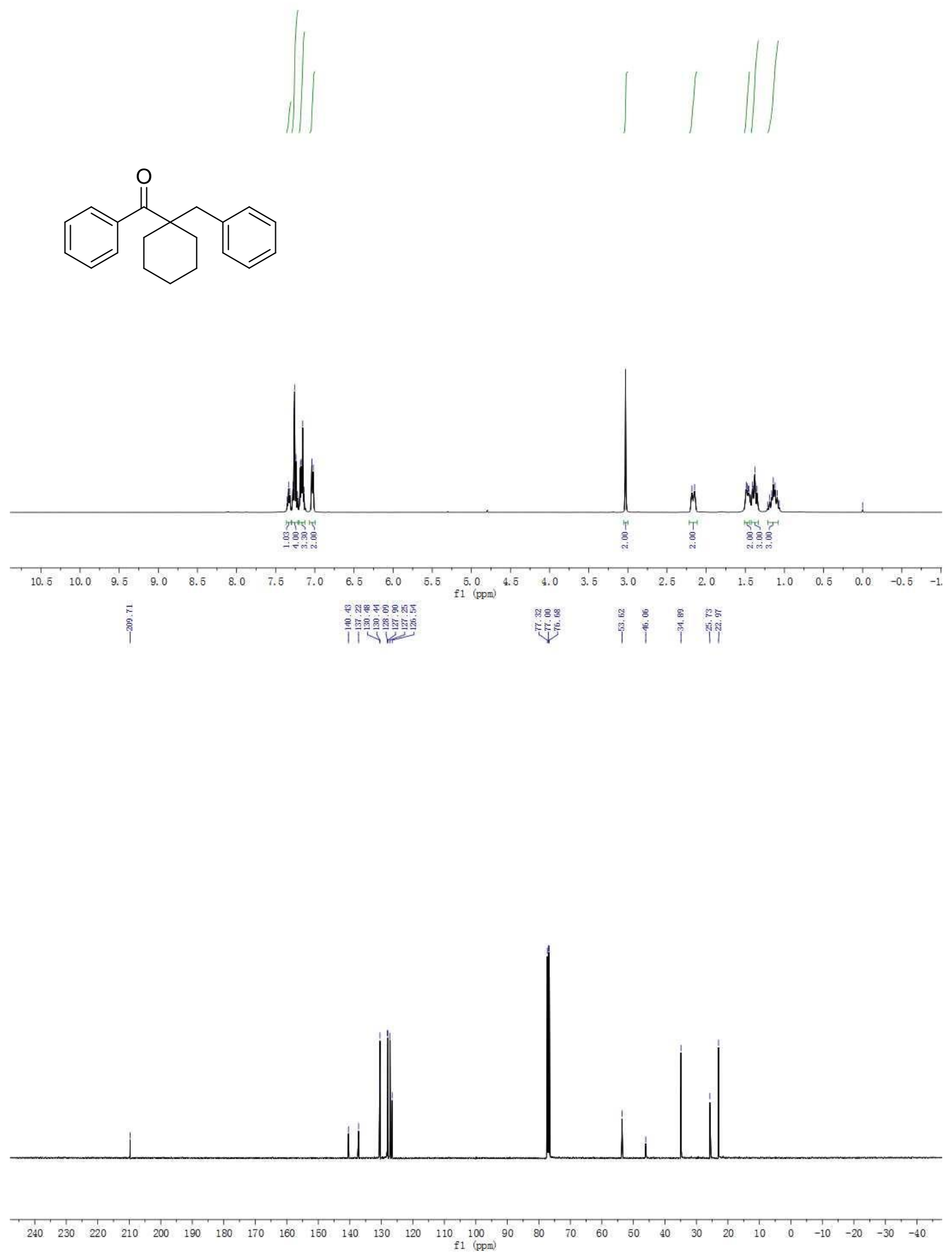

S71 
2,2-Dimethyl-3-phenyl-1-(4-vinylphenyl)propan-1-one (3m)

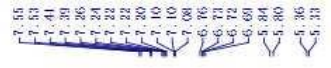
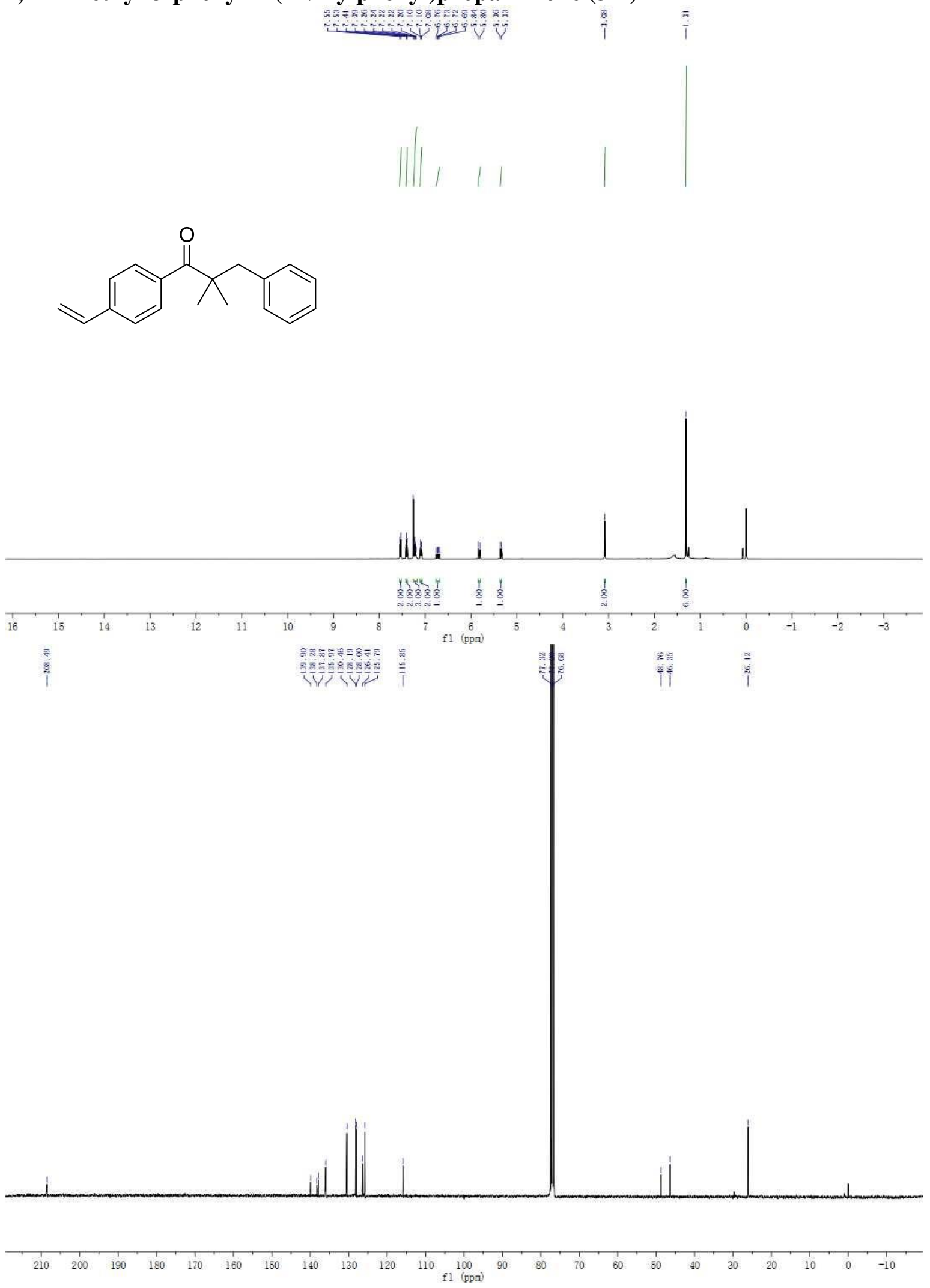

S72 
4,4-Dimethyl-1,5-diphenylpent-1-yn-3-one (3n)

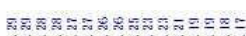

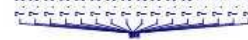
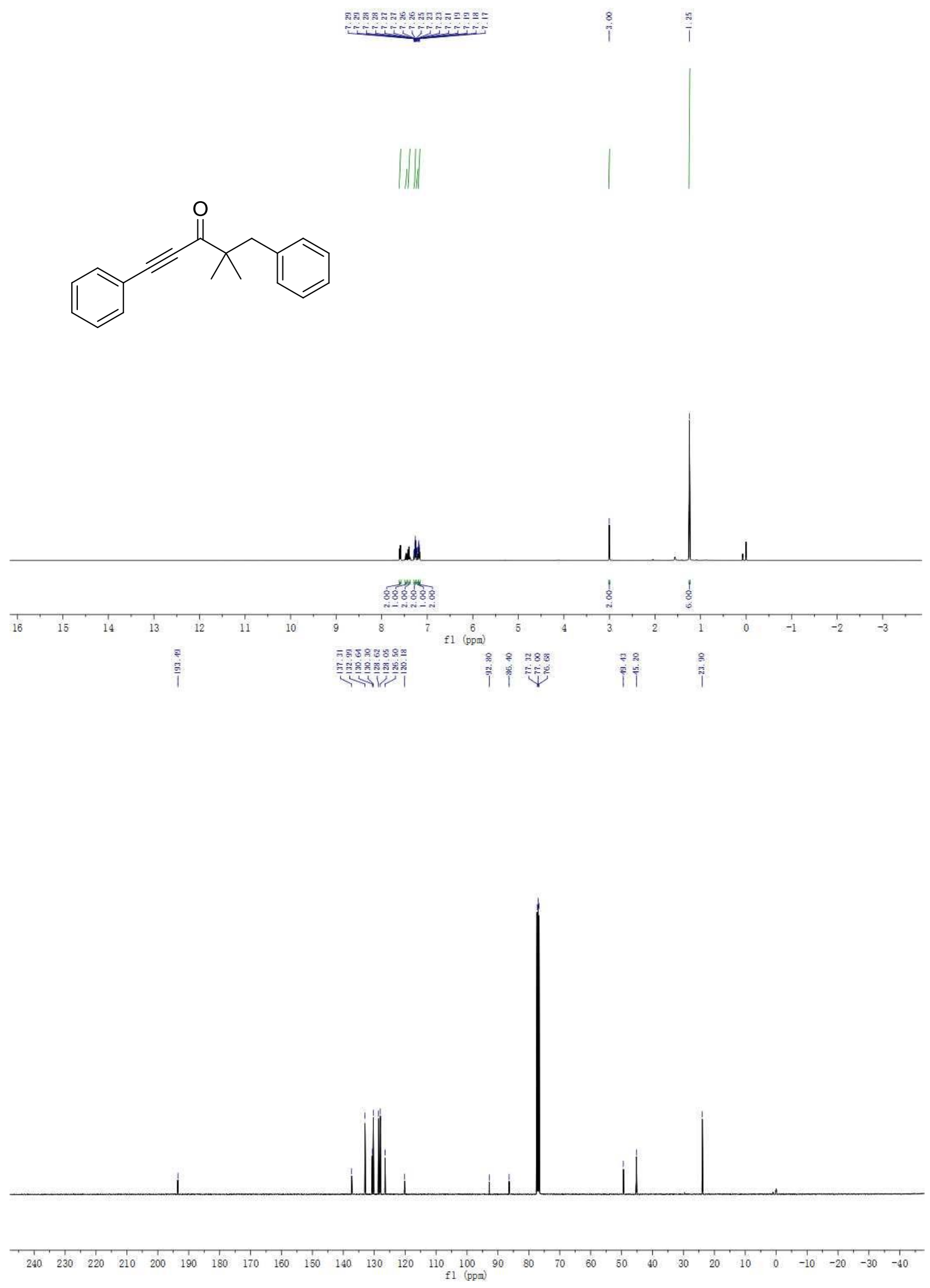

S73 
2,2-Dimethyl-3-phenyl-1-(thiophen-2-yl)propan-1-one (3o)

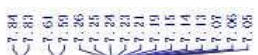
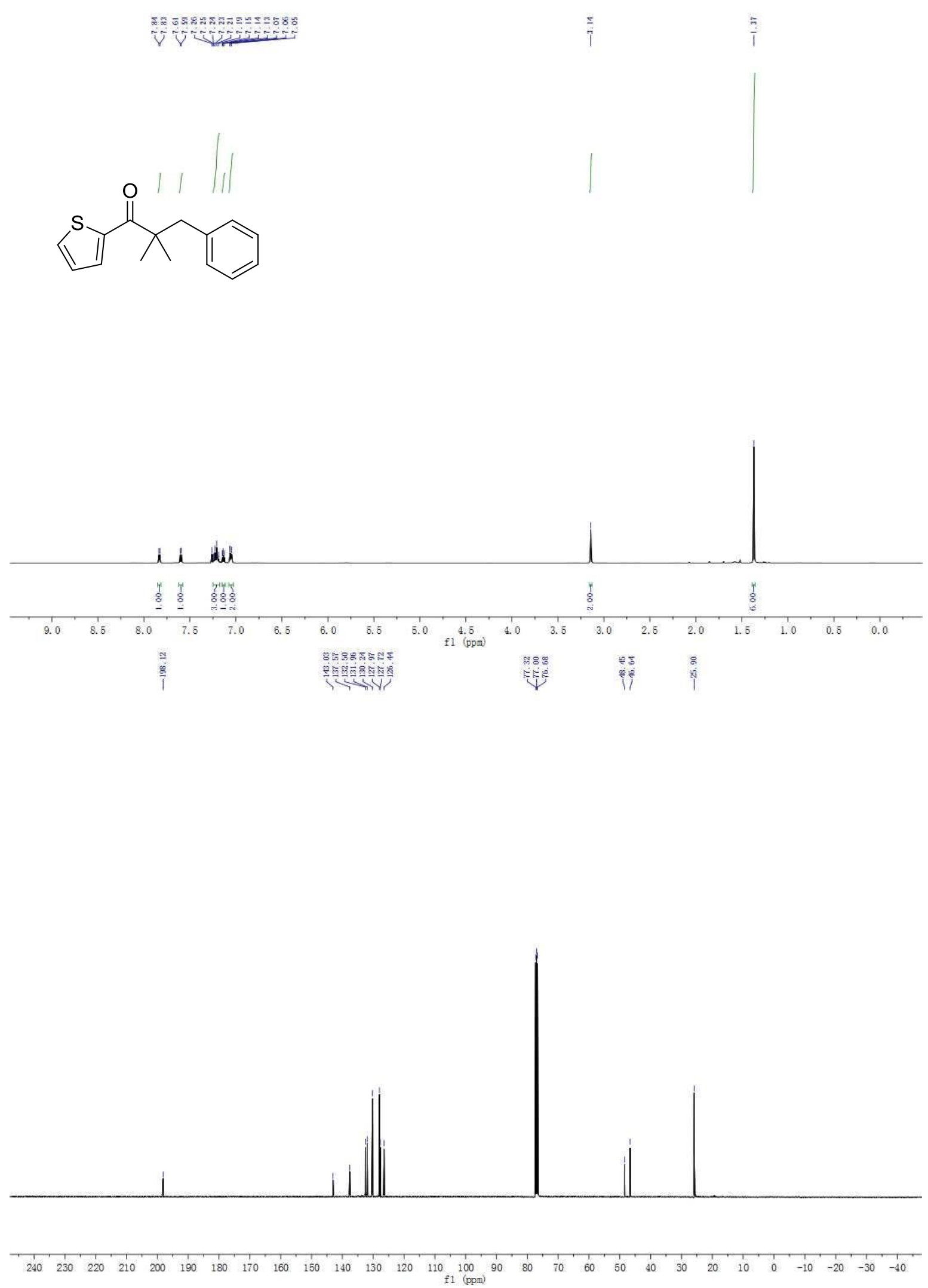

S74 
2,2-Dimethyl-3-phenyl-1-(thiophen-3-yl)propan-1-one (3p)

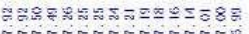
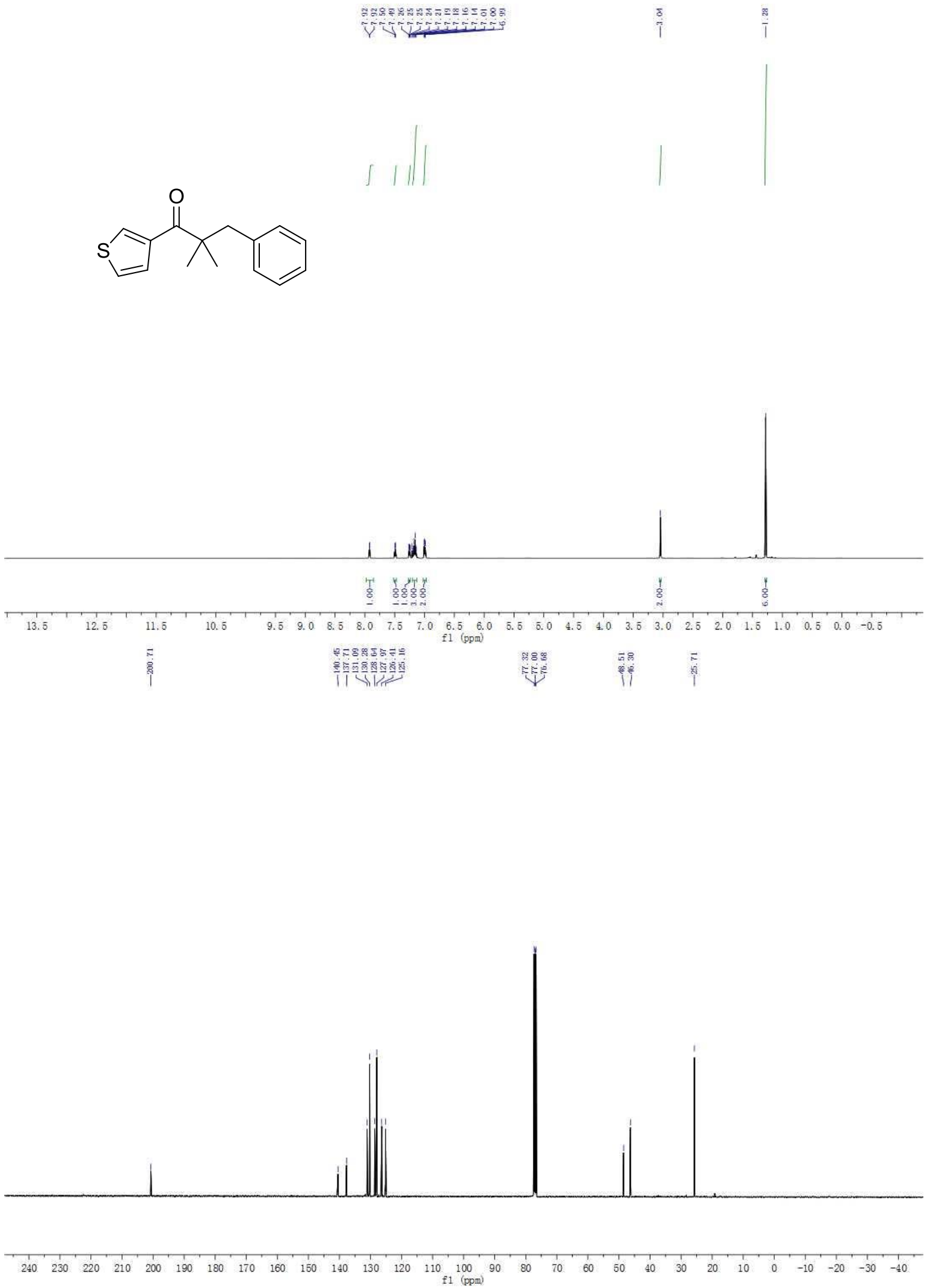

S75 
Phenyl 2,2-dimethyl-3-phenylpropanoate (3q)
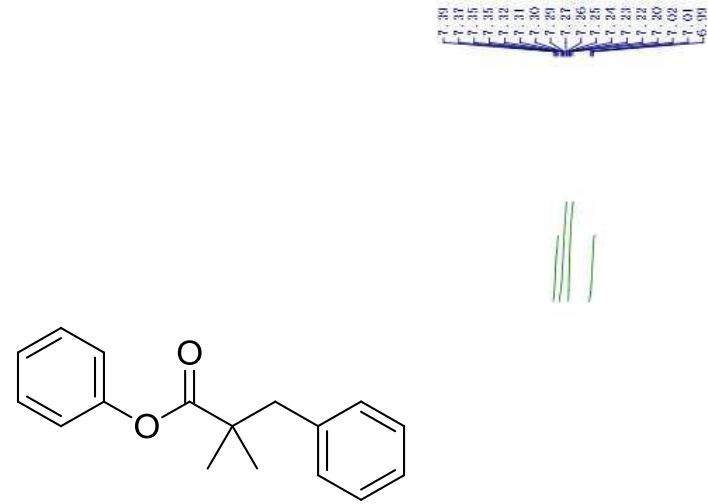
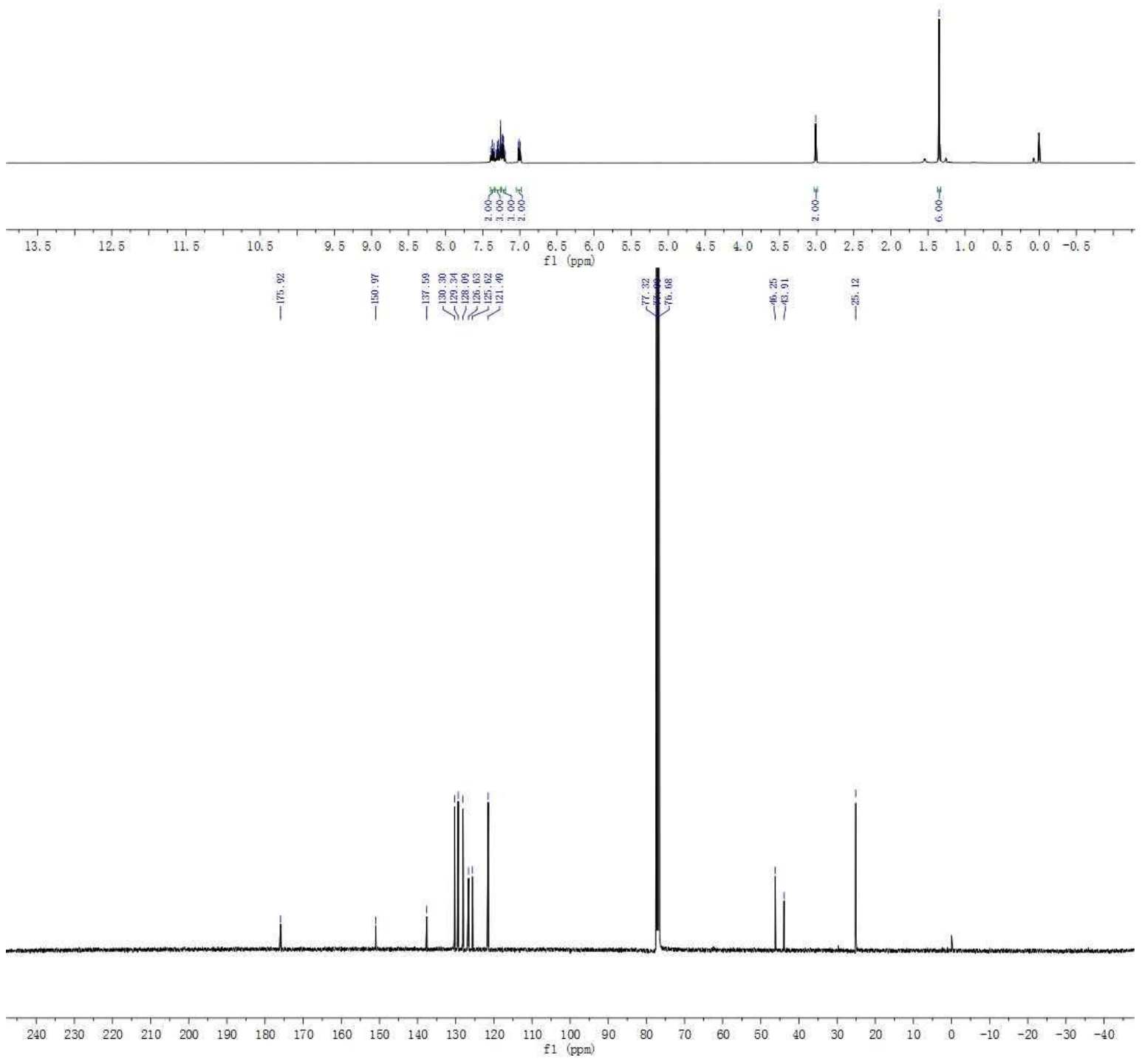

S76 
4-Acetylphenyl 2,2-dimethyl-3-phenylpropanoate (3r)
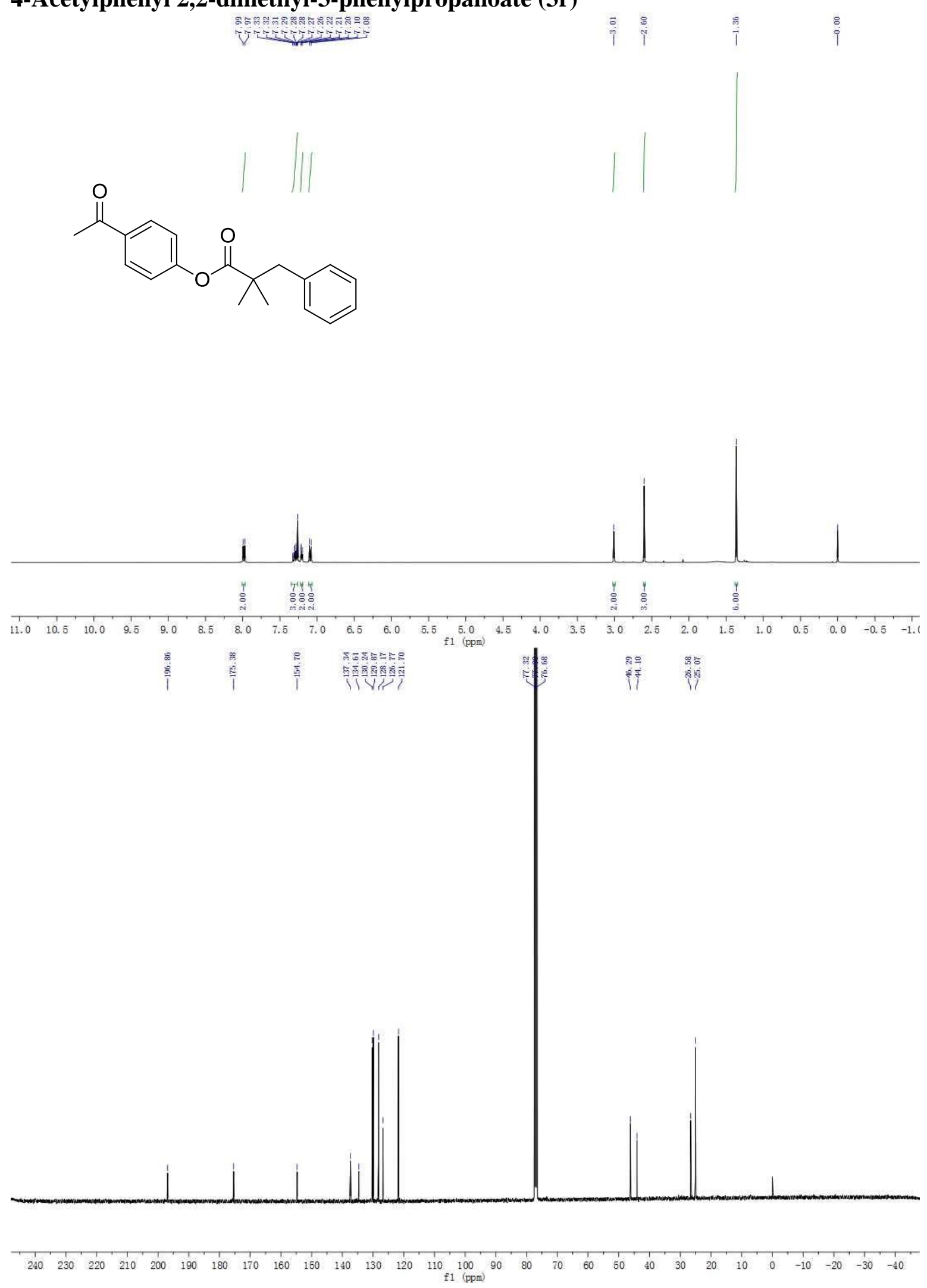

S77 
2-Benzyl-2-methyl-1-phenylbutan-1-one (3s)

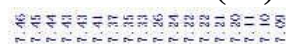

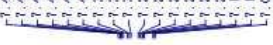
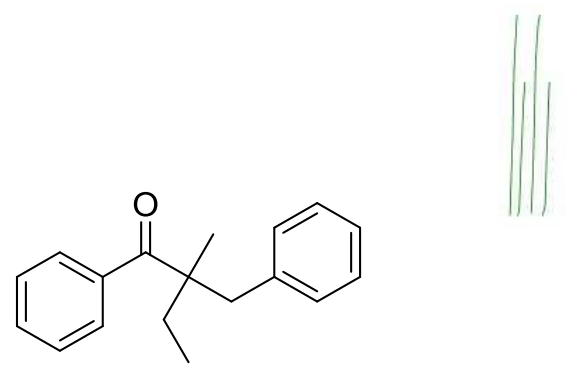

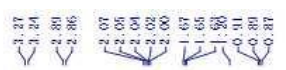

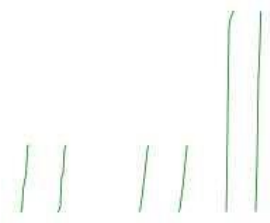

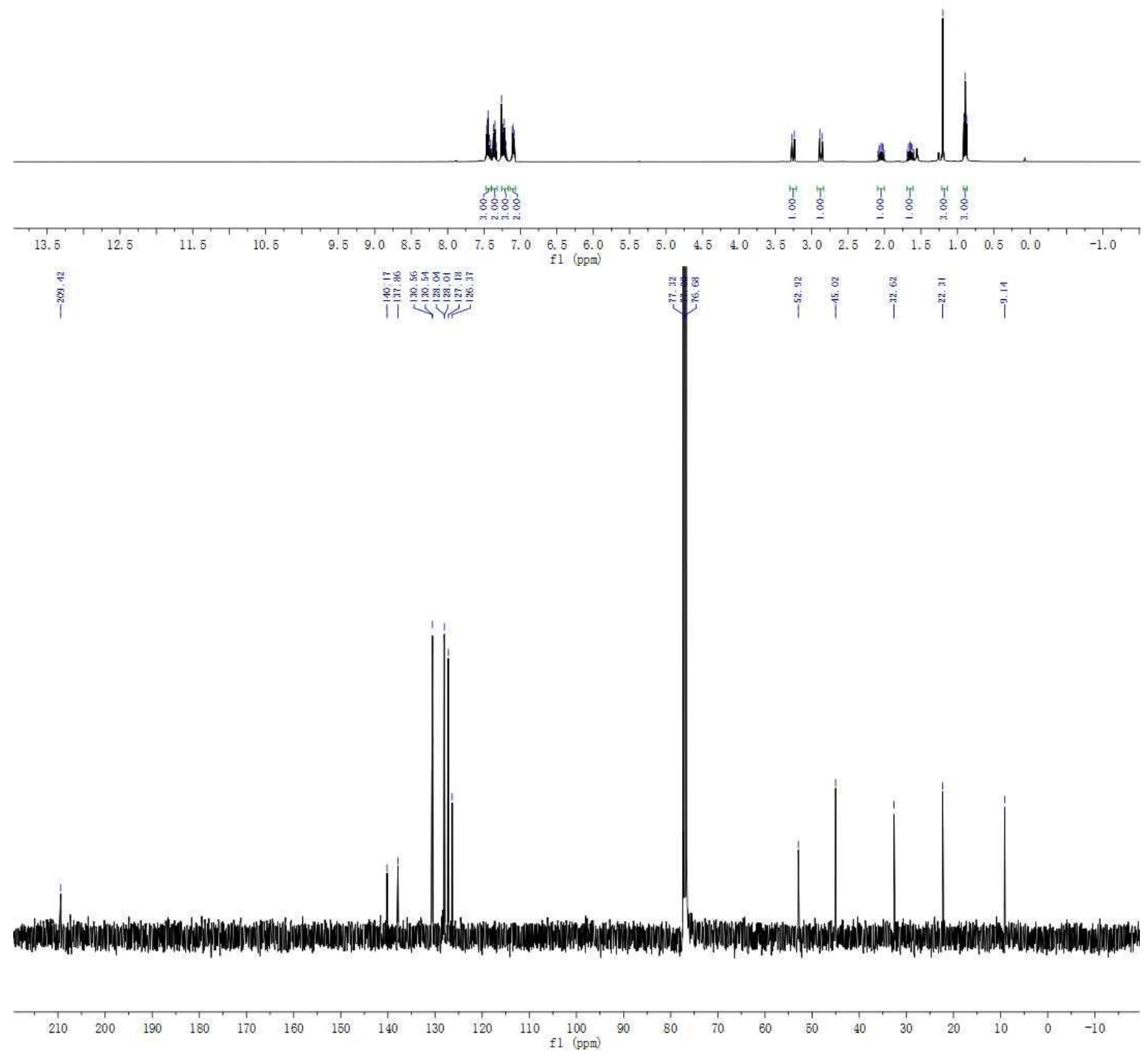

S78 
2-Benzyl-2-ethyl-1-phenylbutan-1-one (3t)

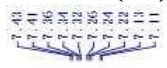

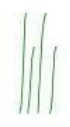

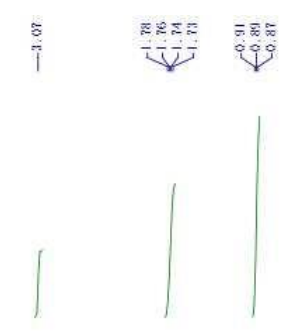
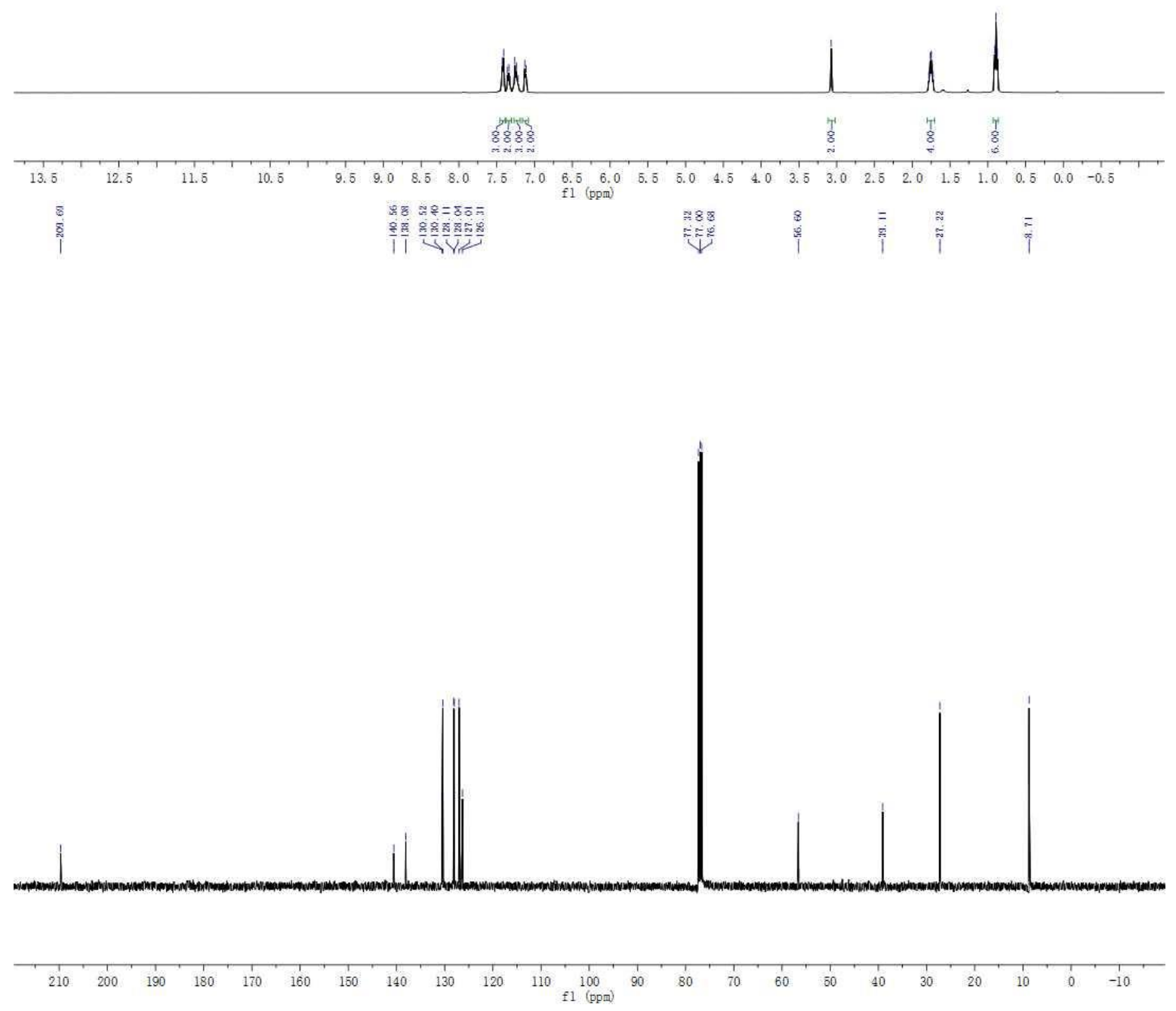

S79 
2-Benzyl-2-methylcyclohexan-1-one (3u)

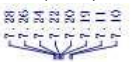

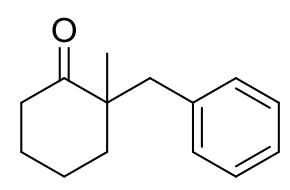

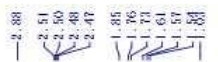

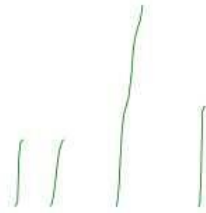

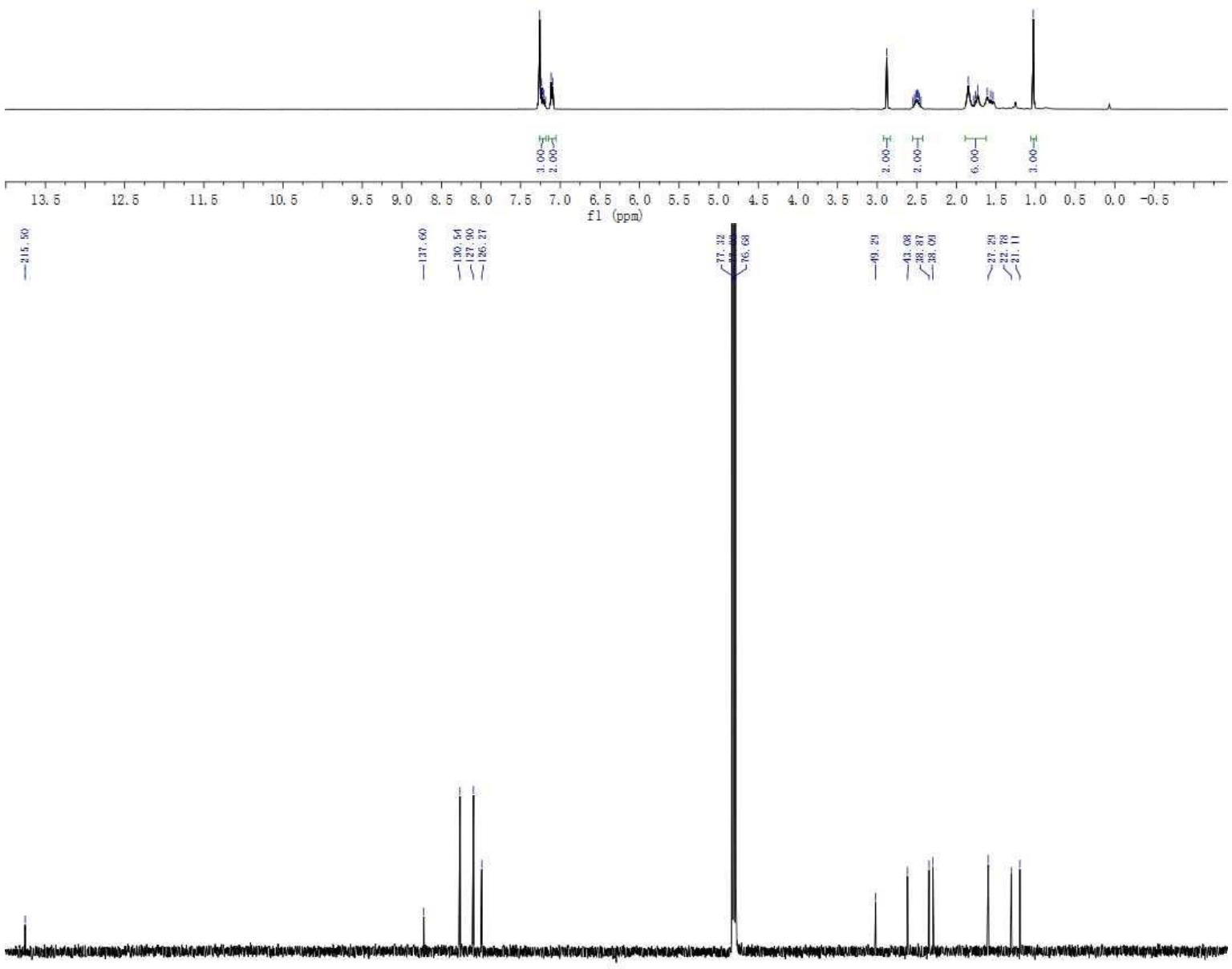

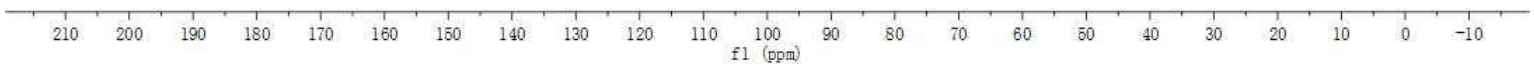

S80 
Ethyl 2-benzyl-2-methyl-3-oxo-3-phenylpropanoate (3v)

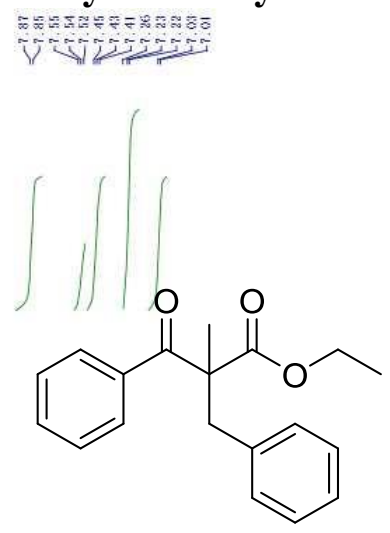

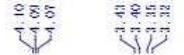

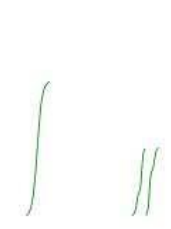

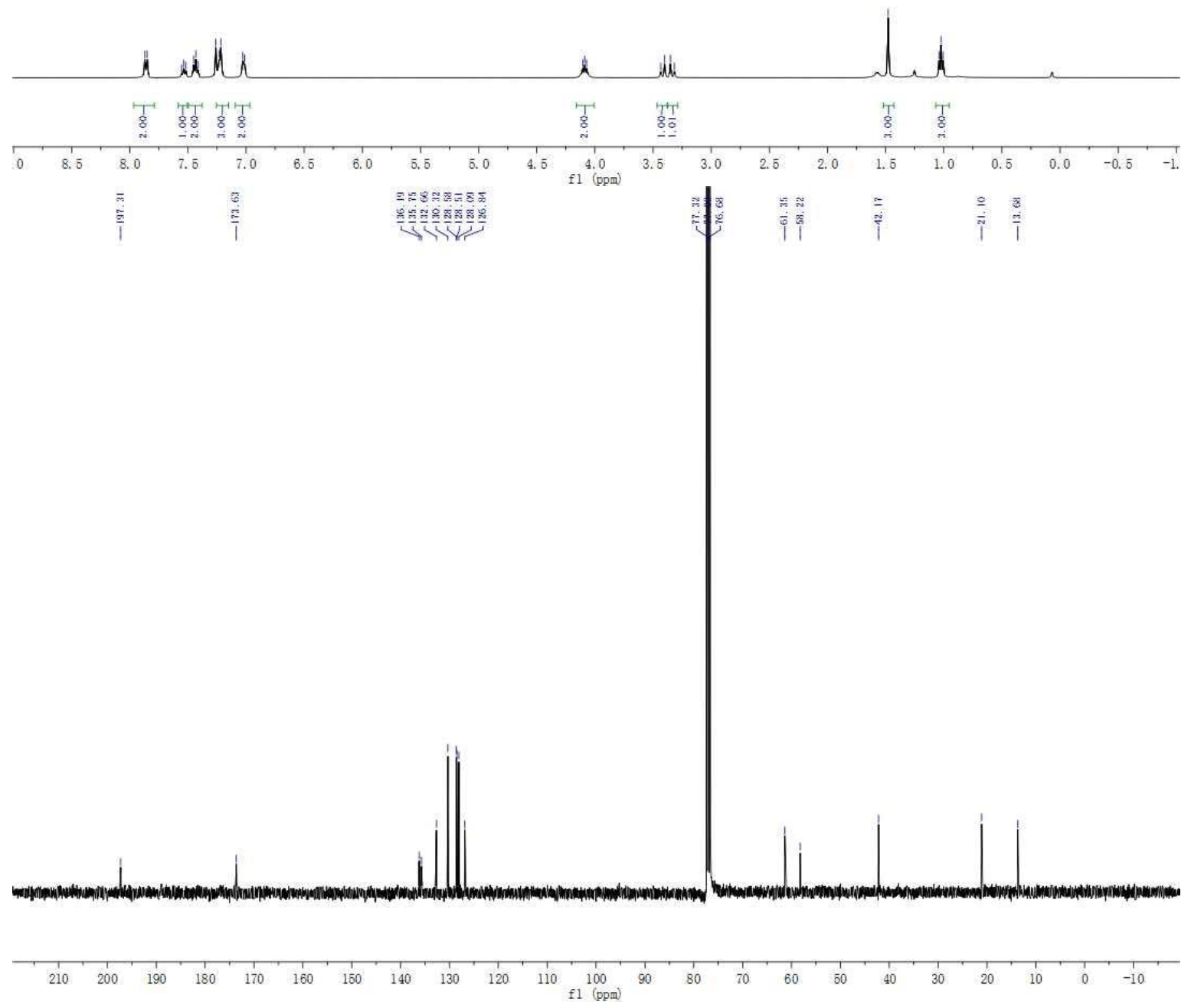

S81 
2-Benzyl-3-methoxy-2-methyl-1-phenylpropan-1-one (3x)

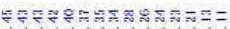

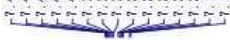

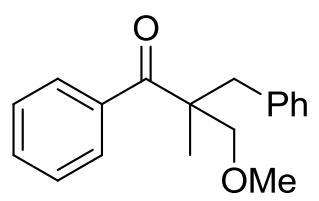

죽농ำ유유 $=558$

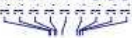

$\frac{5}{1}$
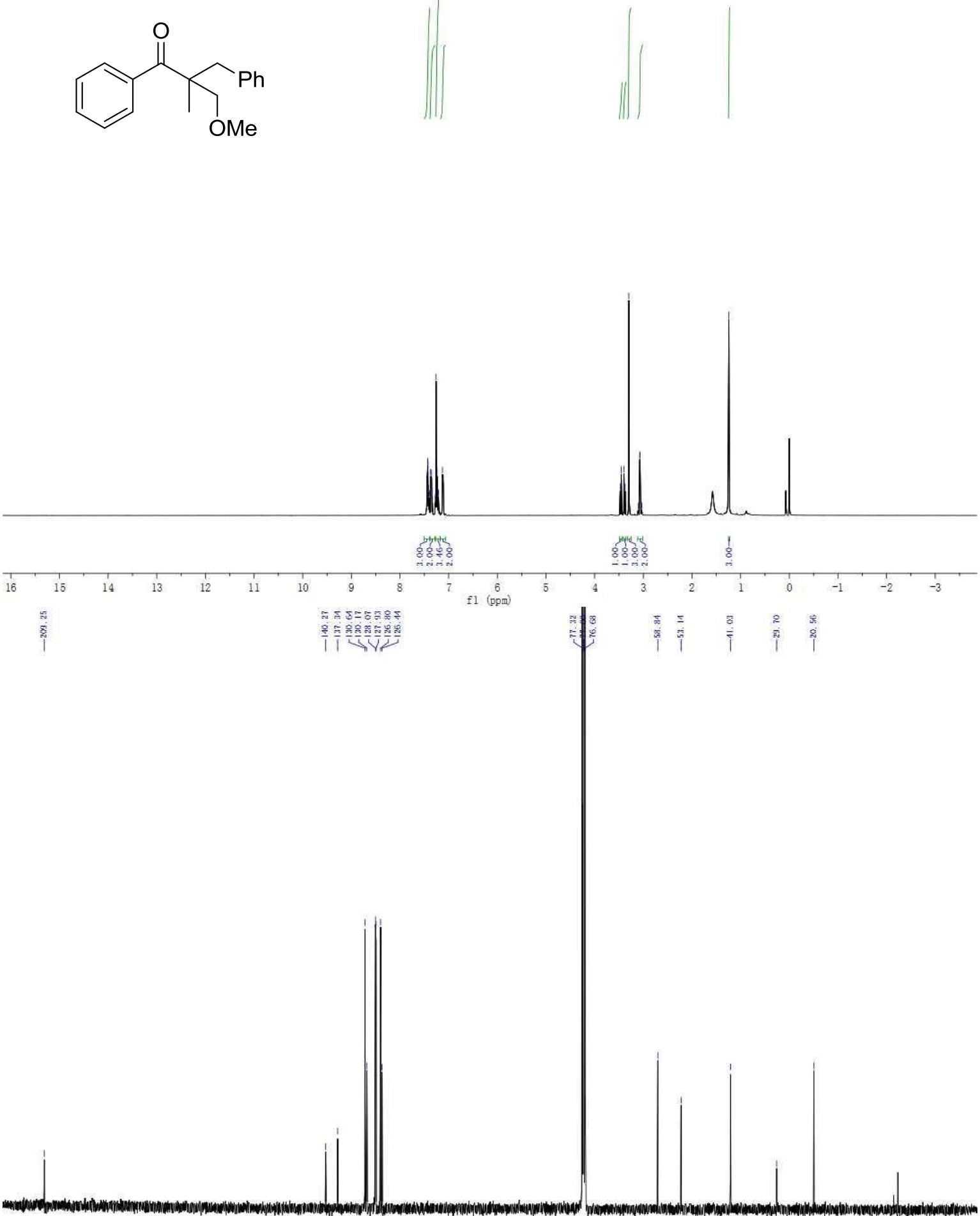

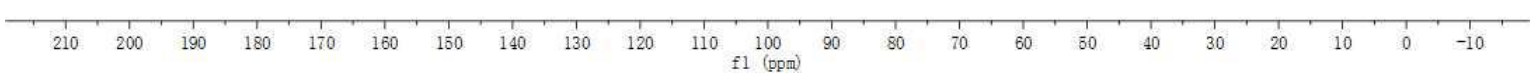

S82 
3-(4-Methoxyphenyl)-2,2-dimethyl-1-phenylpropan-1-one (3y)

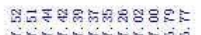

isinitisis
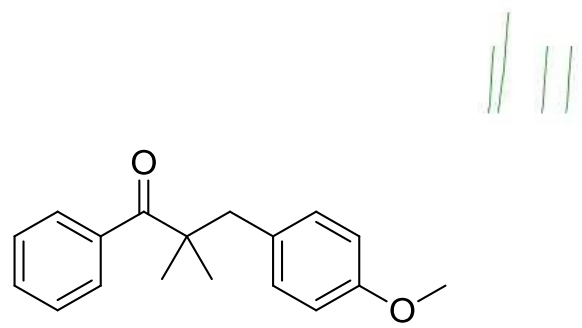

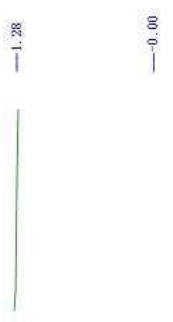

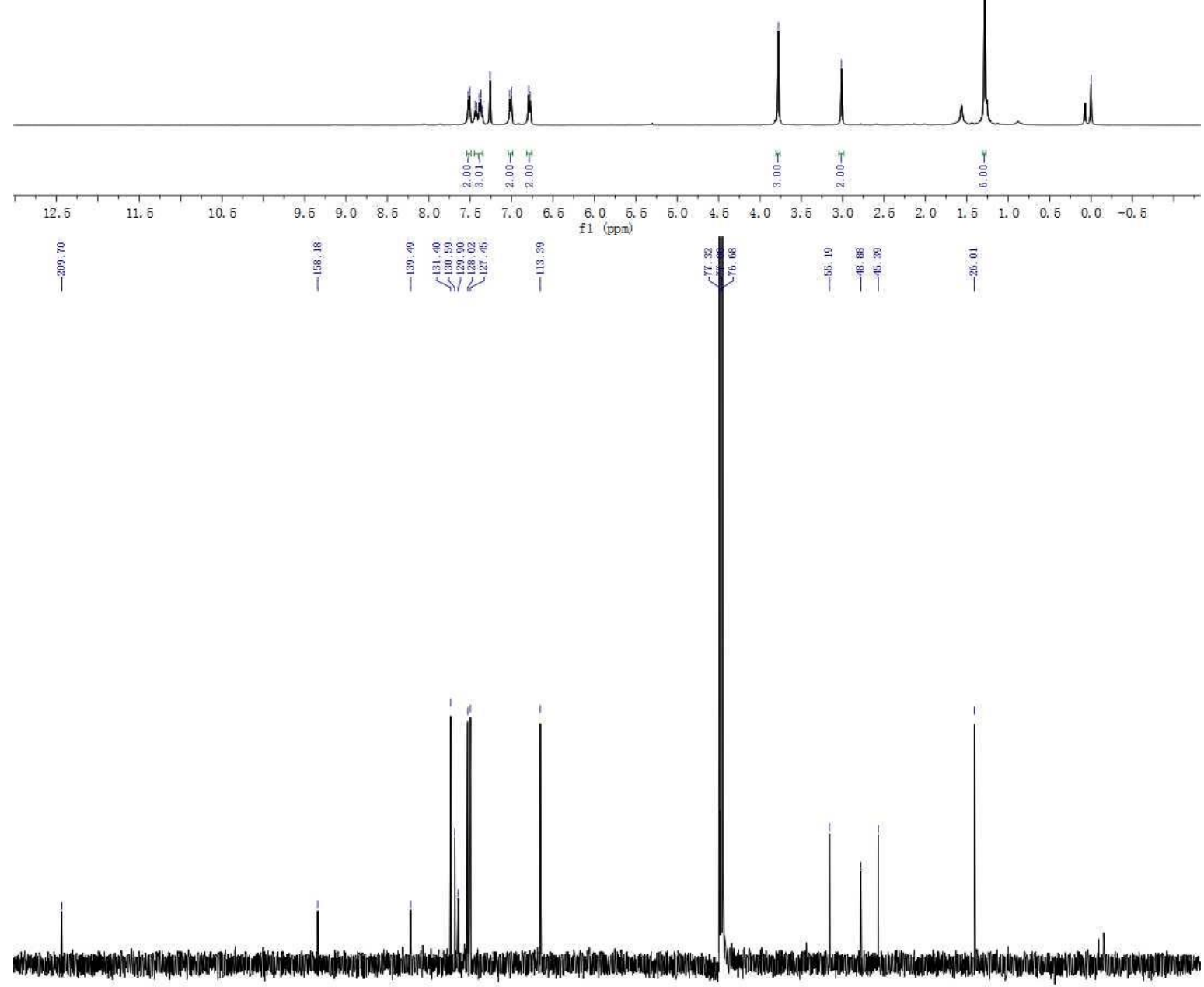

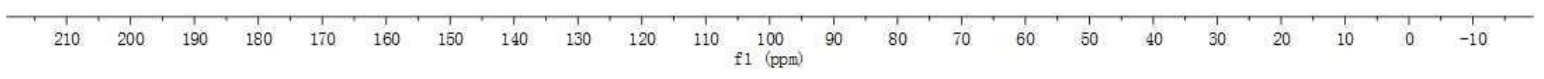

S83 
1-(4-Fluorophenyl)-3-(4-methoxyphenyl)-2,2-dimethylpropan-1-one (3z) 589 vivitiogist

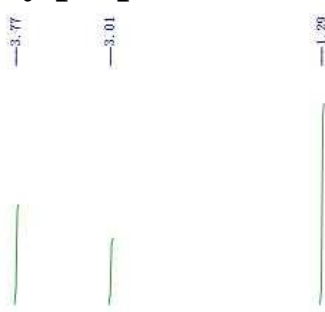<smiles>COc1ccc(CC(C)(C)C(=O)c2ccc(F)cc2)cc1</smiles>

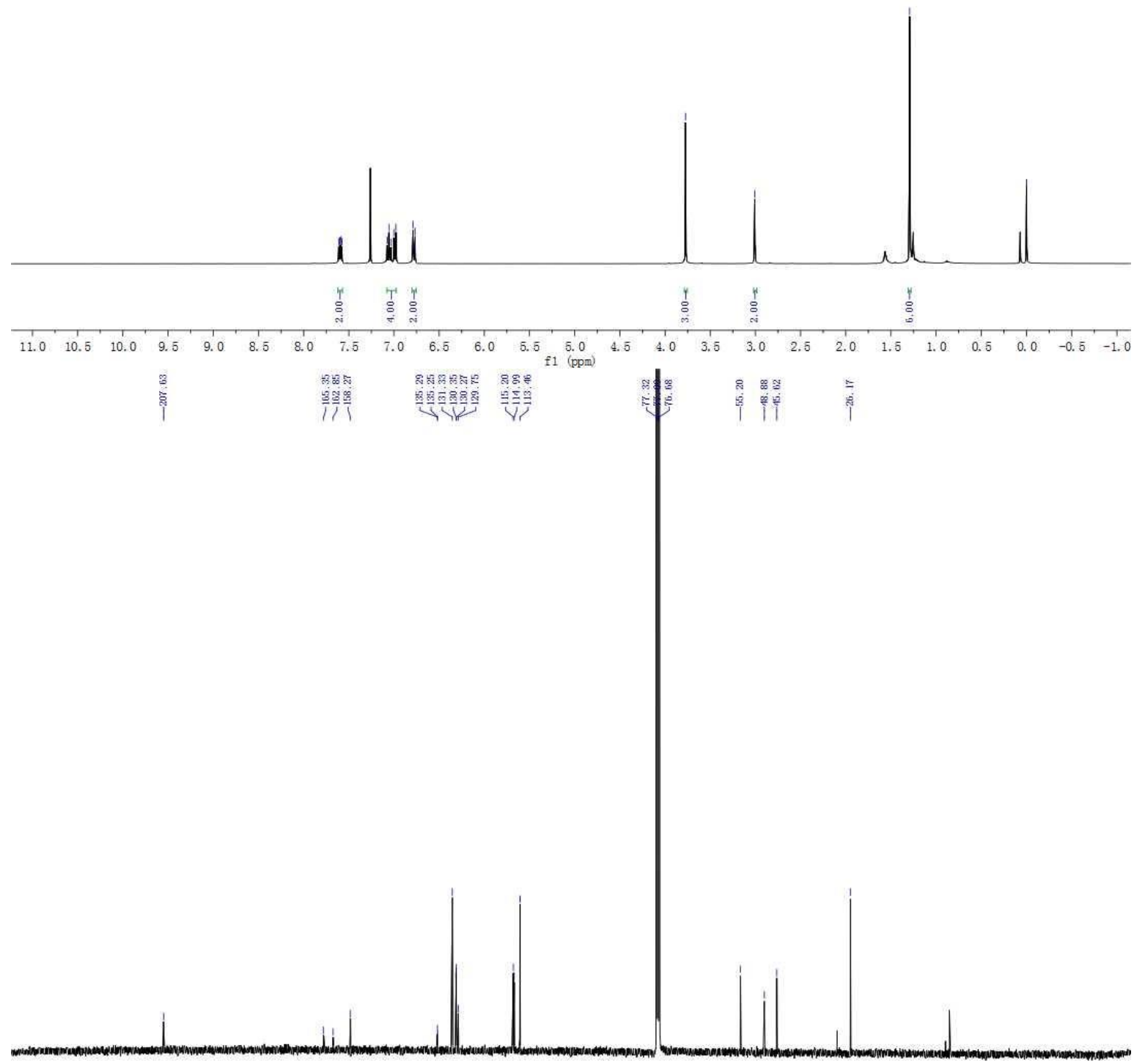

$\begin{array}{lllllllllllllllllllllllllllllllllllllllll}240 & 230 & 220 & 210 & 200 & 190 & 180 & 170 & 160 & 150 & 140 & 130 & 120 & 110 & 100 & 90 & 80 & 70 & 60 & 50 & 40 & 30 & 20 & 10 & 0 & -10 & -20 & -30 & -40\end{array}$ 
1-Mesityl-3-(4-methoxyphenyl)-2,2-dimethylpropan-1-one (3aa)
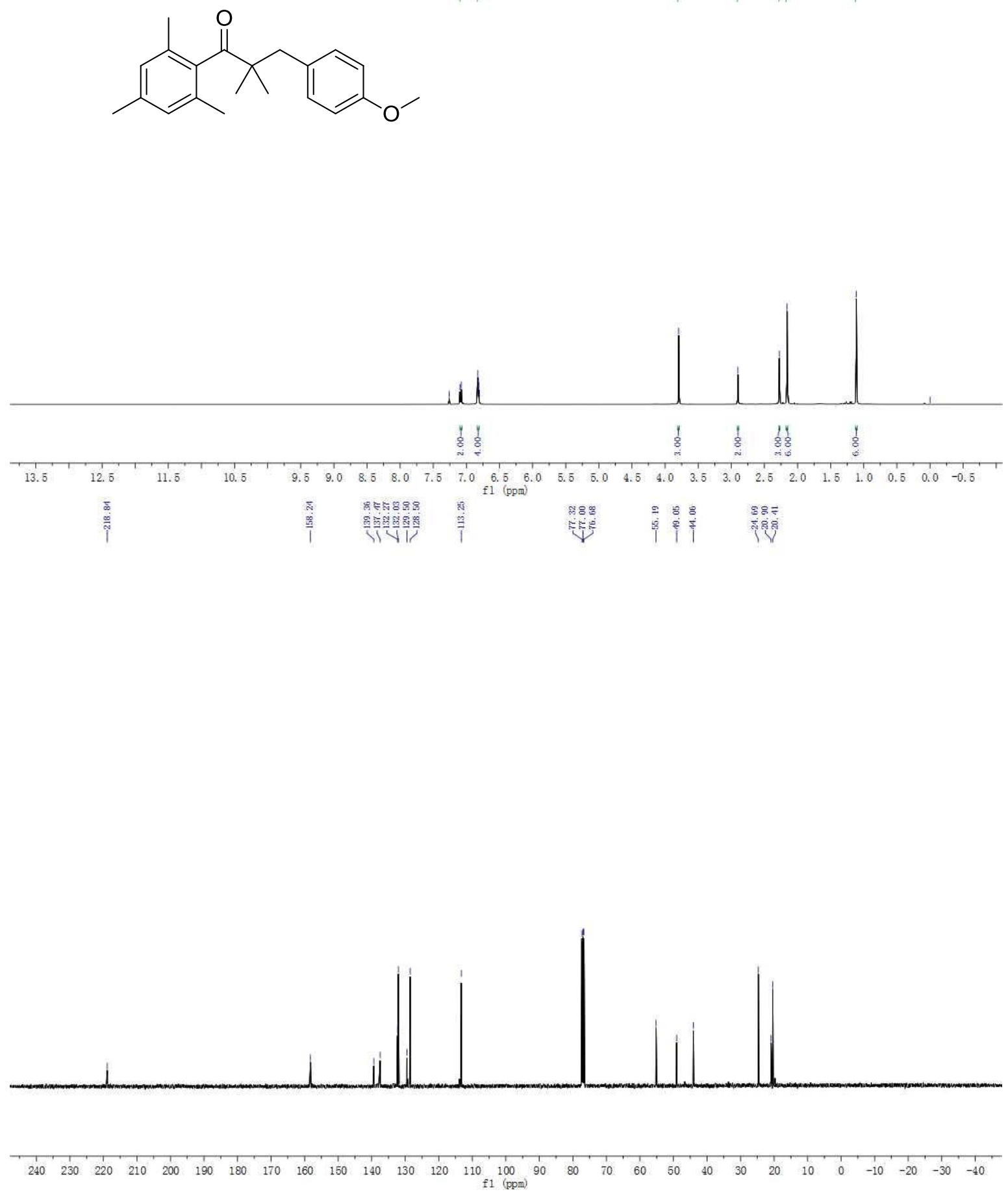

S85 
3-(4-Methoxyphenyl)-2,2-dimethyl-1-(thiophen-2-yl)propan-1-one (3ab)

중 8 문
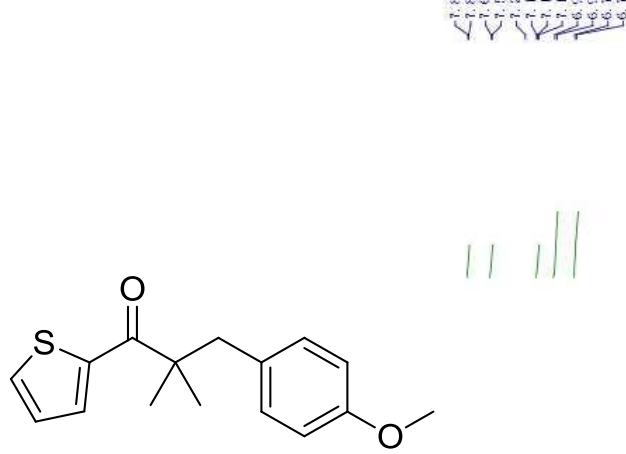
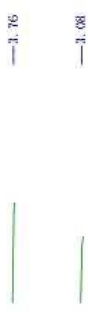
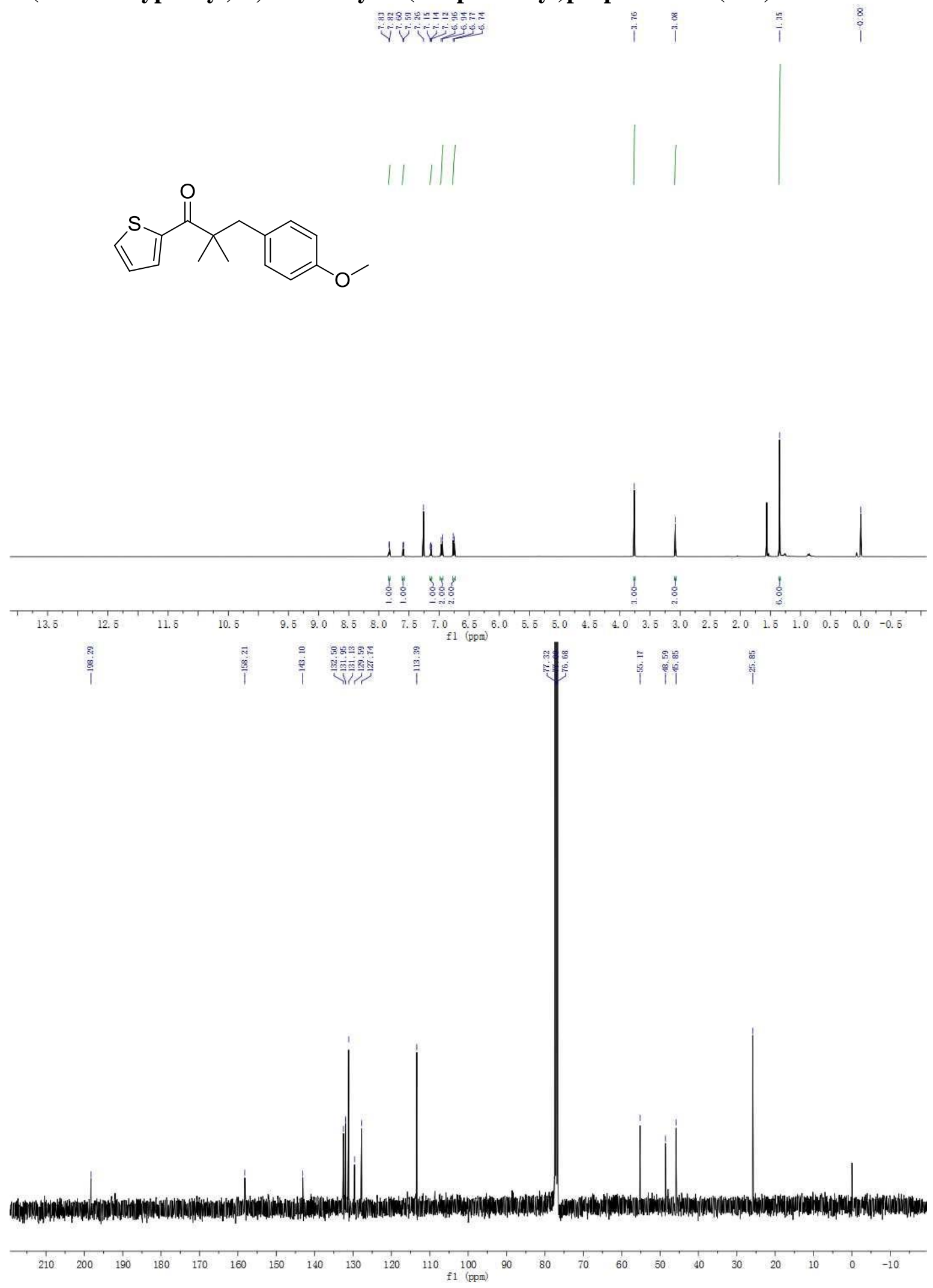

S86 
3-(4-Chlorophenyl)-2,2-dimethyl-1-phenylpropan-1-one (3ac)
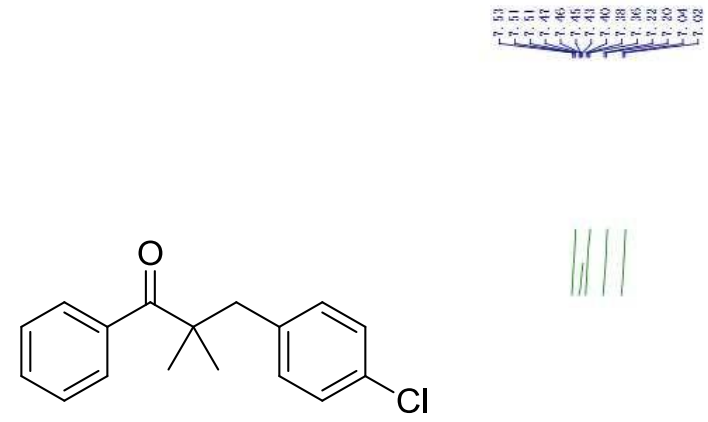

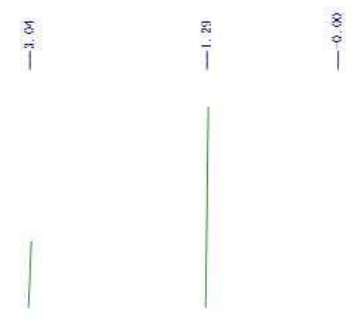

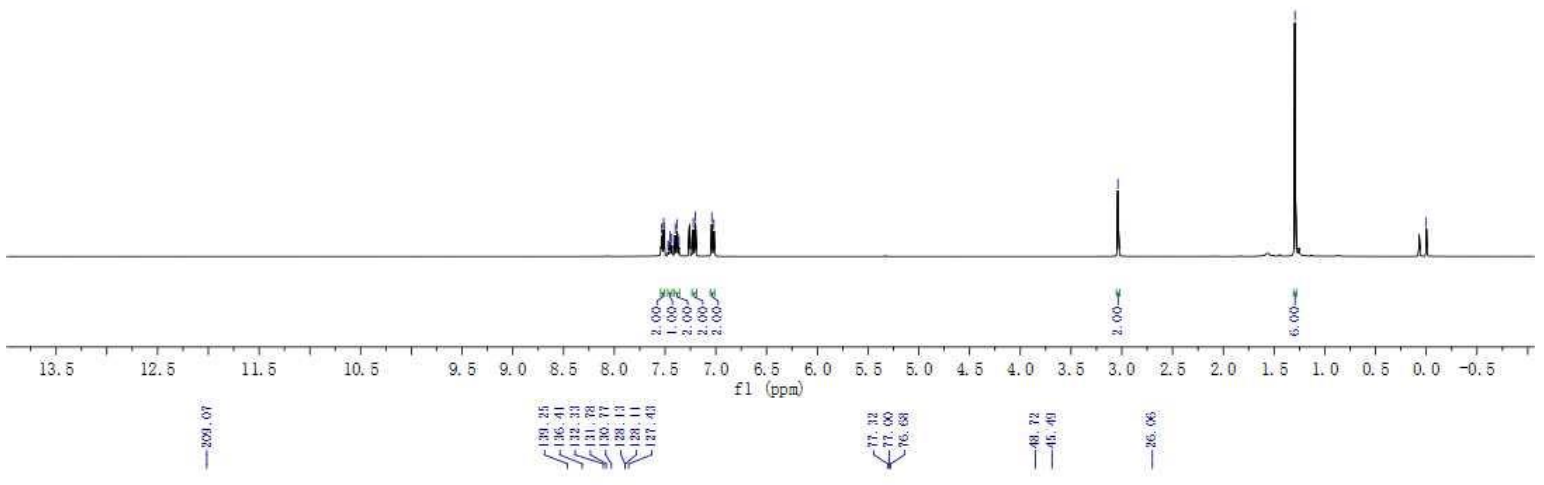
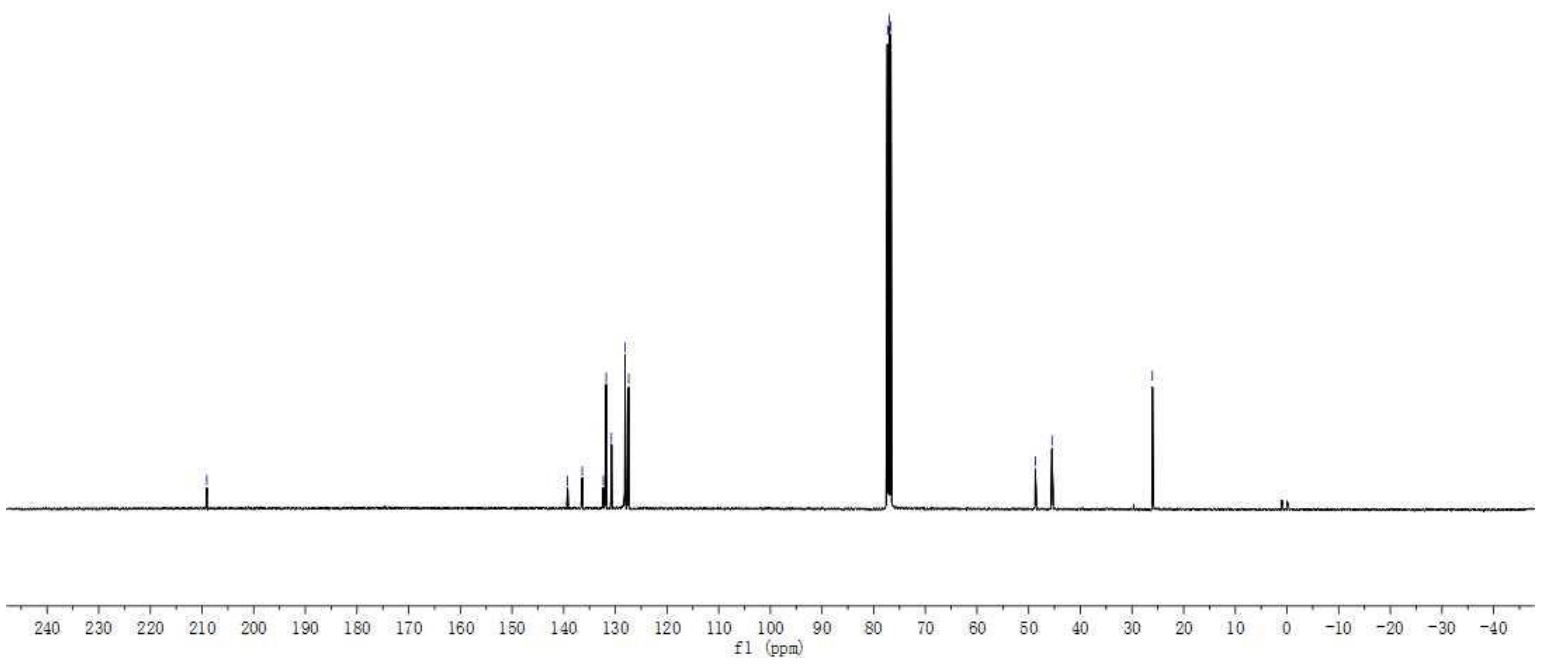

S87 
3-(4-Chlorophenyl)-2,2-dimethyl-1-(thiophen-2-yl)propan-1-one (3ad)

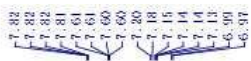
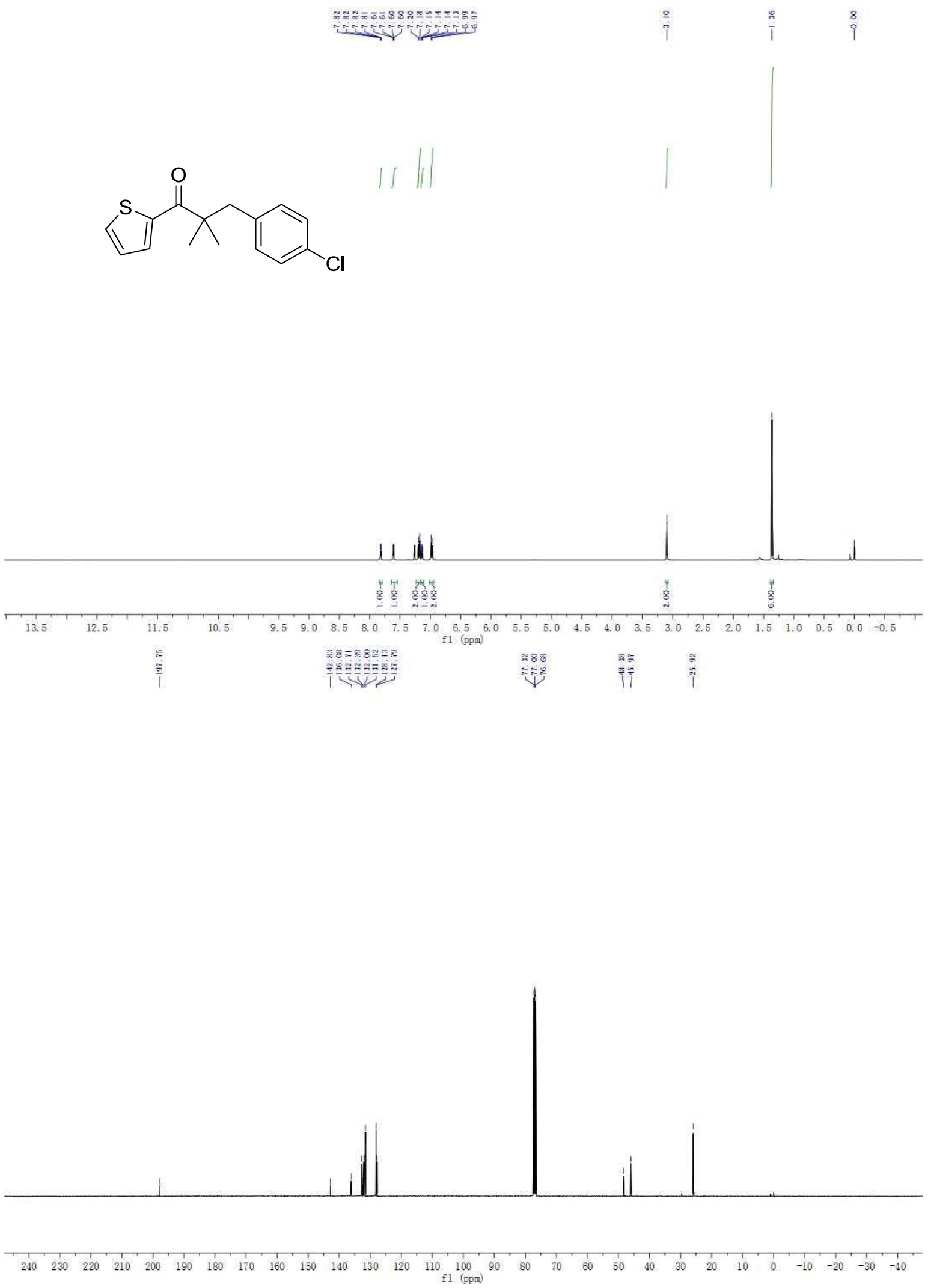

S88 
2,2-Dimethyl-1-phenyl-3-(thiophen-2-yl)propan-1-one (3ae)

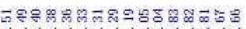

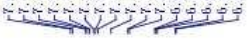<smiles>CC(C)(Cc1cccs1)C(=O)c1ccccc1</smiles>
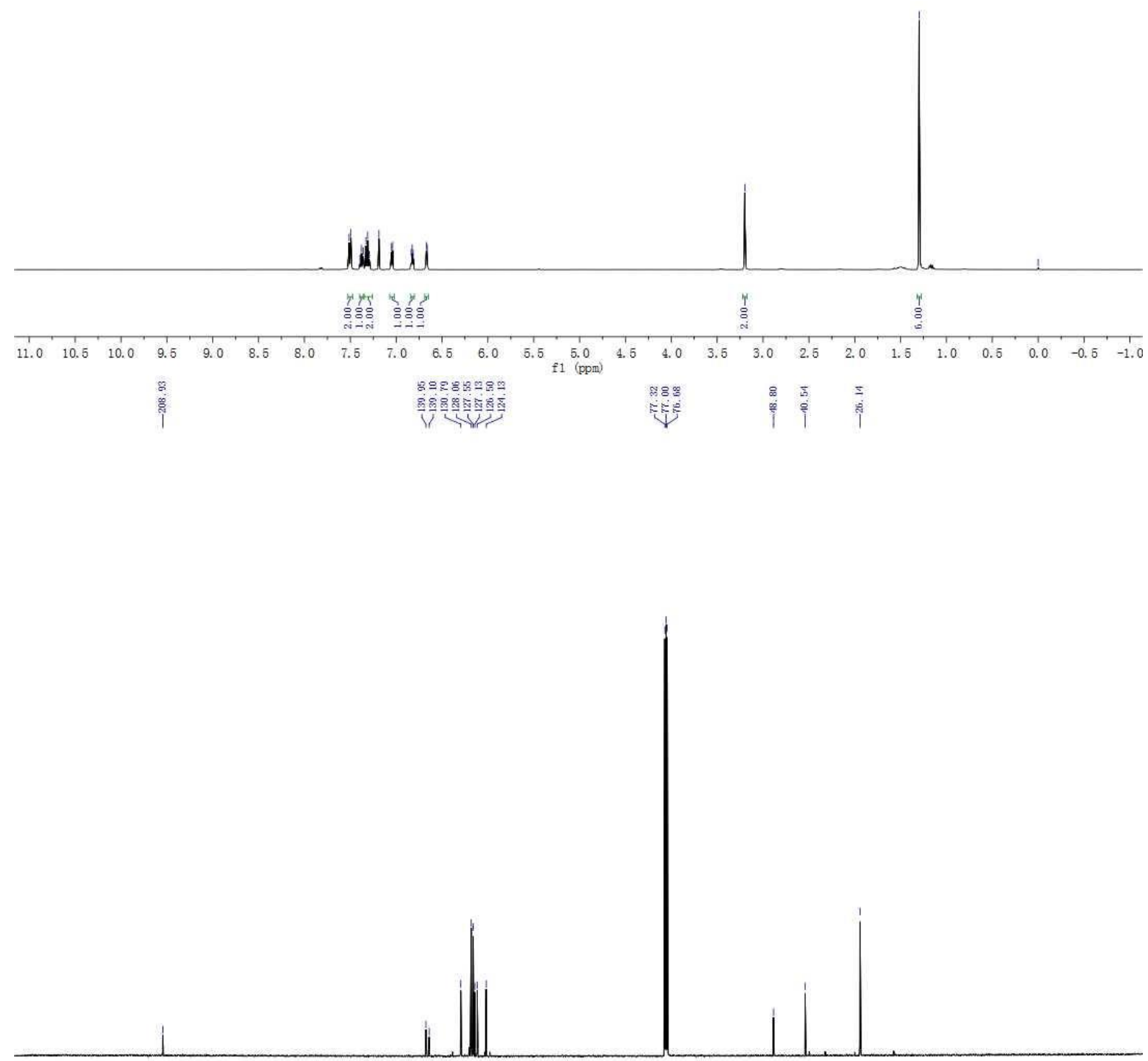

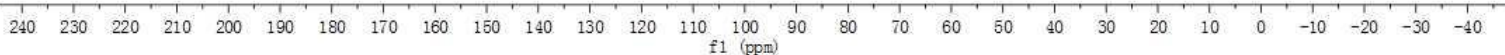


2,2-Dimethyl-1,3-diphenylbutan-1-one (3af)
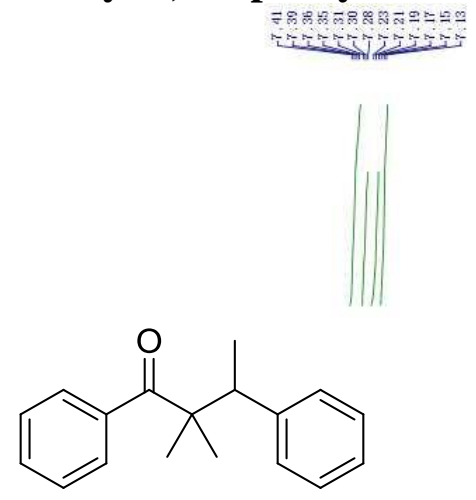

50959
ning

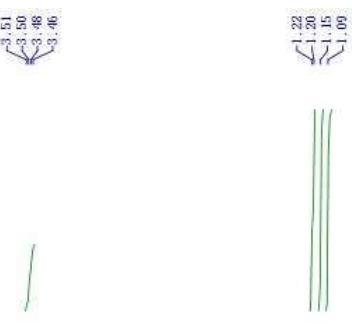

$\stackrel{8}{1}$
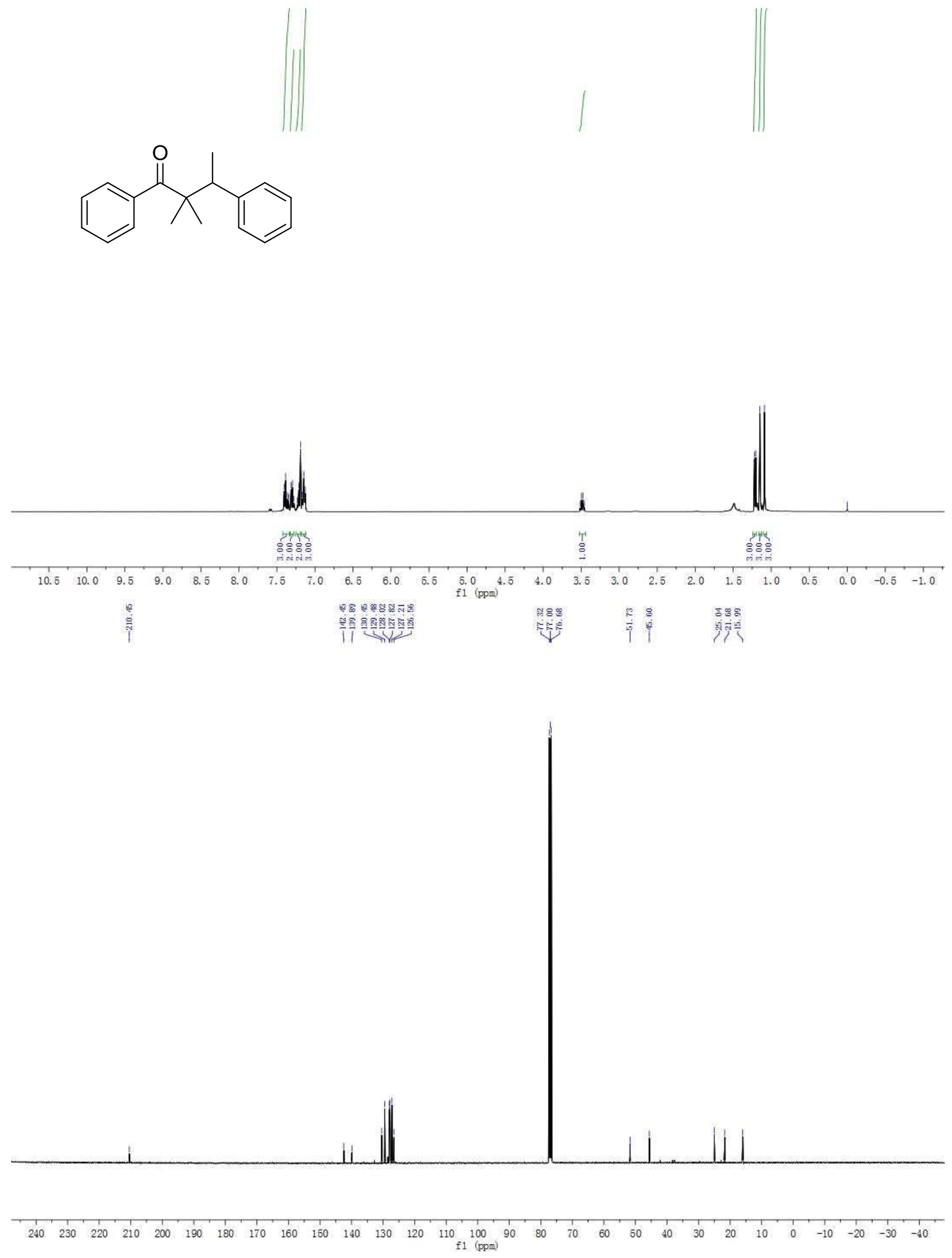

S90 
1-(3-Chlorophenyl)-2,2-dimethyl-3-phenylbutan-1-one (3ag)

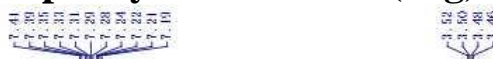

$\begin{array}{ll}9 \times 2 \approx=5 & 8 \\ \text { पit } & \text { i }\end{array}$
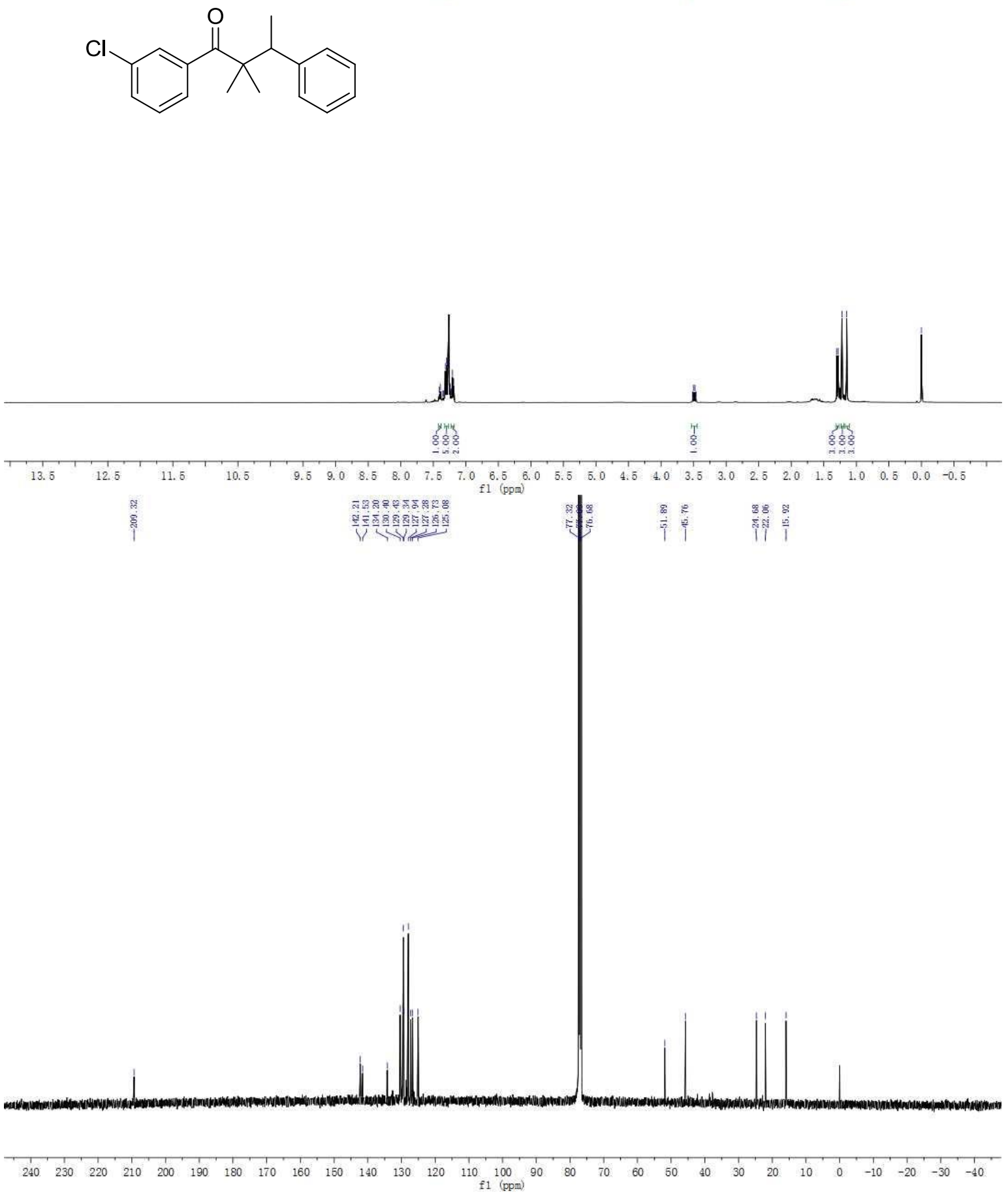

S91 
2,6-Dimethyl-4-(2-phenylpropan-2-yl)-1,4-dihydropyridine-3,5-dicarbonitrile(7a)

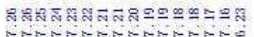
vimitastio<smiles>CC1=C(C#N)C(C(C)(C)c2ccccc2)C(C#N)=C(C)N1</smiles>

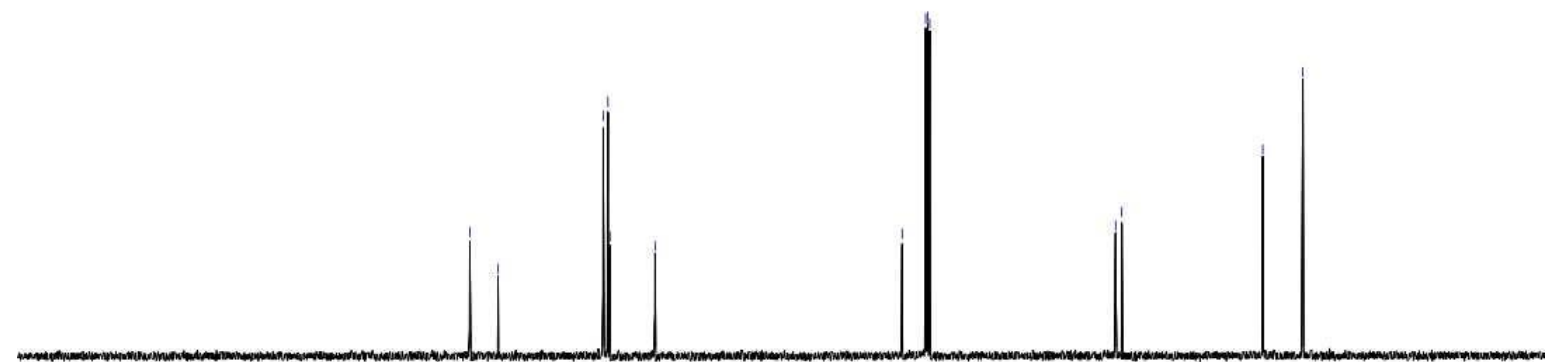
$160 \quad 140$ $130 \quad 120$ $110 \underset{f 1}{100}(\mathrm{ppm})$ 
4-(2-(4-Methoxyphenyl)propan-2-yl)-2,6-dimethyl-1,4-dihydropyridine-3,5-dicarbonitrile(7b)

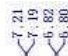<smiles>COc1ccc(C(C)(C)C2C(C#N)=C(C)NC(C)=C2C#N)cc1</smiles>

$\mathrm{CN}$
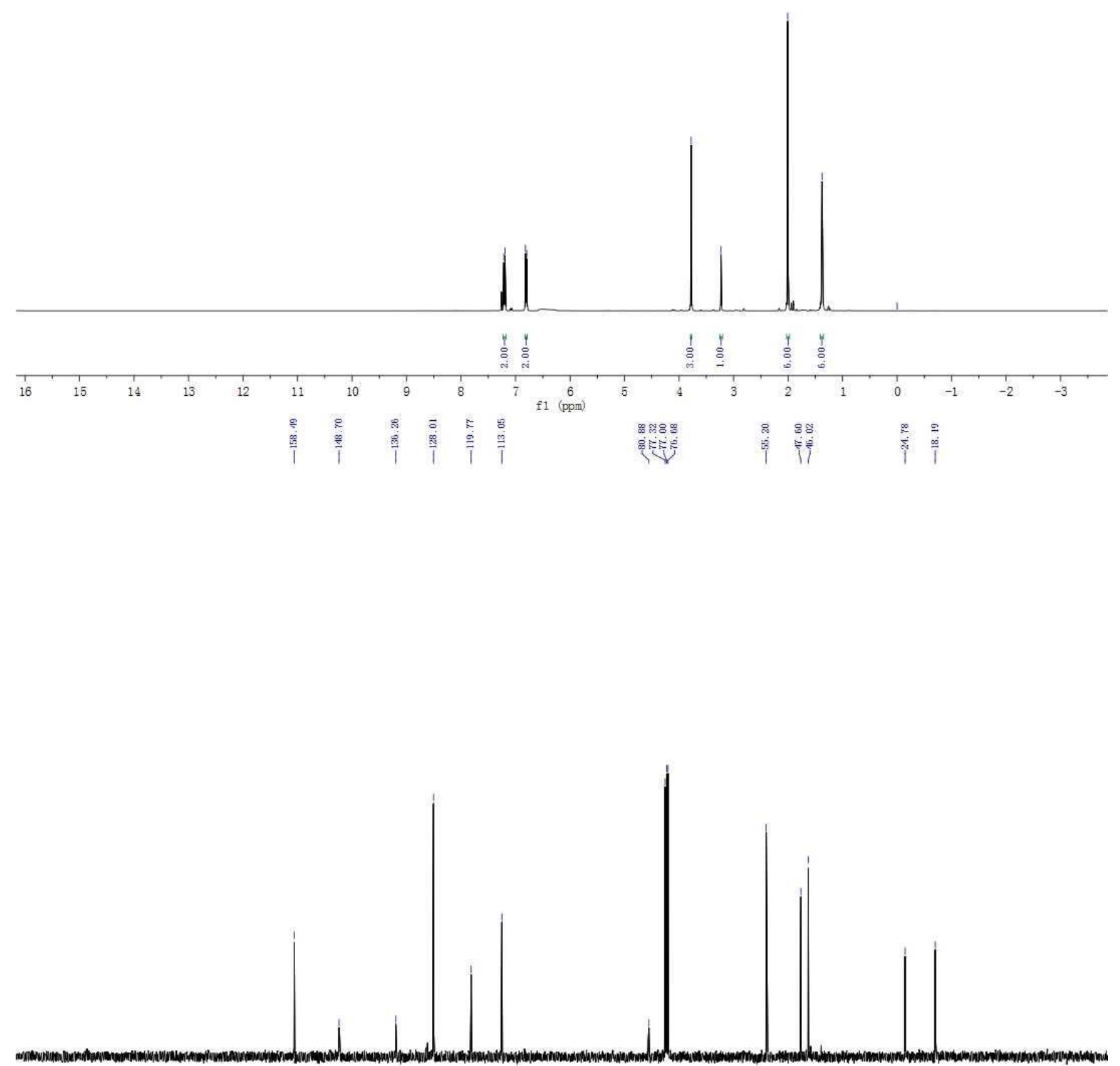
4-(2-(4-Chlorophenyl)propan-2-yl)-2,6-dimethyl-1,4-dihydropyridine-3,5-dicarbonitrile(7c)

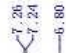

î.<smiles>CC1=C(C#N)C(C(C)(C)c2ccc(Cl)cc2)C(C#N)=C(C)N1</smiles>
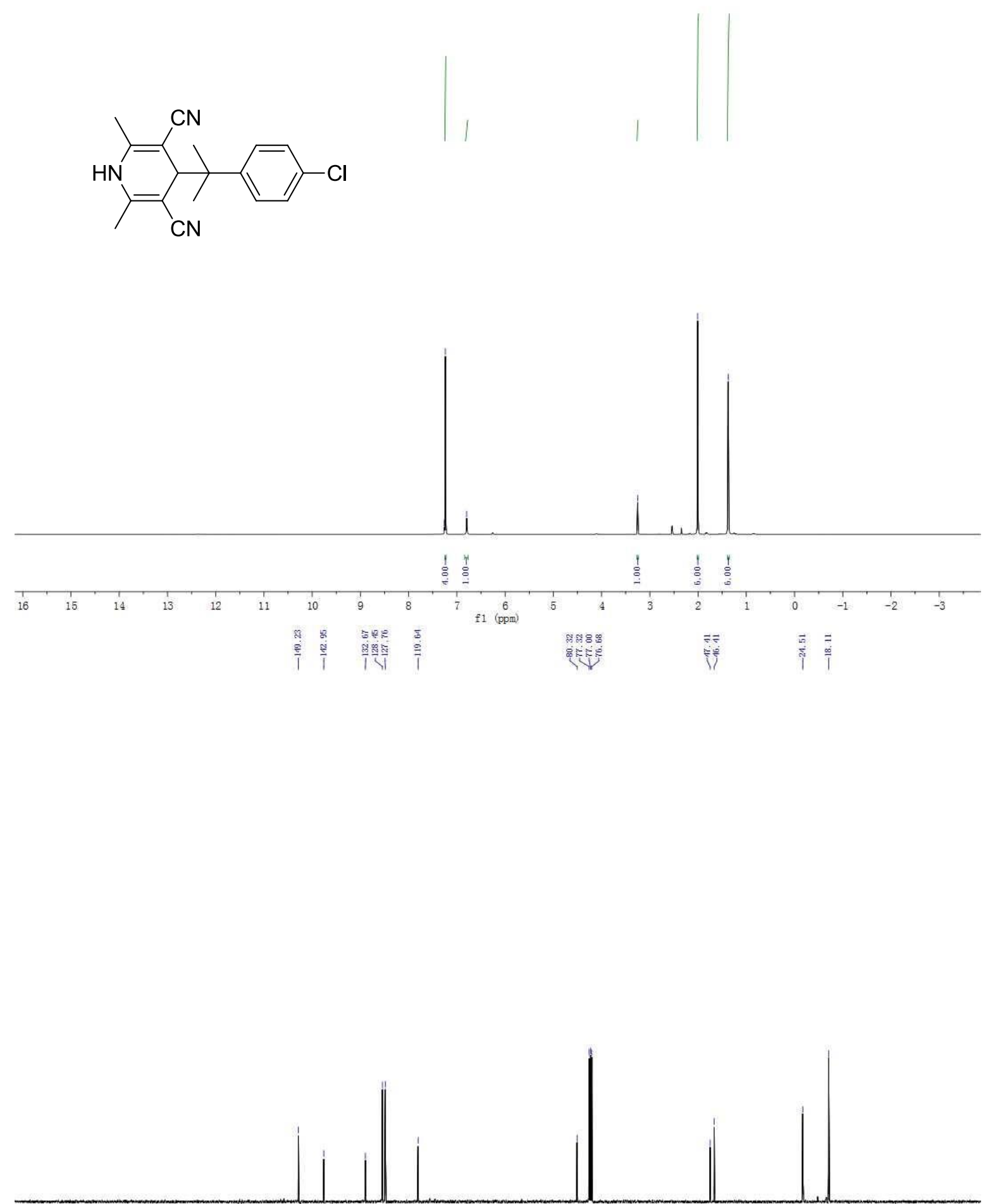
4-(2-(4-Bromophenyl)propan-2-yl)-2,6-dimethyl-1,4-dihydropyridine-3,5-dicarbonitrile(7d)<smiles>CC1=C(C#N)C(C(C)(C)c2ccc(Br)cc2)C(C#N)=C(C)N1</smiles>

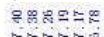

जाय?

î.
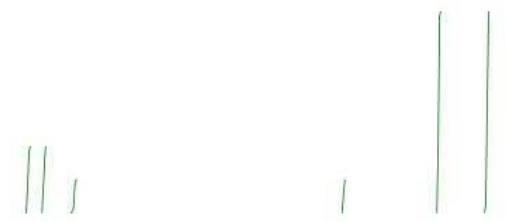
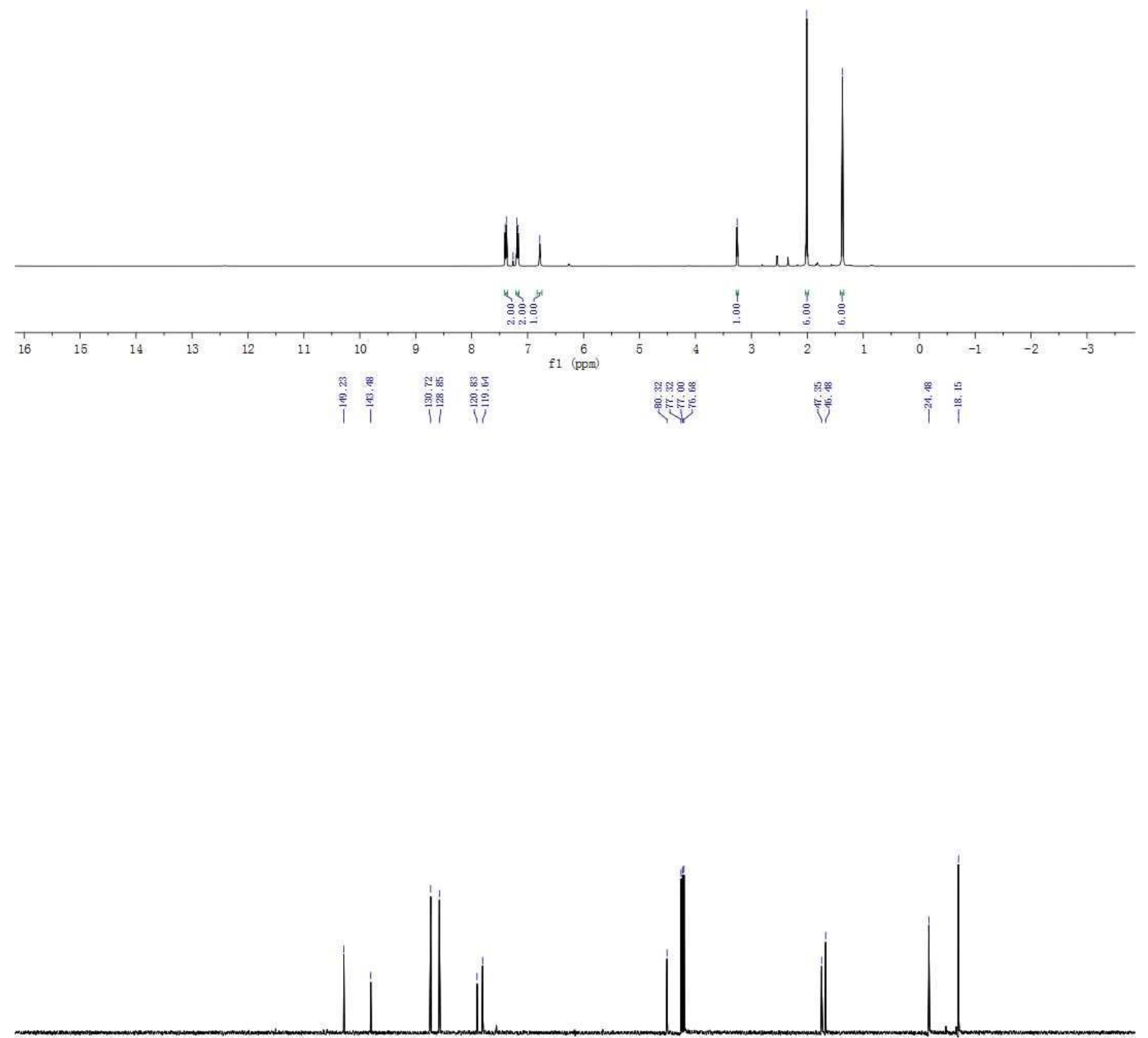
2,6-Dimethyl-4-(2-(4-(trifluoromethyl)phenyl)propan-2-yl)-1,4-dihydropyridine-3,5-dicarbonitril e(7e)<smiles>CC1=C(C#N)C(C(C)(C)c2ccc(C(F)(F)F)cc2)C(C#N)=C(C)N1</smiles><smiles>C1=CC=C1</smiles><smiles>C1CCCC1</smiles>
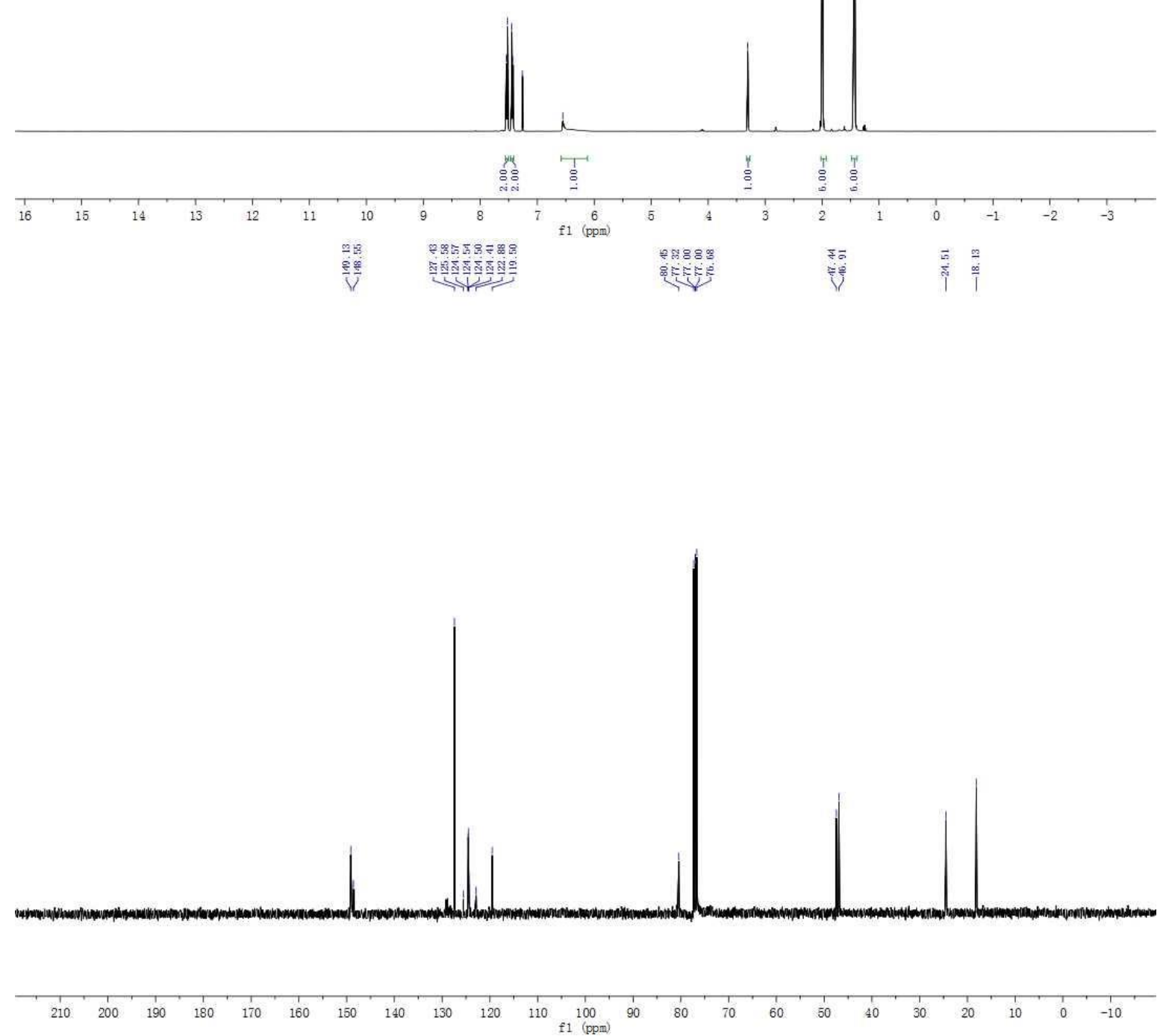
4-(1-(4-Chlorophenyl)cyclopentyl)-2,6-dimethyl-1,4-dihydropyridine-3,5-dicarbonitrile(7f)

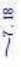
i
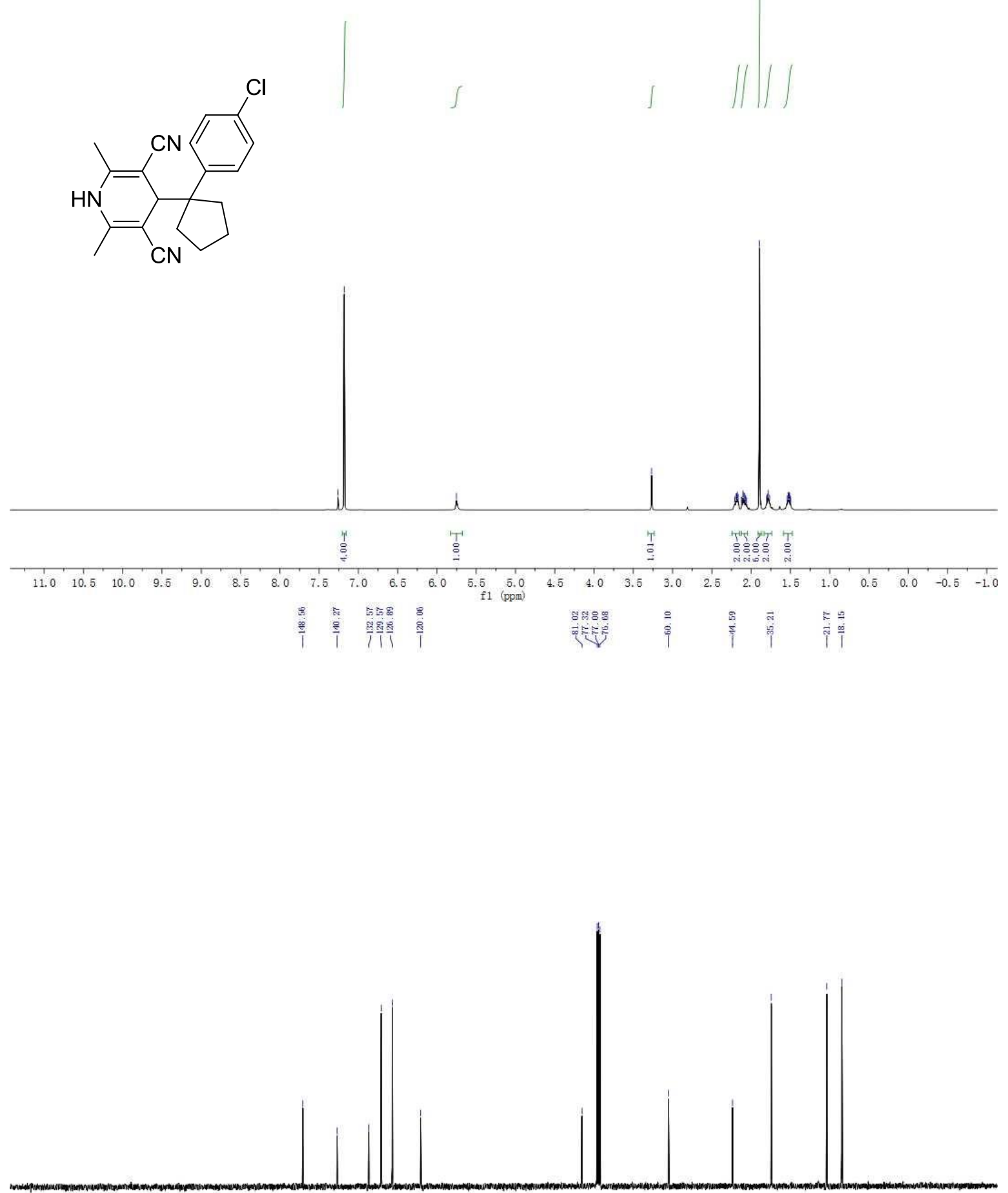
2,6-Dimethyl-4-(2-(thiophen-2-yl)propan-2-yl)-1,4-dihydropyridine-3,5-dicarbonitrile(7g)

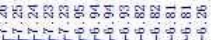
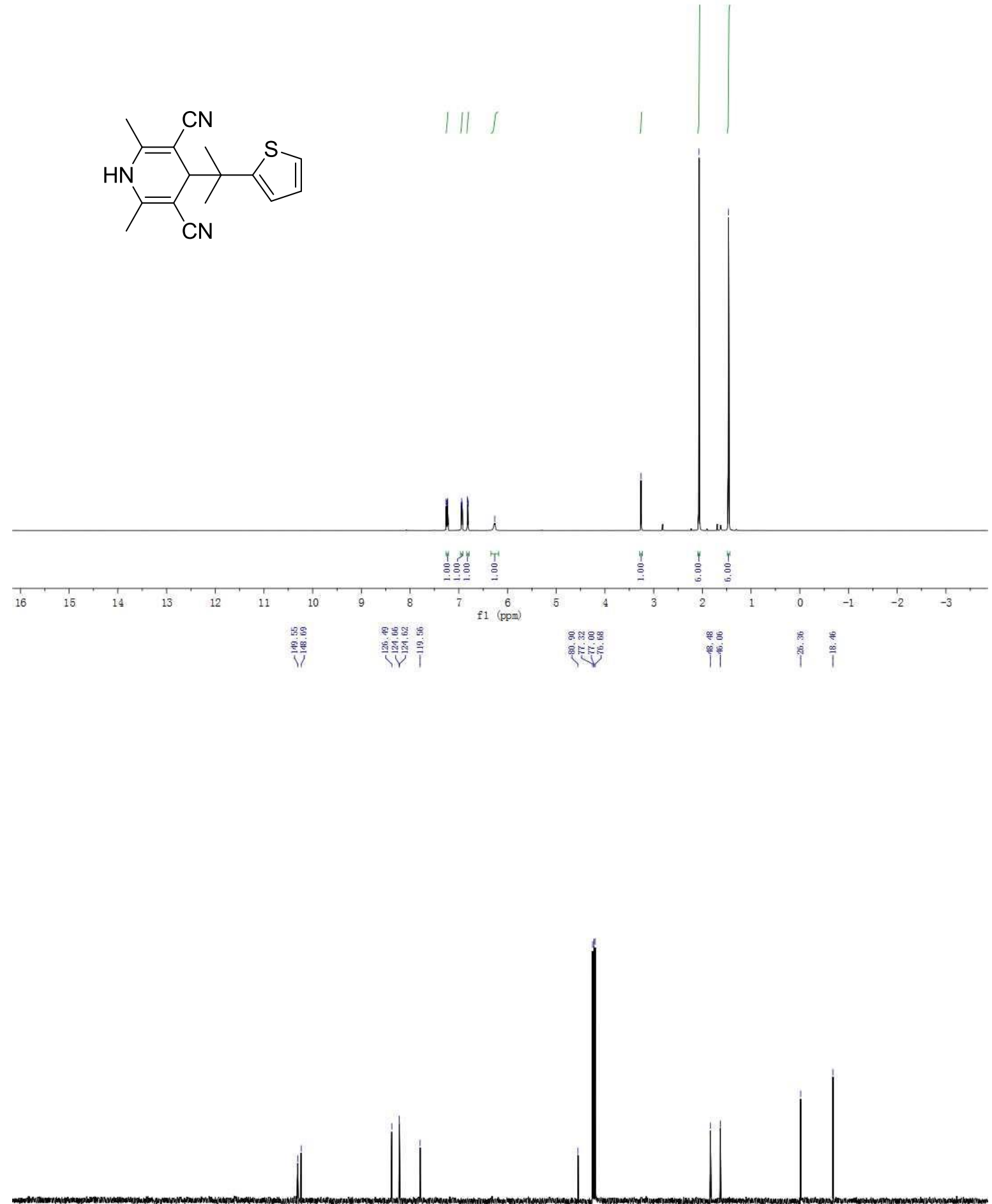
4-(2-(Benzofuran-2-yl)propan-2-yl)-2,6-dimethyl-1,4-dihydropyridine-3,5-dicarbonitrile(7h)

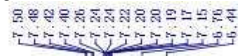<smiles>CC1=C(C#N)C(C(C)(C)c2cc3ccccc3o2)C(C#N)=C(C)N1</smiles>
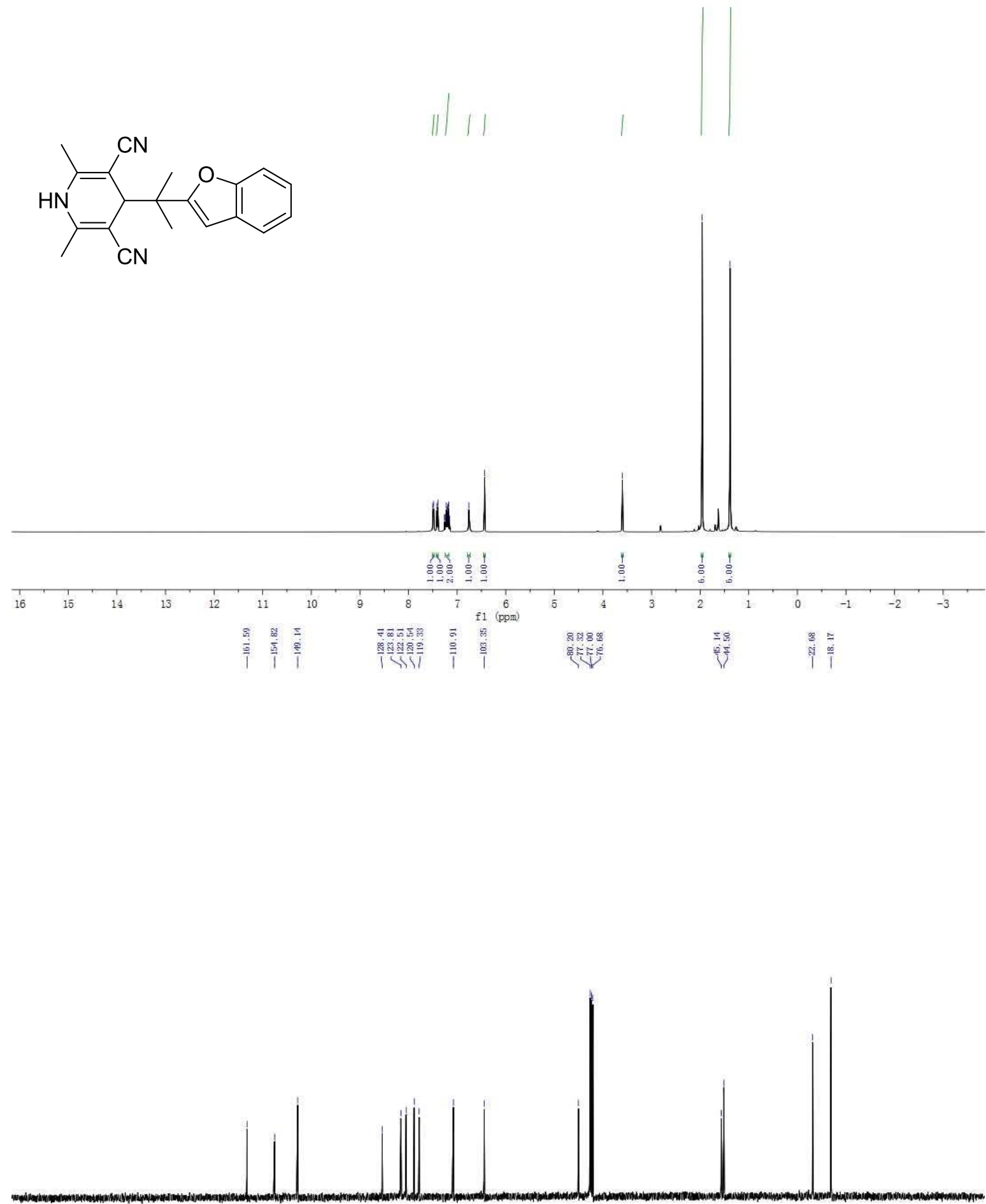
4-Benzhydryl-2,6-dimethyl-1,4-dihydropyridine-3,5-dicarbonitrile(7i)<smiles>CC1=C(C)C(C(c2ccccc2)c2ccccc2)C(C#N)=C(C)N1</smiles>
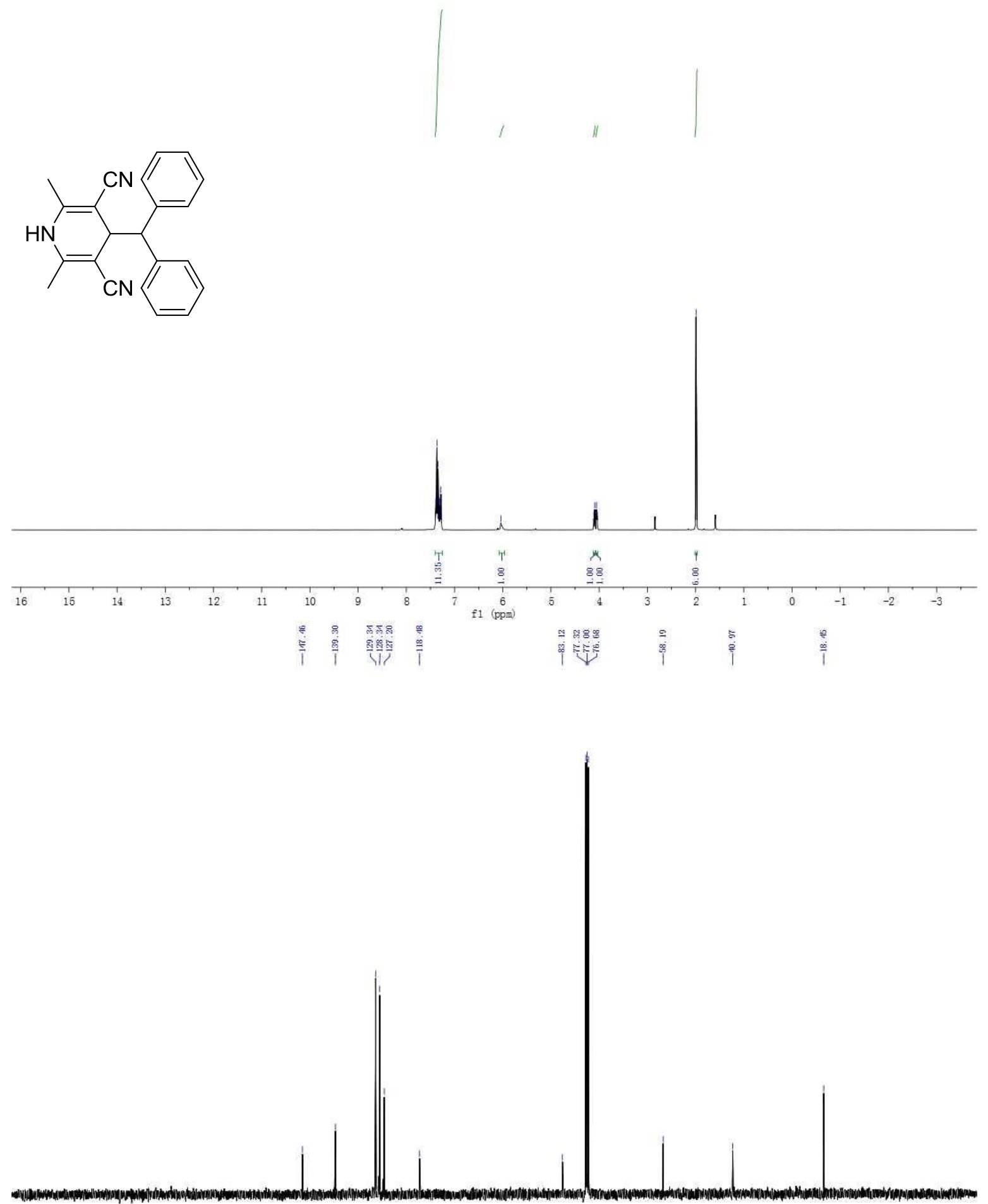

210 $180 \quad 17$ $160 \quad 150$ 130 20 f1 100 (ppm) 
2,6-dimethyl-4-(2-phenylbutan-2-yl)-1,4-dihydropyridine-3,5-dicarbonitrile (7j)

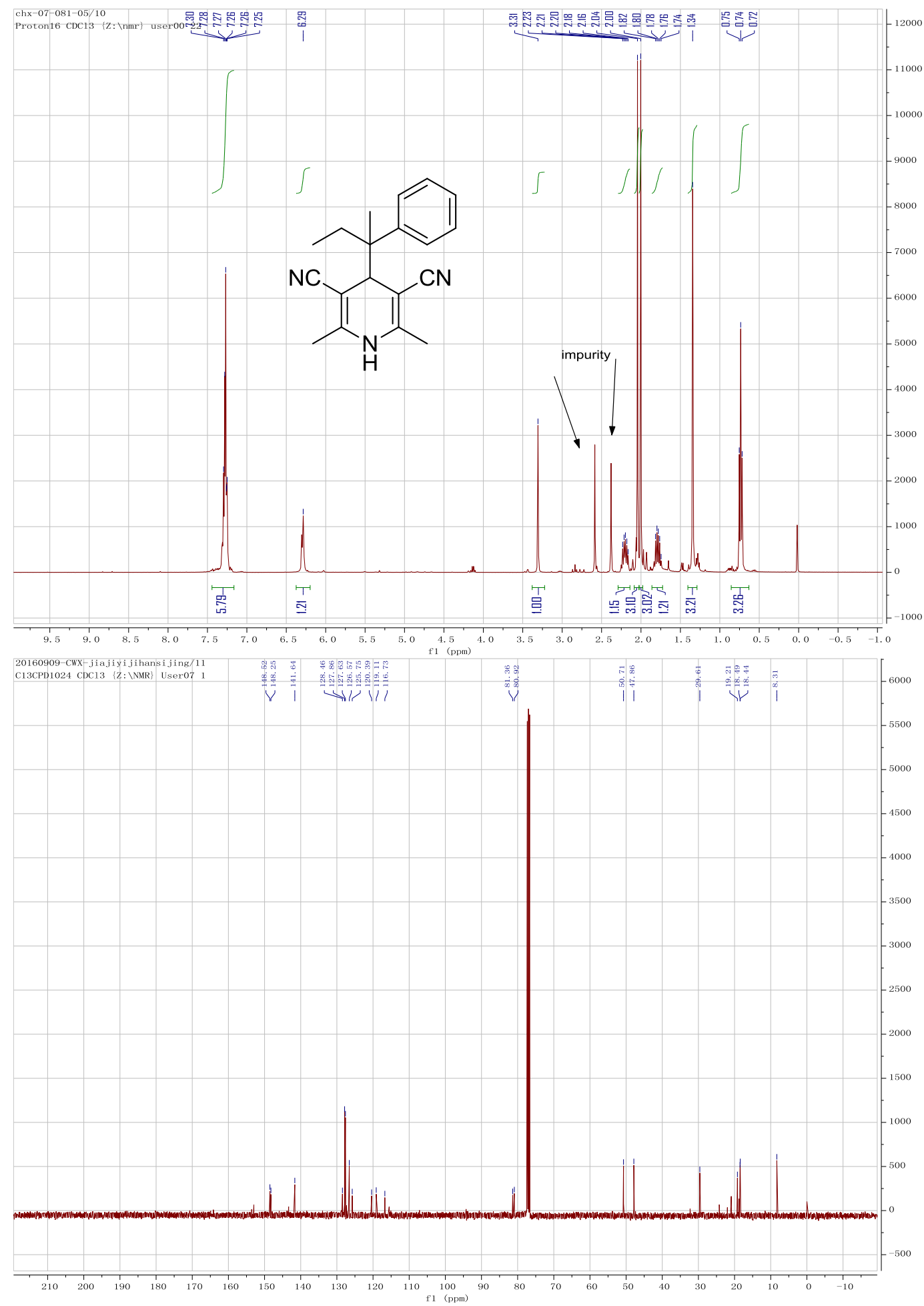


2,2,3-Trimethyl-1,3-diphenylbutan-1-one (8a)

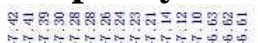
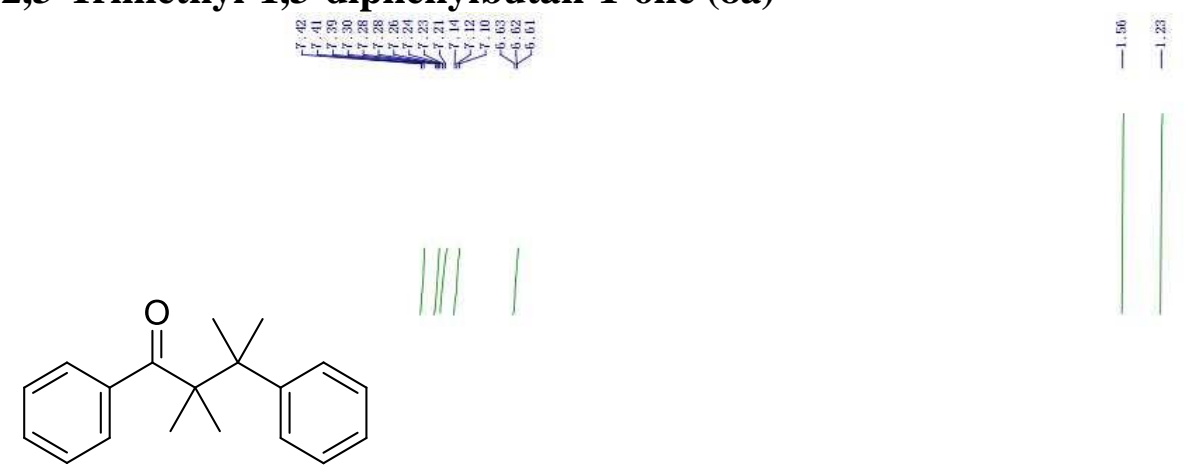

$\stackrel{8}{i}$
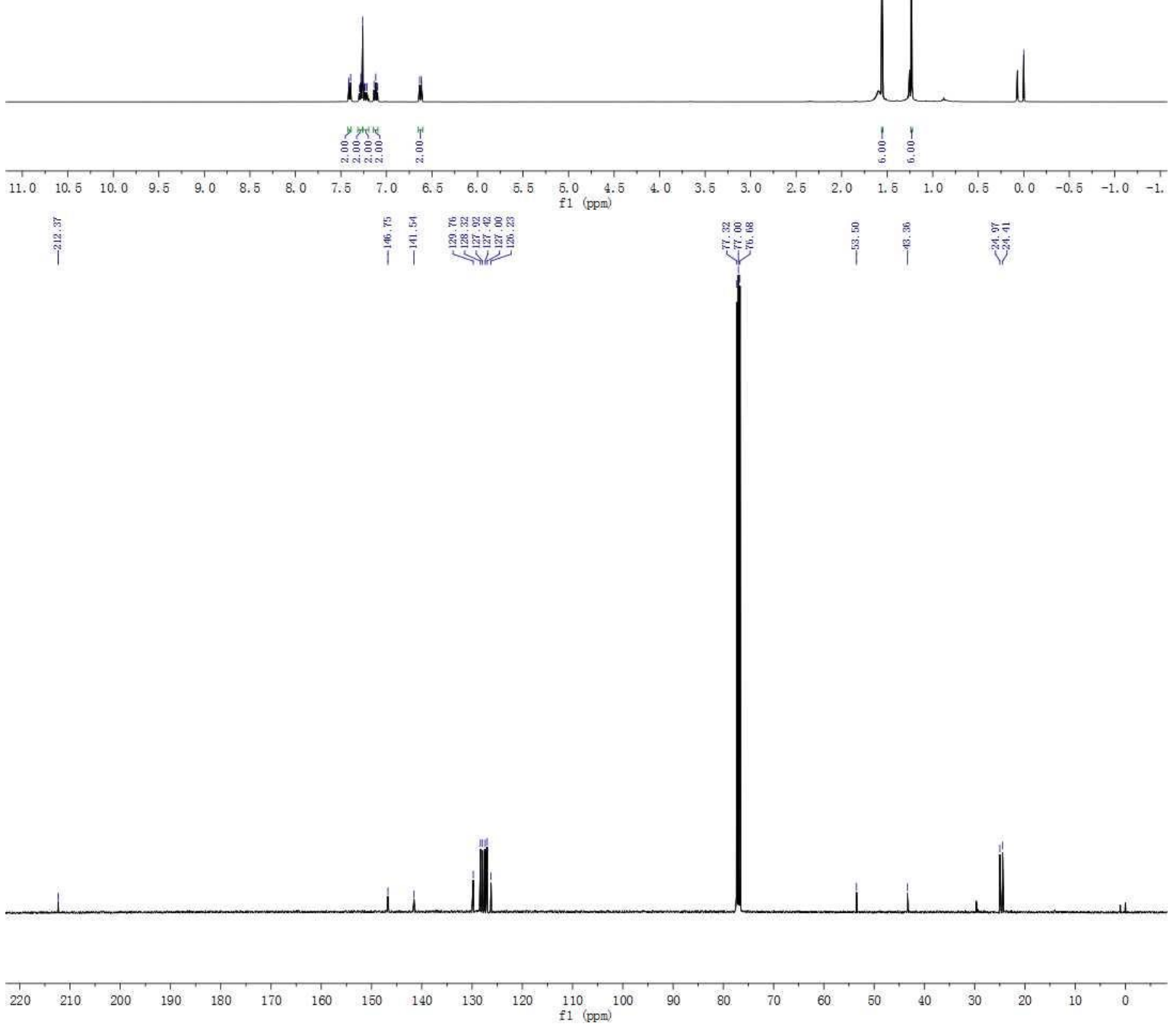

S102 


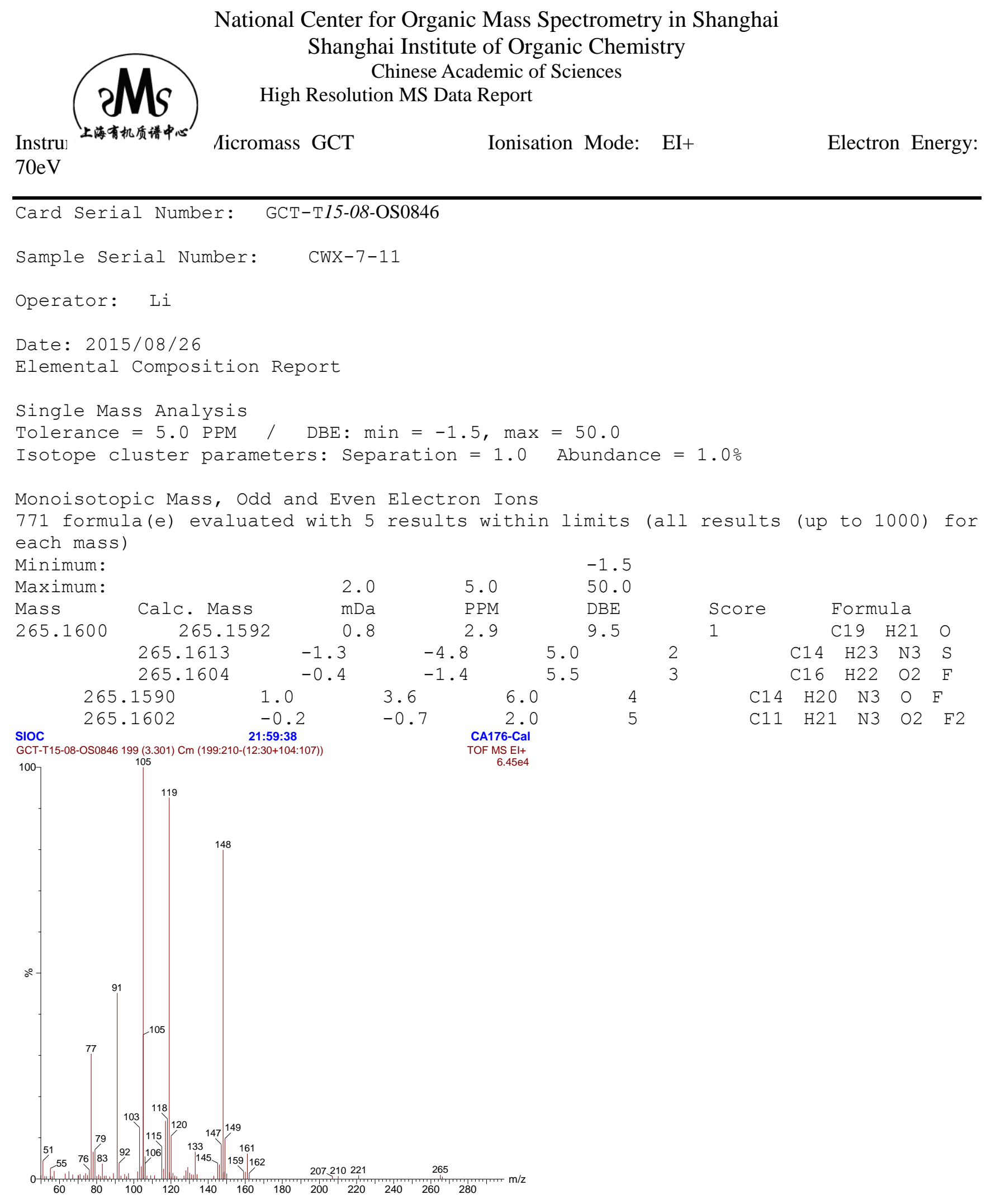


3-(4-Methoxyphenyl)-2,2,3-trimethyl-1-phenylbutan-1-one (8b)

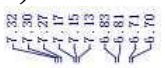
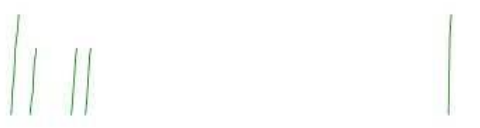

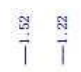

8
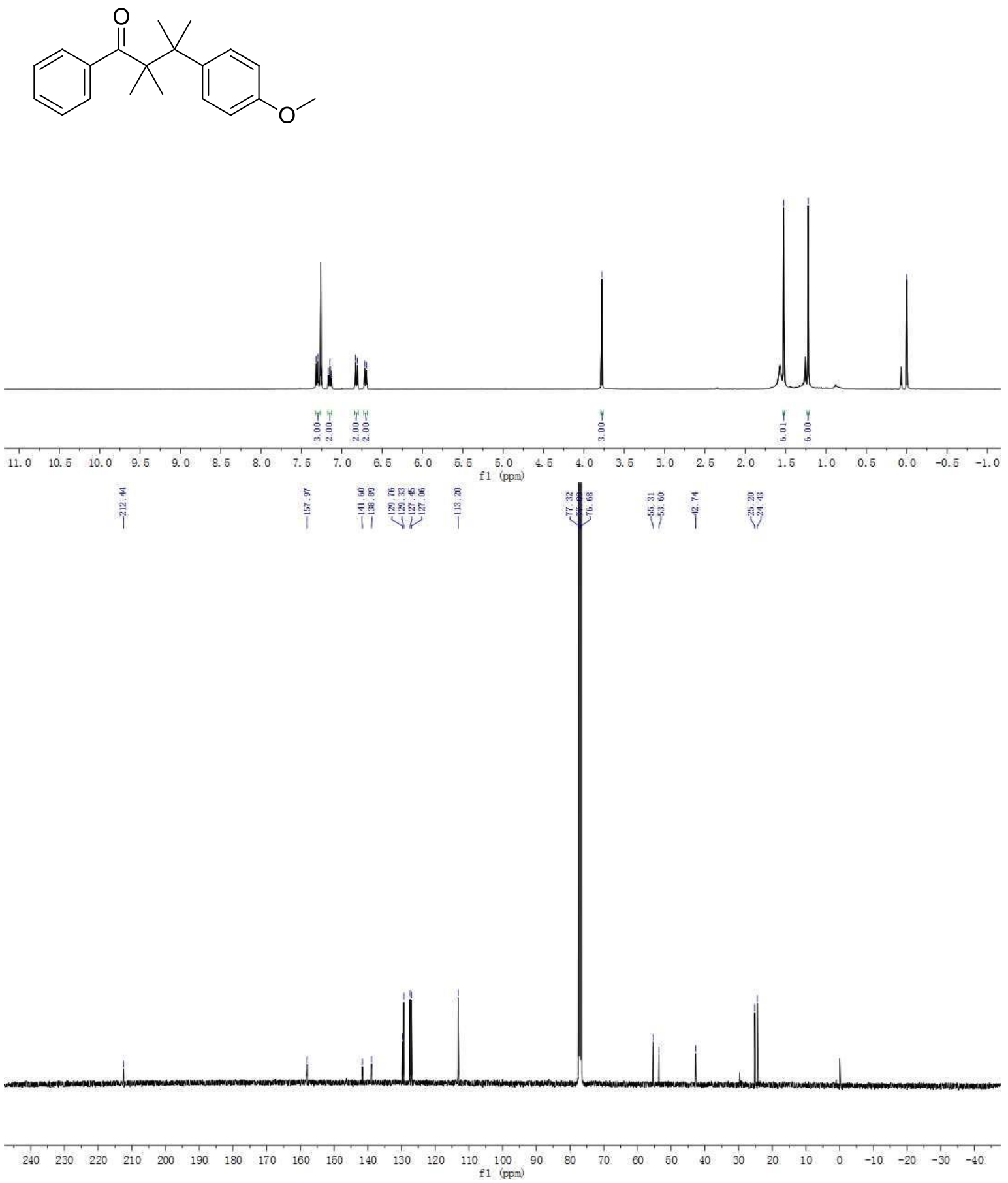

S104 
3-(4-Chlorophenyl)-2,2,3-trimethyl-1-phenylbutan-1-one (8c) 9.9.

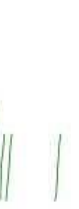<smiles>CC(C)(C(=O)c1ccccc1)C(C)(C)c1ccc(Cl)cc1</smiles>
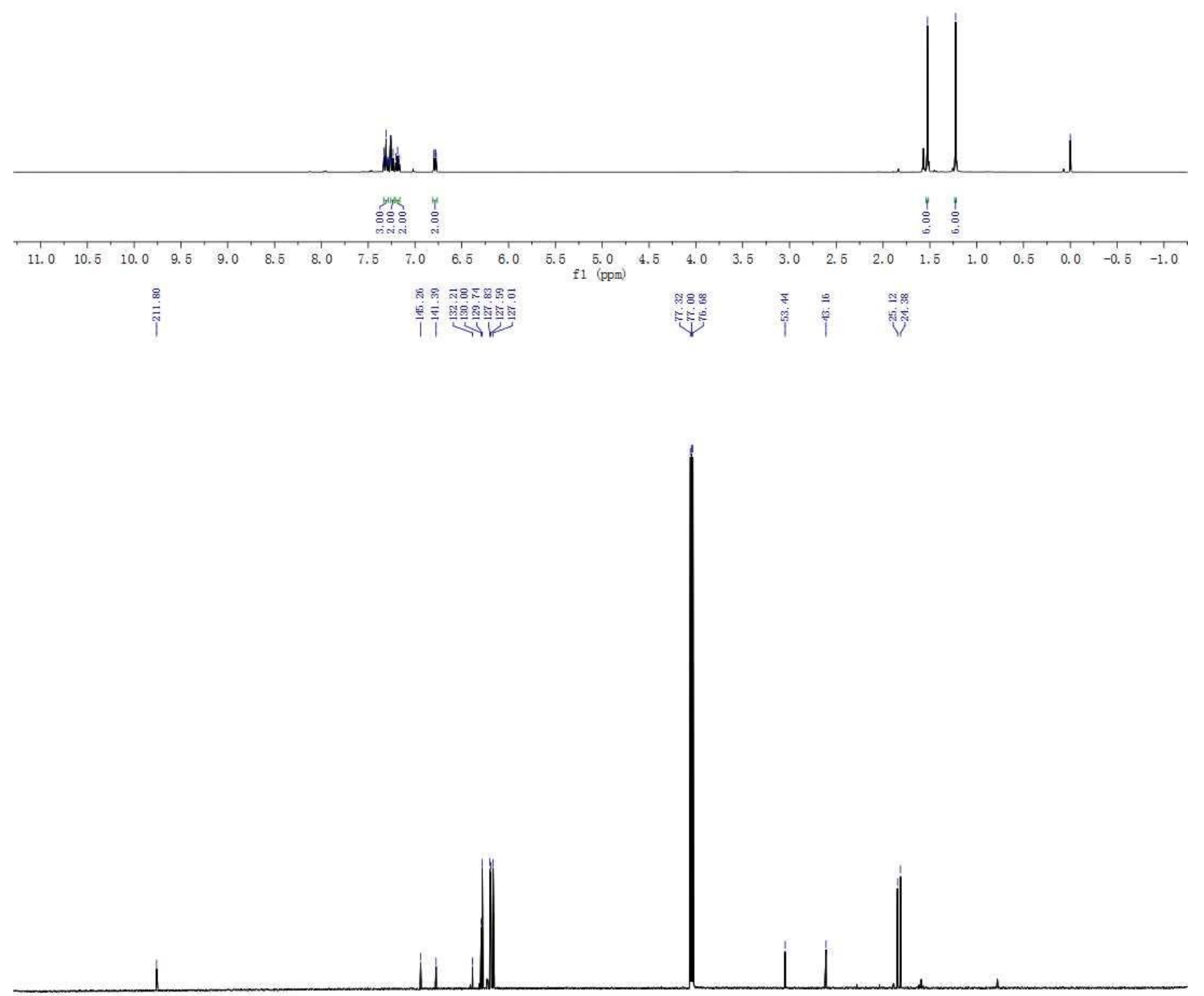

$\begin{array}{lllllllllllllllllllllllllllllllllllllllllll}240 & 230 & 220 & 210 & 200 & 190 & 180 & 170 & 160 & 150 & 140 & 130 & 120 & 110 & 100 & 90 & 80 & 70 & 60 & 50 & 40 & 30 & 20 & 10 & 0 & -10 & -20 & -30 & -40\end{array}$ 
3-(4-Bromophenyl)-2,2,3-trimethyl-1-phenylbutan-1-one (8d)

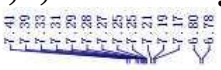
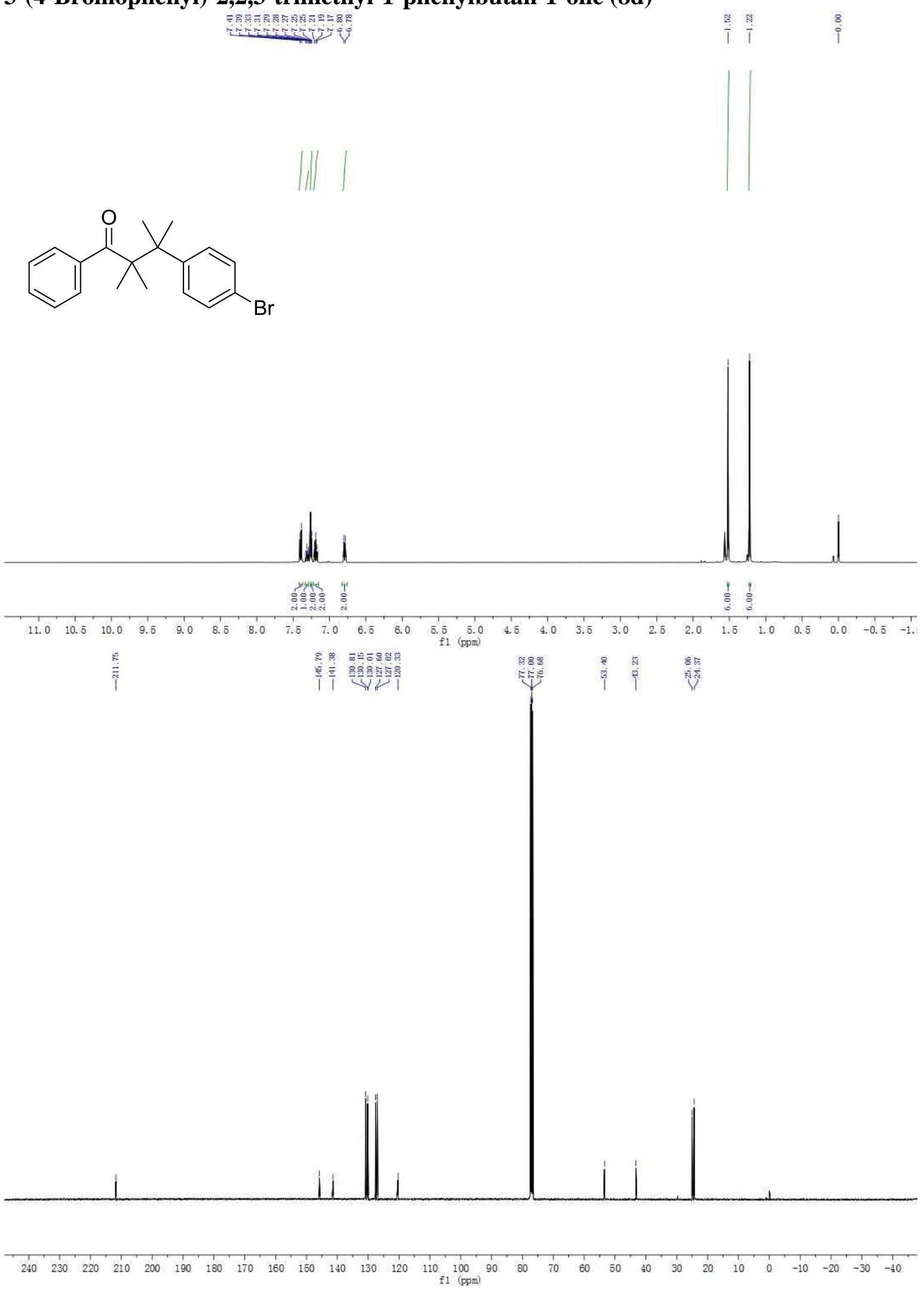

S106 
2,2,3-Trimethyl-1-phenyl-3-(4-(trifluoromethyl)phenyl)butan-1-one (8e)

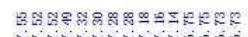

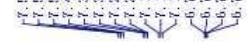
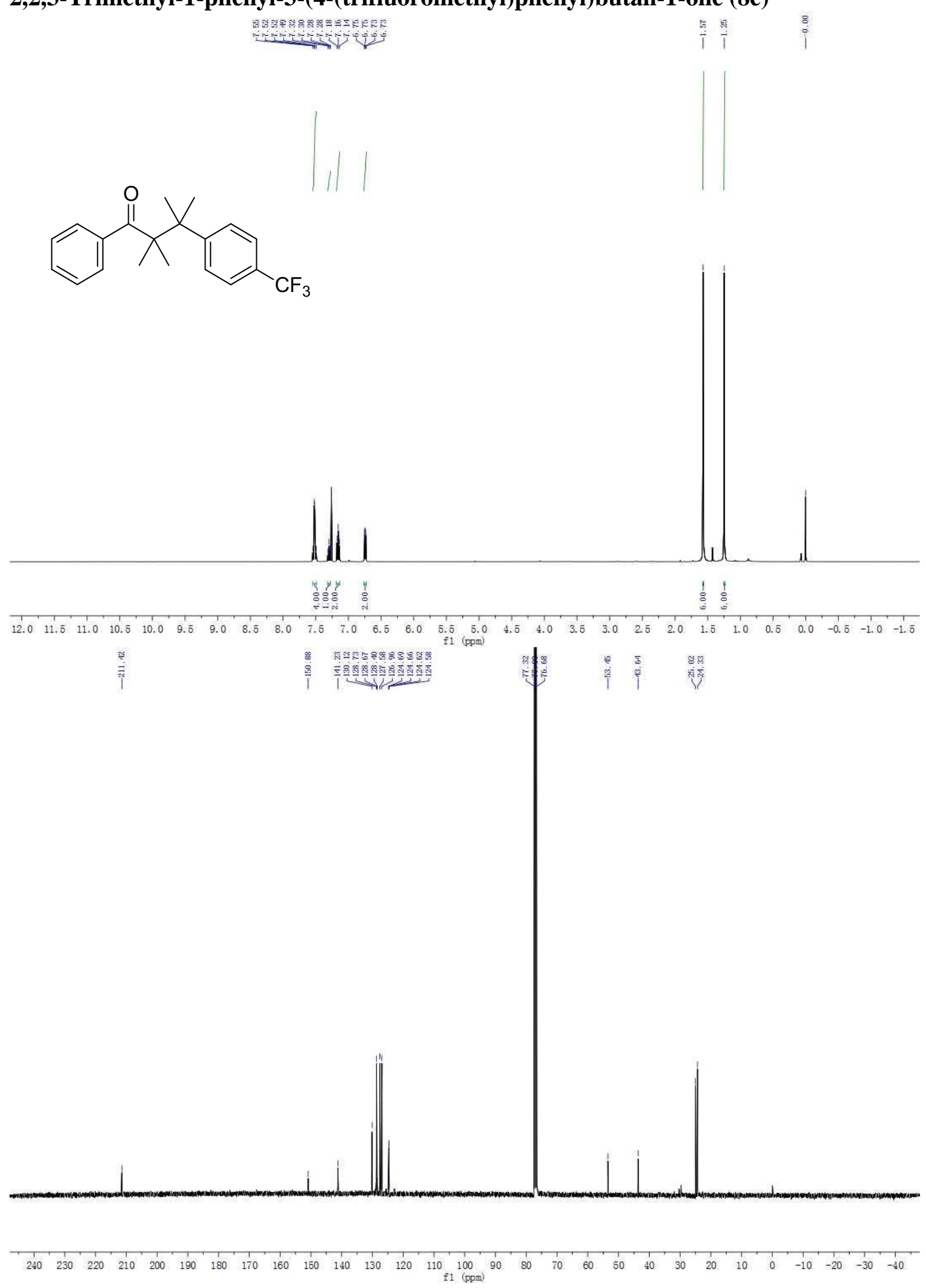

S107 
2-(1-(4-Chlorophenyl)cyclopentyl)-2-methyl-1-phenylpropan-1-one (8f)

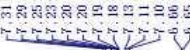

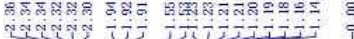
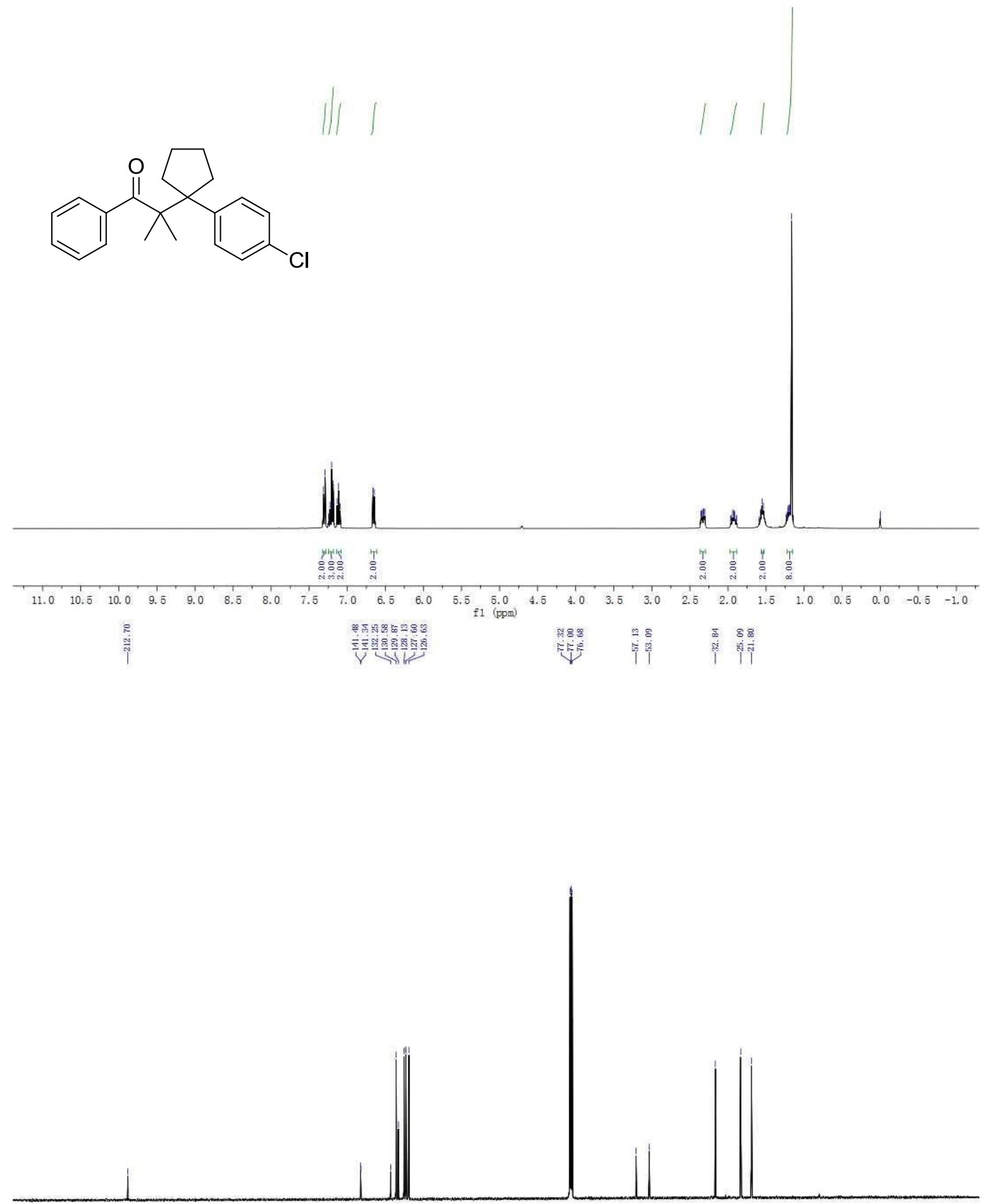

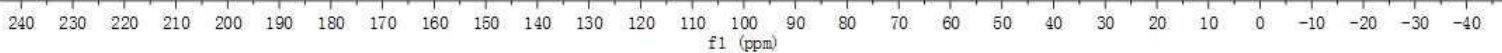


2,2,3-Trimethyl-1-phenyl-3-(thiophen-2-yl)butan-1-one ( $8 \mathrm{~g}$ )

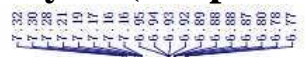
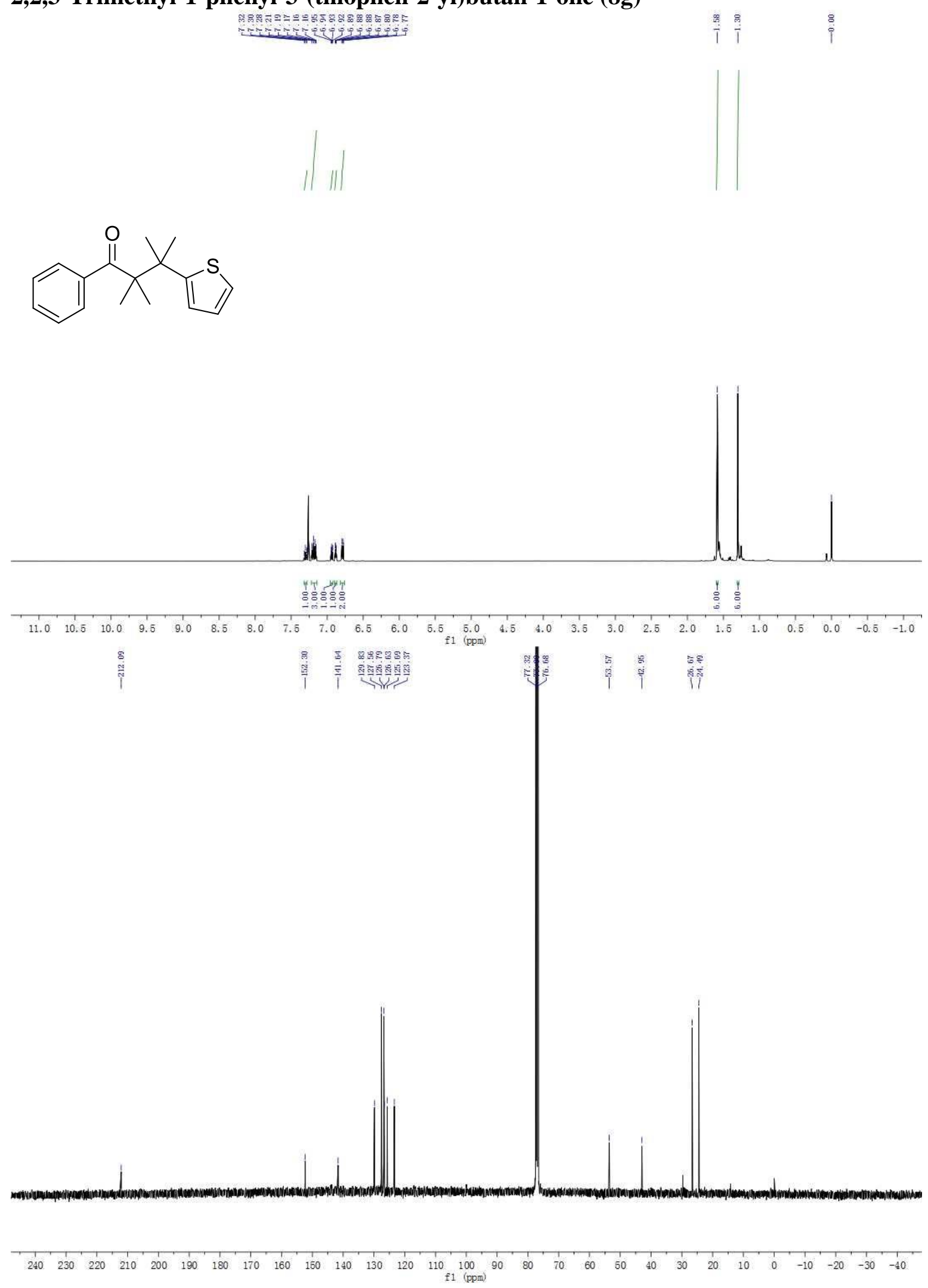

S109 
3-(Benzofuran-2-yl)-2,2,3-trimethyl-1-phenylbutan-1-one (8h)

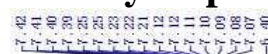

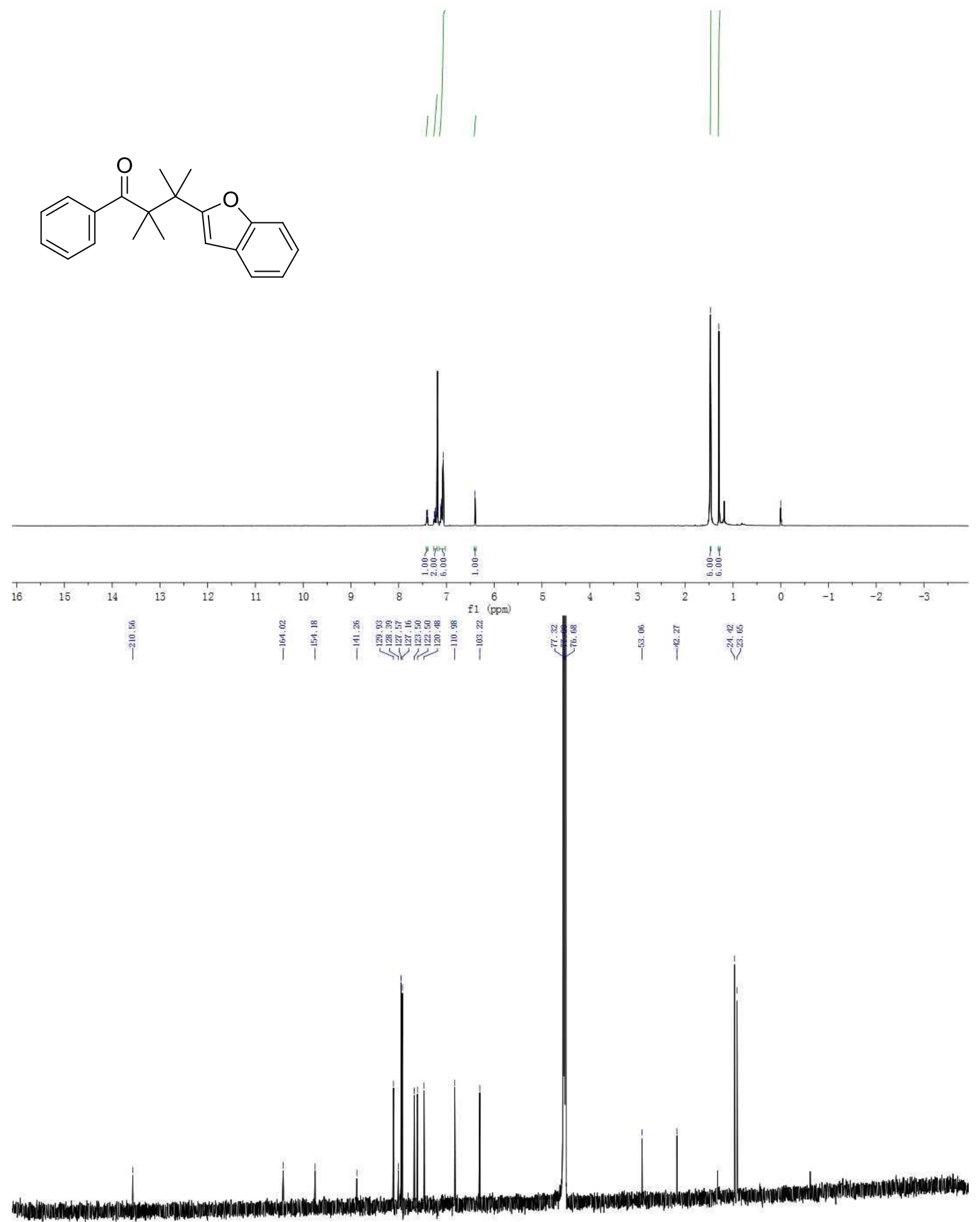

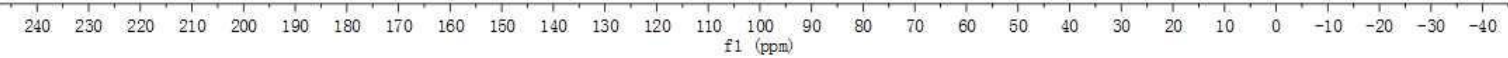


3-(4-Chlorophenyl)-2,2,3-trimethyl-1-(thiophen-3-yl)butan-1-one (8i)

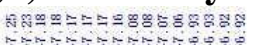

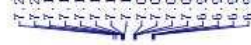

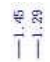

$\stackrel{0}{1}$
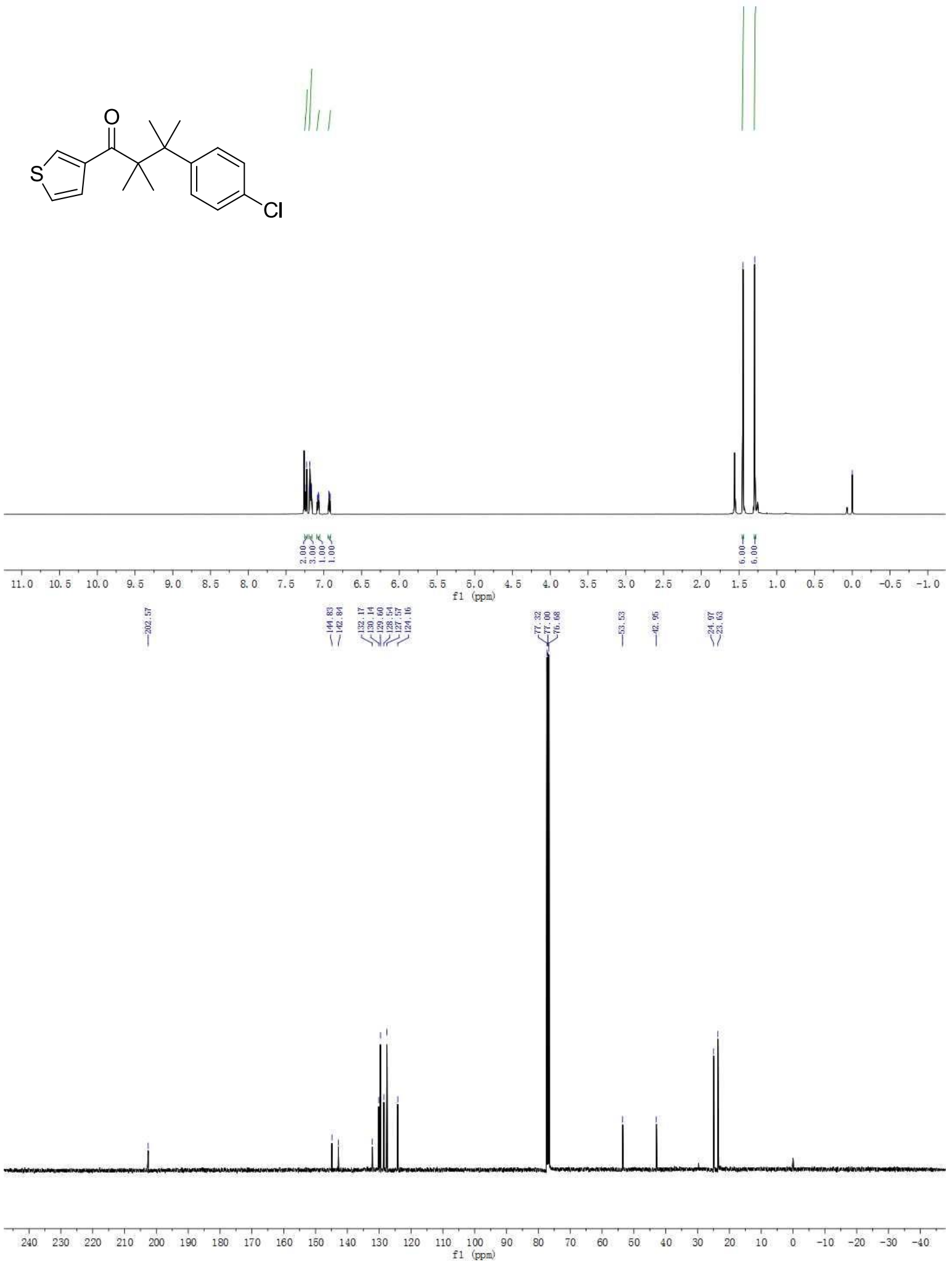

S111 
3-(4-Bromophenyl)-2,2,3-trimethyl-1-(thiophen-3-yl)butan-1-one (8j)

mе은

thistrisis

†

$\stackrel{8}{1}$
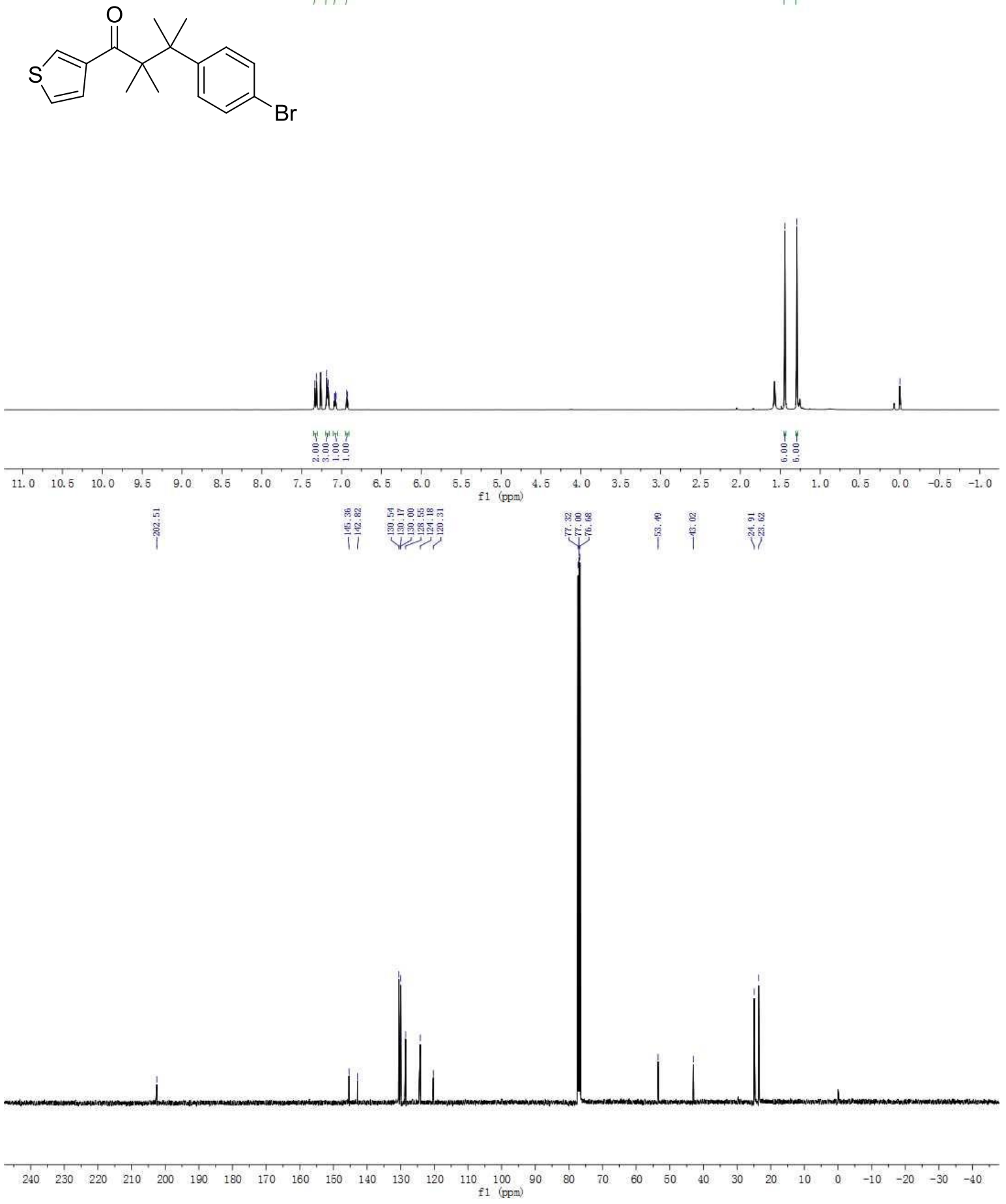

S112 
2,2,3-Trimethyl-1,3-di(thiophen-2-yl)butan-1-one (8k)

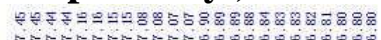

rer

$\stackrel{8}{11}$

11

| ||||
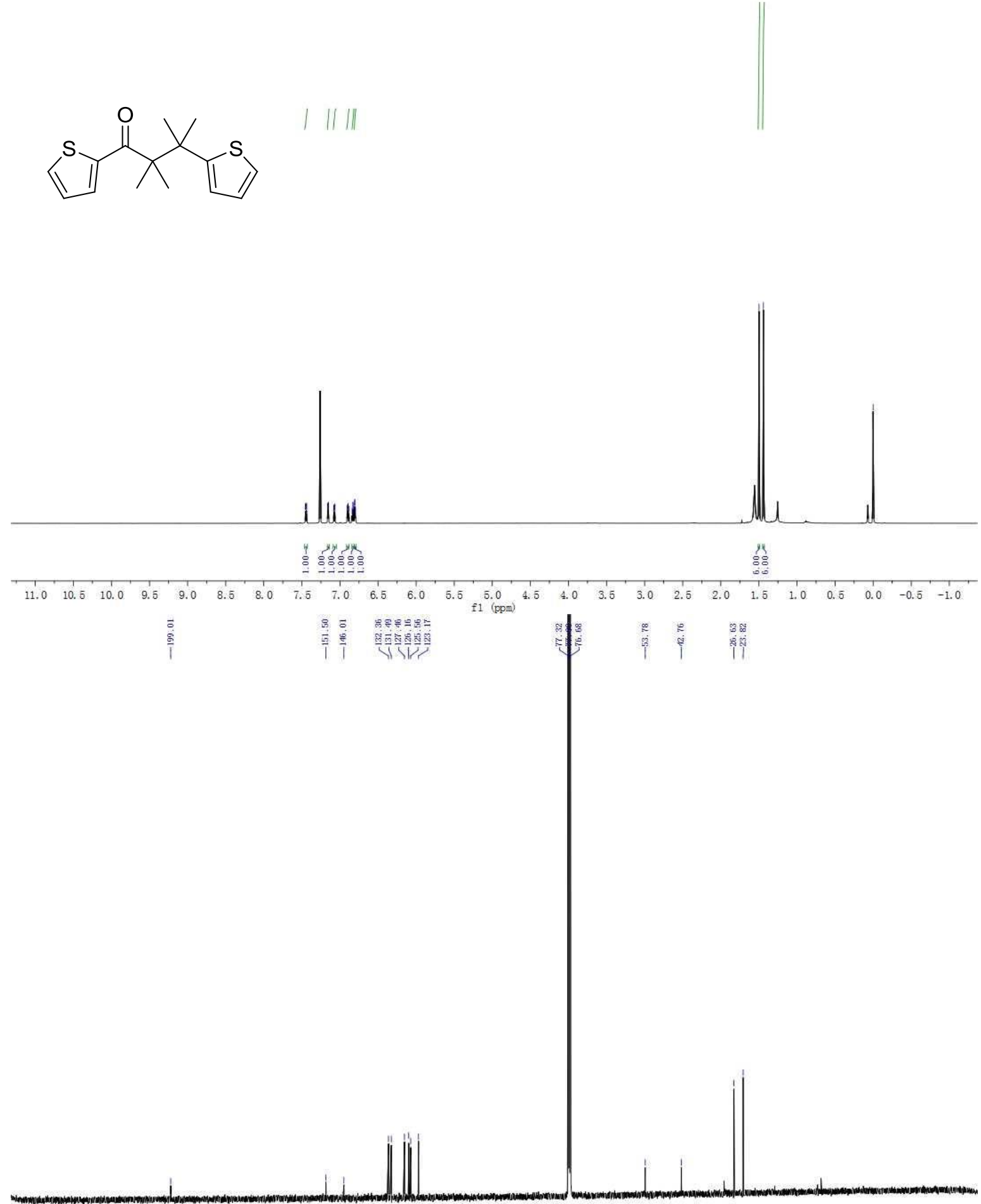

$\begin{array}{lllllllllllllllllllllllllllllllll}240 & 230 & 220 & 210 & 200 & 190 & 180 & 170 & 160 & 150 & 140 & 130 & 120 & 110 & 100 & 90 & 80 & 70 & 60 & 50 & 40 & 30 & 20 & 10 & 0 & -10 & -20 & -30 & -40\end{array}$ 
2,2,3-Trimethyl-3-(thiophen-2-yl)-1-(thiophen-3-yl)butan-1-one (81)

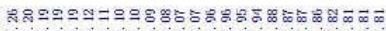

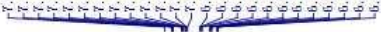<smiles>CC(C)(C(=O)c1ccsc1)C(C)(C)c1cccs1</smiles>

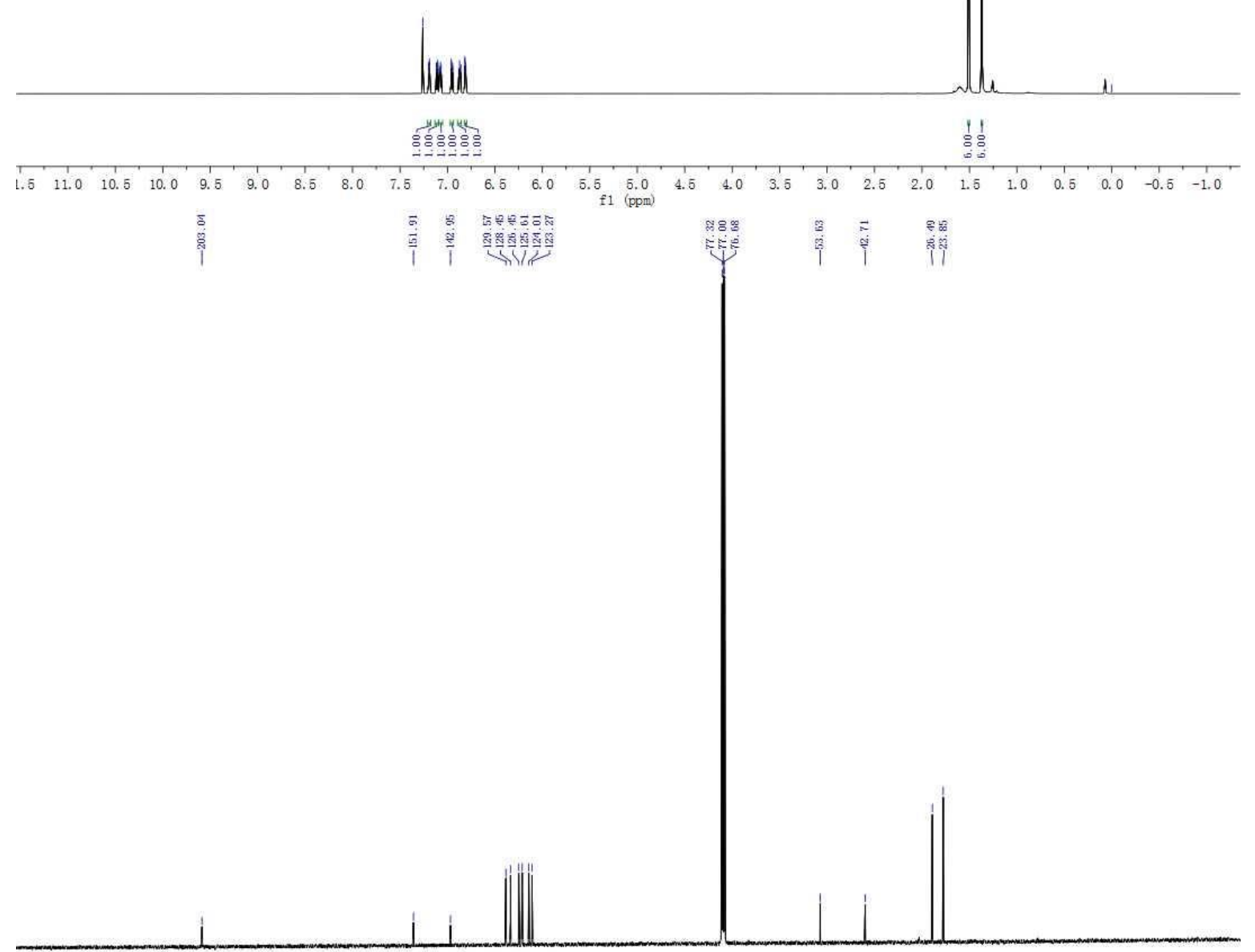

$\begin{array}{llllllllllllllllllllllllllllllll}240 & 230 & 220 & 210 & 200 & 190 & 180 & 170 & 160 & 150 & 140 & 130 & 120 & 110 & 100 & 90 & 80 & 70 & 60 & 50 & 40 & 30 & 20 & 10 & 0 & -10 & -20 & -30 & -40\end{array}$ 
2,2-Dimethyl-1,3,3-triphenylpropan-1-one (8m)

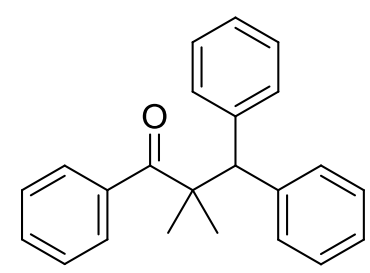

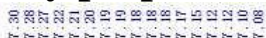

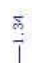

$\stackrel{8}{i}$
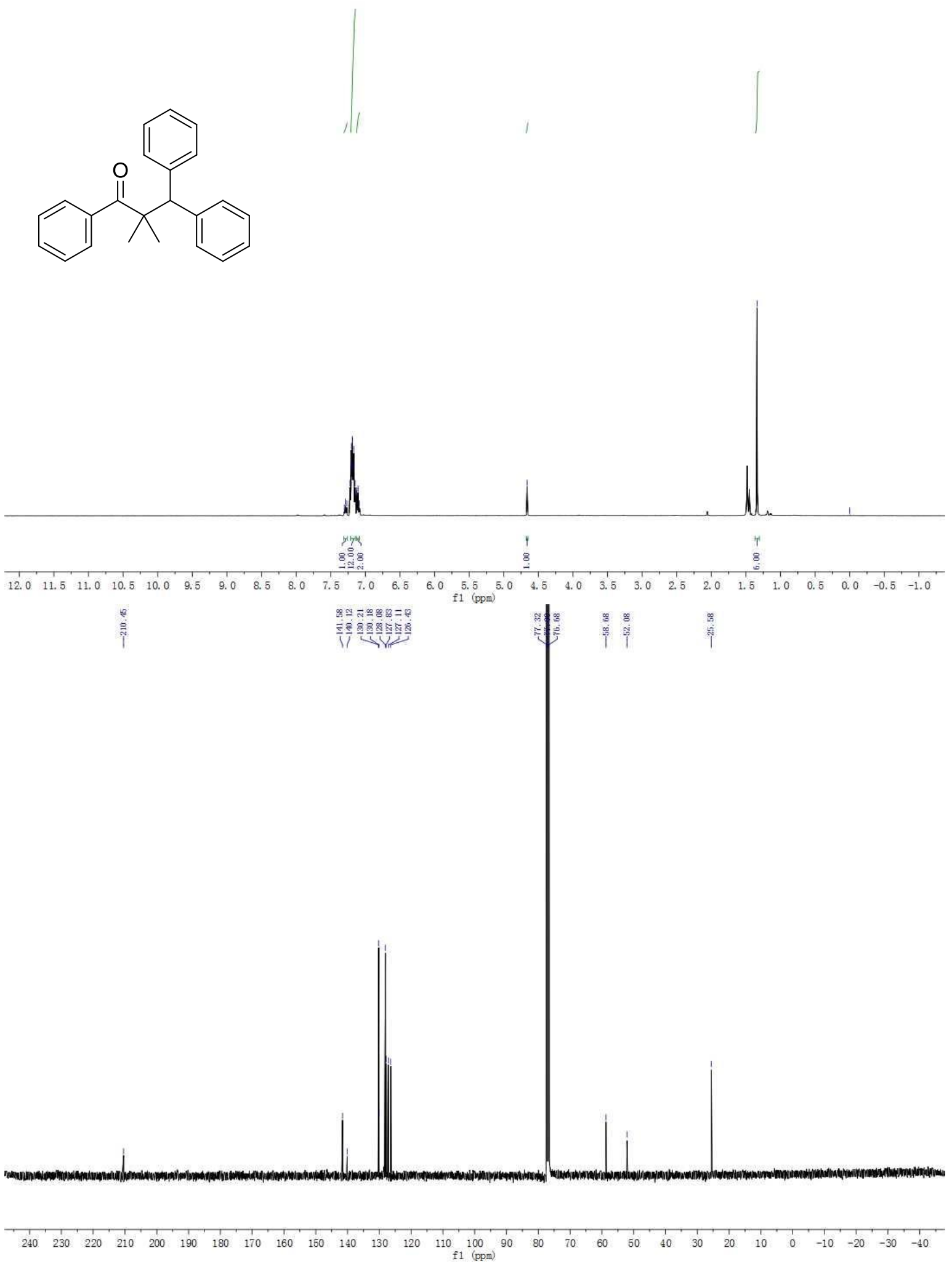

S115 
2,2,3-Trimethyl-1,3-diphenylpentan-1-one (8n)

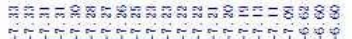

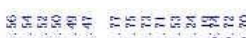

ن<smiles>CCC(C)(c1ccccc1)C(C)(C)C(=O)c1ccccc1</smiles>
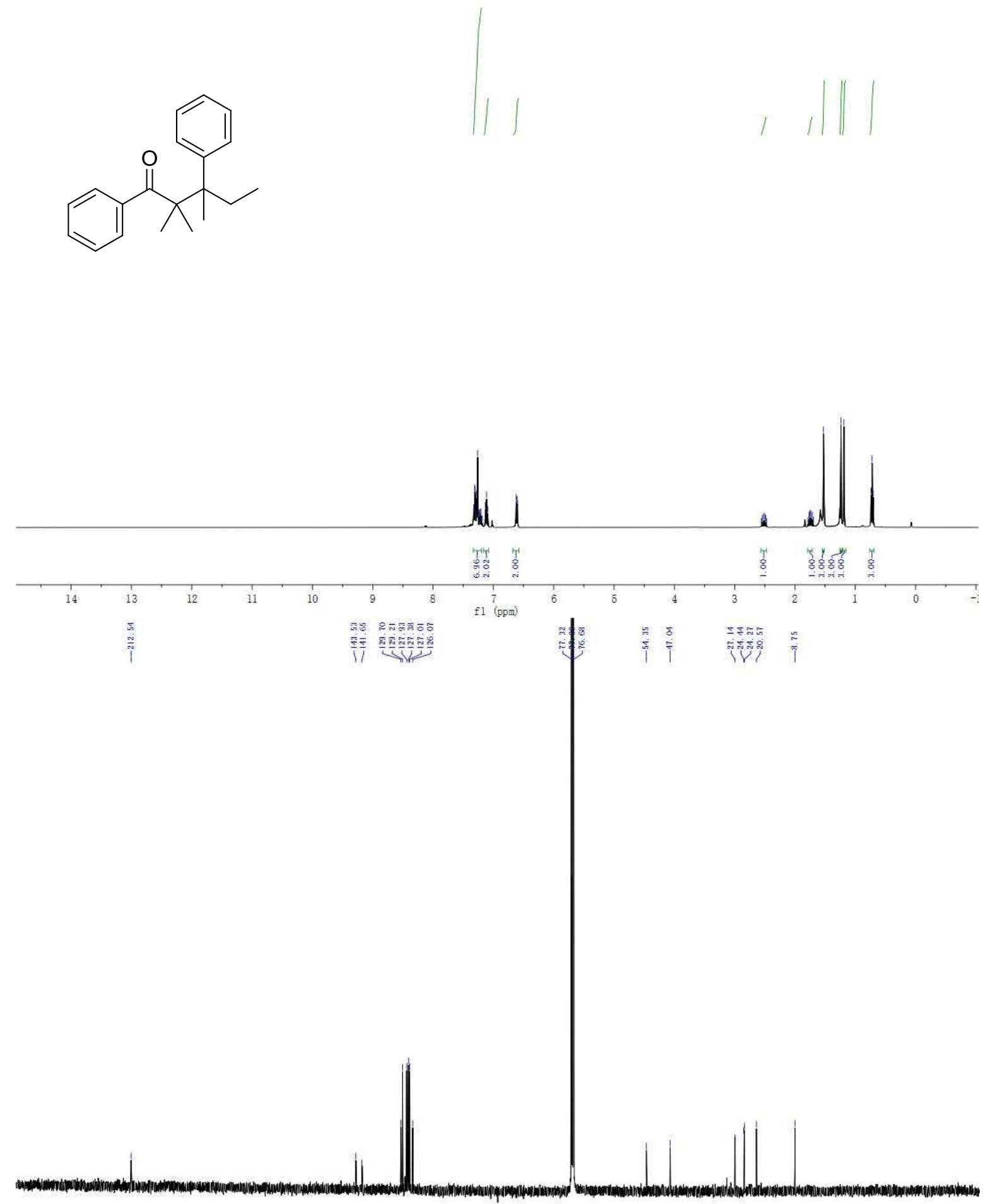

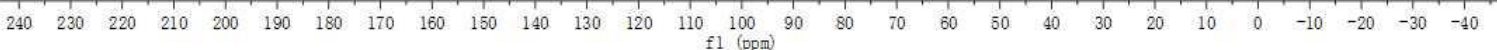

National Center for Organic Mass Spectrometry in Shanghai S116 
Shanghai Institute of Organic Chemistry

Chinese Academic of Sciences

High Resolution MS Data Report

Instrument: Waters Micromass GCT Premier

Ionisation Mode: EI+

Electron Energy: $70 \mathrm{eV}$

Card Serial Number: GCT-P-T16-09-0S1315

Sample Serial Number: NJU-CWX-8N

Operator: Li

Date: 2016/09/06

Elemental Composition Report

Single Mass Analysis

Tolerance $=5.0 \mathrm{PPM}$

Element prediction: off

$\mathrm{DBE}: \min =-1.5, \max =50.0$

Monoisotopic Mass, Odd and Even Electron Ions

386 formula(e) evaluated with 3 results within limits (all results (up to 1000) for each mass)

Elements Used:

$\mathrm{C}: 0-60 \quad \mathrm{H}: 0-80 \quad \mathrm{~N}: 0-2 \quad \mathrm{O}: 0-4 \quad \mathrm{~F}: 0-3 \quad \mathrm{~S}: 0-1$

Minimum:

Maximum:

Mass

$-1.5$

281.1910

Calc. Mass

2.0

5.0

50.0

281.1905

281.1917

281.1899

0.5

$\mathrm{mDa} \quad \mathrm{PPM}$

DBE

$$
-0.7
$$

1.1

2016-A-0681-NJU

$\begin{array}{llr}\text { GCT-P-T16-0681 } 823(14.127) & \mathrm{Cm}(821: 824-(814: 819+830: 838)) & \text { TOF MS El+ } \\ 100 & 91 & 148\end{array}$

100

$-2.5-4.5$

$-0.5$

8.5

4.5

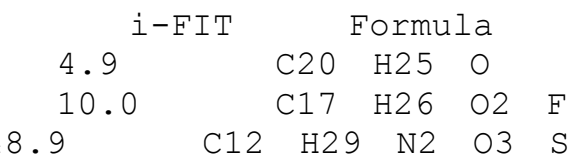

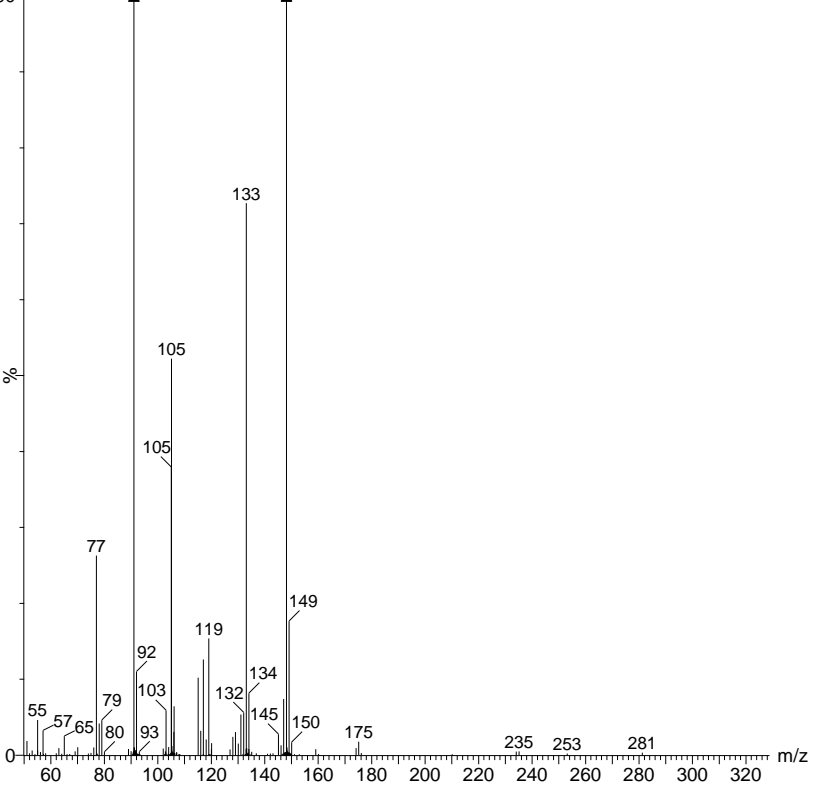




$$
\frac{m}{1}
$$


National Center for Organic Mass Spectrometry in Shanghai

Shanghai Institute of Organic Chemistry

Chinese Academic of Sciences

High Resolution MS Data Report

Instrument: Waters Micromass GCT Premier

Ionisation Mode: EI+

Electron

Energy: $70 \mathrm{eV}$

Card Serial Number: GCT-P-T16-09-0S1316

Sample Serial Number: NJU-CWX-80

Operator: Li

Date: $2016 / 09 / 06$

Elemental Composition Report

Single Mass Analysis

Tolerance $=5.0 \mathrm{PPM} / \mathrm{DBE}: \min =-1.5, \max =50.0$

Element prediction: Off

Monoisotopic Mass, Odd and Even Electron Ions

223 formula(e) evaluated with 2 results within limits (all results (up to 1000) for each mass)

Elements Used:

$\mathrm{C}: 0-60 \quad \mathrm{H}: 0-80 \quad \mathrm{~N}: 0-2 \quad \mathrm{O}: 0-4 \quad \mathrm{~S}: 0-1 \quad \mathrm{Cl}: 0-1$

Minimum:

5.0

$-1.5$

Maximum:

Mass

Calc. Mass

2.0

PPM

50.0

309.1614

309.1621

$\mathrm{mDa}$

$-0.7$

$-2.3$

$\mathrm{DBE}$

5.5

i-FIT

Formula

O2 Cl

309.1603

1.1

3.6

10.5

5546258.5

C1

H21 N2

C18 H26
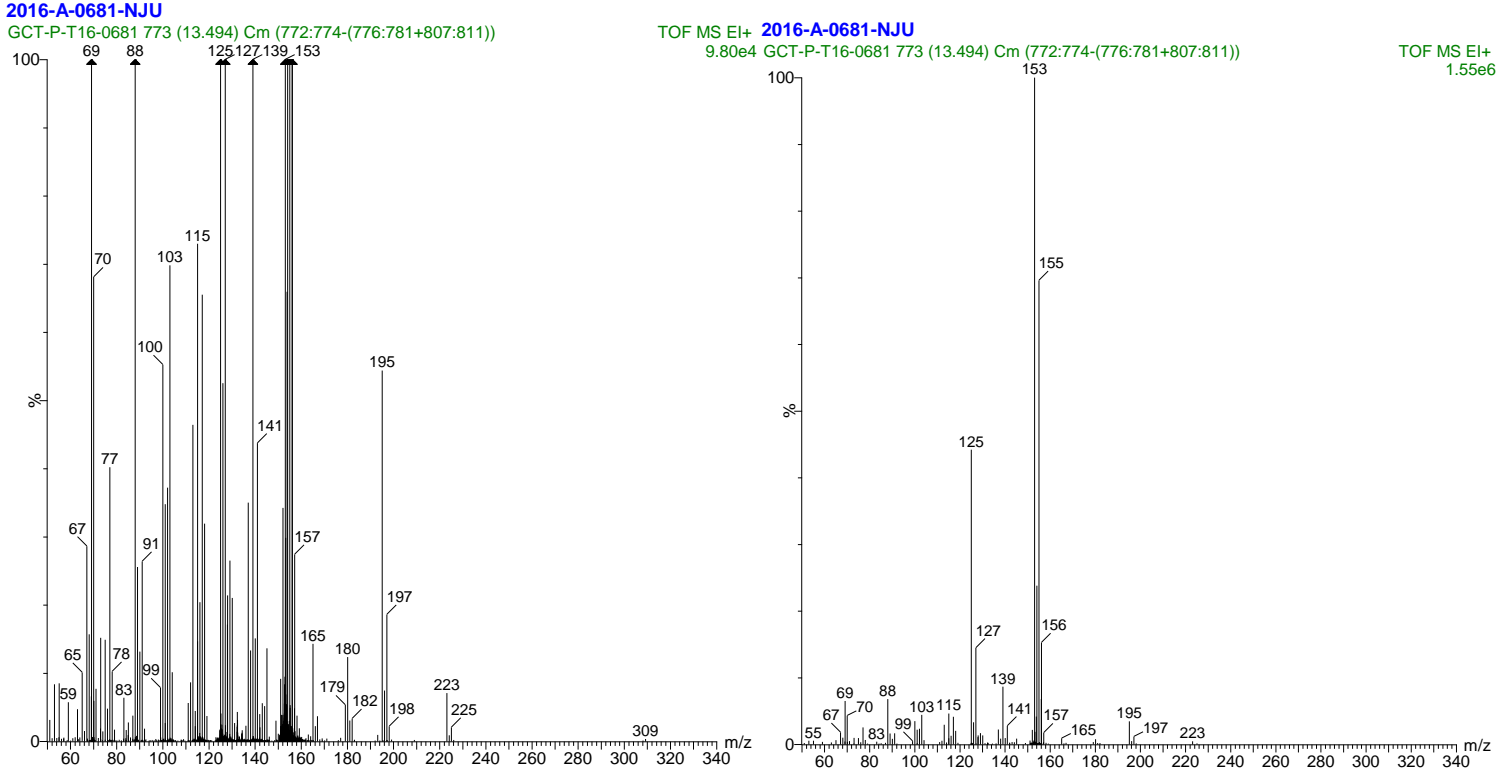
2,2,3-trimethyl-3-phenylbutanoic acid (8p)

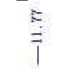

$+$

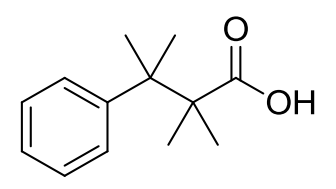

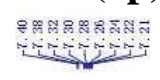

$\|$ $\stackrel{8}{i}=$

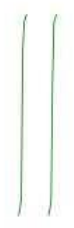

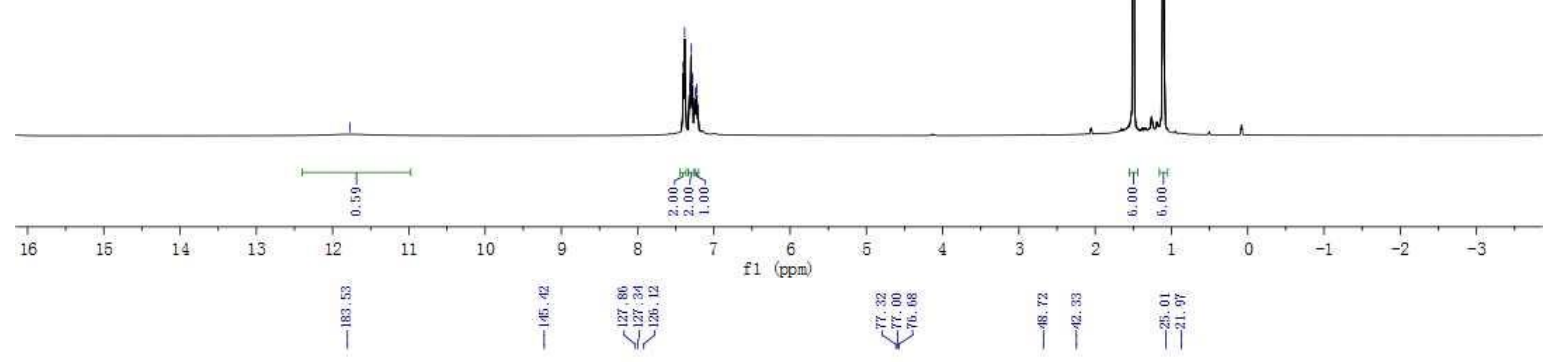
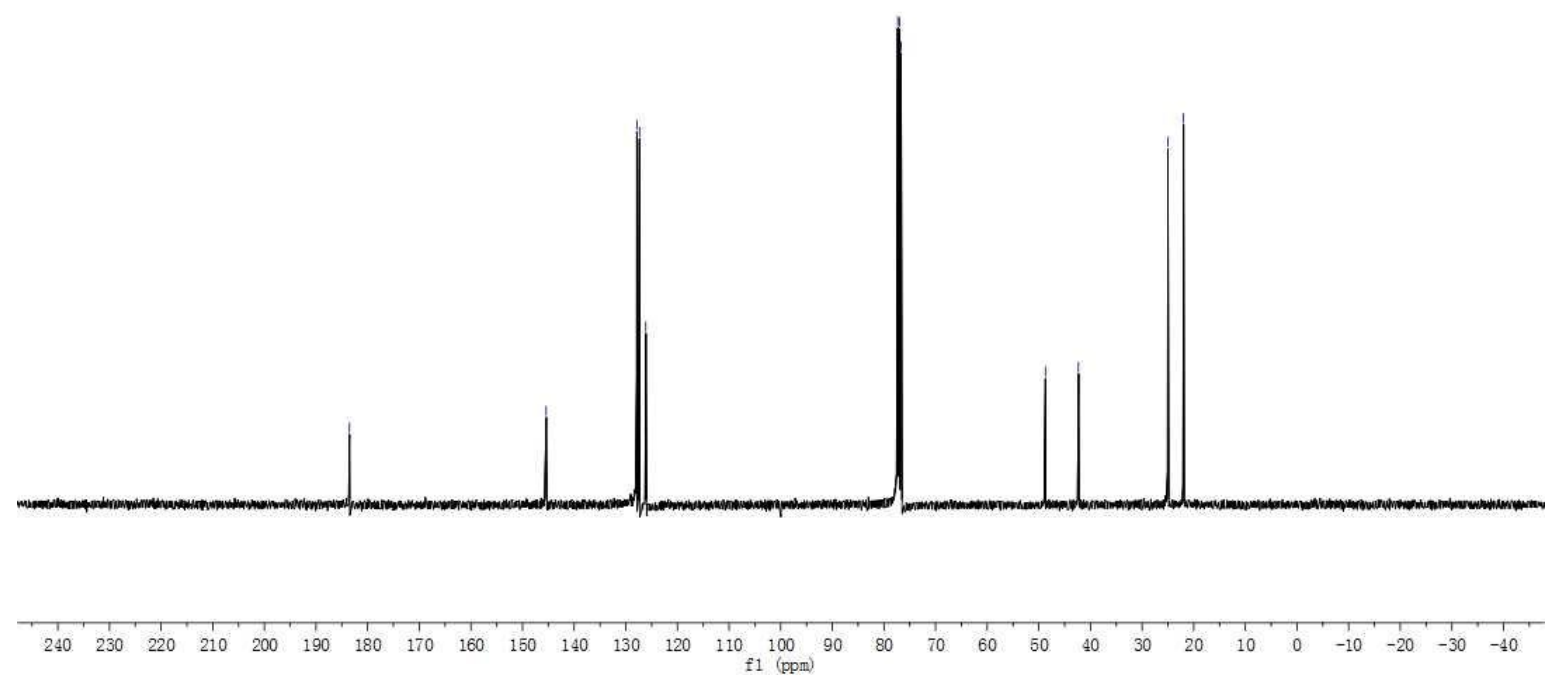

S120 


\section{3-(4-methoxyphenyl)-2,2,3-trimethyl-1-phenylbutyl-3,5-dinitrobenzoate (9)}
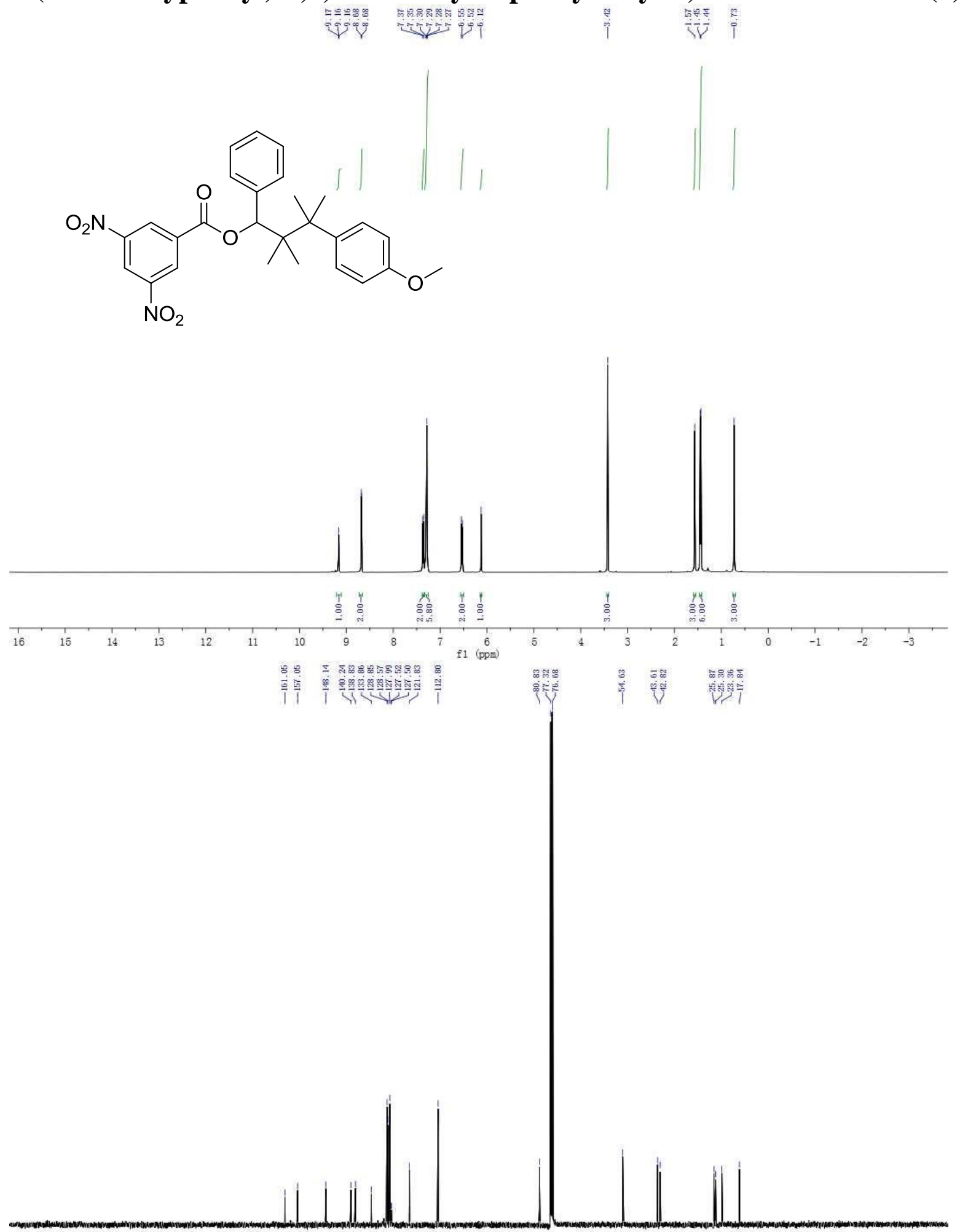

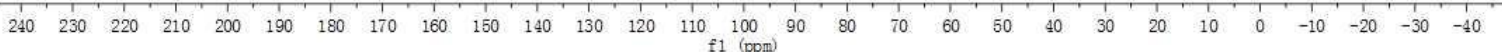




\section{DFT Calculation}

All calculations were performed with the Gaussian 09 suite. ${ }^{1}$ Density functional theory (DFT) with UB3LYP functional was used to perform geometry optimization of intermediates (A-2a, A-2c, B), and B3LYP for products $(\mathbf{C}-\mathbf{M e}, \mathbf{C}-\mathbf{t} \mathbf{B u})$ along various possible reaction paths. The basis set of 6-311G(d,p) was adopted. The performance of the selected calculation level was validated by the good agreements between the B3LYP/6-311G(d,p) frequency, NMR, and time dependent DFT (TD-DFT) calculations and the experimental IR, H-NMR and the UV-vis spectra of the reactants and product, respectively.

1) The cleavage of radical intermediate $\mathbf{A}$ to $\mathbf{C}$ and $\mathbf{B}$<smiles>[R20]C(=O)c1c(C)nc(C)c(C(=O)O)c1Cc1ccccc1</smiles>

A<smiles>[R]OC(=O)c1cc(C(=O)O[R20])c(C)nc1C</smiles>

C<smiles>Cc1ccccc1</smiles>

B<smiles>COC(=O)C1=C(C)N=C(C)C(Cc2ccccc2)C1C(=O)OC</smiles>

A-2a<smiles>CCCCOC(=O)C1=C(C)NC(C)=C(C(=O)OC(C)(C)C)C1Cc1ccccc1</smiles>

A-2c<smiles>COC(=O)c1cc(C(=O)OC)c(C)nc1C</smiles>

C-Me<smiles>CCC(C)OC(=O)c1cc(C(=O)OCC(C)C)c(C)nc1C</smiles>

C-tBu<smiles>Cc1ccccc1</smiles>

B

\begin{tabular}{|l|l|}
\hline $\mathrm{R}$ & $\triangle \mathrm{G}_{+\mathrm{ZPE}}$ \\
\hline $\mathrm{Me}$ & $-23.0999 \mathrm{Kcal} / \mathrm{mol}$ \\
\hline$t$-Bu & $-25.7593 \mathrm{Kcal} / \mathrm{mol}$ \\
\hline
\end{tabular}

The activation energy to the transition state of cleavage step is about $6.0 \mathrm{Kcal} / \mathrm{mol}$, suggesting a very fast process for both $\mathbf{2 a}$ and $\mathbf{2 c}$.

\section{Reference}

1. Frisch, M. J. et al. Gaussian 09 Revision B.01.Gaussian Inc. Wallingford CT 2009. 


\section{Coordinates for calculated geometries:}

1. A-2a<smiles>COC(=O)C1=C(C)N=C(C)C(Cc2ccccc2)C1C(=O)OC</smiles>

A-2a

\section{$\mathrm{O}$}

$\mathrm{O}$

$\mathrm{O}$

$\mathrm{O}$

$\mathrm{N}$

C

C

C

$\mathrm{H}$

$\mathrm{C}$

C

C

$\mathrm{H}$

$\mathrm{H}$

$\mathrm{H}$

$\mathrm{C}$

$\mathrm{H}$

$\mathrm{H}$

$\mathrm{H}$

$\mathrm{C}$

C

C

$\mathrm{H}$

$\mathrm{H}$

$\mathrm{C}$

C

C

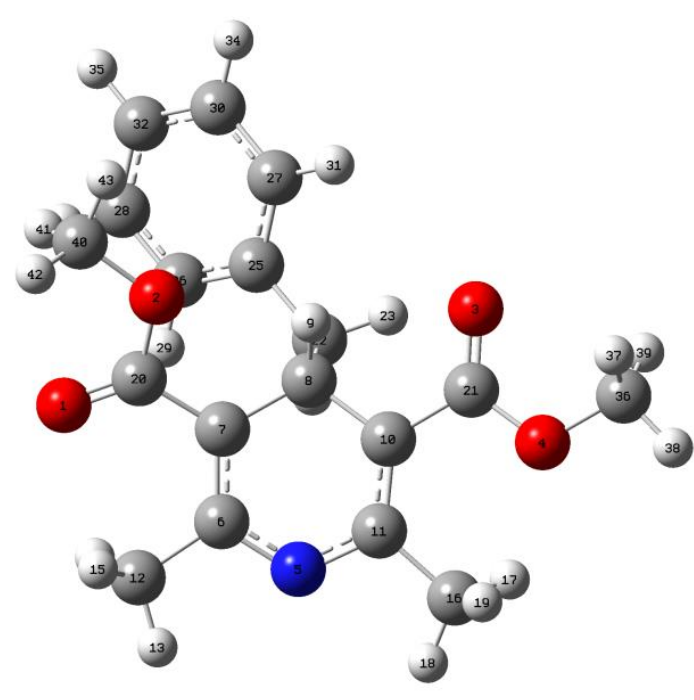

$$
\begin{array}{rrr}
2.10573 & 2.55236 & 0.71108 \\
1.73361 & 0.56388 & 1.69867
\end{array}
$$

$\begin{array}{lll}-1.99942 & -2.28641 & 0.76849\end{array}$

$\begin{array}{lll}-3.96812 & -1.32282 & 0.27193\end{array}$

$\begin{array}{lll}-1.92079 & 2.23485 & -0.74512\end{array}$

$\begin{array}{lll}-0.65122 & 2.40414 & -0.30697\end{array}$

$\begin{array}{lll}0.07385 & 1.35504 & 0.24955\end{array}$

$\begin{array}{lll}-0.49032 & -0.02606 & 0.18712\end{array}$

$\begin{array}{lll}-0.21334 & -0.59990 & 1.07134\end{array}$

$\begin{array}{lll}-1.97822 & -0.03753 & 0.01222\end{array}$

$\begin{array}{lll}-2.60291 & 1.08044 & -0.52202\end{array}$

$\begin{array}{lll}-0.13272 & 3.8134 & -0.44984\end{array}$

$\begin{array}{lll}-0.91722 & 4.42203 & -0.89673\end{array}$

$\begin{array}{lll}0.76661 & 3.84617 & -1.0667\end{array}$

$\begin{array}{lll}0.15262 & 4.23213 & 0.51778\end{array}$

$\begin{array}{lll}-4.06137 & 1.16975 & -0.90319\end{array}$

$\begin{array}{lll}-4.36655 & 0.34382 & -1.5464\end{array}$

$\begin{array}{lll}-4.21762 & 2.11851 & -1.41376\end{array}$

$\begin{array}{lll}-4.6997 & 1.12935 & -0.01762\end{array}$

$\begin{array}{lll}1.39845 & 1.58079 & 0.87241\end{array}$

$\begin{array}{lll}-2.62154 & -1.31656 & 0.38042\end{array}$

$\begin{array}{llll}0.13799 & -0.82846 & -1.05715\end{array}$

$-0.43714 \quad-1.75125 \quad-1.13313$

$-0.04781 \quad-0.23441 \quad-1.95501$

$\begin{array}{lll}1.60085 & -1.1687 & -0.94055\end{array}$

$\begin{array}{llll}2.57902 & -0.40870 & -1.58879\end{array}$

$\begin{array}{llll}2.00543 & -2.27961 & -0.18939\end{array}$ 


$\begin{array}{llcc}\mathrm{C} & 3.92828 & -0.74526 & -1.48835 \\ \mathrm{H} & 2.2835 & 0.45064 & -2.18191 \\ \mathrm{C} & 3.35057 & -2.61875 & -0.08769 \\ \mathrm{H} & 1.25442 & -2.87889 & 0.31512 \\ \mathrm{C} & 4.31806 & -1.85112 & -0.73692 \\ \mathrm{H} & 4.67213 & -0.14592 & -2.00145 \\ \mathrm{H} & 3.6455 & -3.48640 & 0.49240 \\ \mathrm{H} & 5.36641 & -2.11786 & -0.66233 \\ \mathrm{C} & -4.61357 & -2.55231 & 0.64788 \\ \mathrm{H} & -4.40874 & -2.78744 & 1.69332 \\ \mathrm{H} & -5.67641 & -2.37913 & 0.49426 \\ \mathrm{H} & -4.26265 & -3.37576 & 0.02428 \\ \mathrm{C} & 3.02202 & 0.67211 & 2.32626 \\ \mathrm{H} & 3.80734 & 0.70692 & 1.57041 \\ \mathrm{H} & 3.07299 & 1.57218 & 2.94109 \\ \mathrm{H} & 3.12091 & -0.22045 & 2.93944\end{array}$

\section{A-2c}<smiles>CCCCOC(=O)C1=C(C)N=C(C)C(Cc2ccccc2)C1COC(C)(C)C</smiles>

A-2c

C

C

$\mathrm{N}$

C

C

$\mathrm{C}$

C

C

C

C

C

C

C

C

C

C

$\mathrm{O}$

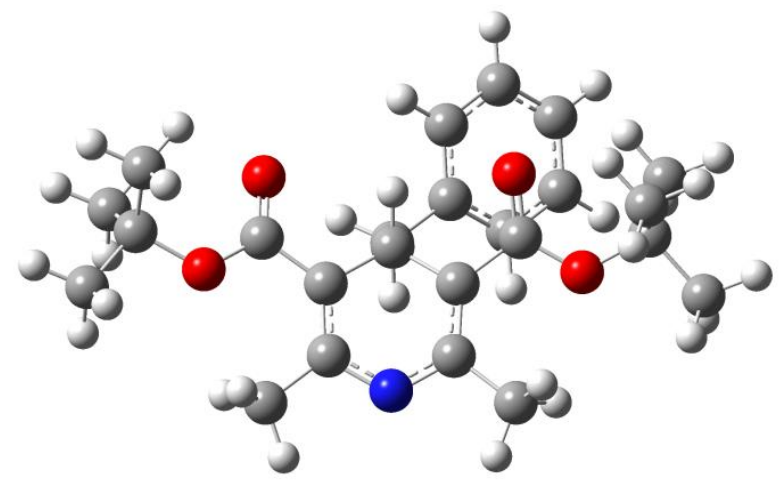
0.71343
$-0.96065$
$-0.16952$
$\begin{array}{lll}0.49832 & -2.0901 & -0.95084\end{array}$
$-0.72678 \quad-2.37499 \quad-1.45748$
$\begin{array}{llll}-1.83419 & -1.68289 & -1.08188\end{array}$
$-1.74117 \quad-0.53584 \quad-0.30642$
$\begin{array}{lll}-0.38456 & 0.04541 & -0.05274\end{array}$
$\begin{array}{llll}1.54160 & -3.12842 & -1.29134\end{array}$
$-3.11657 \quad-2.29844 \quad-1.58836$
$\begin{array}{lll}-0.17489 & 1.22068 & -1.12505\end{array}$
$\begin{array}{lll}1.05665 & 2.06932 & -0.94031\end{array}$
$\begin{array}{lll}2.16631 & 1.92353 & -1.77843\end{array}$
$\begin{array}{llll}3.28422 & 2.74399 & -1.63411\end{array}$
$\begin{array}{lll}3.31029 & 3.71933 & -0.64075\end{array}$
$\begin{array}{lll}2.21190 & 3.86978 & 0.20563\end{array}$
$\begin{array}{lll}1.09614 & 3.05337 & 0.05522\end{array}$
$\begin{array}{lll}-2.86410 & 0.25880 & 0.25058\end{array}$
$\begin{array}{lll}-2.68599 & 1.3391 & 0.78261\end{array}$ 


\begin{tabular}{|c|c|c|c|}
\hline $\mathrm{O}$ & -4.06484 & -0.33848 & 0.13682 \\
\hline $\mathrm{C}$ & -5.29669 & 0.28053 & 0.68006 \\
\hline $\mathrm{C}$ & -5.17604 & 0.43459 & 2.19846 \\
\hline $\mathrm{C}$ & -5.57049 & 1.61313 & -0.02198 \\
\hline $\mathrm{C}$ & -6.36982 & -0.74915 & 0.32551 \\
\hline $\mathrm{C}$ & 1.92336 & -0.67713 & 0.65017 \\
\hline $\mathrm{O}$ & 1.87744 & 0.05521 & 1.61759 \\
\hline $\mathrm{O}$ & 3.02613 & -1.32616 & 0.23641 \\
\hline $\mathrm{C}$ & 4.30021 & -1.25258 & 0.99223 \\
\hline $\mathrm{C}$ & 4.81165 & 0.18896 & 1.03099 \\
\hline $\mathrm{C}$ & 4.10467 & -1.83868 & 2.39293 \\
\hline $\mathrm{C}$ & 5.23096 & -2.13421 & 0.15907 \\
\hline $\mathrm{H}$ & -0.34744 & 0.52495 & 0.92538 \\
\hline $\mathrm{H}$ & 2.39398 & -2.6872 & -1.80894 \\
\hline $\mathrm{H}$ & 1.07349 & -3.88233 & -1.92217 \\
\hline $\mathrm{H}$ & 1.93470 & -3.60597 & -0.39131 \\
\hline $\mathrm{H}$ & -2.85650 & -3.0611 & -2.32093 \\
\hline $\mathrm{H}$ & -3.67206 & -2.76536 & -0.7717 \\
\hline $\mathrm{H}$ & -3.77895 & -1.55787 & -2.0361 \\
\hline $\mathrm{H}$ & -0.17182 & 0.7611 & -2.11637 \\
\hline $\mathrm{H}$ & -1.06068 & 1.85148 & -1.04381 \\
\hline $\mathrm{H}$ & 2.14910 & 1.17021 & -2.5596 \\
\hline $\mathrm{H}$ & 4.13034 & 2.62519 & -2.30232 \\
\hline $\mathrm{H}$ & 4.17743 & 4.36049 & -0.52808 \\
\hline $\mathrm{H}$ & 2.22375 & 4.62814 & 0.98063 \\
\hline $\mathrm{H}$ & 0.24214 & 3.17580 & 0.7129 \\
\hline $\mathrm{H}$ & -6.13695 & 0.75971 & 2.60640 \\
\hline $\mathrm{H}$ & -4.92026 & -0.52418 & 2.65657 \\
\hline $\mathrm{H}$ & -4.41634 & 1.16743 & 2.46341 \\
\hline $\mathrm{H}$ & -6.54805 & 1.9909 & 0.28986 \\
\hline $\mathrm{H}$ & -5.59177 & 1.4745 & -1.10601 \\
\hline $\mathrm{H}$ & -4.81332 & 2.3543 & 0.2261 \\
\hline $\mathrm{H}$ & -6.14749 & -1.71239 & 0.78963 \\
\hline $\mathrm{H}$ & -7.34474 & -0.40950 & 0.68310 \\
\hline $\mathrm{H}$ & -6.42813 & -0.89146 & -0.75567 \\
\hline $\mathrm{H}$ & 5.81711 & 0.20081 & 1.46112 \\
\hline $\mathrm{H}$ & 4.86545 & 0.60084 & 0.02111 \\
\hline $\mathrm{H}$ & 4.16329 & 0.82485 & 1.63007 \\
\hline $\mathrm{H}$ & 5.07112 & -1.89029 & 2.90159 \\
\hline $\mathrm{H}$ & 3.70091 & -2.85261 & 2.33024 \\
\hline $\mathrm{H}$ & 3.43053 & -1.22402 & 2.98692 \\
\hline $\mathrm{H}$ & 5.33145 & -1.73753 & -0.85355 \\
\hline $\mathrm{H}$ & 6.22222 & -2.16711 & 0.61740 \\
\hline $\mathrm{H}$ & 4.84582 & -3.15406 & 0.09430 \\
\hline
\end{tabular}


<smiles>COC(=O)c1cc(C(=O)OC)c(C)nc1C</smiles>

O

$\mathrm{O}$

$\mathrm{O}$

$\mathrm{O}$

$\mathrm{N}$

C

C

C

$\mathrm{H}$

C

C

C

$\mathrm{H}$

$\mathrm{H}$

$\mathrm{H}$

C

$\mathrm{H}$

$\mathrm{H}$

$\mathrm{H}$

C

C

C

$\mathrm{H}$

$\mathrm{H}$

$\mathrm{H}$

C

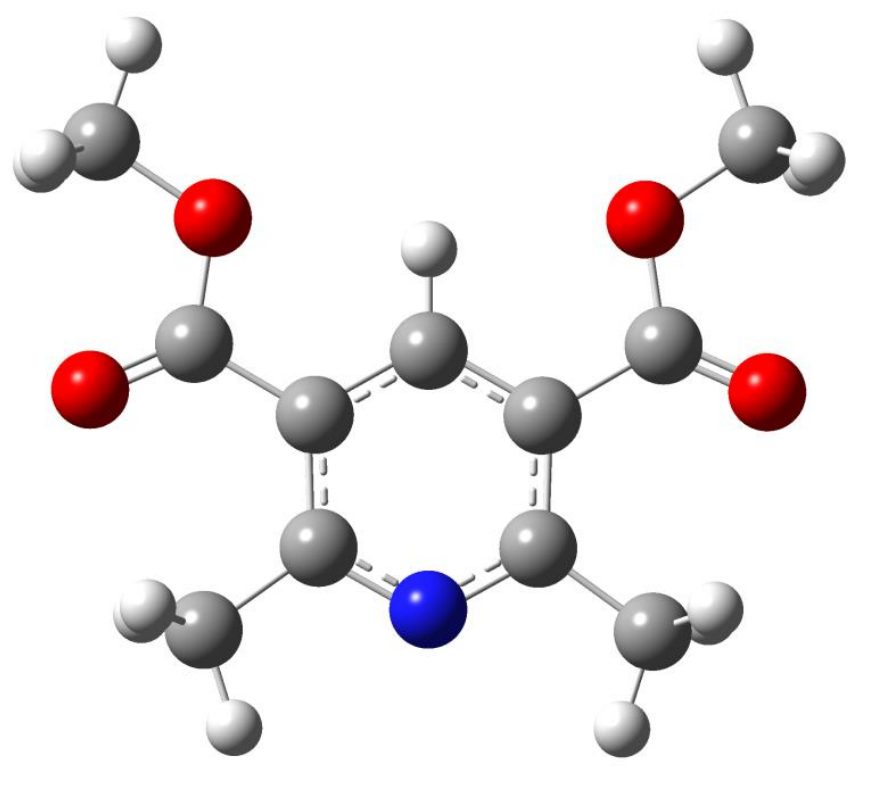

$\begin{array}{rcc}3.60333 & -0.01117 & 0.00038 \\ 2.30076 & 1.81961 & -0.00017 \\ -3.60335 & -0.01117 & -0.00061 \\ -2.30077 & 1.8196 & 0.00030 \\ 0.00001 & -2.33553 & 0.00003 \\ 1.16899 & -1.68255 & -0.00006 \\ 1.21201 & -0.26958 & 0.00005 \\ -0.00001 & 0.41674 & 0.00010 \\ -0.00002 & 1.49594 & 0.00010 \\ -1.21203 & -0.26960 & 0.00009 \\ -1.16899 & -1.68257 & 0.00015 \\ 2.38982 & -2.56081 & -0.00026 \\ 3.01873 & -2.36385 & 0.87050 \\ 3.01843 & -2.36384 & -0.87121 \\ 2.06458 & -3.59973 & -0.00023 \\ -2.38979 & -2.56086 & 0.00026 \\ -2.06453 & -3.59977 & 0.00171 \\ -3.01768 & -2.36503 & -0.87153 \\ -3.01943 & -2.36280 & 0.87017 \\ 2.49882 & 0.48101 & 0.00011 \\ -2.49884 & 0.48100 & -0.0001 \\ -3.49319 & 2.62303 & 0.00012 \\ -4.09307 & 2.41746 & 0.88788 \\ -3.14752 & 3.65429 & 0.00072 \\ -4.09228 & 2.41823 & -0.88835 \\ 3.49321 & 2.62300 & -0.00021\end{array}$




$\begin{array}{lrrr}\mathrm{H} & 4.09309 & 2.41715 & -0.88790 \\ \mathrm{H} & 3.14756 & 3.65427 & -0.00110 \\ \mathrm{H} & 4.09228 & 2.41843 & 0.88833\end{array}$

\section{C-tBu}<smiles>CCCCOC(=O)c1cc(C(=O)OCC(C)C)c(C)nc1C</smiles>

C-tBu
C

C

$\mathrm{N}$

C

C

C

C

C

C

C

$\mathrm{O}$

O

$\mathrm{O}$

C

C

C

C

$\mathrm{O}$

C

C

C

C

$\mathrm{H}$

$\mathrm{H}$

$\mathrm{H}$

$\mathrm{H}$

$\mathrm{H}$

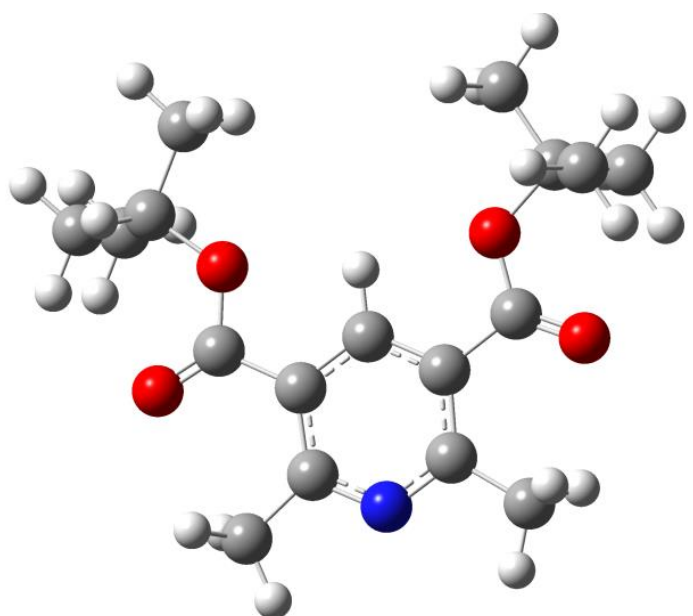

$\begin{array}{lll}-1.21368 & 1.20042 & 0.00025\end{array}$

$\begin{array}{lll}-1.16967 & 2.61364 & -0.00116\end{array}$

$\begin{array}{lll}0.00000 & 3.26570 & -0.00180\end{array}$

$\begin{array}{lll}1.16967 & 2.61364 & -0.00079\end{array}$

$\begin{array}{lll}1.21368 & 1.20042 & 0.00060\end{array}$

$\begin{array}{lll}0.00000 & 0.51656 & 0.00091\end{array}$

$\begin{array}{llll}2.3869 & 3.49793 & -0.00147\end{array}$

$\begin{array}{lll}-2.38689 & 3.49793 & -0.00217\end{array}$

$\begin{array}{lll}2.50596 & 0.44802 & 0.00202\end{array}$

$\begin{array}{lll}-2.50596 & 0.44803 & 0.00122\end{array}$

$\begin{array}{lll}-3.59995 & 0.96816 & 0.00344\end{array}$

$\begin{array}{lll}3.59995 & 0.96816 & 0.00653\end{array}$

$\begin{array}{lll}-2.29767 & -0.88337 & -0.00089\end{array}$

$\begin{array}{lll}-3.41647 & -1.85216 & -0.00015\end{array}$

$-2.68880 \quad-3.19655 \quad-0.00301$

$\begin{array}{lll}-4.24943 & -1.68823 & 1.27378\end{array}$

$\begin{array}{lll}-4.25366 & -1.68510 & -1.2709\end{array}$

$\begin{array}{lll}2.29767 & -0.88337 & -0.00205\end{array}$

$\begin{array}{llll}3.41646 & -1.85216 & -0.00097\end{array}$

$\begin{array}{lll}2.68880 & -3.19655 & -0.00628\end{array}$

$\begin{array}{llll}4.25527 & -1.68369 & -1.27046\end{array}$

$\begin{array}{lll}4.24780 & -1.68966 & 1.27421\end{array}$

$\begin{array}{lll}0.00000 & -0.56213 & 0.00174\end{array}$

$\begin{array}{llll}2.05524 & 4.53494 & -0.00462\end{array}$

$\begin{array}{lll}3.01533 & 3.30678 & 0.87079\end{array}$

$\begin{array}{lll}3.01804 & 3.30223 & -0.87075\end{array}$

$\begin{array}{lll}-3.01740 & 3.30269 & -0.87200\end{array}$ 


$\begin{array}{lccc}\mathrm{H} & -3.01595 & 3.30633 & 0.86953 \\ \mathrm{H} & -2.05523 & 4.53495 & -0.00455 \\ \mathrm{H} & -3.41345 & -4.01425 & -0.00304 \\ \mathrm{H} & -2.05910 & -3.29104 & -0.89036 \\ \mathrm{H} & -2.05651 & -3.29344 & 0.88224 \\ \mathrm{H} & -4.98622 & -2.49405 & 1.33050 \\ \mathrm{H} & -4.77294 & -0.7341 & 1.28506 \\ \mathrm{H} & -3.60821 & -1.75263 & 2.15660 \\ \mathrm{H} & -4.77721 & -0.73095 & -1.27807 \\ \mathrm{H} & -3.61538 & -1.74730 & -2.15600 \\ \mathrm{H} & -4.99062 & -2.49080 & -1.32715 \\ \mathrm{H} & 3.41345 & -4.01425 & -0.00647 \\ \mathrm{H} & 2.05550 & -3.29451 & 0.87813 \\ \mathrm{H} & 2.06012 & -3.28995 & -0.89446 \\ \mathrm{H} & 4.99221 & -2.48940 & -1.32674 \\ \mathrm{H} & 4.77893 & -0.72958 & -1.27585 \\ \mathrm{H} & 3.61810 & -1.74476 & -2.15644 \\ \mathrm{H} & 4.77119 & -0.73548 & 1.28727 \\ \mathrm{H} & 3.60548 & -1.75517 & 2.15614 \\ \mathrm{H} & 4.98461 & -2.49547 & 1.33089\end{array}$

5 Benzyl radical

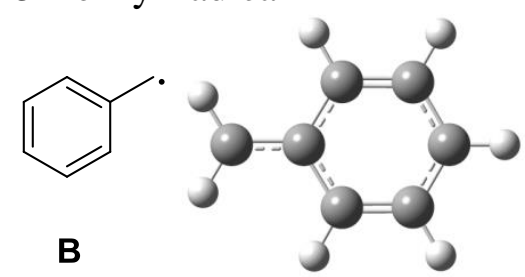

$\begin{array}{lrrr}\mathrm{C} & -1.13151 & -1.20981 & -0.00001 \\ \mathrm{C} & -1.83630 & 0.00000 & 0.00001 \\ \mathrm{C} & -1.13159 & 1.20979 & 0.00000 \\ \mathrm{C} & 0.25120 & 1.21647 & -0.00003 \\ \mathrm{C} & 0.99417 & 0.00001 & 0.00005 \\ \mathrm{C} & 0.25123 & -1.21649 & 0.00002 \\ \mathrm{C} & 2.39807 & 0.00005 & -0.00006 \\ \mathrm{H} & -1.67392 & -2.14900 & 0.00001 \\ \mathrm{H} & -2.92000 & -0.00008 & 0.00000 \\ \mathrm{H} & -1.67396 & 2.14901 & -0.00003 \\ \mathrm{H} & 0.79081 & 2.15761 & -0.00001 \\ \mathrm{H} & 0.79091 & -2.15759 & -0.00001 \\ \mathrm{H} & 2.95734 & 0.92717 & 0.00029 \\ \mathrm{H} & 2.95721 & -0.92719 & -0.00010\end{array}$


2) The direct coupling between the anionic radical and benzylic radical

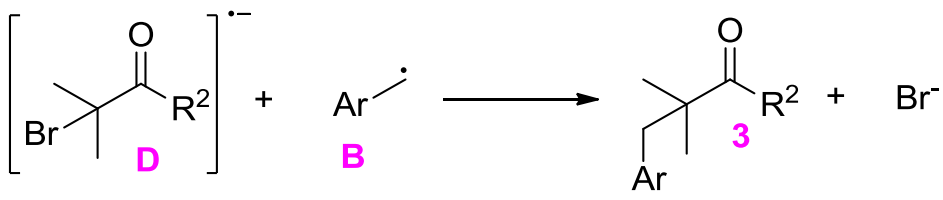

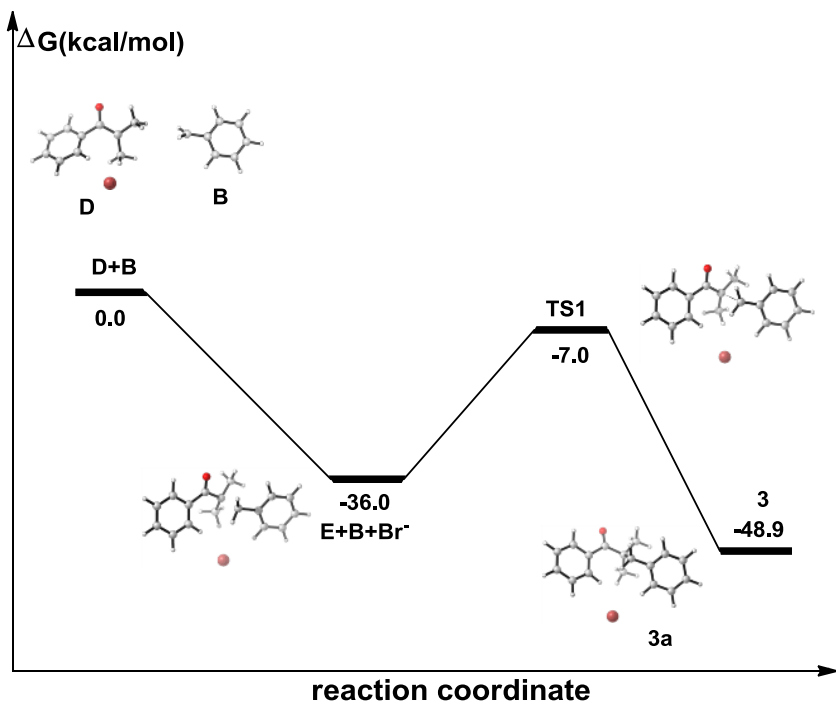

The total Gibbs free energy of $\mathbf{D}+\mathbf{B}$ is higher than that of TS-1, which might help the direct coupling of $\mathbf{D}$ and $\mathbf{B}$ through TS-1 instead of mesolysis to $\mathbf{E}+\mathbf{B}+\mathrm{Br}^{-}$at first.

Coordinates for calculated geometries:

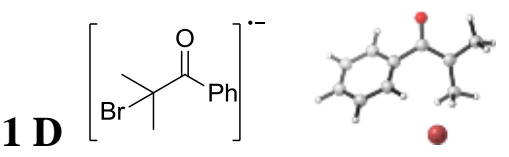

$\begin{array}{lrrr}\mathrm{C} & 0.76879900 & -0.98339900 & -0.08832500 \\ \mathrm{C} & 1.76526800 & 0.00101500 & -0.05979000 \\ \mathrm{C} & 3.10744800 & -0.39161200 & 0.04086900 \\ \mathrm{C} & 3.44882700 & -1.73447300 & 0.14958500 \\ \mathrm{C} & 2.45039700 & -2.71060800 & 0.13193800 \\ \mathrm{C} & 1.11684900 & -2.33102600 & 0.00329200 \\ \mathrm{H} & -0.28184400 & -0.73012000 & -0.20624200 \\ \mathrm{H} & 3.87014000 & 0.37800400 & 0.01836100 \\ \mathrm{H} & 4.49164600 & -2.02306000 & 0.23994400 \\ \mathrm{H} & 2.71404400 & -3.76091200 & 0.21037400 \\ \mathrm{H} & 0.32909700 & -3.07551600 & -0.02955800 \\ \mathrm{C} & 1.47288300 & 1.46879800 & -0.23889800 \\ \mathrm{C} & 0.28432700 & 2.06820100 & 0.31846700 \\ \mathrm{O} & 2.30424600 & 2.16223100 & -0.86073400 \\ \mathrm{Br} & -3.11142200 & -0.70888100 & -0.17535600 \\ \mathrm{C} & -0.60638400 & 1.41661700 & 1.31013700 \\ \mathrm{H} & -0.09873100 & 0.65487200 & 1.90422400 \\ \mathrm{H} & -1.04355300 & 2.16350500 & 1.98211800 \\ \mathrm{H} & -1.46519200 & 0.90085100 & 0.81751300\end{array}$




$\begin{array}{lrrr}\mathrm{C} & -0.06501100 & 3.45704200 & -0.11313100 \\ \mathrm{H} & -0.04626600 & 4.15001600 & 0.73951300 \\ \mathrm{H} & 0.62638000 & 3.81687200 & -0.87341800 \\ \mathrm{H} & -1.09035200 & 3.47513400 & -0.50436000\end{array}$

2 B<smiles>CC1=C(C)C(C)C(Cc2ccccc2)C(C)C1C</smiles>

C

$\begin{array}{lll}0.00000000 & 1.20362300 & 0.24925100 \\ 0.00000000 & 0.00000000 & 0.97001100\end{array}$

$\mathrm{C}$

$0.00000000-0.00000000$

0.97001100

$\mathrm{C}$

$\begin{array}{lll}0.00000000 & -1.20362300 & 0.24925100\end{array}$

$\mathrm{C}$

$-0.00999700$

$-1.20332200$

$-1.14347000$

C

0.00000000

0.00000000

$-1.84491100$

$\mathrm{C}$

0.00999700

1.20332200

$-1.14347000$

$\mathrm{H}$

0.00672300

2.14423300

0.78936500

$\mathrm{H}$

$-0.00672300$

$-2.14423300$

0.78936500

$\mathrm{H}$

$-0.02174100$

$-2.14563300$

$-1.68053700$

$\mathrm{H}$

0.00000000

0.00000000

$-2.92925000$

$\mathrm{H}$

0.02174100

2.14563300

$-1.68053700$

C

0.00000000

0.00000000

2.44685700

$\mathrm{H}$

0.92499200

$-0.36653100$

3.00523700

$\mathrm{H}$

$-0.92499200$

0.36653100

3.00523700

3 Intermediate $\mathbf{E}+\mathbf{B}+\mathrm{Br}^{-}$<smiles>CC(Br)C(=O)c1ccccc1</smiles><smiles>CC(C)C(C)(C)C(C)(C)C(C)(C)C(C)(C)C(C)(C)Cc1ccccc1</smiles>

C

C

C

C

C

C

$\mathrm{H}$

$\mathrm{H}$

$\mathrm{H}$

$\mathrm{H}$

$\mathrm{H}$

C

C

$\mathrm{O}$

$\mathrm{Br}$

C

$\mathrm{H}$

$\mathrm{H}$
$-2.57168200$

$-2.59063700$

$-3.80284900$

$-4.96873700$

$-4.94230400$

$-3.74344200$

$-1.66345100$

$-3.79835800$

$-5.89649900$

$-5.85069700$

$-3.69540700$

$-1.36876000$

$-0.06318700$

$-1.40985200$

0.16899800

$-0.21619600$

$-1.22457000$

0.47591900
0.38162900

$-1.01489300$

$-0.15683300$

$-1.69437600$

$-0.02857200$

$-1.00296800$

$-0.24096700$

0.38874800

$-0.54187800$

1.07135900

0.96519300

$-0.65769600$

$-0.47512100$

$-2.77544100$

$-0.03920000$

$-1.54608600$

$-0.17418600$

0.93344600

$-0.69183500$

2.14963400

$-0.89561300$

$-1.87398100$

$-0.57717500$

$-1.31797400$

0.23102000

$-3.04416400$

0.83355400

3.09474800

$-0.12468800$

$-0.11545000$

0.11575600

$-0.02934700$

1.79920500

$-0.22269100$

2.63884300 
$\mathrm{H}$

C

$\mathrm{H}$

$\mathrm{H}$

$\mathrm{H}$

C

C

C

C

C

C

$\mathrm{H}$

$\mathrm{H}$

$\mathrm{H}$

$\mathrm{H}$

$\mathrm{H}$

C

$\mathrm{H}$

$\mathrm{H}$
0.01155700

0.74487600

0.28370100

0.85324800

1.73772800

3.27272500

2.40279700

2.98429100

4.36617100

5.20628700

4.65500300

2.85499800

2.33664400

4.78315100

6.27961700

5.29829800

0.88627500

0.43932000

0.63491400
0.83740700

$-2.45887600$

$-2.85727800$

$-3.28221800$

$-2.08391200$

$-1.80275300$

$-0.71704000$

0.52760600

0.65445200

$-0.44402700$

$-1.67311400$

$-2.76677700$

1.39103100

1.62114800

$-0.34220300$

$-2.53271000$

$-0.87482300$

0.05977000

$-1.68292200$
1.31317200

1.45789100

2.36987800

0.75153000

1.71450300

$-0.81312300$

$-0.58622500$

$-0.27331600$

$-0.12391400$

$-0.30628000$

$-0.66612000$

$-1.08808300$

$-0.12880200$

0.13978800

$-0.17850600$

$-0.82854100$

$-0.65914300$

$-1.00005200$

$-1.34974800$

\section{TS-1}

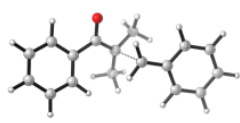

$\mathrm{C}$
$\mathrm{C}$
$\mathrm{C}$
$\mathrm{C}$
$\mathrm{C}$
$\mathrm{C}$
$\mathrm{H}$
$\mathrm{H}$
$\mathrm{H}$
$\mathrm{H}$
$\mathrm{H}$
$\mathrm{C}$
$\mathrm{C}$
$\mathrm{O}$
$\mathrm{Br}$
$\mathrm{C}$
$\mathrm{H}$
$\mathrm{H}$
$\mathrm{H}$
$\mathrm{C}$
$\mathrm{H}$

$-2.57725400$

0.38283600

$-0.16467200$

$-2.58841400$

$-1.00962500$

$-0.03142200$

$-3.80236800$

$-1.69038400$

$-0.24721800$

$-4.96598400$

$-1.00303900$

$-0.55204100$

$-4.94299800$

0.38944700

$-0.66756900$

$-3.74471700$

1.07374600

$-0.48265900$

$-1.67141000$

0.96660000

$-0.04866200$

$-3.79122300$

$-2.77185900$

$-0.18512900$

$-5.89236300$

$-1.54905800$

$-0.70179000$

$-5.85454100$

0.93451100

$-0.90775400$

$-3.70004400$

2.15295100

$-0.58726800$

$-1.38244600$

$-1.86449000$

0.26284000

$-0.22560600$

$-1.37495000$

0.98251500

$-1.39782900$

$-3.05611200$

$-0.13583000$

0.16964500

3.09285000

0.11404100

$-0.22081000$

$-0.11086300$

1.77982200

$-1.21412000$

0.12443600

2.17593700

0.48101700

$-0.18962000$

2.61471300

0.07266000

0.79353600

1.21088800

0.74430700

$-2.43733400$

1.42780800

0.35480500

$-2.89595000$

2.34850100 


$\begin{array}{lrrr}\mathrm{H} & 0.84424700 & -3.23945300 & 0.69366600 \\ \mathrm{H} & 1.72490200 & -2.01481200 & 1.65994500 \\ \mathrm{C} & 3.28535600 & -1.82326500 & -0.81535200 \\ \mathrm{C} & 2.40166200 & -0.71137500 & -0.62632700 \\ \mathrm{C} & 2.99388200 & 0.54787400 & -0.27143300 \\ \mathrm{C} & 4.36516200 & 0.65917600 & -0.10815800 \\ \mathrm{C} & 5.21189000 & -0.44467600 & -0.29509200 \\ \mathrm{C} & 4.65381800 & -1.68571700 & -0.65557400 \\ \mathrm{H} & 2.86481300 & -2.78793300 & -1.08381500 \\ \mathrm{H} & 2.34873500 & 1.40962800 & -0.13612400 \\ \mathrm{H} & 4.78585600 & 1.62639300 & 0.15510100 \\ \mathrm{H} & 6.28419100 & -0.34095200 & -0.17025800 \\ \mathrm{H} & 5.30223100 & -2.54410400 & -0.80523200 \\ \mathrm{C} & 1.01049200 & -0.85312600 & -0.74298600 \\ \mathrm{H} & 0.40085800 & 0.03794500 & -0.74882000 \\ \mathrm{H} & 0.60859600 & -1.77852700 & -1.14358800\end{array}$

\begin{tabular}{|c|c|c|c|}
\hline $\mathrm{C}$ & -3.62660400 & -1.65055300 & -0.39836500 \\
\hline $\mathrm{C}$ & -2.38606600 & -1.08037300 & -0.05597900 \\
\hline $\mathrm{C}$ & -2.23651400 & 0.30779500 & -0.17217000 \\
\hline $\mathrm{C}$ & -3.29610400 & 1.09955400 & -0.61530200 \\
\hline $\mathrm{C}$ & -4.51401000 & 0.52141500 & -0.95413800 \\
\hline $\mathrm{C}$ & -4.67689400 & -0.86265000 & -0.84489800 \\
\hline $\mathrm{H}$ & -3.73623600 & -2.72320900 & -0.30034700 \\
\hline $\mathrm{H}$ & -1.32448600 & 0.83809900 & 0.07495200 \\
\hline $\mathrm{H}$ & -3.13185200 & 2.16931800 & -0.68096500 \\
\hline $\mathrm{H}$ & -5.33537700 & 1.14076000 & -1.30121000 \\
\hline $\mathrm{H}$ & -5.62421900 & -1.32325200 & -1.10741000 \\
\hline $\mathrm{C}$ & -1.32337900 & -2.04241800 & 0.42456100 \\
\hline $\mathrm{C}$ & 0.14819400 & -1.62250200 & 0.69918400 \\
\hline $\mathrm{O}$ & -1.64084400 & -3.21009100 & 0.58935400 \\
\hline $\mathrm{Br}$ & -0.07432000 & 3.20732200 & 0.32932000 \\
\hline $\mathrm{C}$ & 0.21760700 & -0.58529500 & 1.84657500 \\
\hline $\mathrm{H}$ & -0.36700100 & -0.93360200 & 2.70446700 \\
\hline $\mathrm{H}$ & 1.25495900 & -0.48263500 & 2.17465100 \\
\hline $\mathrm{H}$ & -0.12542700 & 0.41171100 & 1.57093400 \\
\hline $\mathrm{C}$ & 0.91122200 & -2.88183500 & 1.14815000 \\
\hline $\mathrm{H}$ & 0.46695800 & -3.29596900 & 2.05545200 \\
\hline $\mathrm{H}$ & 0.88062100 & -3.66519100 & 0.38753000 \\
\hline $\mathrm{H}$ & 1.95343400 & -2.62996600 & 1.35237900 \\
\hline
\end{tabular}




$\begin{array}{lrrr}\mathrm{C} & 3.21410700 & -1.65120800 & -0.95367600 \\ \mathrm{C} & 2.23328700 & -0.71747900 & -0.59461500 \\ \mathrm{C} & 2.64226300 & 0.57069900 & -0.22267600 \\ \mathrm{C} & 3.99677300 & 0.89878600 & -0.19315100 \\ \mathrm{C} & 4.96459400 & -0.04291000 & -0.53840100 \\ \mathrm{C} & 4.56778600 & -1.32189600 & -0.92475400 \\ \mathrm{H} & 2.91239300 & -2.64672600 & -1.26550100 \\ \mathrm{H} & 1.89906200 & 1.32959600 & 0.02018700 \\ \mathrm{H} & 4.28800500 & 1.90415400 & 0.09241100 \\ \mathrm{H} & 6.01781800 & 0.21936300 & -0.51758500 \\ \mathrm{H} & 5.30993500 & -2.06144700 & -1.20966700 \\ \mathrm{C} & 0.76023900 & -1.07283900 & -0.63895000 \\ \mathrm{H} & 0.20640300 & -0.18740100 & -0.95007700 \\ \mathrm{H} & 0.60396400 & -1.83690000 & -1.40960200\end{array}$

